\title{
Angewandte
}

Supporting Information

(C) Wiley-VCH 2013

69451 Weinheim, Germany

Chiral Phosphoric Acid Directed Regioselective Acetalization of Carbohydrate-Derived 1,2-Diols**

Enoch Mensah, Nicole Camasso, Will Kaplan, and Pavel Nagorny*

ange_201304298_sm_miscellaneous_information.pdf 
I. General Methods..................................................

II. Regioselective Tetrahydropyranylations.............................2-19

Part I: Synthesis of Substrates 1d and 1e...........................2-5

Part II: General Procedure for Regioselective Tetrahydropyranylation..... 5-6

Part III: Regioselective Tetrahydropyranylation Reactions...............6-19

III. Regioselective Acetalization.....................................19-39

IV. Meso Diol Desymmetrization Studies/Reaction Mechanism.............39-41

V. NMR Spectra.............................................41-142

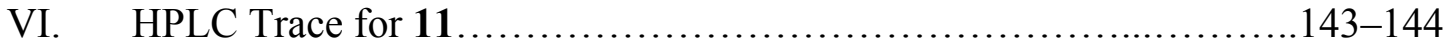




\section{General Methods}

Method and Reagents. All reactions were carried out under an atmosphere of nitrogen in oven dried glassware with a magnetic stirrer, unless otherwise noted. Air-sensitive reactions were cooled via external cooling baths: ice water $\left(0^{\circ} \mathrm{C}\right)$, dry ice-acetonitrile $(-$ $\left.50{ }^{\circ} \mathrm{C}\right)$, dry ice-acetone $\left(-78{ }^{\circ} \mathrm{C}\right)$, Neslab CB 80 immersion cooler $\left(10\right.$ to $\left.-60{ }^{\circ} \mathrm{C}\right)$ or Neslab cryocool immersion cooler CC-100II ( -20 to $\left.-100{ }^{\circ} \mathrm{C}\right)$. Heating was achieved by use of a silicone bath with heating controlled by electronic contact thermometer. Deionized water was used in the preparation of all aqueous solutions and for all aqueous extractions. Solvents used for extraction and column chromatography were ACS or HPLC grade. Reagents were purified prior to use following the guidelines of Perrin and Armarego. ${ }^{1}$ Tetrahydrofuran (THF) and dichloromethane $\left(\mathrm{CH}_{2} \mathrm{Cl}_{2}\right)$ were filtered through a column of activated alumina under nitrogen atmosphere (Innovative Technologies). Analytical thin-layer chromatography (TLC) was routinely used to monitor the progress of the reactions and performed using precoated glass plates with 230-400 mesh silica gel impregnated with a fluorescent indicator $(250 \mathrm{~nm})$. Visualization was achieved using UV light or ceric ammonium molybdate. Purification of the reactions mixtures was performed by flash column chromatography using SiliCycle Silia Flash P60 (230-400 mesh) silica gel. $4 \AA$ molecular sieves were also pre-activated before use.

Instrumentation. ${ }^{1} \mathrm{H}$ NMR spectra were recorded on Varian vnmrs $500(500 \mathrm{MHz})$, Varian INOVA $500(500 \mathrm{MHz})$ or Varian MR400 (400 MHz) spectrometers and chemical shifts $(\delta)$ are reported in parts per million $(\mathrm{ppm})$ with solvent resonance as the internal standard $\left(\mathrm{CDCl}_{3}\right.$ at $\delta 7.24, \mathrm{C}_{6} \mathrm{D}_{6}$ at $\delta 7.15$ and $\mathrm{CD}_{3} \mathrm{OD}$ at $\delta 4.78$ and 3.30). Data are reported as $(\mathrm{br}=$ broad, $\mathrm{s}=$ singlet, $\mathrm{d}=$ doublet, $\mathrm{t}=$ triplet, at $=$ apparent triplet, $\mathrm{q}=$ quartet, $\mathrm{qn}=$ quintet, sext $=$ sextet, $\mathrm{m}=$ multiplet; $\operatorname{coupling} \operatorname{constant}(\mathrm{s})$ in $\mathrm{Hz}$; integration). Proton-decoupled ${ }^{13} \mathrm{C}$ NMR spectra were recorded on Varian vnmrs 500 $(500 \mathrm{MHz})$ or Varian INOVA $500(500 \mathrm{MHz})$ spectrometers and chemical shifts $(\delta)$ are reported in ppm with solvent resonance as the internal standard $\left(\mathrm{CDCl}_{3}\right.$ at $\delta 77.0, \mathrm{C}_{6} \mathrm{D}_{6}$ at

1 Perrin, D. D.; Armarego, W. L. Purification of Laboratory Chemicals; Third Addition; Pergamon Press: Oxford, 1988. 
$\delta 127.7 \mathrm{CD}_{3} \mathrm{OD}$ at $\delta 49.0$ ). High resolution mass spectra (HRMS) were recorded on Micromass AutoSpec Ultima or VG (Micromass) 70-250-S Magnetic sector mass spectrometers at the University of Michigan mass spectrometry laboratory. Infrared (IR) spectra were recorded as thin films on $\mathrm{NaCl}$ plates on a Perkin Elmer Spectrum BX FTIR spectrometer. Transmittance (\%) peaks were reported in wavenumbers $\left(\mathrm{cm}^{-1}\right)$. Optical rotations were measured in $\mathrm{CH}_{2} \mathrm{Cl}_{2}$ on JASCO P-2000 polarimeter at $589 \mathrm{~nm}$ (D-line) and reported as follows: $[\alpha]_{24}{ }^{\mathrm{D}}\left(\mathrm{c} \mathrm{g} / 100 \mathrm{ml}, \mathrm{CH}_{2} \mathrm{Cl}_{2}\right)$.

Catalysts. Catalysts $(R)$ - and $(S)-\mathbf{2 a}, \mathbf{2} \mathbf{b}$ and $\mathbf{2 c}$ as well as diphenylphosphoric acid were purchased from Sigma Aldrich, dissolved in dichloromethane, washed with $4 \mathrm{~N} \mathrm{HCl}$, then dried with $\mathrm{Na}_{2} \mathrm{SO}_{4}$, concentrated and further dried under high vacuum for at least 48 h. Catalysts $(R)$ - and $(S)$-2d where prepared according to the known procedure reported by Yamamoto and coworkers (Jiao, P.; Nakashima, D.; Yamamoto, H. Angew. Chem. Int. $E d$. 2008, 47, 2411). Racemic 2d was obtained by mixing equimolar solutions of $(R)$ - and (S)-2d.

\section{Regioselective Tetrahydropyranylation.}

Part I : Synthesis of Substrates 1d and 1e.
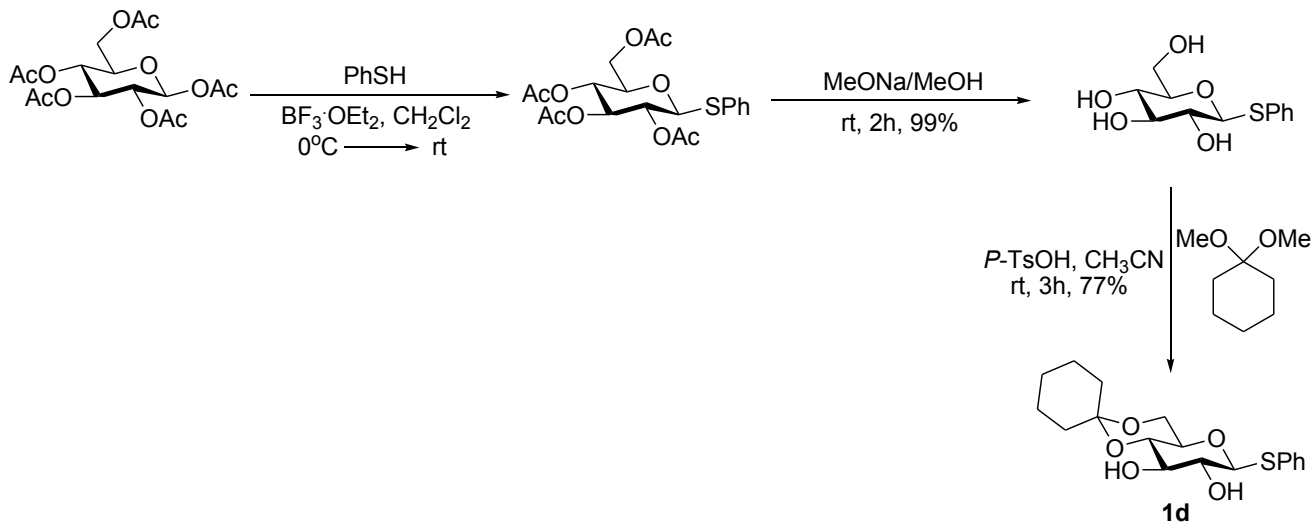

Synthesis of substrate 1d began using $\beta$-D-glucose pentaacetate $(10 \mathrm{~g}, 25.696 \mathrm{mmol}$,) as the starting material. This was converted to the corresponding thioglycoside in $86 \%$ yield, using the reported general protocol by Node et al. ${ }^{2}$ To this thioglycoside $(9.7 \mathrm{~g}, 22.098$ mmol, 1 equiv.) in a $250 \mathrm{~mL}$ round bottom flask was added methanol $(110 \mathrm{~mL})$, followed

2. Node et al. Carbohyd. Res. 2005, 340, $2360-2368$. 
by a small chip of sodium metal. The resulting pale yellow reaction mixture was stirred at room temperature for 2 hours. When the reaction was completed as monitored by TLC, the reaction was neutralized using few drops of ACS grade acetic acid, and concentrated in vacuo to form pale yellow oil as the crude tetraol. This was purified by flash column chromatography (10/1 Ethyl acetate / Methanol) to afford the pure tetraol as white solids (6 g, 99\%). To this tetraol (0.5 g, $1.836 \mathrm{mmol}, 1$ equiv.) in an oven dried and nitrogen flushed $50 \mathrm{~mL}$ round bottom flask, anhydrous acetonitrile $(15 \mathrm{~mL})$ was added. To the resulting white suspension, 1,1-dimethoxycyclohexane ( $0.4 \mathrm{~mL}, 2.754 \mathrm{mmol}, 1.5$ equiv.) was added followed by $p$-TsOH $(17.5 \mathrm{mg}, 0.092 \mathrm{mmol}, 5 \mathrm{~mol} \%)$. The resulting reaction mixture was stirred at room temperature for 3 hours. When the reaction was completed as indicated by TLC, the reaction mixture was quenched with triethylamine and concentrated in vacuo to form a pale yellow solid as the crude product. The crude product was purified by flash column chromatography (1/1 Hexanes/Ethyl acetate) to afford pure 1d as a pale yellow solid $(0.5 \mathrm{~g}, 77 \%)$.

${ }^{1}$ H NMR (500 MHz, $\left.\mathbf{C D C l}_{3}\right) \delta 7.54-7.49$ (m, 2H), $7.35-7.30(\mathrm{~m}, 3 \mathrm{H}), 4.58(\mathrm{~d}, J=9.7$ $\mathrm{Hz}, 1 \mathrm{H}), 3.95$ (dd, $J=10.8,5.4 \mathrm{~Hz}, 1 \mathrm{H}), 3.78$ (at, $J=10.5 \mathrm{~Hz}, 1 \mathrm{H}), 3.70$ (dd, $J=12.7$, $4.9 \mathrm{~Hz}, 1 \mathrm{H}), 3.55$ (at, $J=9.4 \mathrm{~Hz}, 1 \mathrm{H}), 3.44-3.39$ (m, 1H), 3.35 (td, $J=9.9,5.4 \mathrm{~Hz}, 1 \mathrm{H})$, $2.81(\mathrm{~s}, 1 \mathrm{H}), 2.72(\mathrm{~d}, J=1.8 \mathrm{~Hz}, 1 \mathrm{H}), 2.06-1.92(\mathrm{~m}, 1 \mathrm{H}), 1.91-1.79(\mathrm{~m}, 1 \mathrm{H}), 1.69-$ $1.53(\mathrm{~m}, 4 \mathrm{H}), 1.53-1.34(\mathrm{~m}, 4 \mathrm{H})$.

${ }^{13} \mathbf{C}$ NMR (125 MHz, $\left.\mathbf{C D C l}_{3}\right) \delta$ 132.9, 131.4, 129.0, 128.4, 99.9, 88.6, 77.3, 77.0, 76.8, $75.1,72.6,72.0,71.8,61.2,37.8,27.7,25.5,22.8,22.5$

IR (film, $\mathbf{~ c m}^{-1}$ ) 3429, 2936, 1649, 1261, 1077, 908. HRMS(ESI) Calc. for $\mathrm{C}_{18} \mathrm{H}_{24} \mathrm{O}_{5} \mathrm{SNa}$ $(\mathrm{M}+\mathrm{Na}): 375.1237$; found : 375.1234 .

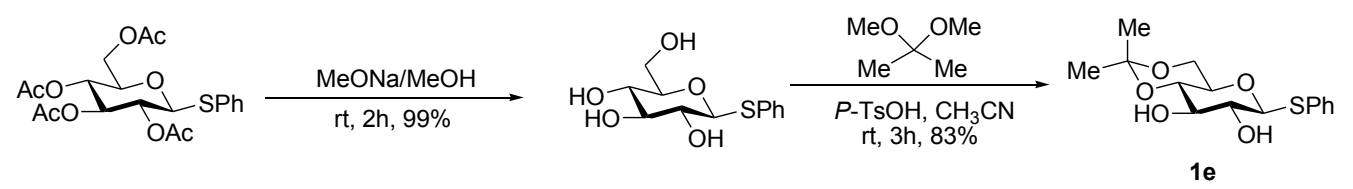

An oven dried $50 \mathrm{~mL}$ round bottom flask was charged with tetraacetate ${ }^{3}(1 \mathrm{~g}, 2.27 \mathrm{mmol}$, 1 equiv.), methanol $(17 \mathrm{~mL})$ and a small chip of sodium metal. The resulting pale yellow reaction mixture was stirred at room temperature for 2 hours. When the reaction was

3. Magnusson et al. Carbohydr. Res. 2000, 329, 49-55. 
completed as indicated by TLC, the reaction was neutralized using few drops of ACS grade acetic acid, and concentrated in vacuo to form a pale yellow oil as the crude tetraol. The crude tetraol was purified by flash column chromatography (10/1 Ethyl acetate / Methanol) to afford the pure tetraol as a white solid $(0.52 \mathrm{~g}, 84 \%)$. To this tetraol $(0.5 \mathrm{~g}$, $1.836 \mathrm{mmol}, 1$ equiv.) in an oven dried and nitrogen flushed $50 \mathrm{~mL}$ round bottom flask, anhydrous acetonitrile $(15 \mathrm{~mL})$ was added. To the resulting white suspension, 1,1dimethoxypropane $(0.25 \mathrm{~mL}, 2.754 \mathrm{mmol}, 1.2$ equiv.) was added, followed by $p$-TsOH (17.5 mg, $0.092 \mathrm{mmol}, 5 \mathrm{~mol} \%$ ). The resulting reaction mixture was stirred at room temperature for 3 hours. When the reaction was completed as indicated by TLC, the reaction mixture was quenched with triethylamine and concentrated in vacuo to form a yellow solid as the crude product, which was subsequently purified by flash column chromatography (1/2 Hexanes/Ethyl acetate) to afford pure 1e as a pale yellow foamy solid $(0.48 \mathrm{~g}, 83 \%)$.

${ }^{1}$ H NMR (500 MHz, $\left.\mathbf{C D C l}_{3}\right) \delta 7.55-7.47(\mathrm{~m}, 2 \mathrm{H}), 7.36-7.29(\mathrm{~m}, 3 \mathrm{H}), 4.59(\mathrm{~d}, J=9.7$ $\mathrm{Hz}, 1 \mathrm{H}$ ), 3.96 (dd, $J=10.8,5.3 \mathrm{~Hz}, 1 \mathrm{H}$ ), 3.78 (at, $J=10.6 \mathrm{~Hz}, 1 \mathrm{H}$ ), 3.69 (at, $J=8.8 \mathrm{~Hz}$, $1 \mathrm{H}$ ), 3.54 (at, $J=9.4 \mathrm{~Hz}, 1 \mathrm{H}$ ), 3.43 (at, $J=9.0 \mathrm{~Hz}, 1 \mathrm{H}$ ), 3.34 (td, $J=9.9,5.4 \mathrm{~Hz}, 1 \mathrm{H}$ ), $2.96(\mathrm{~s}, 1 \mathrm{H}), 2.80(\mathrm{~s}, 1 \mathrm{H}), 1.51(\mathrm{~d}, J=10.7 \mathrm{~Hz}, 3 \mathrm{H}), 1.43$ (s, 3H).

${ }^{13} \mathbf{C}$ NMR (125 MHz, $\left.\mathbf{C D C l}_{3}\right) \delta$ 132.8, 131.5, 129.1, 128.4, 99.8, 88.7, 77.3, 77.0, 76.8, $75.0,72.8,72.8,71.5,62.0,29.0,19.1$.

IR (film, $\mathbf{~ c m}^{-1}$ ) 3420, 2884, 1581, 1373, 1264, 1198, 1079, 856. HRMS(ESI) Calc. for $\mathrm{C}_{15} \mathrm{H}_{20} \mathrm{O}_{5} \mathrm{SNa}(\mathrm{M}+\mathrm{Na})$ : 335.0924; found : 335.0928 .

\section{Part II. General Procedure for Regioselective Tetrahydropyranylation General Procedure I.}

An oven dried and nitrogen flushed $10 \mathrm{~mL}$ round bottom flask was charged with diol 1 (1 equiv.), (which was previously dried by azeotropic removal of moisture with toluene), anhydrous dichloromethane resulting in $0.04 \mathrm{M}$ concentration, and activated $4 \AA$ molecular sieves. This mixture was submerged in an ice-bath and 3,4-dihydro-2H-pyran (1.2 equiv.) was added, followed by $(R)-2 d(2 \mathrm{~mol} \%)$. The resulting white suspension was transferred into a Neslab CB 80 immersion cooler and stirred at $10{ }^{\circ} \mathrm{C}$ for 5 hours. When the reaction was completed as monitored by TLC, the reaction mixture was 
quenched with saturated aqueous $\mathrm{NaHCO}_{3}$ and extracted with ethyl acetate $(3 \times 10 \mathrm{~mL})$. The combined organic layer was dried with anhydrous $\mathrm{NaSO}_{4}$, filtered and concentrated in vacuo to form pale yellow oil as the crude product. The crude product was purified by flash column chromatography to afford the mono functionalized products as pale yellow oils.

\section{General Procedure 1I.}

An oven dried and nitrogen flushed $10 \mathrm{~mL}$ round bottom flask was charged with thioglycoside diol 1 (1 equiv.), (which was previously dried by azeotropic removal of moisture with toluene), anhydrous dichloromethane resulting in $0.04 \mathrm{M}$ concentration, and activated $4 \AA$ molecular sieves. This mixture was submerged in dry ice-acetonitrile bath and 3, 4-dihydro-2H-pyran (1.2 equiv.) was added, followed by $(R)-2 d$ ( $2 \mathrm{~mol} \%)$. The resulting reaction mixture was transferred into a Neslab CB 80 immersion cooler and stirred at $-20{ }^{\circ} \mathrm{C}$. When the reaction was completed as monitored by TLC, the reaction mixture was quenched with saturated aqueous $\mathrm{NaHCO}_{3}$, and extracted with ethyl acetate $(3 \times 10 \mathrm{~mL})$. The combined organic layer was dried with anhydrous $\mathrm{NaSO}_{4}$, filtered, and concentrated in vacuo to form pale yellow oil as the crude product. The crude product was purified by flash column chromatography to afford the respective mono functionalized products as pale yellow oils.

The assessment of the regioselectivity was accomplished by analysis of the ${ }^{1} \mathrm{H}$ NMR spectra of the products obtained by the CPA-controlled tetrahydropyranylation. This analysis was based on comparison with the independently synthesized C2 and C3 regioisomers of 3a. A typical procedure for the synthesis of $\mathrm{C} 2$ and $\mathrm{C} 3$ regioisomeric standards of 3a began using galactose diol 1a-I as starting material (see Scheme below). Mono acetylation of 1a-I afforded the $\mathrm{C} 2 / \mathrm{C} 3$ mixture of mono acetates, which were separated by a preparative TLC. The identity of the regioisomers was accessed by COSY NMR techniques (the protons next to the acetoxy group are typically shifted downfield). The pure $\mathrm{C} 2$ and $\mathrm{C} 3$ mono acetates were independently protected using 3,4-dihydro- $2 \mathrm{H}$ pyran (DHP) to afford the fully protected substrates, which were subsequently deacetylated using sodium methoxide in methanol, and each purified by flash column 
chromatography (1/1 Hexanes/Ethyl acetate) to afford the C2 and C3 standards as pale yellow oils. This protocol was followed in preparing $\mathrm{C} 2$ and $\mathrm{C} 3$ regio isomers of mono functionalized products $4 \mathbf{a}-\mathbf{4 e}$ by using their respective starting materials $\mathbf{1 a}-\mathbf{1 e}$.

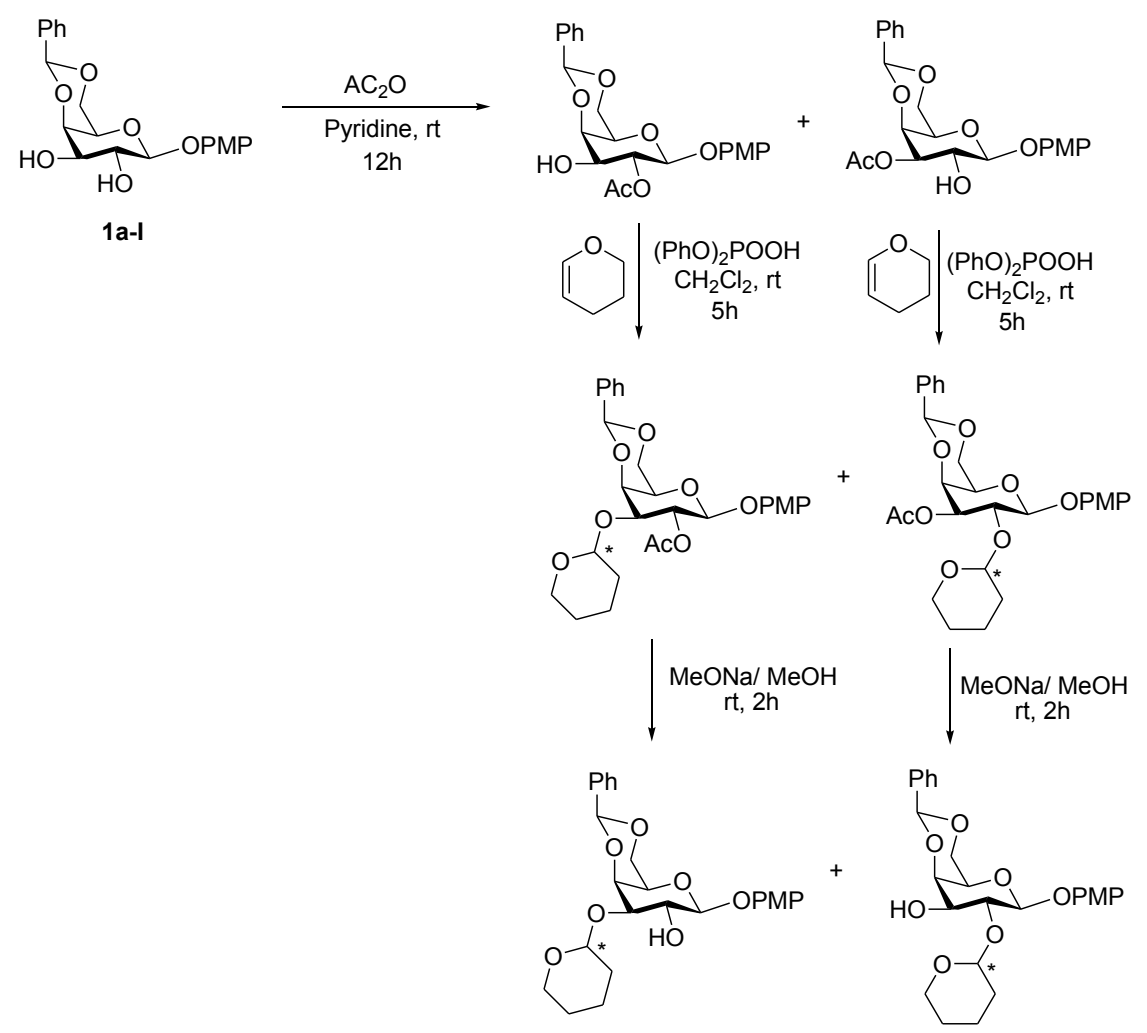

Part III. Regioselective Tetrahydropyranylation Reactions (Table 2)

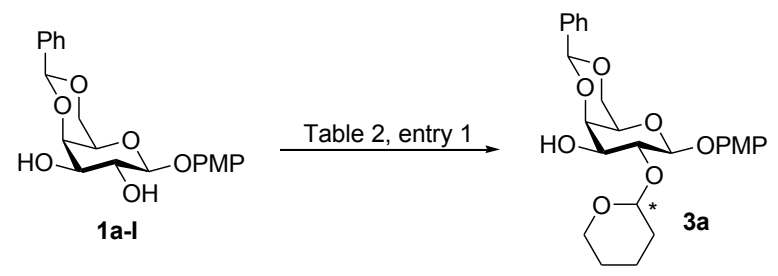

Galactose diol 1a-I was prepared according to the known literature procedure by Magnusson et al. ${ }^{3}$ Using substrate 1a-I (20 mg, $0.053 \mathrm{mmol}, 1$ equiv.) as the starting material, synthesis of 3a was accomplished by following general procedure I. This product was purified by flash column chromatography (2/1 Hexanes/Ethyl acetate $+1 \%$ triethylamine) to afford 3a as a pale yellow oil (18.9 $\mathrm{mg}, 78 \%$ ), and as an 8:1 mixture of diastereoisomers. 


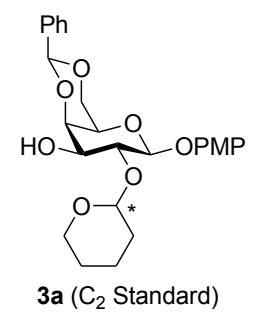

${ }^{1}$ H NMR (500 MHz, $\left.\mathbf{C D C l}_{3}\right) \delta 7.61-7.49(\mathrm{~m}, 4 \mathrm{H}), 7.43-7.30(\mathrm{~m}, 5 \mathrm{H}), 7.09-7.01(\mathrm{~m}$, 4H), $6.86-6.78(\mathrm{~m}, 4 \mathrm{H}), 5.58(\mathrm{~s}, 1 \mathrm{H}), 5.56(\mathrm{~s}, 1 \mathrm{H}), 4.85(\mathrm{~d}, J=10.0 \mathrm{~Hz}, 1 \mathrm{H}), 4.852(\mathrm{~d}, J$ $=10.0 \mathrm{~Hz}, 1 \mathrm{H}), 5.23(\mathrm{at}, J=3.4 \mathrm{~Hz}, 1 \mathrm{H}), 4.69(\mathrm{~d}, J=6.1 \mathrm{~Hz}, 1 \mathrm{H}), 4.35(\mathrm{dd}, J=12.4,1.3$ $\mathrm{Hz}, 2 \mathrm{H}), 4.30$ (d, $J=3.5 \mathrm{~Hz}, 1 \mathrm{H}), 4.22$ (d, $J=3.8 \mathrm{~Hz}, 1 \mathrm{H}), 4.19-4.12$ (m, 2H), $4.11-$ $4.04(\mathrm{~m}, 3 \mathrm{H}), 3.98(\mathrm{dd}, J=9.6,7.8 \mathrm{~Hz}, 1 \mathrm{H}), 3.82(\mathrm{td}, J=9.2,3.9 \mathrm{~Hz}, 1 \mathrm{H}), 3.78(\mathrm{~s}, 4 \mathrm{H})$, $3.73(\mathrm{dt}, J=9.6,3.3 \mathrm{~Hz}, 1 \mathrm{H}), 3.59-3.47$ (m, 4H), 2.85 (d, $J=9.1 \mathrm{~Hz}, 1 \mathrm{H}), 1.91-1.79$ $(\mathrm{m}, 4 \mathrm{H}), 1.78-1.67(\mathrm{~m}, 2 \mathrm{H}), 1.56-1.49(\mathrm{~m}, 6 \mathrm{H})$.

${ }^{13}$ C NMR (125 MHz, $\left.\mathbf{C D C l}_{3}\right) \delta 155.4,155.2,151.8,137.7,137.4,129.3,129.1,128.2$, 128.2 , 126.7, 126.6, 119.3, 118.8, 114.4, 102.5, 102.1, 101.7, 101.6, 101.5, 98.1, 79.4, 77.3, 77.0, 76.8, 75.5, 75.2, 74.0, 73.9, 71.1, 69.1, 69.0, 66.7, 66.5, 65.5, 62.1, 55.6, 31.3, 30.3, 25.4, 24.9, 21.3, 19.0 .

IR (film, $\mathbf{~ c m}^{-1}$ ) 3398, 2919, 2862,1634, 1507, 1452, 1369,1222, 1174 1167, 1077, 1053, 1027, 1005, 968, 913. HRMS(ESI) Calc. for $\mathrm{C}_{25} \mathrm{H}_{30} \mathrm{O}_{8} \mathrm{Na}(\mathrm{M}+\mathrm{Na}): 481.1833$; found : $481.1849 ;[\boldsymbol{\alpha}]^{26}{ }_{\mathbf{D}}=-72^{\circ}\left(c 0.12, \mathrm{CH}_{2} \mathrm{Cl}_{2}\right)$.

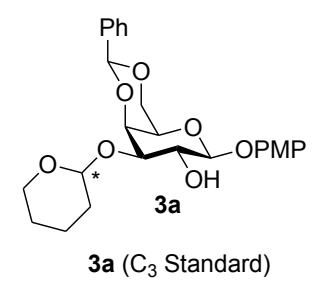

${ }^{1}$ H NMR (500 MHz, CDCl3 3 ) $\delta 7.57-7.49$ (m, 4H), $7.40-7.30$ (m, 5H), $7.13-7.05$ (m, 4H), $6.85-6.78(\mathrm{~m}, 4 \mathrm{H}), 5.57(\mathrm{~s}, 1 \mathrm{H}), 5.55(\mathrm{~s}, 1 \mathrm{H}), 4.97$ (at, $J=3.5 \mathrm{~Hz}, 1 \mathrm{H}), 4.84$ $(\mathrm{d}, J=7.7 \mathrm{~Hz}, 1 \mathrm{H}), 4.82(\mathrm{~d}, J=7.8 \mathrm{~Hz}, 1 \mathrm{H}), 4.75(\mathrm{dd}, J=5.4,2.8 \mathrm{~Hz}, 1 \mathrm{H}), 4.36(\mathrm{dd}, J=$ $5.2,2.5 \mathrm{~Hz}, 2 \mathrm{H}), 4.34$ (dd, $J=7.7,1.4 \mathrm{~Hz}, 1 \mathrm{H}), 4.28$ (d, $J=3.4 \mathrm{~Hz}, 1 \mathrm{H}), 4.19$ (dd, $J=$ 9.8, 8.0 Hz, 1H), $4.16-4.11(\mathrm{~m}, 1 \mathrm{H}), 4.08(\mathrm{dd}, J=12.4,1.3 \mathrm{~Hz}, 2 \mathrm{H}), 4.05-3.97(\mathrm{~m}$, 
2H), $3.77(\mathrm{~s}, 3 \mathrm{H}), 3.76(\mathrm{~s}, 3 \mathrm{H}), 3.72(\mathrm{dd}, J=9.9,3.5 \mathrm{~Hz}, 2 \mathrm{H}), 3.62-3.42(\mathrm{~m}, 5 \mathrm{H}), 2.64$ (s, $1 \mathrm{H}), 1.91-1.66(\mathrm{~m}, 7 \mathrm{H}), 1.57-1.48(\mathrm{~m}, 5 \mathrm{H})$.

${ }^{13}$ C NMR (125 MHz, $\left.\mathbf{C D C l}_{3}\right) \delta 155.4,155.35,151.3,151.2,137.9,137.7,128.9,128.8$, 128.1, 126.4, 126.3, 119.4, 119.2, 114.4, 114.3, 102.9, 102.7, 101.2, 101.0, 100.9, 97.8, 79.4, 78.3, 77.3, 77.0, 76.6, 76.1, 73.2, 69.4, 69.2, 69.1, 68.6, 66.9, 66.7, 63.7, 62.5, 55.6, $55.6,30.8,30.6,25.4,25.0,19.9,19.4$.

IR (film, $\mathbf{~ c m}^{-1}$ ) 3398, 2919, 2862,1634, 1507, 1452, 1369,1222, 1174 1167, 1077, 1053, 1027, 1005, 968, 913. HRMS(ESI) Calc. for $\mathrm{C}_{25} \mathrm{H}_{30} \mathrm{O}_{8} \mathrm{Na}(\mathrm{M}+\mathrm{Na}): 481.1833$; found : $481.1849 ;[\boldsymbol{\alpha}]^{26}{ }_{\mathbf{D}}=-51.4^{\circ}\left(c 0.12, \mathrm{CH}_{2} \mathrm{Cl}_{2}\right)$.

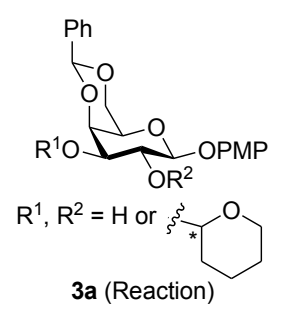

${ }^{1} \mathrm{H}$ NMR (500 MHz, $\left.\mathrm{CDCl}_{3}\right) \delta 7.59-7.53(\mathrm{~m}, 4 \mathrm{H}), 7.43-7.33(\mathrm{~m}, 6 \mathrm{H}), 7.13-7.10(\mathrm{~m}$, $0.3 \mathrm{H}), 7.10-7.05(\mathrm{~m}, 3 \mathrm{H}), 6.88-6.79(\mathrm{~m}, 4 \mathrm{H}), 5.59(\mathrm{~s}, 1 \mathrm{H}), 5.57(\mathrm{~s}, 0.6 \mathrm{H}), 5.57(\mathrm{~s}$, $0.1 \mathrm{H}$ ), 5.25 (at, $J=3.4 \mathrm{~Hz}, 0.6 \mathrm{H}$ ), 4.98 (at, $J=3.6 \mathrm{~Hz}, 0.08 \mathrm{H}$ ), $4.88-4.85(\mathrm{~m}, 2 \mathrm{H}), 4.84$ (d, $J=7.8 \mathrm{~Hz}, 0.1 \mathrm{H}), 4.77$ (dd, $J=5.4,2.7 \mathrm{~Hz}, 0.1 \mathrm{H}), 4.73-4.68(\mathrm{~m}, 1 \mathrm{H}), 4.37$ (d, $J=$ $12.4 \mathrm{~Hz}, 2 \mathrm{H}), 4.32$ (d, $J=3.2 \mathrm{~Hz}, 1 \mathrm{H}), 4.30$ (d, $J=3.8 \mathrm{~Hz}, 0.1 \mathrm{H}), 4.24$ (dd, $J=3.9,0.8$ $\mathrm{Hz}, 0.7 \mathrm{H}), 4.21-4.19(\mathrm{~m}, 0.3 \mathrm{H}), 4.19-4.15(\mathrm{~m}, 1 \mathrm{H}), 4.14(\mathrm{~s}, 0.2 \mathrm{H}), 4.13-4.10(\mathrm{~m}$, 2H), $4.10-4.08$ (m, 1H), $4.08-4.04$ (m, 1H), 4.00 (dd, $J=9.6,7.8 \mathrm{~Hz}, 1 \mathrm{H}), 3.84$ (dd, $J$ $=9.3,3.9 \mathrm{~Hz}, 0.6 \mathrm{H}), 3.79(\mathrm{~s}, 5 \mathrm{H}), 3.78(\mathrm{~d}, J=1.8 \mathrm{~Hz}, 1 \mathrm{H}), 3.75(\mathrm{dd}, J=9.6,3.7 \mathrm{~Hz}, 1 \mathrm{H})$, $3.59-3.51(\mathrm{~m}, 4 \mathrm{H}), 1.91-1.80(\mathrm{~m}, 3 \mathrm{H}), 1.79-1.72(\mathrm{~m}, 2 \mathrm{H}), 1.63-1.60(\mathrm{~m}, 2 \mathrm{H}), 1.54$ $(\mathrm{s}, 4 \mathrm{H})$.
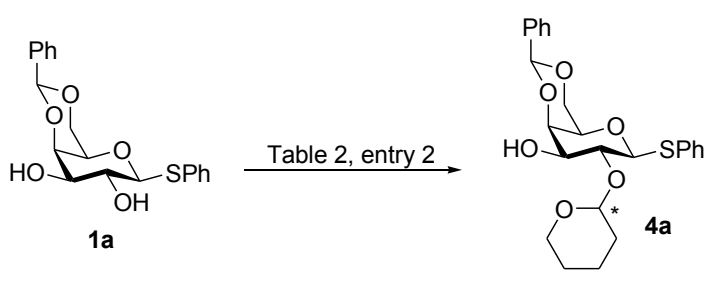

Galactose diol 1a was prepared according to the known literature procedure by Magnusson et al. ${ }^{3}$ Using the galactose diol $\mathbf{1 a}(20 \mathrm{mg}, 0.056 \mathrm{mmol}, 1$ equiv.) as the starting material, synthesis of $\mathbf{4 a}$ was accomplished by following general procedure II. 
This product was purified by flash column chromatography (1/1 Hexanes/Ethyl acetate + $1 \%$ triethylamine) to afford $4 \mathbf{a}$ as a pale yellow oil (18.5 $\mathrm{mg}, 74 \%$ ), and as an 8:1 mixture of diastereoisomers.

\section{Scaled up Synthesis of 4a}

An oven dried and nitrogen flushed $100 \mathrm{~mL}$ round bottom flask was charged with thioglycoside diol 1a (400 mg, $1.11 \mathrm{mmol}, 1$ equiv.), (which was previously dried by azeotropic removal of moisture with toluene), anhydrous dichloromethane $(26 \mathrm{~mL})$ and activated $4 \AA$ molecular sieves. This mixture was submerged in dry ice-acetonitrile bath and 3, 4-dihydro-2H-pyran ( $0.12 \mathrm{~mL}, 1.33 \mathrm{mmol}, 1.2$ equiv.) was added, followed by $(R)$-2d (20.8 $\mathrm{mg}, 0.022 \mathrm{mmol}, 2 \mathrm{~mol} \%)$. The resulting reaction mixture was transferred into a Neslab CB 80 immersion cooler and stirred at $-20{ }^{\circ} \mathrm{C}$. When the reaction was completed as monitored by TLC, the reaction mixture was quenched with saturated aqueous $\mathrm{NaHCO}_{3}$, and extracted with ethyl acetate $(3 \times 50 \mathrm{~mL})$. The combined organic layer was dried with anhydrous $\mathrm{NaSO}_{4}$, filtered, and concentrated in vacuo to form pale yellow oil as the crude product. The crude product was purified by flash column chromatography (1/1 Hexanes/Ethyl acetate $+1 \%$ triethylamine) to afford $\mathbf{4 a}$ as a pale yellow oil ( $0.35 \mathrm{~g}, 72 \%)$, and as an 8:1 mixture of diastereoisomers.

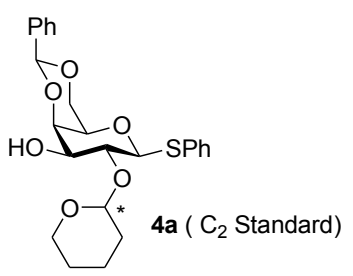

${ }^{1}$ H NMR (500 MHz, $\left.\mathbf{C D C l}_{3}\right) \delta 7.71-7.62(\mathrm{~m}, 4 \mathrm{H}), 7.52-7.48(\mathrm{~m}, 2 \mathrm{H}), 7.48-7.42(\mathrm{~m}$, 2H), $7.42-7.32(\mathrm{~m}, 6 \mathrm{H}), 7.29-7.26(\mathrm{~m}, 4 \mathrm{H}), 7.25-7.24(\mathrm{~m}, 1 \mathrm{H}), 5.54(\mathrm{~s}, 1 \mathrm{H}), 5.52(\mathrm{~s}$, $1 \mathrm{H}), 5.12(\mathrm{dd}, J=4.6,3.2 \mathrm{~Hz}, 1 \mathrm{H}), 4.62(\mathrm{~d}, J=6.4 \mathrm{~Hz}, 1 \mathrm{H}), 4.60(\mathrm{~d}, J=6.1 \mathrm{~Hz}, 1 \mathrm{H})$, 4.57 (d, $J=1.4 \mathrm{~Hz}, 1 \mathrm{H}), 4.40-4.33(\mathrm{~m}, 2 \mathrm{H}), 4.30$ (d, $J=2.8 \mathrm{~Hz}, 1 \mathrm{H}), 4.25-4.20$ (m, 2H), $4.18-4.13(\mathrm{~m}, 1 \mathrm{H}), 4.05$ (dd, $J=12.4,1.6 \mathrm{~Hz}, 1 \mathrm{H}), 4.02$ (dd, $J=12.4,1.7 \mathrm{~Hz}, 1 \mathrm{H})$, 3.94 (at, $J=9.2 \mathrm{~Hz}, 2 \mathrm{H}$ ), $3.80-3.74$ (m, 2H), 3.69 (ddd, $J=9.1,3.4,1.4 \mathrm{~Hz}, 1 \mathrm{H}$ ), $3.52-$ $3.50(\mathrm{~m}, 2 \mathrm{H}), 3.49-3.47(\mathrm{~m}, 1 \mathrm{H}), 3.40-3.36(\mathrm{~m}, 1 \mathrm{H}), 2.89(\mathrm{~d}, J=9.1 \mathrm{~Hz}, 1 \mathrm{H}), 1.95-$ 
$1.92(\mathrm{~m}, 1 \mathrm{H}), 1.88-1.83(\mathrm{~m}, 2 \mathrm{H}), 1.76-1.71(\mathrm{~m}, 2 \mathrm{H}), 1.67-1.64(\mathrm{~m}, 1 \mathrm{H}), 1.54-1.49$ $(\mathrm{m}, 5 \mathrm{H}), 1.30-1.26(\mathrm{~m}, 1 \mathrm{H})$.

${ }^{13}$ C NMR (125 MHz, $\left.\mathbf{C D C l}_{3}\right) \delta 137.8,137.5,133.2,132.8,132.6,132.6,129.3,129.1$, 128.9, 128.6, 128.2, 128.1, 127.7, 127.3, 126.8, 126.6, 102.6, 101.8, 101.5, 98.8, 86.0, 85.7, 78.1, 77.3, 77.0, 76.7, 75.8, 75.3, 75.1, 73.1, 72.87, 70.1, 69.8, 69.4, 69.2, 66.0, $63.3,31.2,307,29.7,25.3,24.8,21.6,19.6$.

IR (film, $\mathbf{~ c m}^{-1}$ ) 3410, 2927, 2848, 1583, 1454, 1436, 1357, 1266, 1161, 1097, 1071, 1025, 900. HRMS(ESI) Calc. for $\mathrm{C}_{24} \mathrm{H}_{28} \mathrm{O}_{6} \mathrm{SNa}(\mathrm{M}+\mathrm{Na})$ : 467.1499; found : 467.1514; $[\boldsymbol{\alpha}]^{26}{ }_{\mathbf{D}}=-35.9^{\circ}\left(c 0.11, \mathrm{CH}_{2} \mathrm{Cl}_{2}\right)$.

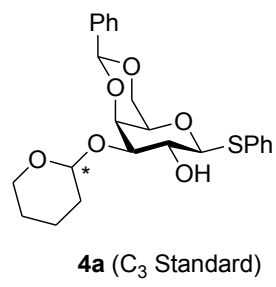

${ }^{1}$ H NMR (500 MHz, $\left.\mathbf{C D C l}_{3}\right) \delta 7.74-7.65(\mathrm{~m}, 3 \mathrm{H}), 7.47-7.38(\mathrm{~m}, 4 \mathrm{H}), 7.38-7.31(\mathrm{~m}$, $5 \mathrm{H}), 7.29-7.26(\mathrm{~m}, 3 \mathrm{H}), 7.25-7.17(\mathrm{~m}, 4 \mathrm{H}), 5.53(\mathrm{~s}, 1 \mathrm{H}), 5.50(\mathrm{~s}, 1 \mathrm{H}), 4.91(\mathrm{t}, J=5.0$ $\mathrm{Hz}, 1 \mathrm{H}), 4.68$ (dd, $J=6.0,2.7 \mathrm{~Hz}, 1 \mathrm{H}), 4.55$ (d, $J=9.5 \mathrm{~Hz}, 2 \mathrm{H}), 4.39$ (dd, $J=6.5,1.6$ $\mathrm{Hz}, 1 \mathrm{H}), 4.37(\mathrm{dd}, J=6.5,1.5 \mathrm{~Hz}, 1 \mathrm{H}), 4.34(\mathrm{~d}, J=2.7 \mathrm{~Hz}, 1 \mathrm{H}), 4.26(\mathrm{~d}, J=2.6 \mathrm{~Hz}, 1 \mathrm{H})$, $4.04(\mathrm{~d}, J=1.6 \mathrm{~Hz}, 1 \mathrm{H}), 4.02(\mathrm{~d}, J=1.6 \mathrm{~Hz}, 1 \mathrm{H}), 3.98(\mathrm{dd}, J=11.3,5.7 \mathrm{~Hz}, 1 \mathrm{H}), 3.96-$ 3.91 (m, 2H), 3.88 (at, $J=9.4 \mathrm{~Hz}, 1 \mathrm{H}), 3.83(\mathrm{~s}, 1 \mathrm{H}), 3.70$ (td, $J=9.2,3.3 \mathrm{~Hz}, 2 \mathrm{H}), 3.55$ (d, $J=1.0 \mathrm{~Hz}, 1 \mathrm{H}), 3.54-3.46(\mathrm{~m}, 3 \mathrm{H}), 2.50(\mathrm{~s}, 1 \mathrm{H}), 1.87-1.65(\mathrm{~m}, 7 \mathrm{H}), 1.53-1.45$ (m, 5H).

${ }^{13}$ C NMR (125 MHz, $\left.\mathbf{C D C l}_{3}\right) \delta$ 138.02, 137.85, 133.68, 133.44, 131.33, 130.90, 128.96, $128.89,128.87,128.72,128,76.99,76.74,76.30,73.67,70.24,70.06,69.39,69.31$, $66.88,66.19,64.10,62.58,30.82,30.57,25.33,24.92,20.24,19.38$.

IR (film, $\mathbf{~ c m}^{-1}$ ) 3410, 2927, 2848, 1583, 1454, .05, 127.94, 127.78, 126.49, 126.42, 101.28, 101.01, 100.94, 98.40, 87.40, 87.05, 80.58, 80.10, 77.251436, 1357, 1266, 1161, 1097, 1071, 1025, 900. HRMS(ESI) Calc. for $\mathrm{C}_{24} \mathrm{H}_{28} \mathrm{O}_{6} \mathrm{~S} \mathrm{Na}(\mathrm{M}+\mathrm{Na}): 467.1499$; found : 467.1514; $[\boldsymbol{\alpha}]^{\mathbf{2 6}}{ }_{\mathbf{D}}=-9.0^{\circ}\left(c 0.07, \mathrm{CH}_{2} \mathrm{Cl}_{2}\right)$. 

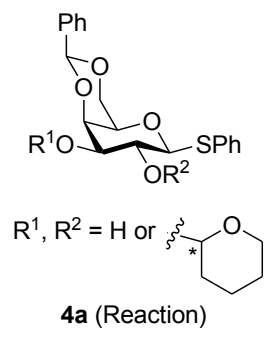

${ }^{1}$ H NMR (500 MHz, $\left.\mathbf{C D C l}_{3}\right) \delta 7.72-7.65(\mathrm{~m}, 2 \mathrm{H}), 7.55-7.49(\mathrm{~m}, 2 \mathrm{H}), 7.49-7.44(\mathrm{~m}$, 1H), $7.43-7.33(\mathrm{~m}, 3 \mathrm{H}), 7.33-7.25(\mathrm{~m}, 4 \mathrm{H}), 5.56(\mathrm{~s}, 0.7 \mathrm{H}), 5.54(\mathrm{~s}, 0.3 \mathrm{H}), 5.52(\mathrm{~s}$, $0.02 \mathrm{H}$ ), 5.36 (at, $J=4.6 \mathrm{~Hz}, 0.04 \mathrm{H}), 5.13$ (dd, $J=4.7,3.1 \mathrm{~Hz}, 0.3 \mathrm{H}), 4.66-4.59$ (m, 2H), $4.57(\mathrm{~d}, J=9.4 \mathrm{~Hz}, 0.05 \mathrm{H}), 4.40(\mathrm{dd}, J=12.4,1.6 \mathrm{~Hz}, 1 \mathrm{H}), 4.36(\mathrm{~d}, J=1.4 \mathrm{~Hz}$, $0.2 \mathrm{H}), 4.32(\mathrm{~d}, J=2.8 \mathrm{~Hz}, 0.7 \mathrm{H}), 4.27(\mathrm{~d}, J=2.6 \mathrm{~Hz}, 0.02 \mathrm{H}), 4.26-4.21(\mathrm{~m}, 1 \mathrm{H}), 4.17$ (dt, $J=11.4,5.9 \mathrm{~Hz}, 0.3 \mathrm{H}), 4.07(\mathrm{dd}, J=12.4,1.6 \mathrm{~Hz}, 0.7 \mathrm{H}), 4.03(\mathrm{dd}, J=12.4,1.6 \mathrm{~Hz}$, $0.4 \mathrm{H}$ ), 3.95 (t, $J=9.2 \mathrm{~Hz}, 1 \mathrm{H}), 3.89$ (d, $J=8.8 \mathrm{~Hz}, 0.04 \mathrm{H}$ ), 3.77 (at, $J=9.2 \mathrm{~Hz}, 1 \mathrm{H}), 3.71$ (ddd, $J=9.1,3.4,1.3 \mathrm{~Hz}, 0.7 \mathrm{H}), 3.57-3.47(\mathrm{~m}, 1 \mathrm{H}), 3.44-3.34(\mathrm{~m}, 0.7 \mathrm{H}), 2.92(\mathrm{~d}, J=$ $9.0 \mathrm{~Hz}, 0.3 \mathrm{H}), 1.97-1.94(\mathrm{~m}, 1 \mathrm{H}), 1.91-1.79(\mathrm{~m}, 1 \mathrm{H}), 1.79-1.72(\mathrm{~m}, 0.3 \mathrm{H}), 1.71-$ $1.60(\mathrm{~m}, 0,6 \mathrm{H}), 1.59-1.42(\mathrm{~m}, 4 \mathrm{H}), 1.27-1.24(\mathrm{~m}, 1 \mathrm{H})$.

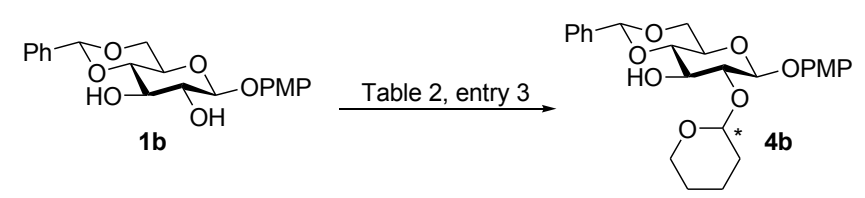

Diol 1b was prepared according to the known literature procedure by Bundle et al. ${ }^{4}$ Using glucose diol $1 \mathbf{b}$ (20 mg, $0.056 \mathrm{mmol}, 1$ equiv.) as the starting material, synthesis of 4b was accomplished by following general procedure I. This product was then purified by flash column chromatography (2/1 Hexanes/Ethyl acetate $+1 \%$ triethylamine) to afford $\mathbf{4 b}$ as a pale yellow oil (13.2 $\mathrm{mg}, 54 \%$ ), and as a 6:1 mixture of diastereoisomers.

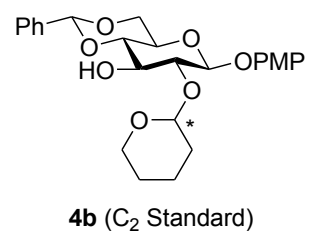

4. Bundle et al. J. Org. Chem. 2005, 65, $3064-3073$. 
${ }^{1}$ H NMR (500 MHz, $\left.\mathbf{C D C l}_{3}\right) \delta 7.56-7.48(\mathrm{~m}, 3 \mathrm{H}), 7.41-7.32(\mathrm{~m}, 5 \mathrm{H}), 7.03-6.98(\mathrm{~m}$, $3 \mathrm{H}), 6.88-6.83(\mathrm{~m}, 3 \mathrm{H}), 5.59(\mathrm{~s}, 1 \mathrm{H}), 4.94(\mathrm{~d}, J=10.0 \mathrm{~Hz}, 1 \mathrm{H}), 4.70(\mathrm{~d}, J=5.0 \mathrm{~Hz}, 1 \mathrm{H})$, $4.58(\mathrm{~s}, 1 \mathrm{H}), 4.38(\mathrm{dd}, J=12.8,6.4 \mathrm{~Hz}, 1 \mathrm{H}), 4.08$ (at, $J=5.0 \mathrm{~Hz}, 1 \mathrm{H}), 4.05$ (at, $J=5.0$ $\mathrm{Hz}, 1 \mathrm{H}), 3.90-3.82(\mathrm{~m}, 3 \mathrm{H}), 3.80(\mathrm{~s}, 4 \mathrm{H}), 3.70-3.64(\mathrm{~m}, 3 \mathrm{H}), 3.62(\mathrm{td}, J=9.8,5.1 \mathrm{~Hz}$, 2H), $3.55(\mathrm{td}, J=9.8,5.1 \mathrm{~Hz}, 2 \mathrm{H}), 1.64-1.56(\mathrm{~m}, 8 \mathrm{H}), 1.22-1.17(\mathrm{~m}, 11 \mathrm{H})$.

${ }^{13}$ C NMR (125 MHz, $\left.\mathbf{C D C l}_{3}\right) \delta 155.8,155.4,151.5,151.3,137.0,136.9,129.2,129.1$, $128.4,128.3,128.2,126.3,126.32,128.2,126.3,126.25,118.9,118.6,118.4,114.6$, $114.57,114.5,102.4,102.2,101.8,101.8,101.79,101.5,98.6,83.8,80.5,80.5,80.3$, 77.3, 77.0, 76.7, 74.1, 73.2, 72.1, 68.7, 68.7, 66.2, 66.0, 65.4, 63.3, 55.6, 31.1, 30.3, 29.7, 25.3, 24.9, 21.1, 19.6 .

IR (film, $\mathbf{c m}^{-\mathbf{1}}$ ) 3407, 2932, 2857, 1652, 1507, 1456, 1386, 1220, 1073, 1022.

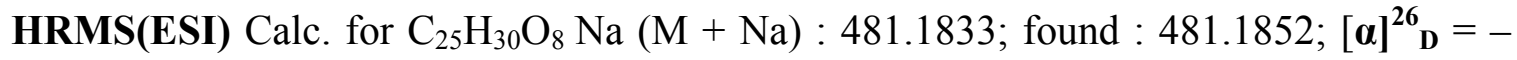
$0.8^{\circ}\left(c 0.5, \mathrm{CH}_{2} \mathrm{Cl}_{2}\right)$.

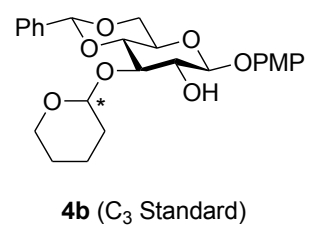

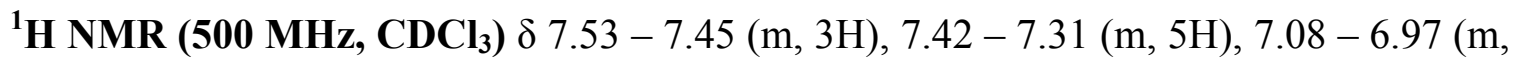
$3 \mathrm{H}), 6.88-6.78(\mathrm{~m}, 4 \mathrm{H}), 5.58(\mathrm{~s}, 1 \mathrm{H}), 5.56(\mathrm{~s}, 1 \mathrm{H}), 5.11$ (at, $J=3.8 \mathrm{~Hz}, 1 \mathrm{H}), 4.94(\mathrm{~d}, J=$ $7.5 \mathrm{~Hz}, 1 \mathrm{H}), 4.89$ (d, $J=7.8 \mathrm{~Hz}, 1 \mathrm{H}), 4.66$ (s, 1H), $4.63-4.59$ (m, 1H), 4.57 (s, 1H), $4.37(\mathrm{dt}, J=10.3,5.1 \mathrm{~Hz}, 2 \mathrm{H}), 4.09-4.05(\mathrm{~m}, 1 \mathrm{H}), 4.05-3.99(\mathrm{~m}, 2 \mathrm{H}), 3.84-3.82$ (m, 1H), $3.81-3.79(\mathrm{~m}, 1 \mathrm{H}), 3.78(\mathrm{~s}, 2 \mathrm{H}), 3.77$ (s, 3H), 3.73 (d, $J=7.6 \mathrm{~Hz}, 1 \mathrm{H}), 3.68$ (td, $J=$ 11.0, 10.0, 2.0 Hz, 2H), $3.56-3.47(\mathrm{~m}, 4 \mathrm{H}), 1.92-1.80(\mathrm{~m}, 5 \mathrm{H}), 1.77-1.65(\mathrm{~m}, 5 \mathrm{H})$, $1.59-1.51(\mathrm{~m}, 8 \mathrm{H})$.

${ }^{13}$ C NMR (125 MHz, $\left.\mathbf{C D C l}_{3}\right) \delta$ 155.6, 155.5 151.1, 151.0, 137.23, 137.2, 129.0, 128.9, 128.2, 128.1, 126.0, 125.99, 118.9, 118.8, 118.7, 114.6, 114.5, 103.0, 102.8, 102.2, 101.4, $101.3,98.3,82.9,79.1,79.0,77.3,77.0,76.7,75.8,74.9,73.0,68.7,68.7,68.66,67.0$, $66.6,65.3,62.9,55.6,55.60,31.0,30.2,25.3,24.9,21.1,19.5$. 
IR (film, $\mathbf{c m}^{-1}$ ) 3407, 2932, 2857, 1652, 1507, 1456, 1386, 1220, 1073, 1022. HRMS(ESI) Calc. for $\mathrm{C}_{25} \mathrm{H}_{30} \mathrm{O}_{8} \mathrm{Na}(\mathrm{M}+\mathrm{Na}): 481.1833$; found : 481.1843; $[\boldsymbol{\alpha}]^{\mathbf{2 6}}{ }_{\mathbf{D}}=-$ $25.8^{\circ}\left(c 0.11, \mathrm{CH}_{2} \mathrm{Cl}_{2}\right)$.

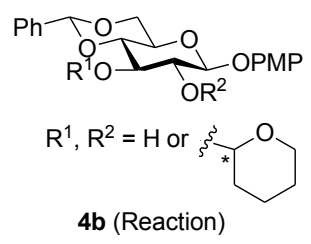

${ }^{1}$ H NMR (500 MHz, $\left.\mathbf{C D C l}_{3}\right) \delta 7.56-7.46(\mathrm{~m}, 4 \mathrm{H}), 7.42-7.33(\mathrm{~m}, 6 \mathrm{H}), 7.09-7.05(\mathrm{~m}$, $0.3 \mathrm{H}), 7.05-6.99(\mathrm{~m}, 4 \mathrm{H}), 6.88-6.83(\mathrm{~m}, 4 \mathrm{H}), 5.59(\mathrm{~s}, 1 \mathrm{H}), 5.58(\mathrm{~s}, 1 \mathrm{H}), 5.57(\mathrm{~s}, 0.7 \mathrm{H})$, 5.17 (at, $J=5.0 \mathrm{~Hz}, 0.7 \mathrm{H}), 5.13$ (at, $J=3.9 \mathrm{~Hz}, 0.1 \mathrm{H}), 5.03-4.99$ (m, $0.7 \mathrm{H}), 4.95$ (d, $J=$ $7.6 \mathrm{~Hz}, 1 \mathrm{H}), 4.91(\mathrm{~d}, J=7.8 \mathrm{~Hz}, 0.1 \mathrm{H}), 4.73-4.69(\mathrm{~m}, 1 \mathrm{H}), 4.67$ (s, $0.1 \mathrm{H}), 4.62(\mathrm{dd}, J=$ 10.0, $5.0 \mathrm{~Hz}, 0.1 \mathrm{H}), 4.59$ (s, 1H), $4.38(\mathrm{dd}, J=10.0,5.0 \mathrm{~Hz}, 2 \mathrm{H}), 4.11-4.02(\mathrm{~m}, 2 \mathrm{H})$, $3.99-3.92(\mathrm{~m}, 1 \mathrm{H}), 3.90-3.82(\mathrm{~m}, 3 \mathrm{H}), 3.80(\mathrm{~s}, 3 \mathrm{H}), 3.80$ (s, 3H), $3.79(\mathrm{~s}, 1 \mathrm{H}), 3.76-$ $3.70(\mathrm{~m}, 0.5 \mathrm{H}), 3.69-3.64(\mathrm{~m}, 3 \mathrm{H}), 3.63-3.58(\mathrm{~m}, 2 \mathrm{H}), 3.58-3.52(\mathrm{~m}, 2 \mathrm{H}), 3.19(\mathrm{~d}, J$ $=1.5 \mathrm{~Hz}, 1 \mathrm{H}), 3.08(\mathrm{~d}, J=2.3 \mathrm{~Hz}, 1 \mathrm{H}), 1.93-1.82(\mathrm{~m}, 3 \mathrm{H}), 1.81-1.71(\mathrm{~m}, 2 \mathrm{H}), 1.58-$ $1.49(\mathrm{~m}, 5 \mathrm{H})$.

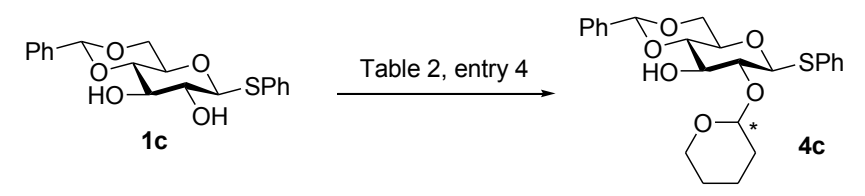

Compound $4 \mathbf{c}$ was prepared according to general procedure II, using glucose diol $1 \mathbf{c}^{5}$ (20 $\mathrm{mg}, 0.056 \mathrm{mmol}, 1$ equiv.) as the starting material. Crude $\mathbf{4 c}$ was purified by flash column chromatography (5/2 Hexanes/Ethyl acetate $+1 \%$ triethylamine) to afford pure $4 \mathbf{c}$ as a pale yellow oil (21.5 $\mathrm{mg}, 86 \%$ ), and as an 11:1 diastereoisomeric mixture.

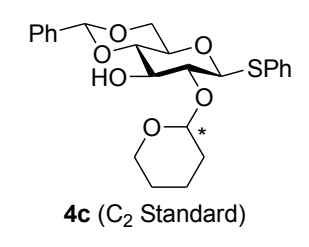

5. Soumik et al. Carbohydr. Res. 2008, 343, 2523 - 2529. 
${ }^{1}$ H NMR (500 MHz, CDCl $) \delta 7.55-7.46(\mathrm{~m}, 5 \mathrm{H}), 7.37-7.29(\mathrm{~m}, 6 \mathrm{H}), 5.56(\mathrm{~s}, 1 \mathrm{H})$, $5.53(\mathrm{~s}, 1 \mathrm{H}), 5.10(\mathrm{dd}, J=5.7,2.8 \mathrm{~Hz}, 1 \mathrm{H}), 4.89(\mathrm{~s}, 1 \mathrm{H}), 4.78(\mathrm{~d}, J=9.8 \mathrm{~Hz}, 1 \mathrm{H}), 4.73$ $(\mathrm{d}, J=9.8 \mathrm{~Hz}, 1 \mathrm{H}), 4.57(\mathrm{~s}, 1 \mathrm{H}), 4.46(\mathrm{~d}, J=7.3 \mathrm{~Hz}, 1 \mathrm{H}), 4.40-4.32$ (m, 2H), $4.19-$ $4.10(\mathrm{~m}, 2 \mathrm{H}), 3.96-3.92(\mathrm{~m}, 2 \mathrm{H}), 3.81-3.79(\mathrm{~m}, 1 \mathrm{H}), 3.75(\mathrm{dd}, J=9.1,7.7 \mathrm{~Hz}, 2 \mathrm{H})$, $3.64(\mathrm{~s}, 1 \mathrm{H}), 3.59-3.55(\mathrm{~m}, 2 \mathrm{H}), 3.53-3.47$ (m, 2H), 3.38 (at, $J=8.2 \mathrm{~Hz}, 2 \mathrm{H}), 3.33$ (d, $J=1.9 \mathrm{~Hz}, 1 \mathrm{H}), 1.86-1.80(\mathrm{~m}, 4 \mathrm{H}), 1.70-1.66(\mathrm{~m}, 2 \mathrm{H}), 1.52-1.48(\mathrm{~m}, 5 \mathrm{H}), 1.29-$ $1.27(\mathrm{~m}, 3 \mathrm{H})$.

${ }^{13}$ C NMR (125 MHz, $\left.\mathbf{C D C l}_{3}\right) \delta 136.9,133.2,132.2,132.2,129.2,129.0,128.9,128.3$, $128.2,128.0,127.7,126.3,126.2,102.7,101.8,101.8,98.7,87.4,86.9,83.3,80.4,80.3$, $80.3,77.0,76.7,76.5,75.2,73.4,70.2,70.1,68.6,65.5,64.2,31.9,31.0,30.6,29.7,25.2$, 24.8, 21.1, 20.1, 14.1.

IR (film, $\mathbf{c m}^{-1}$ ) 3376, 2927, 2848,1634, 1652, 1557, 1540, 1456, 1438, 1415, 1086, 1075, 1029. HRMS(ESI) Calc. for $\mathrm{C}_{24} \mathrm{H}_{28} \mathrm{O}_{6} \mathrm{SNa}(\mathrm{M}+\mathrm{Na})$ : 467.1499; found : 467.1516; $[\boldsymbol{\alpha}]^{26}{ }_{\mathbf{D}}=-3.3^{\circ}\left(c 0.03, \mathrm{CH}_{2} \mathrm{Cl}_{2}\right)$.

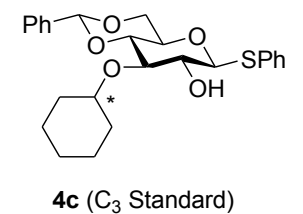

${ }^{1}$ H NMR (500 MHz, $\left.\mathbf{C D C l}_{3}\right) \delta 7.59$ - 7.52 (m, 4H), 7.50 - $7.43(\mathrm{~m}, 4 \mathrm{H}), 7.40-7.27$ (m, 12H), 5.55 (s, 1H), 5.53 (s, 1H), 5.08 (at, $J=4.0 \mathrm{~Hz}, 1 \mathrm{H}), 4.83(\mathrm{~s}, 1 \mathrm{H}), 4.67$ (d, $J=9.7$ $\mathrm{Hz}, 1 \mathrm{H}), 4.63$ (d, $J=9.8 \mathrm{~Hz}, 1 \mathrm{H}), 4.58-4.53(\mathrm{~m}, 2 \mathrm{H}), 4.38$ (dd, $J=10.5,4.9 \mathrm{~Hz}, 2 \mathrm{H})$, $4.08-4.01(\mathrm{~m}, 2 \mathrm{H}), 3.97(\mathrm{t}, J=8.9 \mathrm{~Hz}, 1 \mathrm{H}), 3.78(\mathrm{td}, J=10.1,2.8 \mathrm{~Hz}, 2 \mathrm{H}), 3.71$ (at, $J=$ $8.4 \mathrm{~Hz}, 1 \mathrm{H}), 3.60-3.56(\mathrm{~m}, 2 \mathrm{H}), 3.53-3.47(\mathrm{~m}, 6 \mathrm{H}), 3.14(\mathrm{~d}, J=1.7 \mathrm{~Hz}, 1 \mathrm{H}), 1.87-$ $1.82(\mathrm{~m}, 3 \mathrm{H}), 1.72-1.67(\mathrm{~m}, 2 \mathrm{H}), 1.53-1.50(\mathrm{~m}, 5 \mathrm{H}), 1.27-1.25(\mathrm{~m}, 3 \mathrm{H})$.

${ }^{13}$ C NMR (125 MHz, CDCl $) \delta$ 137.24, 137.21, 133.1, 133.0, 132.1, 131.4, 129.0, 129.0, $128.96,128.85,128.3,128.2,128.1,128.0,126.0,126.0,125.97,102.5,101.3,101.2$, 98.3, 89.0, 88.3, 84.3, 79.1, 79.0, 77.3, 77.0, 76.7, 73.1, 71.4, 71.1, 70.8, 68.6, 68.6, 65.6, 62.9, 53.4, 31.0, 30.2, 29.7, 25.2, 24.8, 21.2, 19.5 .

IR (film, cm ${ }^{-1}$ ) 3398, 2931, 2853, 1456, 1438, 1375, 1264, 1081, 1025, 908. HRMS(ESI) Calc. for $\mathrm{C}_{24} \mathrm{H}_{28} \mathrm{O}_{6} \mathrm{SNa}(\mathrm{M}+\mathrm{Na}): 467.1499$; found : 467.1518; $[\boldsymbol{\alpha}]^{\mathbf{2 6}}{ }_{\mathbf{D}}=-$ $10.1^{\circ}\left(c 0.08, \mathrm{CH}_{2} \mathrm{Cl}_{2}\right)$. 


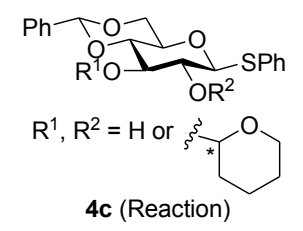

${ }^{1}$ H NMR (500 MHz, CDCl $) \delta 7.57-7.42(\mathrm{~m}, 6 \mathrm{H}), 7.37-7.27(\mathrm{~m}, 8 \mathrm{H}), 5.54(\mathrm{~s}, 1 \mathrm{H})$, $5.53(\mathrm{~s}, 0.1 \mathrm{H}), 5.51(\mathrm{~s}, 0.3 \mathrm{H}), 5.09(\mathrm{dd}, J=5.7,2.7 \mathrm{~Hz}, 0.2 \mathrm{H}), 5.06$ (at, $J=3.9 \mathrm{~Hz}$, $0.07 \mathrm{H}), 4.87$ (s, 1H), $4.80(\mathrm{~s}, 0.1 \mathrm{H}), 4.76(\mathrm{~d}, J=9.8 \mathrm{~Hz}, 0.2 \mathrm{H}), 4.71$ (d, $J=9.8 \mathrm{~Hz}, 1 \mathrm{H})$, $4.65(\mathrm{~d}, J=9.7 \mathrm{~Hz}, 0.2 \mathrm{H}), 4.61$ (d, $J=9.8 \mathrm{~Hz}, 0.1 \mathrm{H}), 4.54(\mathrm{~d}, J=5.9 \mathrm{~Hz}, 0.2 \mathrm{H}), 4.48-$ $4.42(\mathrm{~m}, 1 \mathrm{H}), 4.36(\mathrm{dd}, J=10.5,4.9 \mathrm{~Hz}, 1 \mathrm{H}), 4.32(\mathrm{~d}, J=4.8 \mathrm{~Hz}, 0.2 \mathrm{H}), 4.17-4.09$ (m, $0.2 \mathrm{H}), 4.03-3.96(\mathrm{~m}, 1 \mathrm{H}), 3.95-3.89(\mathrm{~m}, 0.2 \mathrm{H}), 3.83-3.73(\mathrm{~m}, 3 \mathrm{H}), 3.72-3.68(\mathrm{~m}$, $0.2 \mathrm{H}), 3.59-3.42(\mathrm{~m}, 5 \mathrm{H}), 3.38(\mathrm{dd}, J=9.6,8.3 \mathrm{~Hz}, 1 \mathrm{H}), 3.31(\mathrm{~s}, 0.2 \mathrm{H}), 3.11(\mathrm{~s}, 0.1 \mathrm{H})$, $1.93-1.78(\mathrm{~m}, 3 \mathrm{H}), 1.71-1.64(\mathrm{~m}, 0.4 \mathrm{H}), 1.53-1.48(\mathrm{~m}, 2 \mathrm{H}), 1.26-1.21(\mathrm{~m}, 3 \mathrm{H})$.

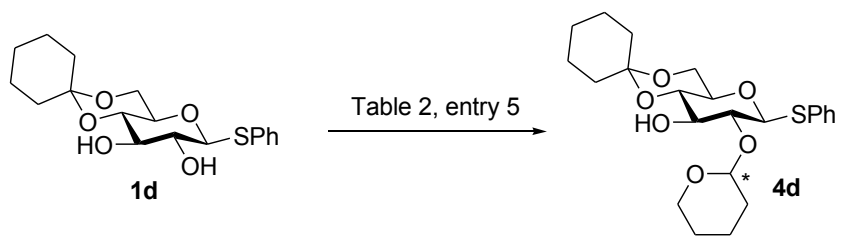

Compound 4d was prepared using general procedure II with glucose diol 1d (20 mg, 0.056 mmol, 1 equiv.) as starting material. Crude $4 \mathbf{d}$ was purified by flash column chromatography (2/1 Hexanes/Ethyl acetate $+1 \%$ triethylamine) to afford pure $\mathbf{4 d}$ as a pale yellow oil (20.5 mg, 82\%), and as a 6:1 diastereoisomeric mixture.

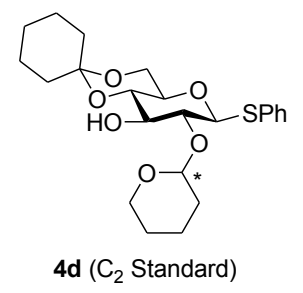

${ }^{1}$ H NMR (500 MHz, $\left.\mathbf{C D C l}_{3}\right) \delta 7.53-7.45(\mathrm{~m}, 3 \mathrm{H}), 7.35-7.26(\mathrm{~m}, 5 \mathrm{H}), 5.10(\mathrm{dd}, J=$ $5.5,2.7 \mathrm{~Hz}, 1 \mathrm{H}), 4.81(\mathrm{~s}, 1 \mathrm{H}), 4.72(\mathrm{~d}, J=9.6 \mathrm{~Hz}, 1 \mathrm{H}), 4.66(\mathrm{~d}, J=9.8 \mathrm{~Hz}, 1 \mathrm{H}), 4.45-$ $4.40(\mathrm{~m}, 1 \mathrm{H}), 4.18-4.11(\mathrm{~m}, 1 \mathrm{H}), 4.00(\mathrm{~d}, J=10.5 \mathrm{~Hz}, 1 \mathrm{H}), 3.96-3.89(\mathrm{~m}, 2 \mathrm{H}), 3.81-$ $3.75(\mathrm{~m}, 2 \mathrm{H}), 3.69(\mathrm{dd}, J=16.8,8.4 \mathrm{~Hz}, 1 \mathrm{H}), 3.63(\mathrm{dd}, J=10.4,8.2 \mathrm{~Hz}, 1 \mathrm{H}), 3.60-3.48$ 
(m, 3H), $3.36-3.27(\mathrm{~m}, 3 \mathrm{H}), 3.22(\mathrm{~s}, 1 \mathrm{H}), 2.06-1.99(\mathrm{~m}, 1 \mathrm{H}), 1.97-1.77(\mathrm{~m}, 7 \mathrm{H}), 1.70$ - $1.56(\mathrm{~m}, 10 \mathrm{H}), 1.48-1.40(\mathrm{~m}, 6 \mathrm{H}), 1.27-1.23(\mathrm{~m}, 6 \mathrm{H}), 0.90-0.84(\mathrm{~m}, 3 \mathrm{H})$.

${ }^{13} \mathbf{C}$ NMR (125 MHz, $\left.\mathbf{C D C l}_{3}\right) \delta$ 133.4, 133.0, 132.1, 132.0, 129.0, 128.8, 127.83, 127.5, 102.7, 99.8, 99.7, 98.6, 87.3, 86.8, 83.4, 77.2, 77.0, 76.7, 76.4, 75.8, 73.9, 72.1, 72.0, $71.4,71.2,65.5,64.1,61.3,61.3,37.8,37.8,31.0,30.6,29.7,29.6,29.3,27.7,27.7,25.6$, $25.5,25.2,24.8,22.9,22.6,22.5,21.1,20.0$.

IR (film, $\mathbf{c m}^{-1}$ ) 3424, 2932, 2853, 1439, 1364, 1270, 1101, 1075, 1025, 921, 906. HRMS(ESI) Calc. for $\mathrm{C}_{23} \mathrm{H}_{32} \mathrm{O}_{6} \mathrm{SNa}(\mathrm{M}+\mathrm{Na}): 459.1812$; found : 459.1821; $[\boldsymbol{\alpha}]^{\mathbf{2 6}}{ }_{\mathbf{D}}=-$ $22.2^{\circ}$ (c $\left.0.104, \mathrm{CH}_{2} \mathrm{Cl}_{2}\right)$.

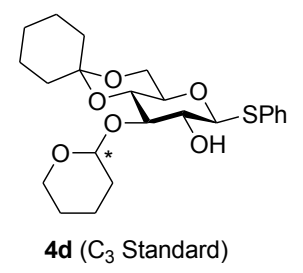

${ }^{1}$ H NMR (500 MHz, $\left.\mathbf{C D C l}_{3}\right) \delta 7.57-7.48(\mathrm{~m}, 3 \mathrm{H}), 7.34-7.26(\mathrm{~m}, 4 \mathrm{H}), 5.13$ (at, $J=3.6$ $\mathrm{Hz}, 1 \mathrm{H}), 4.60$ (d, $J=6.3 \mathrm{~Hz}, 1 \mathrm{H}), 4.56$ (d, $J=9.8 \mathrm{~Hz}, 1 \mathrm{H}), 4.50$ (s, 1H), $4.19-4.12$ (m, 1H), $4.05-3.99(\mathrm{~m}, 1 \mathrm{H}), 3.97-3.90(\mathrm{~m}, 2 \mathrm{H}), 3.84-3.76(\mathrm{~m}, 3 \mathrm{H}), 3.61-3.53(\mathrm{~m}, 3 \mathrm{H})$, $3.53-3.47(\mathrm{~m}, 1 \mathrm{H}), 3.46-3.27(\mathrm{~m}, 4 \mathrm{H}), 3.01(\mathrm{~d}, J=1.9 \mathrm{~Hz}, 1 \mathrm{H}), 2.25-2.10(\mathrm{~m}, 2 \mathrm{H})$, $1.91-1.75(\mathrm{~m}, 3 \mathrm{H}), 1.72-1.45(\mathrm{~m}, 22 \mathrm{H}), 1.40-1.25(\mathrm{~m}, 4 \mathrm{H})$.

${ }^{13} \mathbf{C}$ NMR (125 MHz, $\left.\mathbf{C D C l}_{3}\right) \delta 133.0,132.9,132.2,131.5,129.0,128.1,128.2,127.9$, 102.2, 99.7, 99.6, 97.4, 88.9, 88.2, 84.3, 77.2, 77.0, 76.7, 73.4, 72.3, 72.0, 71.4, 71.3, $70.9,65.3,62.4,61.6,61.6,37.9,37.8,31.1,30.2,29.7,27.8,27.7,25.6,25.6,25.4,24.9$, $22.9,22.7,22.4,22.4,21.1,19.2$.

IR (film, $\mathbf{~ c m}^{-1}$ ) 3407, 2936, 2862, 1584, 1481, 1439, 1362, 1264, 1172, 1156, 1106, 1075, 1025, 968. HRMS(ESI) Calc. for $\mathrm{C}_{23} \mathrm{H}_{32} \mathrm{O}_{6} \mathrm{SNa}(\mathrm{M}+\mathrm{Na}): 459.1812$; found : $459.1827 ;[\boldsymbol{\alpha}]^{26}{ }_{\mathbf{D}}=-22.2^{\circ}\left(c 0.122, \mathrm{CH}_{2} \mathrm{Cl}_{2}\right)$. 


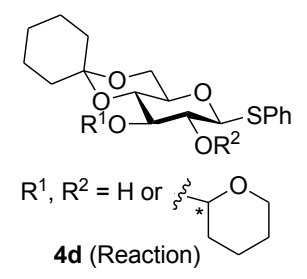

${ }^{1}$ H NMR (500 MHz, CDCl $) \delta 7.56-7.44(\mathrm{~m}, 4 \mathrm{H}), 7.35-7.25(\mathrm{~m}, 5 \mathrm{H}), 5.11$ (at, $J=3.6$ $\mathrm{Hz}, 0.1 \mathrm{H}), 5.08(\mathrm{dd}, J=5.6,2.8 \mathrm{~Hz}, 0.4 \mathrm{H}), 4.80$ (s, 1H), 4.70 (d, $J=9.6 \mathrm{~Hz}, 0.4 \mathrm{H}), 4.64$ $(\mathrm{d}, J=9.8 \mathrm{~Hz}, 1 \mathrm{H}), 4.59(\mathrm{~d}, J=9.7 \mathrm{~Hz}, 0.3 \mathrm{H}), 4.54(\mathrm{~d}, J=9.8 \mathrm{~Hz}, 0.1 \mathrm{H}), 4.49(\mathrm{~s}, 0.2 \mathrm{H})$, $4.43-4.38(\mathrm{~m}, 1 \mathrm{H}), 4.17-4.09(\mathrm{~m}, 0.5 \mathrm{H}), 4.01-3.95(\mathrm{~m}, 1 \mathrm{H}), 3.93(\mathrm{dd}, J=10.8,5.5$ $\mathrm{Hz}, 1 \mathrm{H}), 3.90-3.86(\mathrm{~m}, 0.4 \mathrm{H}), 3.83-3.71(\mathrm{~m}, 2 \mathrm{H}), 3.71-3.65(\mathrm{~m}, 0.4 \mathrm{H}), 3.65-3.58$ $(\mathrm{m}, 2 \mathrm{H}), 3.58-3.45(\mathrm{~m}, 3 \mathrm{H}), 3.44-3.35(\mathrm{~m}, 0.4 \mathrm{H}), 3.35-3.25(\mathrm{~m}, 3 \mathrm{H}), 3.22(\mathrm{~s}, 0.3 \mathrm{H})$, $2.99(\mathrm{~s}, 0.1 \mathrm{H}), 2.04-1.97(\mathrm{~m}, 1 \mathrm{H}), 1.94-1.73(\mathrm{~m}, 5 \mathrm{H}), 1.71-1.59(\mathrm{~m}, 5 \mathrm{H}), 1.54-1.48$ $(\mathrm{m}, 7 \mathrm{H}), 1.45-1.38(\mathrm{~m}, 4 \mathrm{H}), 1.27-1.23(\mathrm{~m}, 3 \mathrm{H})$.

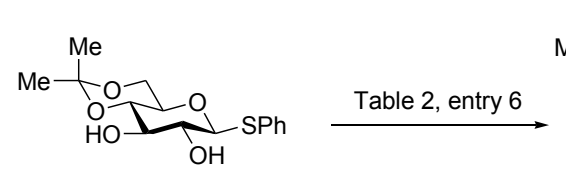

1e

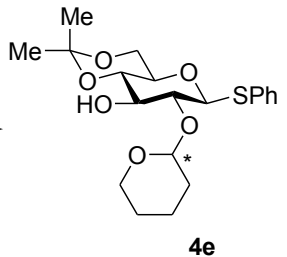

$4 \mathrm{e}$

Compound 4e was prepared according to general procedure II, using glucose diol 1e (20 mg, 0.056 mmol, 1 equiv.) as the starting material. Crude product $4 \mathbf{e}$ was purified by flash column chromatography (2/1 Hexanes/Ethyl acetate $+1 \%$ triethylamine $)$ to afford pure $4 \mathbf{e}$ as a pale yellow oil (19.5 mg, $77 \%$ ), and as a 10:1 diastereoisomeric mixture.

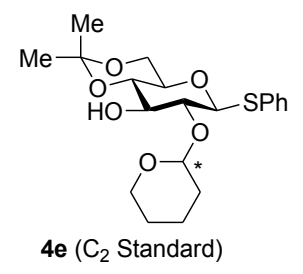

${ }^{1}$ H NMR (500 MHz, CDCl $) \delta 7.52-7.46(\mathrm{~m}, 3 \mathrm{H}), 7.35-7.26(\mathrm{~m}, 4 \mathrm{H}), 7.26(\mathrm{~s}, 1 \mathrm{H})$, $5.06(\mathrm{dd}, J=6.0,2.6 \mathrm{~Hz}, 1 \mathrm{H}), 4.87$ (s, 1H), 4.73 (d, $J=9.6 \mathrm{~Hz}, 1 \mathrm{H}), 4.67$ (d, $J=9.8 \mathrm{~Hz}$, $1 \mathrm{H}), 4.43(\mathrm{~d}, J=7.3 \mathrm{~Hz}, 1 \mathrm{H}), 4.13(\mathrm{dd}, J=10.8,5.1 \mathrm{~Hz}, 1 \mathrm{H}), 4.00$ (d, $J=10.4 \mathrm{~Hz}, 1 \mathrm{H})$, $3.94(\mathrm{ddd}, J=16.2,10.8,5.4 \mathrm{~Hz}, 2 \mathrm{H}), 3.85-3.73(\mathrm{~m}, 2 \mathrm{H}), 3.73-3.68(\mathrm{~m}, 1 \mathrm{H}), 3.67-$ 
$3.62(\mathrm{~m}, 1 \mathrm{H}), 3.62-3.46(\mathrm{~m}, 4 \mathrm{H}), 3.45-3.22(\mathrm{~m}, 4 \mathrm{H}), 1.96-1.76(\mathrm{~m}, 4 \mathrm{H}), 1.70-1.56$ (m, 4H), $1.52(\mathrm{~s}, 3 \mathrm{H}), 1.50(\mathrm{~s}, 2 \mathrm{H}), 1.45(\mathrm{~s}, 3 \mathrm{H}), 1.42(\mathrm{~s}, 2 \mathrm{H}), 1.25(\mathrm{~s}, 4 \mathrm{H})$.

${ }^{13} \mathbf{C}$ NMR (125 MHz, $\left.\mathbf{C D C l}_{3}\right) \delta 133.4,133.0,132.1,131.9,129.0,128.8,127.9,127.6$, 102.8, 99.7, 99.6, 98.6, 87.35, 86.8, 83.4, 77.2, 77.0, 76.73, 76.5, 75.4, 73.8, 73.0, 72.95, $71.1,71.0,65.6,64.5,62.0,31.2,30.6,29.7,29.0,25.2,24.8,21.1,20.3,19.1,19.0$.

IR (film, $\mathbf{c m}^{-1}$ ) 3424, 2923, 2853, 1584, 1481, 1437, 1375, 1264, 1200, 1165, 1128, 1075, 1027, 858. HRMS(ESI) Calc. for $\mathrm{C}_{20} \mathrm{H}_{28} \mathrm{O}_{6} \mathrm{SNa}(\mathrm{M}+\mathrm{Na}): 419.1499$; found : $419.1509 ;[\boldsymbol{\alpha}]^{\mathbf{2 6}}{ }_{\mathbf{D}}=-16.4^{\circ}\left(c 0.1, \mathrm{CH}_{2} \mathrm{Cl}_{2}\right)$.

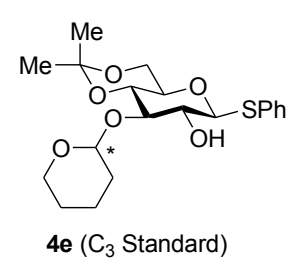

${ }^{1}$ H NMR (500 MHz, $\mathbf{C D C l}_{3}$ ) $\delta 7.57-7.47(\mathrm{~m}, 3 \mathrm{H}), 7.36-7.27$ (m, 4H), 5.02 (at, $J=3.8$ Hz, 1H), 4.72 (s, 1H), 4.62 (at, $J=7.0 \mathrm{~Hz}, 1 \mathrm{H}), 4.57$ (d, $J=9.8 \mathrm{~Hz}, 1 \mathrm{H}), 4.53$ (d, $J=6.0$ Hz, 1H), $4.11-4.05$ (m, 1H), 4.02 (dd, $J=14.3,3.1 \mathrm{~Hz}, 1 \mathrm{H}), 3.98-3.92(\mathrm{~m}, 2 \mathrm{H}), 3.80-$ $3.73(\mathrm{~m}, 3 \mathrm{H}), 3.55$ (ddd, $J=17.0,15.2,8.7 \mathrm{~Hz}, 3 \mathrm{H}), 3.50-3.44(\mathrm{~m}, 2 \mathrm{H}), 3.43-3.37$ (m, 1H), $3.37-3.31$ (m, 1H), 3.28 (dt, $J=16.1,7.8 \mathrm{~Hz}, 1 \mathrm{H}), 3.04$ (s, 1H), $1.88-1.74$ (m, $3 \mathrm{H}), 1.74-1.60(\mathrm{~m}, 4 \mathrm{H}), 1.59-1.50(\mathrm{~m}, 5 \mathrm{H}), 1.47$ (d, $J=6.5 \mathrm{~Hz}, 3 \mathrm{H}), 1.46(\mathrm{~s}, 2 \mathrm{H}), 1.39$ (s, 3H), 1.38 (s, 2H).

${ }^{13} \mathbf{C}$ NMR (125 MHz, $\left.\mathbf{C D C l}_{3}\right) \delta 132.9,132.8,132.3,131.7,129.0,128.8,128.2,127.9$, 102.3, 99.6, 99.5, 99.5, 98.1, 89.0, 88.3, 84.4, 77.7, 77.3, 77.0, 76.8, 76.6, 73.1, 72.1, $72.1,71.8,71.6,71.5,65.5,62.6,62.2,62.2,31.0,30.7,30.2,29.1,29.0,25.4,25.4,24.9$, $21.2,19.5,19.2,19.1$.

IR (film, $\mathbf{c m}^{-1}$ ) 3407, 2993, 2941, 2884, 1584, 1481, 1441, 1375, 1264, 1202, 1171, 1119, 1079, 1024, 970 908. HRMS(ESI) Calc. for $\mathrm{C}_{20} \mathrm{H}_{28} \mathrm{O}_{6} \mathrm{SNa}(\mathrm{M}+\mathrm{Na})$ : 419.1499; found : $419.1511 ;[\boldsymbol{\alpha}]^{\mathbf{2 6}} \mathbf{D}=-124.9^{\circ}\left(c 0.12, \mathrm{CH}_{2} \mathrm{Cl}_{2}\right)$. 


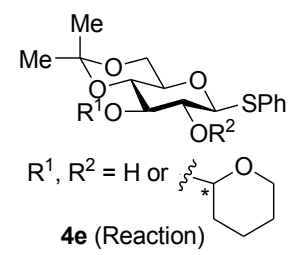

${ }^{1}$ H NMR (500 MHz, CDCl $) \delta 7.52-7.43(\mathrm{~m}, 3 \mathrm{H}), 7.33-7.25(\mathrm{~m}, 4 \mathrm{H}), 5.04$ (dd, $J=$ 6.0, $2.7 \mathrm{~Hz}, 0.2 \mathrm{H}), 5.01(\mathrm{~s}, 1 \mathrm{H}), 4.85$ (s, $0.7 \mathrm{H}), 4.71$ (d, $J=9.6 \mathrm{~Hz}, 0.3 \mathrm{H}), 4.65$ (d, $J=9.8$ $\mathrm{Hz}, 1 \mathrm{H}), 4.55(\mathrm{~d}, J=9.8 \mathrm{~Hz}, 0.03 \mathrm{H}), 4.44-4.39(\mathrm{~m}, 1 \mathrm{H}), 4.11(\mathrm{dd}, J=9.8,5.7 \mathrm{~Hz}$, $0.3 \mathrm{H}), 4.01-3.96(\mathrm{~m}, 1 \mathrm{H}), 3.94$ (dd, $J=10.8,5.4 \mathrm{~Hz}, 1 \mathrm{H}), 3.92-3.88(\mathrm{~m}, 0.2 \mathrm{H}), 3.81-$ $3.72(\mathrm{~m}, 2 \mathrm{H}), 3.71-3.66(\mathrm{~m}, 0.2 \mathrm{H}), 3.65-3.56(\mathrm{~m}, 2 \mathrm{H}), 3.56-3.47(\mathrm{~m}, 2 \mathrm{H}), 3.45-$ $3.40(\mathrm{~m}, 0.07 \mathrm{H}), 3.36-3.24(\mathrm{~m}, 2 \mathrm{H}), 1.94-1.81(\mathrm{~m}, 2 \mathrm{H}), 1.78(\mathrm{dd}, J=11.9,8.7 \mathrm{~Hz}$, $0.4 \mathrm{H}), 1.68-1.62(\mathrm{~m}, 0.5 \mathrm{H}), 1.61-1.52(\mathrm{~m}, 3 \mathrm{H}), 1.50(\mathrm{~s}, 4 \mathrm{H}), 1.47(\mathrm{~d}, J=6.8 \mathrm{~Hz}, 1 \mathrm{H})$, $1.43(\mathrm{~s}, 3 \mathrm{H}), 1.40(\mathrm{~s}, 1 \mathrm{H}), 1.27-1.20(\mathrm{~m}, 2 \mathrm{H})$.

\section{Regioselective Acetalization (Table 3)}

\section{General Procedure A.}

An oven dried and nitrogen flushed $10 \mathrm{~mL}$ round bottom flask was charged with thioglycoside diol 1 (1 equiv.), (which was previously dried by azeotropic removal of moisture with toluene), anhydrous dichloromethane resulting in $0.04 \mathrm{M}$ concentration, and activated $4 \AA$ molecular sieves. This mixture was submerged in a dry ice/acetone bath and 1-methoxycyclohexene (1.2 equiv.) was added, followed by $(R)-\mathbf{2 d}(2 \mathrm{~mol} \%)$. The resulting reaction mixture was transferred into a Neslab CB 80 immersion cooler and stirred at $-50{ }^{\circ} \mathrm{C}$ overnight. When the reaction was completed as indicated by TLC, the reaction mixture was quenched with triethylamine, and concentrated in vacuo to form the crude product. This crude product was purified by flash column chromatography to afford pale yellow oil.

\section{General Procedure B}

An oven dried and nitrogen flushed $10 \mathrm{~mL}$ round bottom flask was charged with thioglycoside diol 1 (1 equiv.), (which was previously dried by azeotropic removal of moisture with toluene), anhydrous dichloromethane resulting in $0.04 \mathrm{M}$ concentration and activated $4 \AA$ molecular sieves. This mixture was submerged in a dry ice/acetone bath and 2-methoxypropene (1.2 equiv.) followed by $(R)$-2d ( 2 mol\%) was added. The resulting 
reaction mixture was transferred into a Neslab CB 80 immersion cooler and stirred at -78 ${ }^{\circ} \mathrm{C}$ overnight. When the reaction was completed as indicated by $\mathrm{TLC}$, the reaction mixture was quenched with triethylamine, and concentrated in vacuo to form the crude product. This crude product was purified by flash column chromatography to afford pale yellow oil.

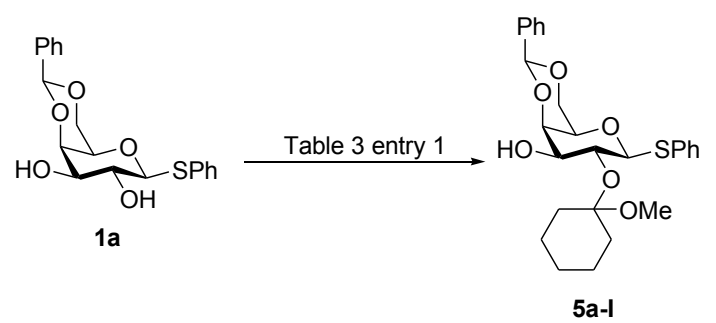

Using galactose diol $\mathbf{1 a}^{3}$ (20 mg, $0.056 \mathrm{mmol}, 1$ equiv.) as the starting material, synthesis of 5a-I was accomplished by following general procedure A. This product was purified by flash column chromatography (1/1 Hexanes/Ethyl acetate $+2 \%$ triethylamine) to afford 5a-I as a pale yellow oil $(20.5 \mathrm{mg}, 76 \%, \mathrm{C} 2: \mathrm{C} 3=10: 1)$.

${ }^{1}$ H NMR (500 MHz, CD $\mathbf{3}$ OD) $\delta 7.67-7.61(\mathrm{~m}, 2 \mathrm{H}), 7.60-7.55(\mathrm{~m}, 2 \mathrm{H}), 7.45-7.35$ (m, 3H), $7.27-7.19(\mathrm{~m}, 3 \mathrm{H}), 5.64(\mathrm{~s}, 1 \mathrm{H}), 4.72(\mathrm{~d}, J=9.3 \mathrm{~Hz}, 1 \mathrm{H}), 4.34(\mathrm{dd}, J=3.5,0.7$ $\mathrm{Hz}, 1 \mathrm{H}), 4.24$ (dd, $J=12.4,1.6 \mathrm{~Hz}, 1 \mathrm{H}), 4.17-4.08$ (m, 2H), 3.73 (dd, $J=8.8,3.5 \mathrm{~Hz}$, $1 \mathrm{H}), 3.62(\mathrm{~d}, J=1.0 \mathrm{~Hz}, 1 \mathrm{H}), 3.34(\mathrm{~s}, 3 \mathrm{H}), 1.94-1.81(\mathrm{~m}, 1 \mathrm{H}), 1.75-1.63(\mathrm{~m}, 3 \mathrm{H}), 1.49$ $-1.43(\mathrm{~m}, 1 \mathrm{H}), 1.40-1.34(\mathrm{~m}, 3 \mathrm{H}), 1.26-1.23(\mathrm{~m}, 1 \mathrm{H}), 0.93-0.86(\mathrm{~m}, 1 \mathrm{H})$.

${ }^{13}$ C NMR (125 MHz, CD 3 OD) $\delta 138.3,134.0,130.9,128.5,128.5,127.7,126.6,126.2$, $101.5,100.9,85.8,75.4,73.5,69.97,69.5,68.8,48.1,47.9,47.8,47.6,47.4,47.2$, 47.1, $47.0,33.0,32.7,24.8,22.3,22.2$.

IR (film, $\mathbf{c m}^{-1}$ ) 3371, 2927, 2857, 1581 1456, 1367, 1263, 1158, 1101, 1042, 1027, 998. HRMS(ESI) Calc. for $\mathrm{C}_{26} \mathrm{H}_{32} \mathrm{O}_{6} \mathrm{SNa}(\mathrm{M}+\mathrm{Na}): 495.1812$; found : 495.1814; $[\boldsymbol{\alpha}]^{\mathbf{2 6}}{ }_{\mathbf{D}}=-$ $36.6^{\circ}\left(c 0.32, \mathrm{CH}_{2} \mathrm{Cl}_{2}\right.$ ).

Determination of the $\mathrm{C} 2 / \mathrm{C} 3$ ratios was performed by acetylating the crude reaction mixture and analyzing the COSY NMR spectrum of the acetylated 5a-I (acetylation result in significant shift of the proton next to the acetoxy group to 4.2-4.6 ppm region). 


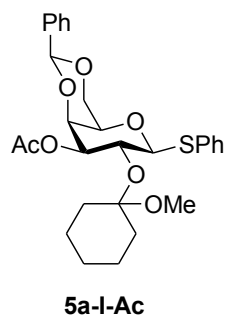

$\mathbf{R}_{\mathbf{f}}=0.3($ Hexane/Ethyl acetate, $2 / 1+1 \%$ Triethylamine $)$

${ }^{1}$ H NMR (500 MHz, CD 3 OD) $\delta 7.69-7.64(\mathrm{~m}, 2 \mathrm{H}), 7.62-7.57(\mathrm{~m}, 1 \mathrm{H}), 7.56-7.50$ (m, 2H), $7.49-7.35$ (m, 6H), $7.31-7.17$ (m, 5H), 5.59 (s, 1H), 5.59 (s, 0.4H), 5.19 (at, $J$ $=9.8 \mathrm{~Hz}, 0.4 \mathrm{H}), 5.00(\mathrm{dd}, J=9.1,3.7 \mathrm{~Hz}, 1 \mathrm{H}), 4.85(\mathrm{~d}, J=9.3 \mathrm{~Hz}, 0.4 \mathrm{H}), 4.76(\mathrm{~d}, J=9.3$ Hz, 1H), 4.39 (d, $J=3.0 \mathrm{~Hz}, 0.4 \mathrm{H}), 4.37$ (dd, $J=3.7,0.8 \mathrm{~Hz}, 1 \mathrm{H}), 4.30$ (t, $J=9.8 \mathrm{~Hz}$, $1 \mathrm{H}), 4.27-4.21(\mathrm{~m}, 2 \mathrm{H}), 4.16-4.08(\mathrm{~m}, 2 \mathrm{H}), 3.70(\mathrm{~d}, J=1.0 \mathrm{~Hz}, 1 \mathrm{H}), 3.68(\mathrm{~d}, J=1.0$ Hz, 0.4H), 3.25 (s, 0.3H), 3.22 (s, 1H), 3.12 (s, 3H), 2.08 (s, 1H), 2.07 (s, 3H), $1.89-$ $1.83(\mathrm{~m}, 1 \mathrm{H}), 1.78-1.74(\mathrm{~m}, 1 \mathrm{H}), 1.63-1.58(\mathrm{~m}, 2 \mathrm{H}), 1.54-1.47(\mathrm{~m}, 2 \mathrm{H}), 1.44-1.37$ (m, 4H), $1.31-1.29(\mathrm{~m}, 3 \mathrm{H}), 1.17-1.11(\mathrm{~m}, 2 \mathrm{H}), 0.92-0.86(\mathrm{~m}, 2 \mathrm{H})$.

${ }^{13}$ C NMR (125 MHz, CD $_{3}$ OD) $\delta 170.7,169.9,138.2,133.8,132.4,131.1,128.6,128.5$, 128.4, 128.4, 127.7, 127.62, 127.2, 126.6, 126.2, 126.1, 117.7, 101.8, 101.4, 101.0, 100.7, $86.5,85.1,75.8,75.5,74.7,74.2,71.0,69.7,69.5,68.9,68.7,68.0,66.4,33.5,33.3,33.2$, $33.0,25.0,22.7,22.6,22.4,22.2,19.8,19.8$.

IR (film, $\mathbf{~ c m}^{-1}$ ) 2927, 2857, 1748, 1452, 1371, 1239, 1167, 1095, 1055. HRMS(ESI) Calc. for $\mathrm{C}_{28} \mathrm{H}_{34} \mathrm{O}_{7} \mathrm{SNa}(\mathrm{M}+\mathrm{Na})$ : 537.1917; found : 537.1923; $[\boldsymbol{\alpha}]^{\mathbf{2 6}} \mathbf{\mathbf { b }}=9.6^{\circ}$ (c 0.18, $\mathrm{CH}_{2} \mathrm{Cl}_{2}$ ).

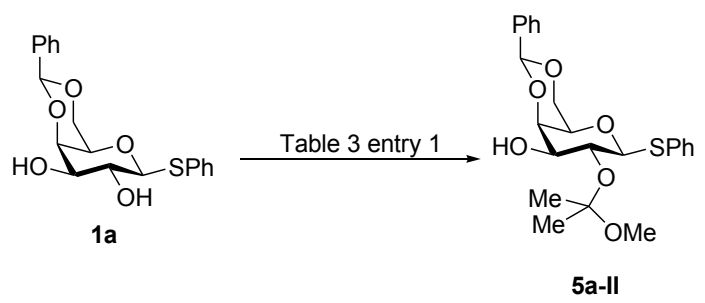

Using galactose diol $\mathbf{1 a}^{3}$ (20 $\mathrm{mg}, 0.056 \mathrm{mmol}, 1$ equiv.) as the starting material, preparation of 5a-II was accomplished by following general procedure B. This product 
was purified by flash column chromatography (1/1 Hexanes/Ethyl acetate $+1 \%$ triethylamine ) to afford 5a-II as a pale yellow oil (22.2 $\mathrm{mg}, 93 \%, \mathrm{C} 2: \mathrm{C} 3=20: 1)$.

${ }^{1}$ H NMR (500 MHz, $\left.\mathbf{C D C l}_{3}\right) \delta 7.70-7.62(\mathrm{~m}, 2 \mathrm{H}), 7.54(\mathrm{dd}, J=7.5,1.6 \mathrm{~Hz}, 2 \mathrm{H}), 7.42$ - $7.33(\mathrm{~m}, 3 \mathrm{H}), 7.25-7.18(\mathrm{~m}, 3 \mathrm{H}), 5.58(\mathrm{~s}, 1 \mathrm{H}), 5.16(\mathrm{~s}, 1 \mathrm{H}), 4.60(\mathrm{~d}, J=9.3 \mathrm{~Hz}, 1 \mathrm{H})$, $4.37(\mathrm{dd}, J=12.3,1.1 \mathrm{~Hz}, 1 \mathrm{H}), 4.31(\mathrm{~d}, J=3.2 \mathrm{~Hz}, 1 \mathrm{H}), 4.17-4.02(\mathrm{~m}, 2 \mathrm{H}), 3.65(\mathrm{dd}, J$ $=8.6,3.0 \mathrm{~Hz}, 1 \mathrm{H}), 3.50(\mathrm{~s}, 1 \mathrm{H}), 3.31(\mathrm{~s}, 3 \mathrm{H}), 1.43(\mathrm{~s}, 3 \mathrm{H}), 1.14(\mathrm{~d}, J=18.0 \mathrm{~Hz}, 3 \mathrm{H})$.

${ }^{13}$ C NMR (125 MHz, $\left.\mathbf{C D C l}_{3}\right) \delta 138.0,133.4,131.7,129.0,128.8,128.1,127.1,126.6$, $101.9,101.4,86.0,77.3,77.0,76.8,75.4,73.8,70.9,70.1,69.3,49.7,25.0,23.6$.

IR (film, cm ${ }^{-1}$ ) 3382, 2989, 2923, 2857, 1471, 1456, 1439, 1375, 1156, 1099, 1044. HRMS(ESI) Calc. for $\mathrm{C}_{23} \mathrm{H}_{28} \mathrm{O}_{6} \mathrm{SNa}(\mathrm{M}+\mathrm{Na}): 455.1499$; found : 455.1509; $[\boldsymbol{\alpha}]^{\mathbf{2 6}}{ }_{\mathbf{D}}=-$ $5.4^{\circ}\left(c 0.02, \mathrm{CH}_{2} \mathrm{Cl}_{2}\right)$.

Determination of the $\mathrm{C} 2 / \mathrm{C} 3$ ratios was performed by acetylating the crude reaction mixture and analyzing the COSY NMR spectrum of the acetylated 5a-II.

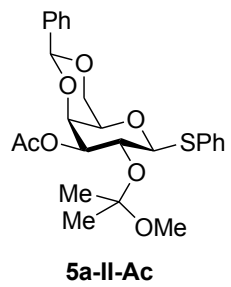

$\mathbf{R}_{\mathbf{f}}=0.3$ (Hexane/Ethyl acetate, $5 / 2+1 \%$ Triethylamine)

${ }^{1}$ H NMR (500 MHz, $\left.\mathbf{C D C l}_{3}\right) \delta 7.70-7.63(\mathrm{~m}, 2 \mathrm{H}), 7.56-7.49(\mathrm{~m}, 2 \mathrm{H}), 7.46-7.37$ (m, $3 \mathrm{H}), 7.25-7.18(\mathrm{~m}, 3 \mathrm{H}), 5.51(\mathrm{~s}, 1 \mathrm{H}), 4.92(\mathrm{dd}, J=9.2,3.6 \mathrm{~Hz}, 1 \mathrm{H}), 4.65(\mathrm{~d}, J=9.3 \mathrm{~Hz}$, 1H), 4.38 (dd, $J=12.4,1.5 \mathrm{~Hz}, 1 \mathrm{H}), 4.33$ (d, $J=3.0 \mathrm{~Hz}, 1 \mathrm{H}), 4.26$ (at, $J=9.3 \mathrm{~Hz}, 1 \mathrm{H}$ ), $4.03(\mathrm{dd}, J=12.4,1.6 \mathrm{~Hz}, 1 \mathrm{H}), 3.57$ (d, $J=0.8 \mathrm{~Hz}, 1 \mathrm{H}), 3.21(\mathrm{~s}, 3 \mathrm{H}), 2.11(\mathrm{~s}, 3 \mathrm{H}), 1.34$ $(\mathrm{s}, 3 \mathrm{H}), 1.20(\mathrm{~s}, 3 \mathrm{H})$. 
${ }^{13}$ C NMR (125 MHz, $\left.\mathbf{C D C l}_{3}\right) \delta 170.8,137.9,133.3,131.9,129.0,128.7,128.1,127.2$, 126.4, 101.8, 100.9, 87.1, 77.3, 77.0, 76.7, 75.0, 74.0, 69.6, 69.1, 67.6, 49.1, 29.7, 25.4, 24.9, 21.2.

IR (film, $\mathbf{c m}^{\mathbf{- 1}}$ ) 2984, 2923 2857, 1745, 1456, 1371, 1237, 1097, 1055. HRMS(ESI) Calc. for $\mathrm{C}_{25} \mathrm{H}_{30} \mathrm{O}_{7} \mathrm{SNa}(\mathrm{M}+\mathrm{Na})$ : 497.1604; found : 497.1619; $[\boldsymbol{\alpha}]^{\mathbf{2 6}}{ }_{\mathbf{D}}=-5.4^{\circ}$ (c 0.25, $\mathrm{CH}_{2} \mathrm{Cl}_{2}$ ).

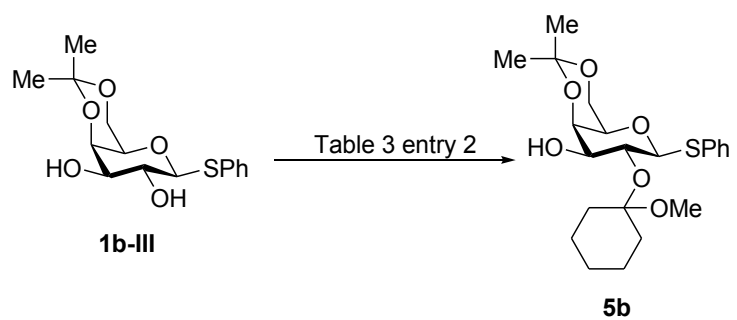

Using galactose diol 1b-III ${ }^{6}$ (20 $\mathrm{mg}, 0.064 \mathrm{mmol}, 1$ equiv.) as the starting material, synthesis of $\mathbf{5 b}$ was accomplished by following general procedure A. This product was purified by flash column chromatography $(2 / 1$ Hexanes/Ethyl acetate $+1 \%$ triethylamine) to afford $\mathbf{5 b}$ as a pale yellow oil (13.8 $\mathrm{mg}, 51 \%, \mathrm{C} 2: \mathrm{C} 3=25: 1)$.

${ }^{1}$ H NMR (500 MHz, C $\left.\mathbf{C}_{6} \mathbf{D}_{6}\right) \delta 7.83-7.74(\mathrm{~m}, 2 \mathrm{H}), 7.09-7.01(\mathrm{~m}, 2 \mathrm{H}), 6.97-6.90(\mathrm{~m}$, 1H), 5.09 (s, 1H), 4.51 (d, $J=9.2 \mathrm{~Hz}, 1 \mathrm{H}), 4.39$ (at, $J=9.0 \mathrm{~Hz}, 1 \mathrm{H}), 3.93$ (d, $J=3.4 \mathrm{~Hz}$, $1 \mathrm{H}), 3.75(\mathrm{dd}, J=12.6,1.7 \mathrm{~Hz}, 1 \mathrm{H}), 3.45(\mathrm{dd}, J=12.6,2.1 \mathrm{~Hz}, 1 \mathrm{H}), 3.37$ (ddd, $J=8.7$, 3.4, $1.4 \mathrm{~Hz}, 1 \mathrm{H}), 3.03(\mathrm{~s}, 3 \mathrm{H}), 2.51(\mathrm{~s}, 1 \mathrm{H}), 1.77-1.66(\mathrm{~m}, 2 \mathrm{H}), 1.64-1.57(\mathrm{~m}, 1 \mathrm{H})$, $1.53(\mathrm{~s}, 4 \mathrm{H}), 1.31$ (at, $J=16.9 \mathrm{~Hz}, 3 \mathrm{H}), 1.20(\mathrm{~s}, 4 \mathrm{H}), 1.15-1.08(\mathrm{~m}, 1 \mathrm{H}), 1.09-1.00$ (m, $1 \mathrm{H})$.

${ }^{13}$ C NMR (125 MHz, $\left.\mathbf{C}_{6} \mathbf{D}_{6}\right) \delta 134.8,131.8,128.6,127.9,127.8,127.7,127.5,126.7$, $101.5,98.7,86.3,74.1,70.2,70.1,68.3,62.6,47.2,33.3,33.2,29.4,25.1,22.7,22.4$, 18.3.

IR (film, $\mathbf{c m}^{-1}$ ) 3385, 2932, 2857, 1441, 1378, 1285, 1154, 1097, 1057, 1042. HRMS(ESI) Calc. for $\mathrm{C}_{22} \mathrm{H}_{32} \mathrm{O}_{6} \mathrm{SNa}(\mathrm{M}+\mathrm{Na}): 447.1812$; found : 447.1828; $[\boldsymbol{\alpha}]^{\mathbf{2 6}}{ }_{\mathbf{D}}=$ $1.3^{\circ}\left(c 0.08, \mathrm{CH}_{2} \mathrm{Cl}_{2}\right)$.

6. Pedretti et al. Tetrahedron. 1990, 46, $77-88$. 
Determination of the $\mathrm{C} 2 / \mathrm{C} 3$ ratios was performed by acetylating the crude reaction mixture and analyzing the COSY NMR spectrum of the acetylated $\mathbf{5 b}$.

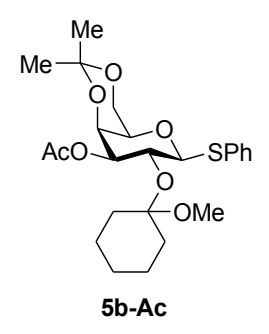

$\mathbf{R}_{\mathbf{f}}=0.3($ Hexane/Ethyl acetate, $2 / 1+1 \%$ Triethylamine $)$

${ }^{1} \mathbf{H}$ NMR (500 MHz, $\left.\mathbf{c}_{\mathbf{6}} \mathbf{d}_{\mathbf{6}}\right) \delta 7.80(\mathrm{~d}, J=8.3 \mathrm{~Hz}, 2 \mathrm{H}), 7.10-7.04(\mathrm{~m}, 3 \mathrm{H}), 6.99-6.92$ (m, 1H), 4.94 (dd, $J=8.6,3.6 \mathrm{~Hz}, 1 \mathrm{H}), 4.59-4.46(\mathrm{~m}, 2 \mathrm{H}), 4.00$ (d, $J=3.7 \mathrm{~Hz}, 1 \mathrm{H})$, 3.67 (d, $J=12.6 \mathrm{~Hz}, 1 \mathrm{H}), 3.33$ (d, $J=12.6 \mathrm{~Hz}, 1 \mathrm{H}), 3.14$ (s, 3H), 2.47 (s, 1H), $2.00-$ $1.91(\mathrm{~m}, 1 \mathrm{H}), 1.88(\mathrm{~s}, 3 \mathrm{H}), 1.87-1.80(\mathrm{~m}, 1 \mathrm{H}), 1.65-1.59(\mathrm{~m}, 1 \mathrm{H}), 1.47(\mathrm{~s}, 3 \mathrm{H}), 1.40-$ $1.36(\mathrm{~m}, 2 \mathrm{H}), 1.34-1.24(\mathrm{~m}, 5 \mathrm{H}), 1.23-1.18(\mathrm{~m}, 1 \mathrm{H}), 1.09(\mathrm{~s}, 3 \mathrm{H}), 1.06-0.99(\mathrm{~m}, 1 \mathrm{H})$. ${ }^{13}$ C NMR (125 MHz, $\left.\mathbf{C}_{6} \mathbf{D}_{6}\right) \delta 169.8,134.5,132.0,128.6,127.9,127.8,127.6,127.5$, $127.4,126.8,101.5,98.4,87.2,74.8,69.3,67.2,67.0,62.3,47.4,33.8,33.7,29.8,29.2$, 25.4, 23.0, 20.7, 18.4 .

IR (film, $\mathbf{c m}^{-1}$ ) 2927, 2871, 1748, 1450, 1373, 1242, 1180, 1147, 1095, 1057, 974, 917. HRMS(ESI) Calc. for $\mathrm{C}_{24} \mathrm{H}_{34} \mathrm{O}_{7} \mathrm{SNa}(\mathrm{M}+\mathrm{Na}): 489.1917$; found : 489.1930; $[\boldsymbol{\alpha}]^{\mathbf{2 6}}{ }_{\mathbf{D}}=$ $14.2^{\circ}\left(c 0.127, \mathrm{CH}_{2} \mathrm{Cl}_{2}\right)$.

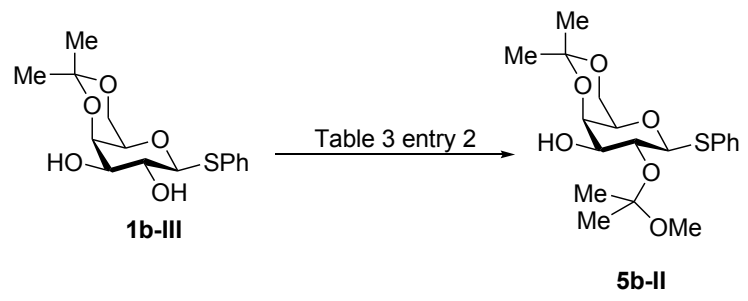

Using galactose diol $\mathbf{1 b}$-III ${ }^{6}$ (20 $\mathrm{mg}, 0.064 \mathrm{mmol}, 1$ equiv.) as the starting material, synthesis of $\mathbf{5 b}$-II was accomplished by following general procedure B. This product was purified by flash column chromatography ( $2 / 1$ Hexanes/Ethyl acetate $+1 \%$ triethylamine) to afford $\mathbf{5 b}$-II as a pale yellow oil $(20.6 \mathrm{mg}, 84 \%, \mathrm{C} 2: \mathrm{C} 3=20: 1)$. 
${ }^{1} \mathbf{H}$ NMR (500 MHz, $\left.\mathbf{C}_{6} \mathbf{D}_{6}\right) \delta 7.84-7.77(\mathrm{~m}, 2 \mathrm{H}), 7.11-7.04(\mathrm{~m}, 2 \mathrm{H}), 6.99-6.92(\mathrm{~m}$, 1H), $4.98(\mathrm{~d}, J=1.3 \mathrm{~Hz}, 1 \mathrm{H}), 4.50$ (d, $J=9.3 \mathrm{~Hz}, 1 \mathrm{H}), 4.35$ (at, $J=9.0 \mathrm{~Hz}, 1 \mathrm{H}), 3.91$ (d, $J=2.8 \mathrm{~Hz}, 1 \mathrm{H}), 3.75(\mathrm{dd}, J=12.6,1.7 \mathrm{~Hz}, 1 \mathrm{H}), 3.48-3.40(\mathrm{~m}, 2 \mathrm{H}), 2.90(\mathrm{~s}, 3 \mathrm{H}), 2.49$ $(\mathrm{d}, J=0.9 \mathrm{~Hz}, 1 \mathrm{H}), 1.54(\mathrm{~s}, 3 \mathrm{H}), 1.22(\mathrm{~s}, 3 \mathrm{H}), 1.20$ (s, 3H), 1.09 (s, 3H).

${ }^{13}$ C NMR (125 MHz, $\left.\mathbf{C}_{6} \mathbf{D}_{6}\right) \delta 134.7,131.6,128.6,127.8,127.6,127.4,126.7,101.7$, 98.4, 86.2, 74.0, 71.4, 69.9, 68.2, 62.6, 49.1, 29.8, 29.4, 24.8, 23.3, 18.3.

IR (film, cm ${ }^{-1}$ ) 3398, 2989, 2923, 1486, 1380, 1277, 1200, 1141, 1096, 1055, 1042. HRMS(ESI) Calc. for $\mathrm{C}_{19} \mathrm{H}_{28} \mathrm{O}_{8} \mathrm{SNa}(\mathrm{M}+\mathrm{Na}): 407.1499$; found : 407.1512; $[\boldsymbol{\alpha}]^{\mathbf{2 6}}{ }_{\mathbf{D}}=-$ $0.42^{\circ}\left(c 0.24, \mathrm{CH}_{2} \mathrm{Cl}_{2}\right)$.

Determination of the $\mathrm{C} 2 / \mathrm{C} 3$ ratios was performed by acetylating the crude reaction mixture and analyzing the COSY NMR spectrum of the acetylated $\mathbf{5 b}$-II.

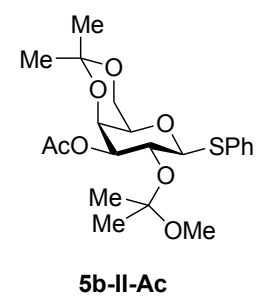

${ }^{1}$ H NMR (500 MHz, C $\left.\mathbf{C}_{\mathbf{6}} \mathbf{D}_{6}\right) \delta 7.85-7.75(\mathrm{~m}, 2 \mathrm{H}), 7.11-7.04(\mathrm{~m}, 2 \mathrm{H}), 6.99-6.93(\mathrm{~m}$, $1 \mathrm{H}), 4.94(\mathrm{dd}, J=9.0,3.7 \mathrm{~Hz}, 1 \mathrm{H}), 4.51$ (d, $J=9.3 \mathrm{~Hz}, 1 \mathrm{H}), 4.44$ (at, $J=9.1 \mathrm{~Hz}, 1 \mathrm{H}$ ), $3.98(\mathrm{dd}, J=3.5,0.5 \mathrm{~Hz}, 1 \mathrm{H}), 3.66(\mathrm{dd}, J=12.5,2.0 \mathrm{~Hz}, 1 \mathrm{H}), 3.32(\mathrm{dd}, J=12.5,2.0 \mathrm{~Hz}$, 1H), 3.18 (s, 3H), 2.45 (d, J=0.9 Hz, 1H), 1.86 (s, 3H), 1.45 (s, 3H), 1.34 (s, 3H), 1.19 $(\mathrm{s}, 3 \mathrm{H}), 1.07$ (s, 3H).

${ }^{13}$ C NMR (125 MHz, C $\left.6 \mathbf{D}_{6}\right) \delta 169.7,134.4,132.0,128.6,127.9,127.8,127.6,127.4$, $126.9,101.6,98.4,87.1,74.8,69.3,68.3,67.2,62.3,52.9,48.6,29.8,29.2,25.3,24.8$, 20.6, 18.4 .

IR (film, $\mathbf{c m}^{-1}$ ) 2993, 2927 1745, 1441, 1380, 1244, 1202, 1180, 1147, 1093, 1058. . HRMS(ESI) Calc. for $\mathrm{C}_{21} \mathrm{H}_{30} \mathrm{O}_{7} \mathrm{SNa}(\mathrm{M}+\mathrm{Na}): 449.1604$; found : 449.1610; $[\boldsymbol{\alpha}]^{\mathbf{2 6}} \mathbf{D}=$ $10.7^{\circ}$ ( c $0.23, \mathrm{CH}_{2} \mathrm{Cl}_{2}$ ). 


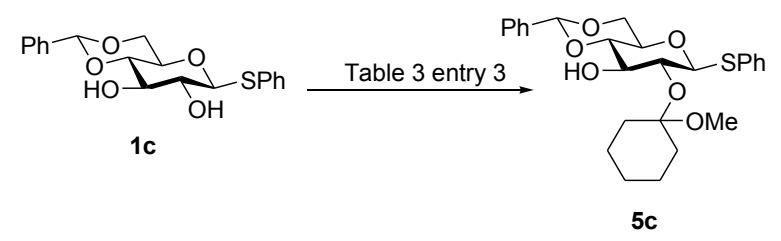

Using diol $\mathbf{1} \mathbf{c}^{5}$ (20 mg, $0.056 \mathrm{mmol}, 1$ equiv.) as the starting material, preparation of $\mathbf{5 c}$ was accomplished by following general procedure A. This product was purified by flash column chromatography $(7 / 1$ Hexanes/Ethyl acetate $+2 \%$ triethylamine $)$ to afford $\mathbf{5 c}$ as a pale yellow oil $(18.6 \mathrm{mg}, 69 \%, \mathrm{C} 2: \mathrm{C} 3=25: 1)$.

\section{Scaled up Synthesis of $5 \mathrm{c}$}

An oven dried and nitrogen flushed $250 \mathrm{~mL}$ round bottom flask was charged with thioglycoside diol $1 \mathbf{c c}^{5}$ (1 g, $2.78 \mathrm{mmol}, 1$ equiv.), (which was previously dried by azeotropic removal of moisture with toluene), anhydrous dichloromethane $(65 \mathrm{~mL})$ and activated $4 \AA$ molecular sieves. This mixture was submerged in dry ice/acetone bath and 1-methoxycylohexene ( $0.42 \mathrm{~mL}, 3.34 \mathrm{mmol}, 1.2$ equiv.) was added, followed by $(R)$-2d (52 $\mathrm{mg}, 0.056 \mathrm{mmol}, 2 \mathrm{~mol} \%$ ). The resulting reaction mixture was transferred into a Neslab CB 80 immersion cooler and stirred at $-50{ }^{\circ} \mathrm{C}$. When the reaction was completed as monitored by TLC, the reaction mixture was quenched with triethylamine, filtered through celite, and concentrated in vacuo to form the crude product. The crude product was purified by flash column chromatography (7/1 Hexanes/Ethyl acetate $+2 \%$ triethylamine) to afford $\mathbf{5 c}$ as a pale yellow foam $(1.24 \mathrm{~g}, 95 \%, \mathrm{C} 2: \mathrm{C} 3=>25: 1)$.

${ }^{1} \mathrm{H}$ NMR (500 MHz, CD $\left.{ }_{3} \mathrm{OD}\right) \delta 7.76(\mathrm{~s}, 0.02 \mathrm{H}), 7.56-7.52(\mathrm{~m}, 0.1 \mathrm{H}), 7.51-7.43(\mathrm{~m}$, $5 \mathrm{H}), 7.41-7.30(\mathrm{~m}, 6 \mathrm{H}), 7.29-7.23(\mathrm{~m}, 1 \mathrm{H}), 5.59(\mathrm{~s}, 1 \mathrm{H}), 5.54(\mathrm{~s}, 0.02 \mathrm{H}), 4.85(\mathrm{~d}, J=$ $8.5 \mathrm{~Hz}, 1 \mathrm{H}$ ), 4.26 (dd, $J=10.0,5.0 \mathrm{~Hz}, 1 \mathrm{H}), 3.83-3.71(\mathrm{~m}, 3 \mathrm{H}), 3.59$ (at, $J=9.2 \mathrm{~Hz}$, $1 \mathrm{H}), 3.56-3.48(\mathrm{~m}, 1 \mathrm{H}), 3.34(\mathrm{~s}, 3 \mathrm{H}), 3.18(\mathrm{~s}, 0.1 \mathrm{H}), 2.88-2.80(\mathrm{~m}, 0.2 \mathrm{H}), 1.98-1.79$ (m, 2H), $1.74-1.70(\mathrm{~m}, 1 \mathrm{H}), 1.67-1.60(\mathrm{~m}, 2 \mathrm{H}), 1.55-1.45(\mathrm{~m}, 2 \mathrm{H}), 1.43-1.35(\mathrm{~m}$, $2 \mathrm{H}), 1.29-1.25(\mathrm{~m}, 1 \mathrm{H}), 1.14(\mathrm{t}, J=7.3 \mathrm{~Hz}, 1 \mathrm{H})$.

${ }^{13}$ C NMR (125 MHz, CD ${ }_{3}$ OD) $\delta 147.3,146.5,137.6,134.0,132.2,131.3,130.9,128.6$, 128.5, 127.6, 127.6, 127.0, 126.1, 125.9, 101.6, 101.6, 101.5, 101.4, 87.9, 87.0, 79.7, 79.6, 74.9, 74.3, 72.5, 70.2, 69.7, 68.2, 48.1, 47.9, 47.7, 47.6, 47.4, 47.2, 47.1, 45.9, 43.2, $36.6,36.1,33.8,33.0,32.9,31.0,30.8,29.2,25.0,24.9,22.3,22.2$. 
IR (film, $\mathbf{c m}^{-1}$ ) 3380, 2931, 2857, 1581, 1557, 1541, 1463, 1367, 1272, 1250, 1149, 1090, 1014, 917. HRMS(ESI) Calc. for $\mathrm{C}_{26} \mathrm{H}_{32} \mathrm{O}_{6} \mathrm{SNa}(\mathrm{M}+\mathrm{Na}): 495.1812$; found : $495.1826 ;[\boldsymbol{\alpha}]^{26}{ }_{\mathbf{D}}=-27.5^{\circ}\left(c 0.35, \mathrm{CH}_{2} \mathrm{Cl}_{2}\right)$.

Determination of the $\mathrm{C} 2 / \mathrm{C} 3$ ratios was performed by acetylating the crude reaction mixture and analyzing the COSY NMR spectrum of the acetylated $\mathbf{5 c}$.

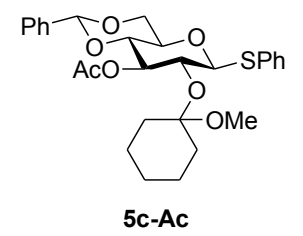

$\mathbf{R}_{\mathbf{f}}=0.4$ (Hexane/Ethyl acetate, $5 / 1+1 \%$ Triethylamine)

${ }^{1}$ H NMR (500 MHz, CD $\mathbf{3}$ OD) $\delta 7.52-7.45(\mathrm{~m}, 2 \mathrm{H}), 7.43-7.37(\mathrm{~m}, 2 \mathrm{H}), 7.36-7.25$ (m, 6H), $5.56(\mathrm{~s}, 0.1 \mathrm{H}), 5.53(\mathrm{~s}, 1 \mathrm{H}), 5.18(\mathrm{~d}, J=10.0 \mathrm{~Hz}, 1 \mathrm{H}), 4.88(\mathrm{~d}, J=11.6 \mathrm{~Hz}, 1 \mathrm{H})$, $4.28(\mathrm{dd}, J=10.3,4.9 \mathrm{~Hz}, 1 \mathrm{H}), 4.24-4.19(\mathrm{~m}, 0.2 \mathrm{H}), 4.02$ (at, $J=8.5 \mathrm{~Hz}, 1 \mathrm{H}), 3.76$ (at, $J=10.1 \mathrm{~Hz}, 1 \mathrm{H}$ ), 3.67 (at, $J=9.5 \mathrm{~Hz}, 1 \mathrm{H}$ ), $3.63-3.58$ (m, 1H), 3.12 (s, 3H), 3.03 (s, $0.4 \mathrm{H}), 2.12-2.11(\mathrm{~m}, 0.4 \mathrm{H}), 2.06(\mathrm{~s}, 3 \mathrm{H}), 1.96-1.86(\mathrm{~m}, 2 \mathrm{H}), 1.68-1.45(\mathrm{~m}, 6 \mathrm{H}), 1.37$

$-1.31(\mathrm{~m}, 1 \mathrm{H}), 1.28(\mathrm{~s}, 1 \mathrm{H}), 1.26-1.22(\mathrm{~m}, 1 \mathrm{H})$.

${ }^{13}$ C NMR (125 MHz, CD 3 OD) $\delta 170.5,169.96,137.4,133.8,131.8,131.1,128.6,128.6$, $128.5,128.5,127.6,127.5,127.1,126.0,125.9,101.6,101.5,101.1,87.8,86.5,80.2$, 78.6, 75.1, 72.4, 72.2, 71.2, 70.3, 69.4, 68.1, 68.1, 48.1, 47.9, 47.9, 47.7, 47.6, 47.5, 47.4, $47.2,47.1,47.1,34.2,33.5,33.4,29.3,25.1,22.8,22.7,22.7,22.6,20.0,19.9$.

IR (film, cm ${ }^{-1}$ ) 2932, 2857，1747，1452，1459，1367，1231，1092，1044， 1027. HRMS(ESI) Calc. for $\mathrm{C}_{28} \mathrm{H}_{34} \mathrm{O}_{7} \mathrm{SNa}(\mathrm{M}+\mathrm{Na}): 537.1917$; found : 537.1930; $[\boldsymbol{\alpha}]^{\mathbf{2 6}}{ }_{\mathbf{D}}=-$ $27.8^{\circ}\left(c 0.35, \mathrm{CH}_{2} \mathrm{Cl}_{2}\right)$.

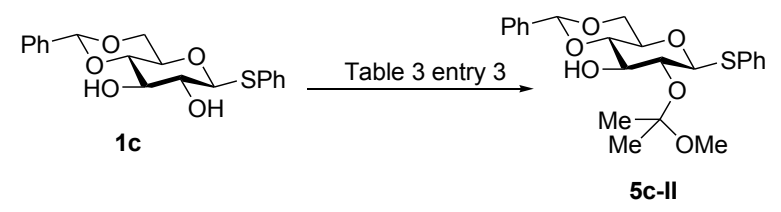

Using diol $1 \mathbf{c}^{5}$ (20 mg, $0.056 \mathrm{mmol}, 1$ equiv.) as the starting material, synthesis of $\mathbf{5 c - I I}$ was accomplished by following general procedure B. This product was purified by flash 
column chromatography $(7 / 1$ Hexanes/Ethyl acetate $+2 \%$ triethylamine $)$ to afford $\mathbf{5 c - I I}$ as a pale yellow oil $(17.3 \mathrm{mg}, 72 \%, \mathrm{C} 2: \mathrm{C} 3=25: 1)$.

${ }^{1}$ H NMR (500 MHz, CD 3 OD) $\delta 7.58-7.41(\mathrm{~m}, 4 \mathrm{H}), 7.41-7.20(\mathrm{~m}, 6 \mathrm{H}), 5.60(\mathrm{~s}, 1 \mathrm{H})$, $5.52(\mathrm{~s}, 0.06 \mathrm{H}), 4.83-4.77(\mathrm{~m}, 1 \mathrm{H}), 4.73(\mathrm{~d}, J=9.1 \mathrm{~Hz}, 0.07 \mathrm{H}), 4.27$ (dd, $J=10.0,5.0$ $\mathrm{Hz}, 1 \mathrm{H}), 3.91(\mathrm{~d}, J=9.0 \mathrm{~Hz}, 0.1 \mathrm{H}), 3.82-3.68(\mathrm{~m}, 3 \mathrm{H}), 3.63-3.55$ (m, 1H), 3.51 (td, $J$ $=9.7,4.9 \mathrm{~Hz}, 1 \mathrm{H}), 3.35(\mathrm{~s}, 3 \mathrm{H}), 3.18(\mathrm{~s}, 0.2 \mathrm{H}), 1.45(\mathrm{~s}, 3 \mathrm{H}), 1.41(\mathrm{~s}, 3 \mathrm{H}), 1.38(\mathrm{~s}, 0.2 \mathrm{H})$, $1.28(\mathrm{~s}, 0.2 \mathrm{H})$.

${ }^{13}$ C NMR (125 MHz, CD $\mathbf{C D}_{3}$ OD) $\delta$ 137.6, 133.8, 131.1, 128.6, 128.5, 127.6, 127.1, 126.1, $101.8,101.5,88.3,87.0,78.0,79.8,75.5,74.3,72.3,70.3,69.6,68.2,49.2,48.7,48.1$, 47.9, 47.8, 47.6, 47.4, 47.2, 47.1, 24.5, 24.03, 23.9, 23.4.

IR (film, $\mathbf{c m}^{-1}$ ) 3374, 2963,2927, 2870, 1579, 1461, 1380, 1275, 1204, 1141, 1123, 1077, 1044, 1022. HRMS(ESI) Calc. for $\mathrm{C}_{23} \mathrm{H}_{28} \mathrm{O}_{6} \mathrm{SNa}(\mathrm{M}+\mathrm{Na}): 455.1499$; found : $455.1511 ;[\boldsymbol{\alpha}]^{26}{ }_{\mathbf{D}}=-25.9^{\circ}\left(c 0.34, \mathrm{CH}_{2} \mathrm{Cl}_{2}\right)$.

Determination of the $\mathrm{C} 2 / \mathrm{C} 3$ ratios was performed by acetylating the crude reaction mixture and analyzing the COSY NMR spectrum of the acetylated $\mathbf{5 c - I I}$.

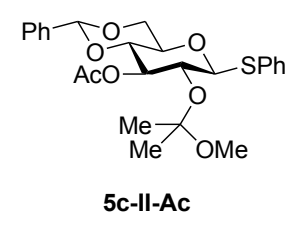

$\mathbf{R}_{\mathbf{f}}=0.3$ (Hexane/Ethyl acetate, $7 / 1+1 \%$ Triethylamine)

${ }^{1}$ H NMR (500 MHz, CD 3 OD) $\delta 7.54-7.45(\mathrm{~m}, 2 \mathrm{H}), 7.44-7.37$ (m, 2H), $7.36-7.22$ (m, 6H), 5.55 (s, 1H), 5.19 (at, $J=10.0 \mathrm{~Hz}, 1 \mathrm{H}), 4.29(\mathrm{dd}, J=10.3,4.9 \mathrm{~Hz}, 1 \mathrm{H}), 3.96$ (t, $J=8.6 \mathrm{~Hz}, 1 \mathrm{H}$ ), 3.77 (at, $J=10.1 \mathrm{~Hz}, 1 \mathrm{H}$ ), 3.68 (at, $J=9.5 \mathrm{~Hz}, 1 \mathrm{H}$ ), $3.65-3.55$ (m, 1H), 3.17 (s, 3H), 3.04 (s, 0.3H), 2.12 (s, 0.4H), 2.06 (s, 3H), 1.40 (s, 3H), 1.35 (s, 3H).

${ }^{13}$ C NMR (125 MHz, CD $\mathbf{C D}_{3}$ OD) $\delta$ 170.6, 137.4, 133.7, 131.2, 128.6, 128.5, 127.6, 127.1, 125.9, 101.8, 101.1, 87.9, 78.5, 75.1, 72.7, 69.5, 68.1, 48.4, 48.1, 47.9, 47.7, 47.6, 47.4, $47.2,47.1,24.5,24.0,19.9$.

IR (film, $\mathbf{c m}^{-1}$ ) 2989, 2914, 2848, 1748, 1456, 1369, 1233, 1088, 1058, 1029, 1001. HRMS(ESI) Calc. for $\mathrm{C}_{25} \mathrm{H}_{30} \mathrm{O}_{7} \mathrm{SNa}(\mathrm{M}+\mathrm{Na}): 497.1604$; found : 497.1612; $[\boldsymbol{\alpha}]^{26}{ }_{\mathbf{D}}=-$ $10.0^{\circ}\left(c 0.30, \mathrm{CH}_{2} \mathrm{Cl}_{2}\right)$. 


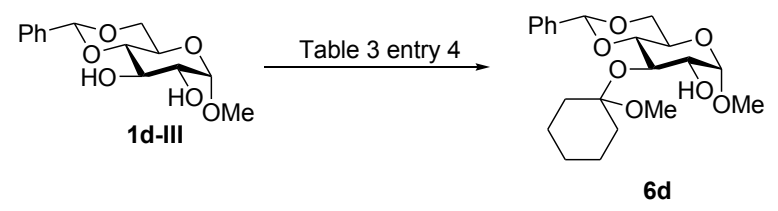

Using diol 1d-III (20 mg, $0.071 \mathrm{mmol}, 1$ equiv.) as the starting material, synthesis of $\mathbf{6 d}$ was accomplished by following general procedure A. This product was purified by flash column chromatography ( $3 / 1$ Hexanes/Ethyl acetate $+1 \%$ triethylamine) to afford $\mathbf{6 d}$ as a pale yellow oil $(22.1 \mathrm{mg}, 79 \%, \mathrm{C} 2: \mathrm{C} 3=1: 8.8)$.

${ }^{1}$ H NMR (500 MHz, CD $\mathbf{3}$ OD) $\delta 7.89(\mathrm{~d}, J=8.1 \mathrm{~Hz}, 0.1 \mathrm{H}), 7.77(\mathrm{~s}, 0.1 \mathrm{H}), 7.51-7.44$ (m, 2H), 7.39 (d, $J=7.9 \mathrm{~Hz}, 0.2 \mathrm{H}), 7.37-7.31(\mathrm{~m}, 3 \mathrm{H}), 7.25(\mathrm{~d}, J=1.9 \mathrm{~Hz}, 0.1 \mathrm{H}), 7.22$ - $7.18(\mathrm{~m}, 0.2 \mathrm{H}), 7.15(\mathrm{~d}, J=1.9 \mathrm{~Hz}, 0.1 \mathrm{H}), 7.10(\mathrm{~d}, J=8.5 \mathrm{~Hz}, 0.2 \mathrm{H}), 5.57(\mathrm{~s}, 0.1 \mathrm{H})$, $5.54(\mathrm{~s}, 1 \mathrm{H}), 4.78(\mathrm{~d}, J=3.6 \mathrm{~Hz}, 0.1 \mathrm{H}), 4.74(\mathrm{~d}, J=3.8 \mathrm{~Hz}, 1 \mathrm{H}), 4.26-4.17(\mathrm{~m}, 1 \mathrm{H})$, 4.07 (at, $J=9.1 \mathrm{~Hz}, 1 \mathrm{H}), 3.83$ (d, $J=8.1 \mathrm{~Hz}, 0.1 \mathrm{H}), 3.79-3.69$ (m, 3H), 3.52 (dd, $J=$ 9.1, $3.8 \mathrm{~Hz}, 1 \mathrm{H}), 3.48(\mathrm{td}, J=9.2,3.0 \mathrm{~Hz}, 1 \mathrm{H}), 3.43$ (s, 3H), $3.40(\mathrm{~s}, 0.3 \mathrm{H}), 3.26(\mathrm{~s}$, $0.3 \mathrm{H}$ ), $3.17(\mathrm{~s}, 3 \mathrm{H}), 3.05$ (dd, $J=14.6,7.6 \mathrm{~Hz}, 0.3 \mathrm{H}), 2.14-2.05$ (m, $0.5 \mathrm{H}), 1.99$ (at, $J=$ $8.4 \mathrm{~Hz}, 1 \mathrm{H}), 1.85-1.77(\mathrm{~m}, 2 \mathrm{H}), 1.73-1.66(\mathrm{~m}, 2 \mathrm{H}), 1.62-1.55(\mathrm{~m}, 2 \mathrm{H}), 1.48-1.39$ (m, 2H), $1.36-1.31(\mathrm{~m}, 1 \mathrm{H}), 1.27-1.23(\mathrm{~m}, 1 \mathrm{H}), 1.20-1.15(\mathrm{~m}, 1 \mathrm{H})$.

${ }^{13}$ C NMR (125 MHz, CD $_{3}$ OD) $\delta 137.8,128.5,127.6,126.1,126.0,101.7,101.6,101.5$, 100.6, 100.3, 81.5, 80.6, 71.9, 70.9, 69.0, 68.7, 68.6, 62.7, 62.1, 54.3, 53.9, 48.0, 47.9, $47.7,47.6,47.6,47.4,47.2,47.1,43.2,36.6,34.0,33.3,33.1,25.1,25.0,22.8,22.6,22.4$, 22.2 .

IR (film, $\mathbf{c m}^{-1}$ ) 3376, 2927, 2853, 1452, 1371, 1275, 1255, 1121, 1078, 1055, 1040, 1022, 996. HRMS(ESI) Calc. for $\mathrm{C}_{21} \mathrm{H}_{30} \mathrm{O}_{7} \mathrm{SNa}(\mathrm{M}+\mathrm{Na})$ : 417.1899; found : 417.1899; $[\boldsymbol{\alpha}]^{26}{ }_{\mathbf{D}}=+25.7^{\circ}\left(c 0.41, \mathrm{CH}_{2} \mathrm{Cl}_{2}\right)$.

Determination of the $\mathrm{C} 2 / \mathrm{C} 3$ ratios was performed by acetylating the crude reaction mixture and analyzing the COSY NMR spectrum of the acetylated $\mathbf{6 d}$.

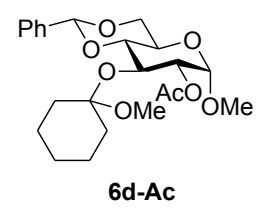

$\mathbf{R}_{\mathbf{f}}=0.5$ (Hexane/Ethyl acetate $4 / 1+1 \%$ Triethylamine) 
${ }^{1}$ H NMR (500 MHz, CD $\mathbf{3}$ OD) $\delta 7.52-7.45(\mathrm{~m}, 2 \mathrm{H}), 7.41(\mathrm{dd}, J=6.8,2.9 \mathrm{~Hz}, 0.16 \mathrm{H})$, $7.39-7.30(\mathrm{~m}, 3 \mathrm{H}), 5.57(\mathrm{~s}, 1 \mathrm{H}), 5.54(\mathrm{~s}, 0,06 \mathrm{H}), 4.86(\mathrm{~d}, J=3.0 \mathrm{~Hz}, 1 \mathrm{H}), 4.73$ (dd, $J=$ 9.6, 3.7 Hz, 1H), 4.29 (at, $J=9.4 \mathrm{~Hz}, 1 \mathrm{H}), 4.25-4.18(\mathrm{~m}, 1 \mathrm{H}), 3.82-3.73(\mathrm{~m}, 2 \mathrm{H}), 3.57$ (at, $J=10.0 \mathrm{~Hz}, 1 \mathrm{H}), 3.43(\mathrm{~s}, 0.3 \mathrm{H}), 3.39(\mathrm{~s}, 3 \mathrm{H}), 3.26-3.23(\mathrm{~m}, 0.2 \mathrm{H}), 3.05(\mathrm{~s}, 3 \mathrm{H})$, $2.09(\mathrm{~s}, 3 \mathrm{H}), 2.04(\mathrm{~s}, 0.2 \mathrm{H}), 1.87-1.75(\mathrm{~m}, 2 \mathrm{H}), 1.60-1.47(\mathrm{~m}, 5 \mathrm{H}), 1.43-1.25(\mathrm{~m}$, $3 \mathrm{H}), 1.23-1.12(\mathrm{~m}, 1 \mathrm{H})$.

${ }^{13}$ C NMR (125 MHz, CD 3 OD) $\delta 170.4,137.7,128.6,127.6,127.6,126.1,126.0,101.8$, 101.7, 101., 101.4, 100.1, 97.8, 81.1, 79.3, 72.9, 70.1, 69.8, 68.5, 67.7, 62.7, 62.2, 54.2, 53.9, 48.1, 48.0, 47.9, 47.9, 47.7, 47.6, 47.5, 47.4, 47.3, 47.2, 47.1, 34.2, 33.7, 33.5, 33.1, $25.1,25.0,22.8,22.7,22.5,22.3,19.7,19.6$.

IR (film, $\mathbf{c m}^{-\mathbf{1}}$ ) 2932, 2853, 1741, 1454, 1369, 1237, 1191, 1154, 1125, 1097, 1049, 998,

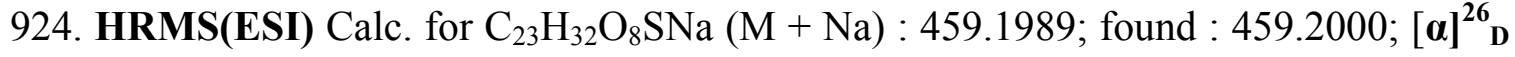
$=+17.7^{\circ}\left(c 0.42, \mathrm{CH}_{2} \mathrm{Cl}_{2}\right)$.

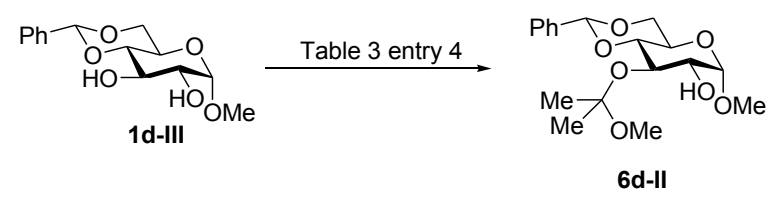

Using diol 1d-III (20 mg, $0.071 \mathrm{mmol}, 1$ equiv.) as the starting material, synthesis of $\mathbf{6 d -}$ II was accomplished by following general procedure B. This product was purified by flash column chromatography (2/1 Hexanes/Ethyl acetate $+1 \%$ triethylamine $)$ to afford 6d-II as a pale yellow oil (17.3 mg, 69\%, C2:C3 = 1:6.3).

${ }^{1}$ H NMR (500 MHz, CD $\mathbf{3}$ OD) $\delta 7.54-7.42(\mathrm{~m}, 3 \mathrm{H}), 7.40-7.28(\mathrm{~m}, 4 \mathrm{H}), 5.57(\mathrm{~s}, 0.2 \mathrm{H})$, $5.52(\mathrm{~s}, 1 \mathrm{H}), 4.77(\mathrm{~d}, J=3.6 \mathrm{~Hz}, 0.2 \mathrm{H}), 4.73(\mathrm{~d}, J=3.6 \mathrm{~Hz}, 1 \mathrm{H}), 4.25-4.16(\mathrm{~m}, 1 \mathrm{H})$, 4.01 (at, $J=9.2 \mathrm{~Hz}, 1 \mathrm{H}), 3.86-3.79(\mathrm{~m}, 0.4 \mathrm{H}), 3.79-3.65(\mathrm{~m}, 3 \mathrm{H}), 3.51(\mathrm{dd}, J=9.2$, $3.5 \mathrm{~Hz}, 1 \mathrm{H}), 3.49-3.45(\mathrm{~m}, 1 \mathrm{H}), 3.43(\mathrm{~s}, 3 \mathrm{H}), 3.41(\mathrm{~s}, 0.7 \mathrm{H}), 3.27(\mathrm{~s}, 0.5 \mathrm{H}), 3.15(\mathrm{~s}, 3 \mathrm{H})$, $1.41(\mathrm{~s}, 0.7 \mathrm{H}), 1.40(\mathrm{~s}, 0.7 \mathrm{H}), 1.38(\mathrm{~s}, 3 \mathrm{H}), 1.36(\mathrm{~s}, 3 \mathrm{H})$.

${ }^{13}$ C NMR (12 MHz, CD $\mathbf{O}$ OD) $\delta 137.8,137.7,128.5,128.5,127.6,127.6,126.3,126.1$, $101.8,101.6,101.5,100.8,100.2,81.5,81.4,80.8,73.0,72.1,71.7,70.6,70.6,69.0,68.7$, 68.6, 62.8, 62.1, 54.4, 54.3, 54.0, 49.0, 48.3, 48.1, 47.9, 47.8, 47.6, 47.4, 47.2, 47.1, 43.2, $36.6,29.2,24.6,24.0,24.0$. 
IR (film, $\mathbf{c m}^{-1}$ ) 3429, 2993, 2932, 2914, 2857, 1456, 1384, 1373, 1207, 1147, 1072, 1075, 992. . HRMS(ESI) Calc. for $\mathrm{C}_{18} \mathrm{H}_{26} \mathrm{O}_{7} \mathrm{SNa}(\mathrm{M}+\mathrm{Na})$ : 377.1571; found : $377.1583 ;[\boldsymbol{\alpha}]^{26}{ }_{\mathbf{D}}=+68.6^{\circ}\left(c 0.37, \mathrm{CH}_{2} \mathrm{Cl}_{2}\right)$.

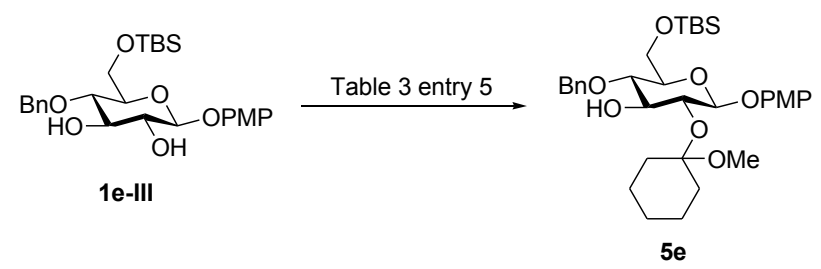

Using diol 1e-III (20 mg, 0.04 mmol, 1 equiv.) as the starting material, synthesis of $\mathbf{5 e}$ was accomplished by following general procedure A. This product was purified by flash column chromatography (5/1 Hexanes/Ethyl acetate $+1 \%$ triethylamine ) to afford $\mathbf{5 e}$ as a pale yellow oil $(19 \mathrm{mg}, 79 \%, \mathrm{C} 2: \mathrm{C} 3=5: 1)$.

${ }^{1}$ H NMR (500 MHz, $\left.\mathbf{C D C l}_{3}\right) \delta 7.41-7.26(\mathrm{~m}, 5 \mathrm{H}), 7.08(\mathrm{~d}, 0.4 \mathrm{H}), 7.00(\mathrm{~d}, 2 \mathrm{H}), 6.80(\mathrm{~d}$, 2H), $5.32-5.28(\mathrm{~m}, 0.3 \mathrm{H}), 5.05$ (s, 1H), $5.02(\mathrm{~d}, J=11.2 \mathrm{~Hz}, 1 \mathrm{H}), 4.85$ (d, $J=11.0 \mathrm{~Hz}$, $0.2 \mathrm{H}), 4.77(\mathrm{~d}, J=7.5 \mathrm{~Hz}, 1 \mathrm{H}), 4.68(\mathrm{~d}, J=11.2 \mathrm{~Hz}, 1 \mathrm{H}), 4.65(\mathrm{~d}, J=11.0 \mathrm{~Hz}, 0.2 \mathrm{H})$, 3.98 (at, $J=8.5 \mathrm{~Hz}, 0.2 \mathrm{H}), 3.94-3.88(\mathrm{~m}, 1 \mathrm{H}), 3.86-3.79$ (m, 1H), 3.77 (s, 3H), $3.75-$ 3.67 (m, 2H), $3.58-3.41$ (m, 2H), 3.39 (s, 0.4H), 3.36 (s, 3H), $2.05-1.98(\mathrm{~m}, 1 \mathrm{H}), 1.86$ $-1.70(\mathrm{~m}, 3 \mathrm{H}), 1.68-1.48(\mathrm{~m}, 6 \mathrm{H}), 1.45-1.29(\mathrm{~m}, 2 \mathrm{H}), 1.26(\mathrm{~s}, 2 \mathrm{H}), 0.89(\mathrm{~s}, 9 \mathrm{H}), 0.06$ (s, $0.4 \mathrm{H}), 0.05(\mathrm{~s}, 3 \mathrm{H}), 0.02(\mathrm{~s}, 3 \mathrm{H})$.

${ }^{13} \mathbf{C}$ NMR (125 MHz, $\left.\mathbf{C D C l}_{3}\right) \delta 155.2,155.0,152.0,151.6,138.6,138.3,128.4,128.3$, 128.1, 127.8, 127.6, 119.1, 118.2, 114.4, 114.3, 102.3, 101.6, 101.5, 101.2, 78.9, 77.6, $77.4,77.2,77.0,76.7,76.1,76.1,75.9,75.4,75.1,74.8,73.3,62.7,62.4,55.6,55.6,48.2$, $48.1,33.8,33.6,33.5,32.9,29.7,25.9,25.3,25.3,22.8,22.8,21.9,18.3,-5.2,-5.3,-5.35$, -5.41 .

IR (film, $\mathbf{c m}^{-1}$ ) 3380, 2927, 2852, 1507, 1463, 1250, 1226, 1154, 1093, 1038, 919. HRMS(ESI) Calc. for $\mathrm{C}_{33} \mathrm{H}_{50} \mathrm{O}_{8} \mathrm{SiNa}(\mathrm{M}+\mathrm{Na}): 625.3167$; found : $625.3182 ;[\boldsymbol{\alpha}]^{\mathbf{2 6}}{ }_{\mathbf{D}}=-$ $5.9^{\circ}\left(c 0.24, \mathrm{CH}_{2} \mathrm{Cl}_{2}\right)$.

Determination of the $\mathrm{C} 2 / \mathrm{C} 3$ ratios was performed by acetylating the crude reaction mixture and analyzing the COSY NMR spectrum of the acetylated $\mathbf{5 e}$. 


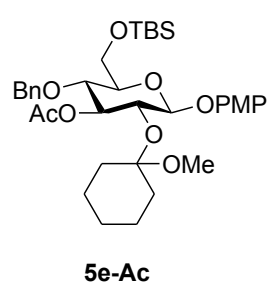

$\mathbf{R}_{\mathbf{f}}=0.4$ (Hexane/Ethyl acetate $5 / 1+1 \%$ Triethylamine)

${ }^{1}$ H NMR (500 MHz, $\left.\mathbf{C D C l}_{3}\right) \delta 7.37$ - 7.31 (m, 3H), 7.31 - 7.27 (m, 3H), 7.02 - 6.97 (m, 2H), $6.94(\mathrm{~d}, J=10.0 \mathrm{~Hz}, 0.4 \mathrm{H}), 6.84-6.76(\mathrm{~m}, 3 \mathrm{H}), 5.21(\mathrm{t}, J=8.5 \mathrm{~Hz}, 1 \mathrm{H}), 5.05$ (at, $J$ $=7.5 \mathrm{~Hz}, 0.4 \mathrm{H}), 4.87(\mathrm{~d}, J=6.9 \mathrm{~Hz}, 1 \mathrm{H}), 4.83(\mathrm{dd}, J=9.2,6.4 \mathrm{~Hz}, 0.6 \mathrm{H}), 4.66-4.58$ (m, 2H), 4.15 (at, $J=7.8 \mathrm{~Hz}, 0.4 \mathrm{H}), 4.00(\mathrm{dd}, J=8.0,7.1 \mathrm{~Hz}, 1 \mathrm{H}), 3.88-3.85(\mathrm{~m}, 0.3 \mathrm{H})$, $3.83(\mathrm{dd}, J=11.4,2.3 \mathrm{~Hz}, 1 \mathrm{H}), 3.78$ (d, $J=4.5 \mathrm{~Hz}, 1 \mathrm{H}), 3.77$ (s, 3H), 3.76 (s, 0.8H), 3.66 (at, $J=10.5 \mathrm{~Hz}, 1 \mathrm{H}), 3.62(\mathrm{~d}, J=8.0 \mathrm{~Hz}, 0.3 \mathrm{H}), 3.51(\mathrm{ddd}, J=9.2,4.4,2.4 \mathrm{~Hz}, 1 \mathrm{H}), 3.16$ $(\mathrm{s}, 0.6 \mathrm{H}), 3.13(\mathrm{~s}, 3 \mathrm{H}), 2.10(\mathrm{~s}, 0.6 \mathrm{H}), 2.01(\mathrm{~s}, 3 \mathrm{H}), 1.92-1.84(\mathrm{~m}, 2 \mathrm{H}), 1.58-1.55(\mathrm{~m}$, 2H), $1.54-1.50(\mathrm{~m}, 2 \mathrm{H}), 1.46-1.40(\mathrm{~m}, 2 \mathrm{H}), 1.27-1.23(\mathrm{~m}, 5 \mathrm{H}), 0.89(\mathrm{~s}, 9 \mathrm{H}), 0.88(\mathrm{~s}$, 2H), $0.04(\mathrm{~s}, 0.7 \mathrm{H}), 0.03(\mathrm{~s}, 3 \mathrm{H}), 0.02(\mathrm{~s}, 0.7 \mathrm{H}), 0.01(\mathrm{~s}, 3 \mathrm{H})$.

${ }^{13}$ C NMR (125 MHz, $\left.\mathbf{C D C l}_{3}\right) \delta 170.0,169.7,155.2,155.0,151.6,151.4,138.2,137.9$, $128.4,128.0,127.8,127.8,118.5,118.3,114.4,101.8,101.6,101.5,100.6,77.2,77.2$, 77.0, 76.7, 76.7, 76.5, 76.2, 76.0, 75.7, 74.5, 74.2, 73.9, 73.5, 71.7, 62.4, 62.2, 55.6, 48.4, 48.2 , 34.4, 34.1, 34.0, 33.9, 31.9, 29.7, 29.4, 25.9, 25.4, 23.1, 23.0, 22.7, 21.4, 21.3, 18.3, $14.1,-5.17,-5.2,-5.4,-5.5$

IR (film, $\mathbf{c m}^{-1}$ ) 2927, 2853, 1750, 1507, 1463, 1364, 1222, 1154, 1088, 1060, 924, 836. HRMS(ESI) Calc. for $\mathrm{C}_{35} \mathrm{H}_{52} \mathrm{O}_{8} \mathrm{SiNa}(\mathrm{M}+\mathrm{Na}): 667.3273$; found : 667.3283 ; $[\boldsymbol{\alpha}]^{\mathbf{2 6}}{ }_{\mathbf{D}}=-$ $13.9^{\circ}\left(c 0.32, \mathrm{CH}_{2} \mathrm{Cl}_{2}\right)$.

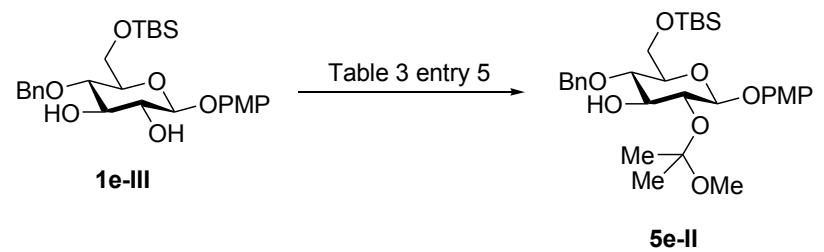

Using diol 1e-III (20 mg, 0.04 mmol, 1 equiv.) as the starting material, synthesis of 5e-II was accomplished by following general procedure B. This product was purified by flash column chromatography $(5 / 1$ Hexanes/Ethyl acetate $+1 \%$ triethylamine $)$ to afford 5e-II as pale yellow oil $(20.2 \mathrm{mg}, 88 \%, \mathrm{C} 2: \mathrm{C} 3=7: 1)$. 
${ }^{1}$ H NMR (500 MHz, CD $\mathbf{3}$ OD) $\delta 7.37$ (d, $\left.J=1.5 \mathrm{~Hz}, 1 \mathrm{H}\right), 7.36-7.35$ (m, 2H), 7.34 $7.32(\mathrm{~m}, 2 \mathrm{H}), 7.31-7.31(\mathrm{~m}, 0.5 \mathrm{H}), 7.30$ - $7.25(\mathrm{~m}, 1 \mathrm{H}), 7.05-7.01(\mathrm{~m}, 1 \mathrm{H}), 6.99$ $6.95(\mathrm{~m}, 2 \mathrm{H}), 6.82-6.81(\mathrm{~m}, 1 \mathrm{H}), 6.80-6.79(\mathrm{~m}, 1 \mathrm{H}), 4.95(\mathrm{~d}, J=11.2 \mathrm{~Hz}, 1 \mathrm{H}), 4.82-$ $4.78(\mathrm{~m}, 1 \mathrm{H}), 4.72(\mathrm{~d}, J=7.8 \mathrm{~Hz}, 0.3 \mathrm{H}), 4.69-4.64(\mathrm{~m}, 1 \mathrm{H}), 3.94$ (at, $J=8.6 \mathrm{~Hz}, 0.2 \mathrm{H})$, $3.87-3.84(\mathrm{~m}, 1 \mathrm{H}), 3.78-3.74(\mathrm{~m}, 1 \mathrm{H}), 3.74-3.72(\mathrm{~m}, 6 \mathrm{H}), 3.72-3.69(\mathrm{~m}, 1 \mathrm{H}), 3.69$ - $3.64(\mathrm{~m}, 1 \mathrm{H}), 3.62(\mathrm{~d}, J=9.1 \mathrm{~Hz}, 0.4 \mathrm{H}), 3.50-3.46(\mathrm{~m}, 1 \mathrm{H}), 3.45-3.43(\mathrm{~m}, 0.4 \mathrm{H})$, $3.43-3.39(\mathrm{~m}, 1 \mathrm{H}), 3.36(\mathrm{~s}, 0.5 \mathrm{H}), 3.32(\mathrm{~s}, 3 \mathrm{H}), 1.51(\mathrm{~s}, 3 \mathrm{H}), 1.47(\mathrm{~s}, 1 \mathrm{H}), 1.46(\mathrm{~s}, 3 \mathrm{H})$, 0.89 (s, 3H), $0.88(\mathrm{~d}, J=2.8 \mathrm{~Hz}, 9 \mathrm{H}), 0.04(\mathrm{~s}, 0.5 \mathrm{H}), 0.04(\mathrm{~s}, 0.5 \mathrm{H}), 0.03(\mathrm{~s}, 3 \mathrm{H}),-0.01$ (s, $3 \mathrm{H})$.

${ }^{13}$ C NMR (125 MHz, CD 3 OD) $\delta$ 155.2, 155.1, 151.7, 151.6, 138.6, 138.3, 128.0, 127.9, $127.7,127.7,127.5,127.3,118.0,117.9,117.5,114.0,113.9,101.9,101.8,100.7,78.7$, 77.7, 77.6, 76.9, 76.8, 76.3, 75.8, 75.8, 75.6, 75.3, 74.6, 74.4, 74.4, 73.9, 73.1, 62.3, 54.6, 49.1, 48.1, 47.9, 47.8, 47.6, 47.4, 47.2, 47.1, 25.0, 24.5, 23.8, 17.8, -6.4, -6.6.

IR (film, $\mathbf{c m}^{-1}$ ) 3407, 2931, 2853, 1509, 1463, 1384, 1370, 1228, 1066, 834. HRMS(ESI) Calc. for $\mathrm{C}_{30} \mathrm{H}_{46} \mathrm{O}_{8} \mathrm{SiNa}(\mathrm{M}+\mathrm{Na}): 585.2854$; found : $585.2850 ;[\boldsymbol{\alpha}]^{\mathbf{2 6}}{ }_{\mathbf{D}}=-$ $13.7^{\circ}\left(c 0.09, \mathrm{CH}_{2} \mathrm{Cl}_{2}\right)$.

Determination of the $\mathrm{C} 2 / \mathrm{C} 3$ ratios was performed by acetylating the crude reaction mixture and analyzing the COSY NMR spectrum of the acetylated 5e-II.

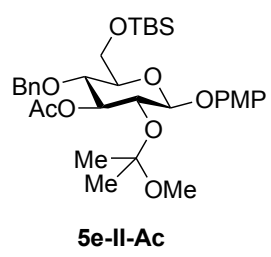

$\mathbf{R}_{\mathbf{f}}=0.3$ (Hexane/Ethyl acetate, $4.5 / 1+2 \%$ Triethylamine)

${ }^{1}$ H NMR (500 MHz, CD $\mathbf{3}$ OD) $\delta 7.36-7.31(\mathrm{~m}, 2 \mathrm{H}), 7.31-7.25(\mathrm{~m}, 3 \mathrm{H}), 7.04-7.01$ $(\mathrm{m}, 0.2 \mathrm{H}), 7.00-6.96(\mathrm{~m}, 2 \mathrm{H}), 6.94-6.91(\mathrm{~m}, 0.3 \mathrm{H}), 6.85-6.78(\mathrm{~m}, 2 \mathrm{H}), 5.16$ (at, $J=$ $10.0 \mathrm{~Hz}, 1 \mathrm{H}), 4.95-4.89(\mathrm{~m}, 1 \mathrm{H}), 4.70-4.57$ (m, 2H), $3.90-3.85$ (m, 2H), 3.81 (dd, $J$ $=11.7,4.3 \mathrm{~Hz}, 1 \mathrm{H}), 3.74(\mathrm{~s}, 3 \mathrm{H}), 3.73(\mathrm{~s}, 1 \mathrm{H}), 3.69$ (at, $J=9.4 \mathrm{~Hz}, 1 \mathrm{H}), 3.58(\mathrm{~d}, J=9.4$ $\mathrm{Hz}, 0.2 \mathrm{H}), 3.52$ (ddd, $J=9.6,4.2,2.0 \mathrm{~Hz}, 1 \mathrm{H}), 3.19$ (s, 1H), 3.17 (s, 3H), 2.09 (s, 0.3H), $2.00(\mathrm{~s}, 3 \mathrm{H}), 1.43(\mathrm{~s}, 44 \mathrm{H}), 1.40(\mathrm{~s}, 3 \mathrm{H}), 1.38(\mathrm{~s}, 3 \mathrm{H}), 1.28(\mathrm{~s}, 0.4 \mathrm{H}), 0.91(\mathrm{~s}, 1 \mathrm{H}), 0.90(\mathrm{~s}$, 9H), 0.06 (s, 0.4H), 0.05 (s, 3H), $0.02(\mathrm{~s}, 0.4 \mathrm{H}), 0.01(\mathrm{~s}, 3 \mathrm{H})$. 
${ }^{13}$ C NMR (125 MHz, CD 3 OD ) $\delta$ 170.6, 155.2, 151.2, 138.1, 128.0, 127.7, 127.5, 127.4, $118.1,117.8,117.4,114.1,101.7,100.8,100.2,77.0,76.2,75.9,75.4,75.4,74.0,73.8$, 73.0, 62.1, 61.9, 54.6, 48.7, 48.1, 48.0, 47.9, 47.9, 47.7, 47.6, 47.4, 47.2, 47.1, 25.0, 24.9, 24.7, 24.2, 24.1, 20.1, 19.9, 17.8, -6.4, -6.7.

IR (film, $\mathbf{~ c m}^{-1}$ ) 2923, 1748, 1505, 1459, 1367, 1218, 1057. HRMS(ESI) Calc. for $\mathrm{C}_{32} \mathrm{H}_{48} \mathrm{O}_{9} \mathrm{SiNa}(\mathrm{M}+\mathrm{Na}): 627.2960$; found : 627.2950; $[\boldsymbol{\alpha}]^{26}{ }_{\mathbf{D}}=-5.3^{\circ}\left(c 0.39, \mathrm{CH}_{2} \mathrm{Cl}_{2}\right)$.

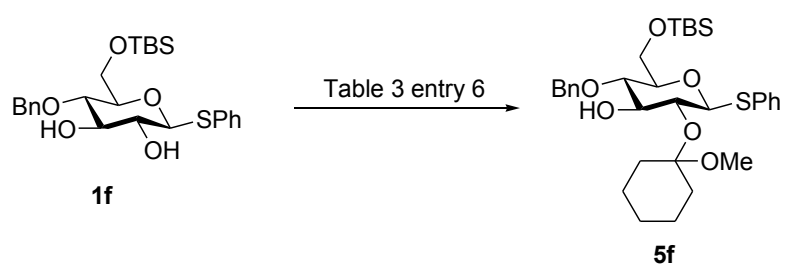

Using diol $\mathbf{1 f}$ (20 mg, $0.04 \mathrm{mmol}, 1$ equiv.) as the starting material, preparation of $\mathbf{5 f}$ was accomplished by following general procedure A. This product was purified by flash column chromatography $(9 / 1$ Hexanes/Ethyl acetate $+1 \%$ triethylamine $)$ to afford $\mathbf{5 f}$ as a pale yellow oil (16.2 mg, 78\%, C2:C3 = 7:1).

${ }^{1} \mathbf{H}$ NMR (500 MHz, $\left.\mathbf{C}_{6} \mathbf{D}_{6}\right) \delta 7.77(\mathrm{~d}, J=5.0 \mathrm{~Hz}, 0.5 \mathrm{H}), 7.61(\mathrm{dd}, J=12.7,5.4 \mathrm{~Hz}, 2 \mathrm{H})$, $7.43(\mathrm{~d}, J=7.2 \mathrm{~Hz}, 2 \mathrm{H}), 7.32(\mathrm{~d}, J=7.3 \mathrm{~Hz}, 0.5 \mathrm{H}), 7.19-7.13(\mathrm{~m}, 2 \mathrm{H}), 7.08-7.02$ (m, 4H), $7.01-6.91(\mathrm{~m}, 2 \mathrm{H}), 5.38(\mathrm{~s}, 1 \mathrm{H}), 5.33$ (d, $J=10.0 \mathrm{~Hz}, 1 \mathrm{H}), 4.87$ (d, $J=11.4 \mathrm{~Hz}$, $1 \mathrm{H}), 4.74-4.66(\mathrm{~m}, 1 \mathrm{H}), 3.94-3.86(\mathrm{~m}, 2 \mathrm{H}), 3.86-3.78(\mathrm{~m}, 3 \mathrm{H}), 3.78-3.70(\mathrm{~m}, 2 \mathrm{H})$, 3.62 (d, $J=9.4 \mathrm{~Hz}, 0.3 \mathrm{H}), 3.54$ (at, $J=8.4 \mathrm{~Hz}, 0.3 \mathrm{H}), 3.27$ (ddd, $J=9.7,4.7,1.6 \mathrm{~Hz}$, $1 \mathrm{H}), 3.06(\mathrm{~d}, J=9.8 \mathrm{~Hz}, 0.3 \mathrm{H}), 2.93(\mathrm{~s}, 3 \mathrm{H}), 2.86(\mathrm{~s}, 0.5 \mathrm{H}), 1.74-1.55(\mathrm{~m}, 5 \mathrm{H}), 1.43$ (d, $J=5.1 \mathrm{~Hz}, 1 \mathrm{H}), 1.36-1.28(\mathrm{~m}, 4 \mathrm{H}), 1.18(\mathrm{~d}, J=8.9 \mathrm{~Hz}, 2 \mathrm{H}), 0.96$ (s, 9H), $0.89-0.83$ $(\mathrm{m}, 1 \mathrm{H}), 0.39(\mathrm{~s}, 0.7 \mathrm{H}), 0.14(\mathrm{~s}, 0.5 \mathrm{H}), 0.11(\mathrm{~s}, 3 \mathrm{H}), 0.07(\mathrm{~s}, 0.5 \mathrm{H}), 0.06(\mathrm{~s}, 3 \mathrm{H})$.

${ }^{13}$ C NMR (126 MHz, C $\left.\mathbf{6}_{6}\right) \delta 139.3,139.1,135.4,134.6,132.8,131.0,128.7,128.5$, $128.2,128.1,128.0,127.9,127.8,127.7,127.5,127.3,127.1,127.0,126.6,101.3,101.2$, 87.6, 86.6, 80.6, 79.9, 79.2, 77.0, 75.9, 74.9, 74.7, 74.6, 72.58, 62.8, 62.4, 47.3, 33.4, 33.3, 33.1, 29.8, 25.9, 25.8, 25.1, 25.0, 22.6, 22.5, 22.4, 22.2, 18.3, -5.1, -5.3, -5.5, -5.5.

IR (film, $\mathbf{~ c m}^{-1}$ ) 3380, 2928, 2855, 1462, 1361, 1253, 1153, 1091, 1042, 1025, 919, 906. HRMS(ESI) Calc. for $\mathrm{C}_{32} \mathrm{H}_{48} \mathrm{O}_{6} \mathrm{SSiNa}(\mathrm{M}+\mathrm{Na}): 611.2833$; found : 611.2840; $[\boldsymbol{\alpha}]^{\mathbf{2 6}}{ }_{\mathbf{D}}=$ $-2.73^{\circ}\left(c 0.07, \mathrm{CH}_{2} \mathrm{Cl}_{2}\right)$. 
Determination of the $\mathrm{C} 2 / \mathrm{C} 3$ ratios was performed by acetylating the crude reaction mixture and analyzing the COSY NMR spectrum of the acetylated $\mathbf{5 f}$.

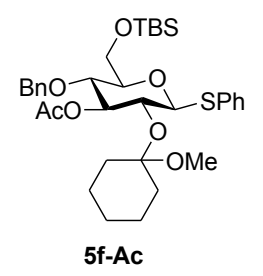

$\mathbf{R}_{\mathbf{f}}=0.3$ (Hexane/Ethyl acetate, $9 / 1+2 \%$ Triethylamine)

${ }^{1}$ H NMR (500 MHz, $\left.\mathbf{C}_{6} \mathbf{D}_{\mathbf{6}}\right) \delta 7.60(\mathrm{dd}, J=8.1,7.0 \mathrm{~Hz}, 2 \mathrm{H}), 7.37(\mathrm{~d}, J=7.1 \mathrm{~Hz}, 2 \mathrm{H})$, $7.16-7.13$ (m, 3H), $7.08-7.03(\mathrm{~m}, 3 \mathrm{H}), 6.97-6.93(\mathrm{~m}, 1 \mathrm{H}), 5.42$ (at, $J=8.2 \mathrm{~Hz}, 1 \mathrm{H}$ ), 4.69 (d, $J=8.7 \mathrm{~Hz}, 1 \mathrm{H}), 4.65$ (s, 2H), 4.06 (at, $J=8.3 \mathrm{~Hz}, 1 \mathrm{H}), 3.76$ (at, $J=9.1 \mathrm{~Hz}, 1 \mathrm{H}$ ), $3.71(\mathrm{dd}, J=11.5,3.5 \mathrm{~Hz}, 1 \mathrm{H}), 3.64$ (dd, $J=11.5,1.8 \mathrm{~Hz}, 1 \mathrm{H}), 3.16$ (s, $3 \mathrm{H}), 3.10$ (dt, $J=$ 5.1, $4.5 \mathrm{~Hz}, 1 \mathrm{H}), 3.07$ (s, 0.2H), $1.99-1.93(\mathrm{~m}, 1 \mathrm{H}), 1.91$ (s, 0.3H), $1.80(\mathrm{~s}, 3 \mathrm{H}), 1.63-$ $1.56(\mathrm{~m}, 1 \mathrm{H}), 1.51-1.38(\mathrm{~m}, 4 \mathrm{H}), 1.34-1.24(\mathrm{~m}, 4 \mathrm{H}), 1.09-1.03(\mathrm{~m}, 1 \mathrm{H}), 0.95(\mathrm{~s}, 9 \mathrm{H})$, $0.38(\mathrm{~s}, 2 \mathrm{H}), 0.11(\mathrm{~s}, 3 \mathrm{H}), 0.09-0.07(\mathrm{~m}, 1 \mathrm{H}), 0.06--0.01(\mathrm{~m}, 3 \mathrm{H})$.

${ }^{13} \mathbf{C}$ NMR (125 MHz, $\left.\mathbf{C}_{6} \mathbf{D}_{6}\right) \delta 169.1,138.6,135.2,131.5,131.3,128.7,128.3,127.9$, 127.8, 127.7, 127.7, 127.6, 127.5, 126.8, 101.6, 87.67, 79.1, 77.0, 75.9, 73.8, 71.4, 62.1, $47.9,34.0,33.8,29.8,25.8,25.4,23.2,23.0,20.9,18.2,-5.2,-5.6$.

IR (film, $\mathbf{~ c m}^{-1}$ ) 2927, 2852, 1750, 1471, 1459, 1362, 1231, 1088, 1048, 921, 908, 836. . HRMS(ESI) Calc. for $\mathrm{C}_{34} \mathrm{H}_{50} \mathrm{O}_{7} \mathrm{SSiNa}(\mathrm{M}+\mathrm{Na}): 653.2939$; found : $653.2948 ;[\boldsymbol{\alpha}]^{\mathbf{2 6}} \mathbf{\mathbf { D }}=$ $-3.32^{\circ}\left(c 0.15, \mathrm{CH}_{2} \mathrm{Cl}_{2}\right)$.

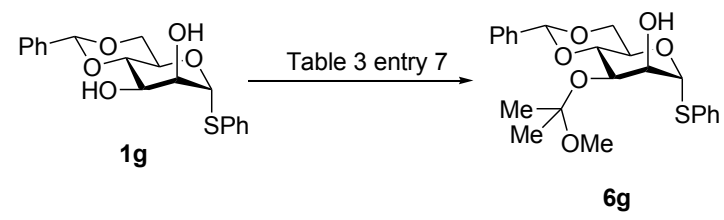

Using diol $\mathbf{1 g}$ (20 $\mathrm{mg}, 0.04 \mathrm{mmol}, 1$ equiv.) as the starting material, synthesis of $\mathbf{6 g}$ was accomplished by following general procedure B. This product was purified by flash column chromatography (3/1 Hexanes/Ethyl acetate $+1 \%$ triethylamine) to afford $\mathbf{6 g}$ as a pale yellow oil (16.2 mg, 78\%, C2:C3 = 1:12.6). 
${ }^{1}$ H NMR (500 MHz, CD $\mathbf{3}$ OD) $\delta 7.54-7.43(\mathrm{~m}, 4 \mathrm{H}), 7.39-7.25(\mathrm{~m}, 6 \mathrm{H}), 5.58(\mathrm{~s}, 1 \mathrm{H})$, $5.48(\mathrm{~s}, 1 \mathrm{H}), 4.29$ (td, $J=9.8,4.8 \mathrm{~Hz}, 1 \mathrm{H}), 4.22(\mathrm{~d}, J=3.0 \mathrm{~Hz}, 1 \mathrm{H}), 4.19$ (dd, $J=10.0$, $3.1 \mathrm{~Hz}, 1 \mathrm{H}), 4.11(\mathrm{dd}, J=10.2,4.8 \mathrm{~Hz}, 1 \mathrm{H}), 4.05$ (at, $J=9.7 \mathrm{~Hz}, 1 \mathrm{H}), 3.82$ (at, $J=10.3$ $\mathrm{Hz}, 1 \mathrm{H}), 3.21$ (s, 3H), 1.40 (d, $J=12.0 \mathrm{~Hz}, 3 \mathrm{H}), 1.37$ (s, 3H).

${ }^{13}$ C NMR (125 MHz, CD 3 OD) $\delta$ 137.9, 133.7, 131.6, 128.8, 128.5, 127.6, 127.3, 126.0, $102.0,101.5,89.5,77.3,72.5,68.1,65.7,48.6,48.1,47.1,24.5,24.2$.

IR (film, $\mathbf{c m}^{-1}$ ) 3433, 2985, 2923, 2853, 1456, 1373, 1211, 1169, 1095, 1031, 970. . HRMS(ESI) Calc. for $\mathrm{C}_{23} \mathrm{H}_{28} \mathrm{O}_{6} \mathrm{SNa}(\mathrm{M}+\mathrm{Na}): 455.1499$; found : 455.1513; $[\boldsymbol{\alpha}]^{\mathbf{2 6}}{ }_{\mathbf{D}}=$ $+48.9^{\circ}\left(c 0.51, \mathrm{CH}_{2} \mathrm{Cl}_{2}\right)$.

Determination of the $\mathrm{C} 2 / \mathrm{C} 3$ ratios was performed by acetylating the crude reaction mixture and analyzing the COSY NMR spectrum of the acetylated $\mathbf{6 g}$.

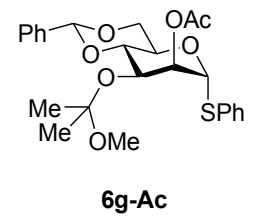

$\mathbf{R}_{\mathbf{f}}=0.35$ (Hexane/Ethyl acetate, $4 / 1+1 \%$ Triethylamine)

${ }^{1}$ H NMR (500 MHz, CD $\mathbf{3}$ OD) $\delta 7.54-7.50(\mathrm{~m}, 2 \mathrm{H}), 7.50-7.45(\mathrm{~m}, 2 \mathrm{H}), 7.39-7.28$ (m, 6H), $5.62(\mathrm{~s}, 1 \mathrm{H}), 5.50-5.46(\mathrm{~m}, 2 \mathrm{H}), 4.37-4.28(\mathrm{~m}, 2 \mathrm{H}), 4.14(\mathrm{dd}, J=10.3,4.9$ Hz, 1H), 4.03 (at, $J=9.8 \mathrm{~Hz}, 1 \mathrm{H}), 3.84$ (at, $J=10.3 \mathrm{~Hz}, 1 \mathrm{H}), 3.13$ (s, 3H), 2.13 (s, 3H), 1.37 (s, 3H), 1.29 (s, 3H).

${ }^{13}$ C NMR (125 MHz, CD $\mathbf{3}$ OD) $\delta$ 170.3, 137.7, 132.9, 131.89, 128.9, 128.6, 127.7, 127.65, 126.0, 102.1, 101.6, 87.0, 77.6, 73.6, 67.9, 66.7, 65.7, 48.7, 48.1, 47.9, 47.7, 47.6, 47.4, 47.2, 47.1, 24.1, 23.9, 19.4 .

IR (film, $\left.\mathbf{c m}^{-\mathbf{1}}\right)$ 2998, 2919, 1744, 1458, 1373, 1233, 1099, 1033. $[\boldsymbol{\alpha}]^{\mathbf{2 6}} \mathbf{D}=+92.1^{\circ}(c$ $\left.0.51, \mathrm{CH}_{2} \mathrm{Cl}_{2}\right)$.

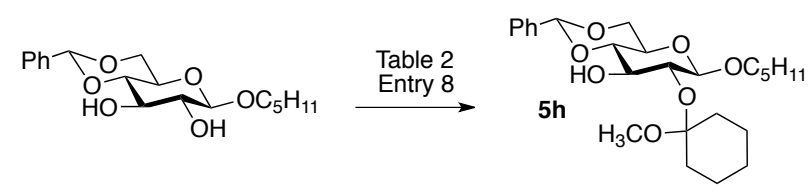


Using the above diol (20 mg, $0.059 \mathrm{mmol}, 1$ equiv.) as the starting material, synthesis of the acetal was accomplished by following general procedure A. This product was purified by flash column chromatography ( $9 / 1$ Hexanes/Ethyl acetate $+2 \%$ triethylamine) to afford the acetal as a pale yellow oil $(21.3 \mathrm{mg}, 80 \%, \mathrm{C} 2: \mathrm{C} 3=>22.1)$.

${ }^{1}$ H NMR (500 MHz, C $\left.\mathbf{C}_{6} \mathbf{D}_{6}\right) \delta 7.67(\mathrm{~d}, J=7.4 \mathrm{~Hz}, 2 \mathrm{H}), 7.14(\mathrm{~d}, J=7.2 \mathrm{~Hz}, 1 \mathrm{H}), 7.09-$ $7.04(\mathrm{~m}, 1 \mathrm{H}), 5.37(\mathrm{~s}, 1 \mathrm{H}), 5.03(\mathrm{~s}, 1 \mathrm{H}), 4.30(\mathrm{~d}, J=5.0 \mathrm{~Hz}, 1 \mathrm{H}), 4.26-4.18(\mathrm{~m}, 1 \mathrm{H})$, $3.90-3.77$ (m, 3H), 3.63 (at, $J=9.1 \mathrm{~Hz}, 1 \mathrm{H}), 3.55$ (at, $J=10.2 \mathrm{~Hz}, 1 \mathrm{H}), 3.37-3.27$ (m, 2H), $2.98(\mathrm{~s}, 3 \mathrm{H}), 1.98(\mathrm{~d}, J=9.3 \mathrm{~Hz}, 1 \mathrm{H}), 1.74-1.62(\mathrm{~m}, 3 \mathrm{H}), 1.59-1.46(\mathrm{~m}, 3 \mathrm{H}), 1.45$ $-1.05(\mathrm{~m}, 9 \mathrm{H}), 0.94-0.87(\mathrm{~m}, 1 \mathrm{H}), 0.83(\mathrm{t}, J=7.2 \mathrm{~Hz}, 3 \mathrm{H})$.

${ }^{13}$ C NMR (125 MHz, $\left.\mathbf{C}_{6} \mathbf{D}_{6}\right) \delta 138.2,128.5,127.9,127.8,127.6,127.5,126.5,102.4$, $101.3,101.3,80.7,76.7,73.6,69.6,68.7,66.0,47.4,33.7,32.9,29.6,28.3,25.4,22.7$, 22.4, 21.7, 13.9 .

IR (film, $\mathbf{c m}^{-1}$ ) 3382, 2936, 2867, 1456, 1368, 1093, 1018.

HRMS(ESI) Calc. for $\mathrm{C}_{25} \mathrm{H}_{38} \mathrm{O}_{7} \mathrm{Na}(\mathrm{M}+\mathrm{Na}): 473.2510$; found : 473.2506; $[\boldsymbol{\alpha}]^{\mathbf{2 6}}{ }_{\mathbf{D}}=-$ $27.8^{\circ}\left(c 0.56, \mathrm{CH}_{2} \mathrm{Cl}_{2}\right)$.

Determination of $\mathrm{C} 2 / \mathrm{C} 3$ ratios was done using COSY experiments of acetylated product.

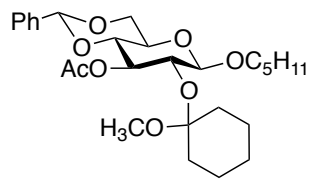

${ }^{1}$ H NMR (400 MHz, C $\left.6 \mathbf{D}_{6}\right) \delta 7.57(\mathrm{~d}, J=7.6 \mathrm{~Hz}, 2 \mathrm{H}), 7.07-6.97(\mathrm{~m}, 2 \mathrm{H}), 5.50(\mathrm{dd}, J=$ 9.3, $7.7 \mathrm{~Hz}, 1 \mathrm{H}), 5.24$ (s, 1H), 4.31 (d, $J=6.5 \mathrm{~Hz}, 1 \mathrm{H}), 4.13(\mathrm{dd}, J=10.0,4.6 \mathrm{~Hz}, 1 \mathrm{H})$, $4.03(\mathrm{t}, J=7.0 \mathrm{~Hz}, 1 \mathrm{H}), 3.77-3.68(\mathrm{~m}, 1 \mathrm{H}), 3.58(\mathrm{t}, J=9.4 \mathrm{~Hz}, 1 \mathrm{H}), 3.44(\mathrm{t}, J=10.0$ $\mathrm{Hz}, 1 \mathrm{H}), 3.40-3.32(\mathrm{~m}, 1 \mathrm{H}), 3.31-3.23(\mathrm{~m}, 1 \mathrm{H}), 3.16(\mathrm{~s}, 3 \mathrm{H}), 1.92-1.81(\mathrm{~m}, 2 \mathrm{H}), 1.79$ $(\mathrm{s}, 3 \mathrm{H}), 1.75-1.64(\mathrm{~m}, 1 \mathrm{H}), 1.64-1.42(\mathrm{~m}, 6 \mathrm{H}), 1.40-1.32(\mathrm{~m}, 2 \mathrm{H}), 1.31-1.13(\mathrm{~m}$, $5 \mathrm{H}), 0.89$ (t, $J=7.2 \mathrm{~Hz}, 1 \mathrm{H}), 0.81(\mathrm{t}, J=7.1 \mathrm{~Hz}, 3 \mathrm{H})$.

${ }^{13}$ C NMR (100 MHz, $\left.\mathbf{C}_{6} \mathbf{D}_{6}\right) \delta$ 169.0, 137.8, 128.6, 127.9, 127.8, 127.6, 127.5, 127.4, $126.3,102.9,101.5,101.3,79.2,73.9,72.5,69.4,68.9,65.3,47.9,34.2,34.0,29.5,28.3$, 25.5, 23.1, 23.0, 22.4, 20.6, 13.8 .

IR (film, $\mathbf{c m}^{\mathbf{- 1}}$ ) 2931, 2861, 1753, 1365, 1231, 1093. 
HRMS(ESI) Calc. for $\mathrm{C}_{27} \mathrm{H}_{40} \mathrm{O}_{8} \mathrm{Na}(\mathrm{M}+\mathrm{Na}): 515.2615$; found : $515.2611 ;[\boldsymbol{\alpha}]^{\mathbf{2 6}}{ }_{\mathbf{D}}=-$ $28.0^{\circ}\left(c 0.1, \mathrm{CH}_{2} \mathrm{Cl}_{2}\right)$.

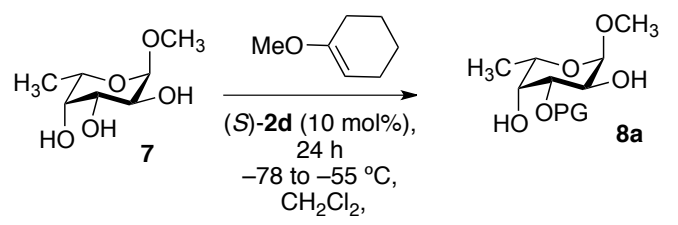

Using the above fucose Triol $(20 \mathrm{mg}, 0.1123 \mathrm{mmol}, 1$ equiv.) as the starting material, synthesis of the acetal was accomplished by following general procedure A. This product was purified by flash column chromatography $(2 / 3$ Hexanes/Ethyl acetate $+1 \%$ triethylamine) to afford the acetal as white solid ( $16.8 \mathrm{mg}, 51.5 \%, \mathrm{C} 3: \mathrm{C} 3: \mathrm{C} 4=\mathrm{C} 3$ only).

${ }^{1}$ H NMR (400 MHz, CD ODD $_{3} \delta 4.62(\mathrm{~d}, J=3.8 \mathrm{~Hz}, 1 \mathrm{H}), 3.97(\mathrm{dd}, J=10.2,3.2 \mathrm{~Hz}, 1 \mathrm{H})$, 3.88 (q, $J=6.6 \mathrm{~Hz}, 1 \mathrm{H}), 3.76$ (dd, $J=10.1,3.8 \mathrm{~Hz}, 1 \mathrm{H}), 3.68(\mathrm{~d}, J=2.8 \mathrm{~Hz}, 1 \mathrm{H}), 3.36$ (s, $3 \mathrm{H}), 3.26(\mathrm{~s}, 3 \mathrm{H}), 1.89$ (d, $J=12.7 \mathrm{~Hz}, 2 \mathrm{H}), 1.70-1.47$ (m, 6H), $1.46-1.34(\mathrm{~m}, 2 \mathrm{H})$, $1.31-1.22(\mathrm{~m}, 2 \mathrm{H}), 1.18(\mathrm{~d}, J=6.6 \mathrm{~Hz}, 3 \mathrm{H})$.

${ }^{13}$ C NMR (100 MHz, CD $_{3}$ OD) $\delta 101.6,100.4,72.2,69.6,67.0,65.7,54.1,48.2,48.0$, 47.8, 47.6, 47.3, 47.1, 46.9, 33.7, 33.5, 25.1, 22.7, 22.6, 15.3.

IR (film, $\mathbf{~ c m}^{-1}$ ) 3473, 2932, 1362, 1161, 1095, 1045, 950, 928. HRMS(ESI) Calc. for $\mathrm{C}_{14} \mathrm{H}_{26} \mathrm{O}_{6} \mathrm{Na}(\mathrm{M}+\mathrm{Na}): 313.1622$; found : $313.1621 ;[\boldsymbol{\alpha}]^{\mathbf{2 6}}{ }_{\mathbf{D}}=-109.8^{\circ}\left(c 0.3, \mathrm{CH}_{2} \mathrm{Cl}_{2}\right)$.

Determination of $\mathrm{C} 2 / \mathrm{C} 3 / \mathrm{C} 4$ ratios was done using COSY experiments of acetylated product.

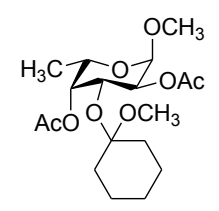

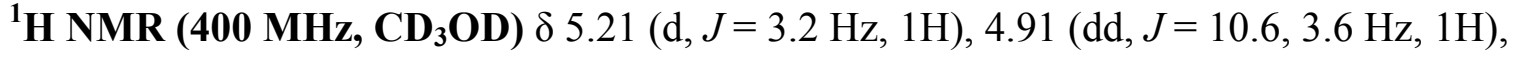
$4.81(\mathrm{~d}, J=3.5 \mathrm{~Hz}, 1 \mathrm{H}), 4.38(\mathrm{dd}, J=10.6,3.3 \mathrm{~Hz}, 1 \mathrm{H}), 4.06$ (q, $J=6.5 \mathrm{~Hz}, 1 \mathrm{H}), 3.34$ (s, $3 \mathrm{H}), 3.20(\mathrm{~s}, 3 \mathrm{H}), 2.12(\mathrm{~s}, 3 \mathrm{H}), 2.04(\mathrm{~s}, 3 \mathrm{H}), 1.90-1.76(\mathrm{~m}, 2 \mathrm{H}), 1.63-1.50(\mathrm{~m}, 4 \mathrm{H})$, $1.43-1.26(\mathrm{~m}, 4 \mathrm{H}), 1.06(\mathrm{~d}, J=6.5 \mathrm{~Hz}, 3 \mathrm{H})$.

${ }^{13}$ C NMR (100 MHz, $\mathbf{C D}_{3}$ OD) $\delta$ 171.1, 170.7, 101.8, 97.4, 74.5, 69.9, 64.7, 64.6, 54.2, 48.2, 48.0, 47.8, 47.6, 47.3, 47.1, 46.9, 33.8, 33.2, 24.9, 22.7, 22.7, 19.6, 19.3, 15.1 . 
IR (film, $\mathbf{c m}^{-1}$ ) 2932，1741，1369，1233，1053，928. HRMS(ESI) Calc. for $\mathrm{C}_{18} \mathrm{H}_{30} \mathrm{O}_{8} \mathrm{Na}(\mathrm{M}+\mathrm{Na}): 397.1833$; found : 397.1832; $[\boldsymbol{\alpha}]^{\mathbf{2 6}}{ }_{\mathbf{D}}=-34.1^{\circ}\left(c 0.3, \mathrm{CH}_{2} \mathrm{Cl}_{2}\right)$.

\section{Acetalization Reactions: Time Dependent study}

An oven dried and nitrogen flushed $25 \mathrm{~mL}$ round bottom flask was charged with thioglycoside diol $1 \mathbf{c}^{5}$ (60 $\mathrm{mg}, 0.17 \mathrm{mmol}, 1$ equiv.), (which was previously dried by azeotropic removal of moisture with toluene), anhydrous dichloromethane $(4 \mathrm{~mL})$ and activated $4 \AA$ molecular sieves. This mixture was submerged in dry ice/acetone bath and 1-methoxycylohexene ( $25.2 \mu \mathrm{L}, 0.20 \mathrm{mmol}, 1.2$ equiv.), followed by $(R)-2 d(3.1 \mathrm{mg}$, $0.003 \mathbf{m m o l}, 2 \mathrm{~mol} \%$ ) was added. The formation of $\mathbf{5} \mathbf{c}$ and its respective $\mathrm{C} 2 / \mathrm{C} 3$ ratio was monitored every hour by quenching about $0.2 \mathrm{~mL}$ of the reaction mixture with triethylamine, then concentrating it in vacuo and analyzing it by ${ }^{1} \mathrm{HNMR}$. This routine was repeated every hour for the next 6 hours. The remaining reaction mixture was transferred into a Neslab CB 80 immersion cooler and stirred at $-50{ }^{\circ} \mathrm{C}$ overnight. The formation of $\mathbf{5 c}$ as well as its $\mathrm{C} 2 / \mathrm{C} 3$ ratio as the reaction progresses is displayed below.

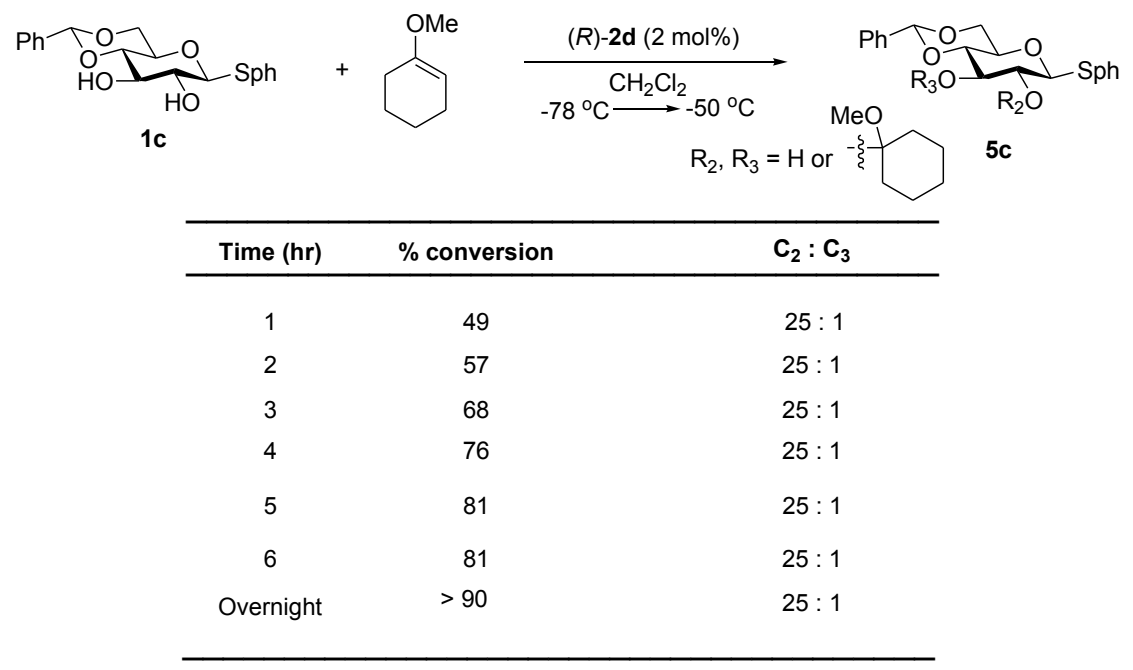

\section{Meso Diol Desymmetrization Studies}




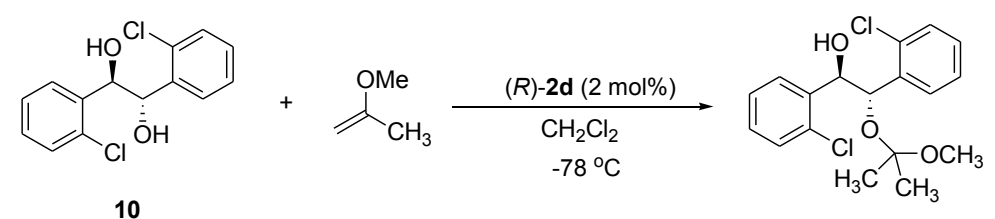

11

An oven dried and nitrogen flushed $10 \mathrm{~mL}$ round bottom flask was charged with meso1,2-bis(2-chlorophenyl)-1,2-ethane diol 10 (15.9 mg, $0.056 \mathrm{mmol}, 1$ equiv.), (which was previously dried by azeotropic removal of moisture with toluene), anhydrous dichloromethane $(1.3 \mathrm{~mL})$ and activated $4 \AA$ molecular sieves. This mixture was submerged in dry ice/acetone bath and 2-methoxypropene $(6.4 \mu \mathrm{L}, 0.067 \mathrm{mmol}, 1.2$ equiv.), followed by $(R)-\mathbf{2 d}(1.1 \mathrm{mg}, 0.001 \mathrm{mmol}, 2 \mathrm{~mol} \%)$ was added. The resulting reaction mixture was transferred into a Neslab CB 80 immersion cooler and stirred at -78 ${ }^{\circ} \mathrm{C}$. When the reaction was completed as monitored by TLC, the reaction mixture was quenched with triethylamine, and concentrated in vacuo to form pale yellow oil as the crude product. The crude product 11 was subsequently analysed by HPLC (99/1 Hexanes/Isopropanol with CHIRALPAK AD-H Column, $\mathrm{R}_{\mathrm{fl}}=18.4$ min, $\mathrm{R}_{\mathrm{f} 2}=21.9$ ) to determine its enantiomeric ratio. The crude product was then purified by silica gel flash column chromatography (5/1 Hexanes/Ethyl acetate $+1 \%$ triethylamine) to afford pure 11 as a pale yellow oil $(16.8 \mathrm{mg}, 85 \%$, e.r. $=86: 14)$.

${ }^{1} \mathbf{H}$ NMR (500 MHz, $\left.\mathbf{C}_{\mathbf{6}} \mathbf{D}_{\mathbf{6}}\right) \delta 7.57(\mathrm{~d}, J=7.7 \mathrm{~Hz}, 1 \mathrm{H}), 7.17-7.13(\mathrm{~m}, 1 \mathrm{H}), 7.08(\mathrm{dd}, J=$ 9.6, 3.6 Hz, 1H), $6.96-6.85(\mathrm{~m}, 2 \mathrm{H}), 6.76-6.62(\mathrm{~m}, 3 \mathrm{H}), 5.86(\mathrm{~d}, J=3.6 \mathrm{~Hz}, 1 \mathrm{H}), 5.58$ (d, $J=3.7 \mathrm{~Hz}, 1 \mathrm{H}), 2.96$ (s, 3H), 2.40 (s, 1H), 1.23 (s, 3H), 1.02 (s, 3H).

${ }^{13} \mathbf{C}$ NMR (125 MHz, $\left.\mathbf{C}_{6} \mathbf{D}_{6}\right) \delta 137.5,137.3,133.6,133.1,130.5,129.0,128.4,128.3$, $128.1,127.9,127.8,127.7,127.6,127.6,127.5,126.0,125.7,101.4,73.0,70.1,48.8$, 25.4, 24.5.

IR (film, $\mathbf{c m}^{-1}$ ) 3464, 2989, 2940, 1597, 1573, 1471, 1441, 1382, 1209, 1147, 1075, $1053,1029,880$.

$[\boldsymbol{\alpha}]^{\mathbf{2 6}}{ }_{\mathbf{D}}=-7.84^{\circ}\left(c 0.5, \mathrm{CH}_{2} \mathrm{Cl}_{2}\right)$.

\section{Proposed Mechanism}


The tentative mechanism for the regioselective chiral phosphoric acid-catalyzed acetalizations is provided in Scheme 1SI. While the precise nature of the reaction intermediates has yet to be investigated, our preliminary results suggest that the acetalization reactions take place under kinetic control. Although the THP ethers are not prone to acid-promoted migration, the products 5 and $\mathbf{6}$ (Table 3, Manuscript) could in theory equilibrate or further react under the reaction conditions. However, no isomerization of the products (or formation of side-products from $\mathbf{5}$ and $\mathbf{6}$ ) was observed under the reaction conditions. To rationalize the observations, both a concerted mechanism proceeding through $\mathbf{B}$ or a more traditional stepwise mechanism proceeding through A and bmight be proposed (Scheme 1SI). Thus, either phosphoric acid (B) or phosphate anion (C) are involved in the formation of a hydrogen bond with the diol, and the observed regioselectivities likely result from a more favored coordination of the catalyst to the substrate.

\section{Scheme 1SI}

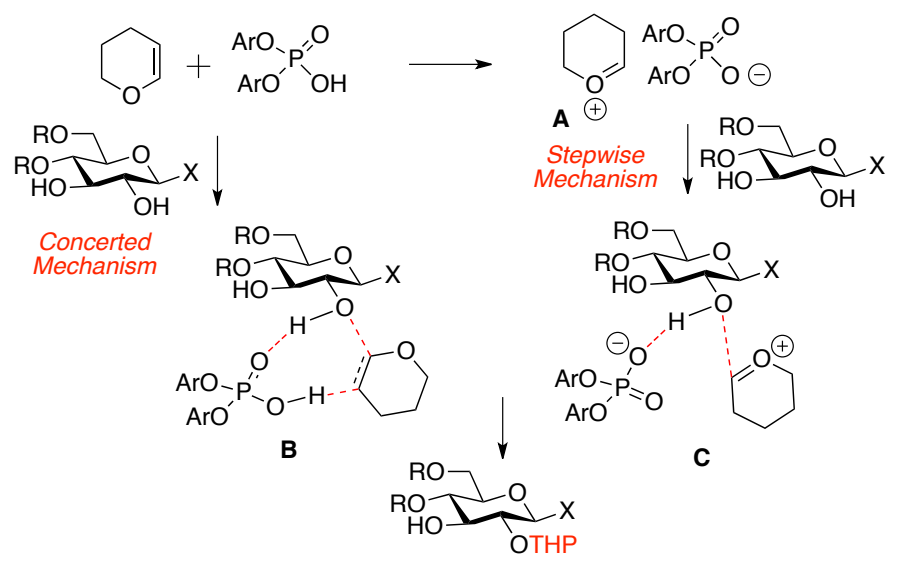




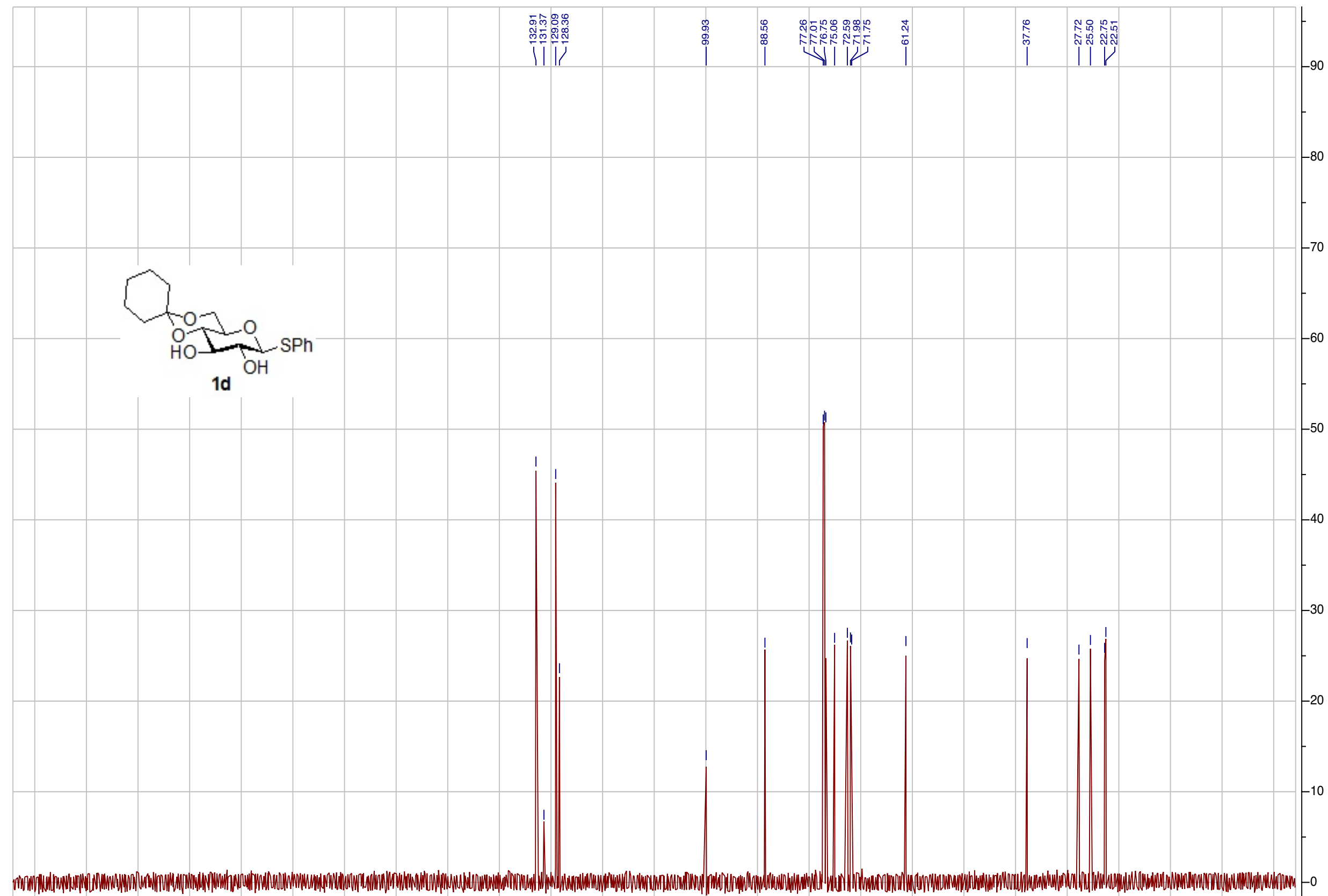

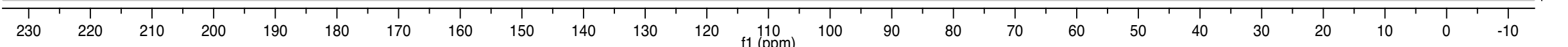




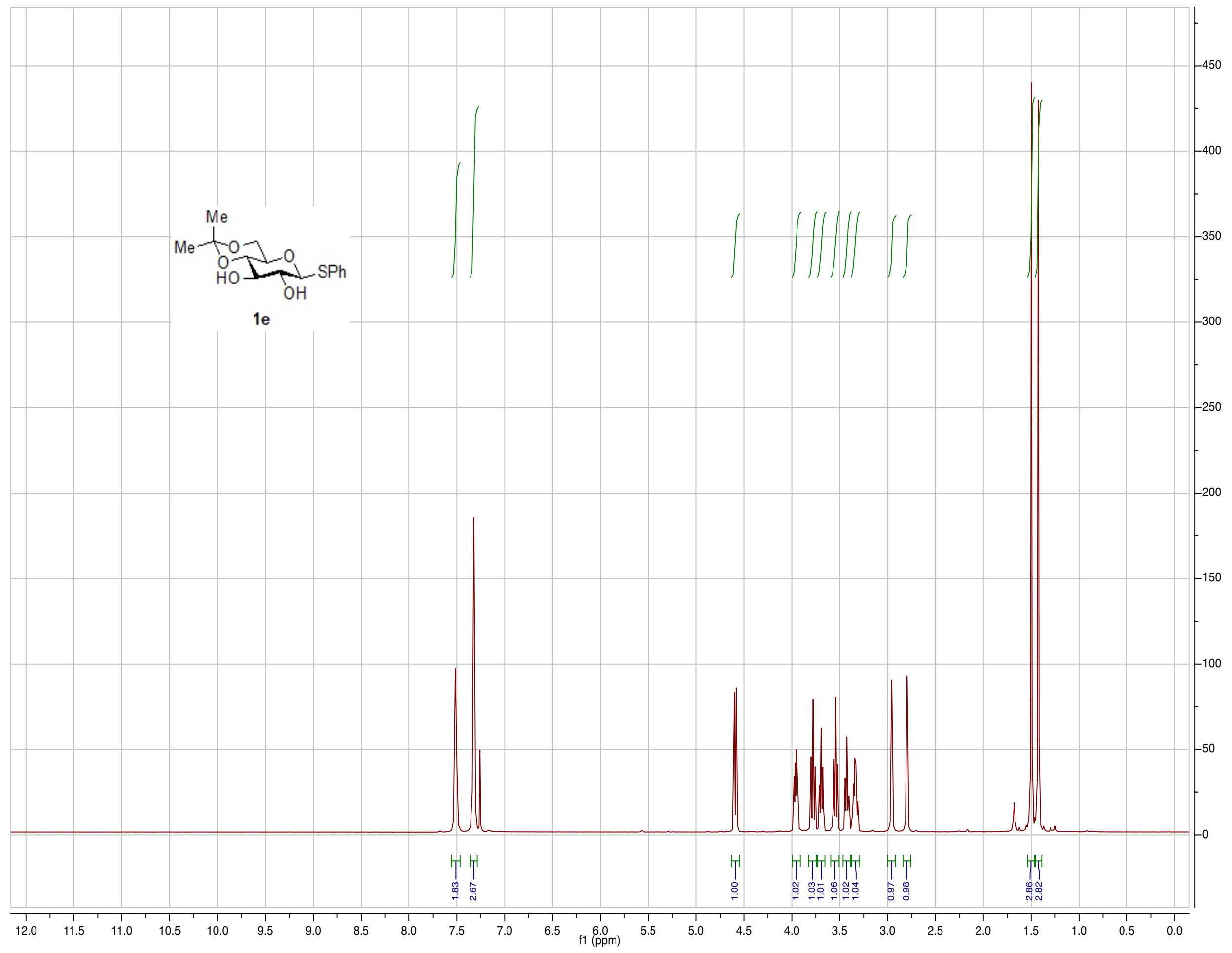




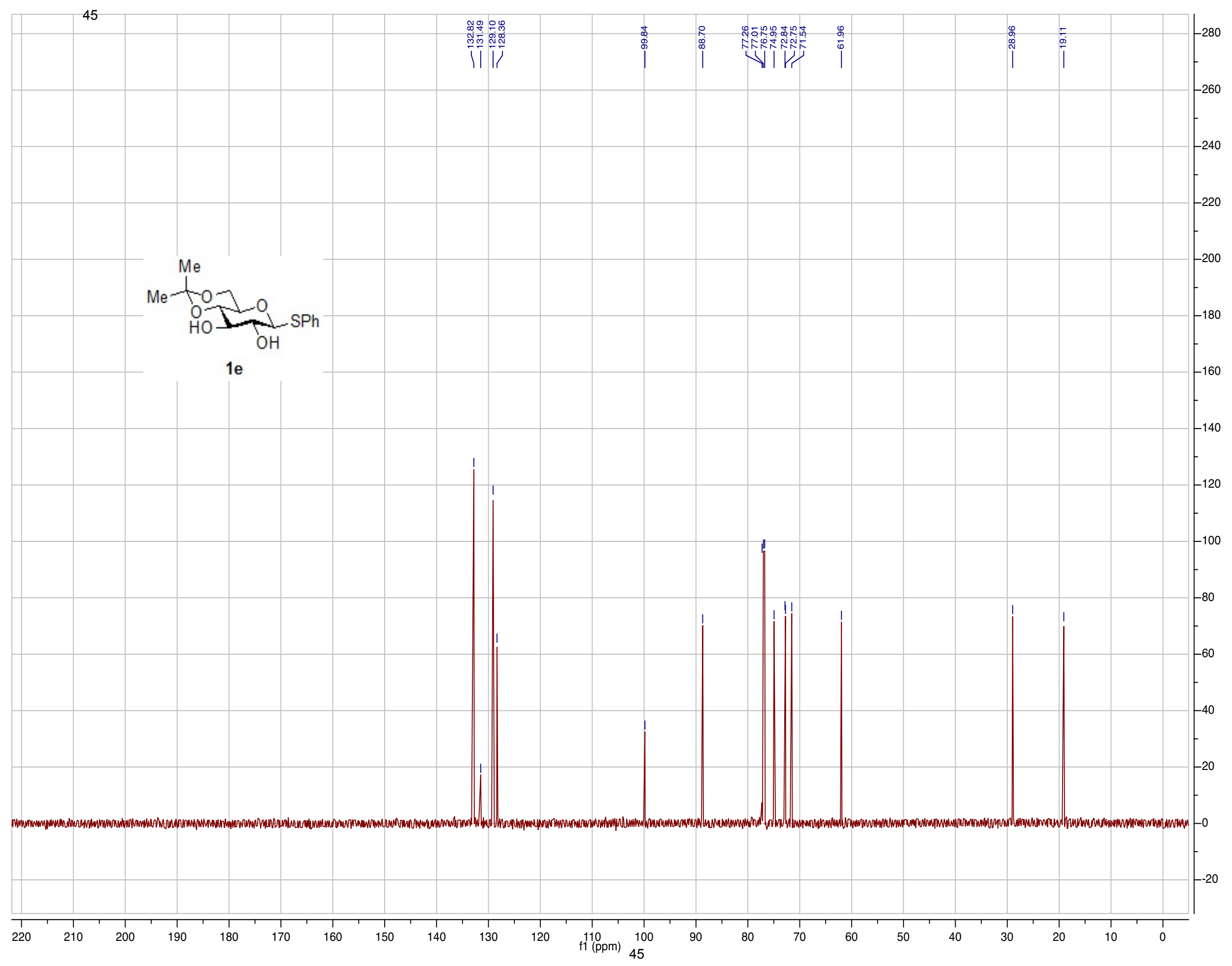




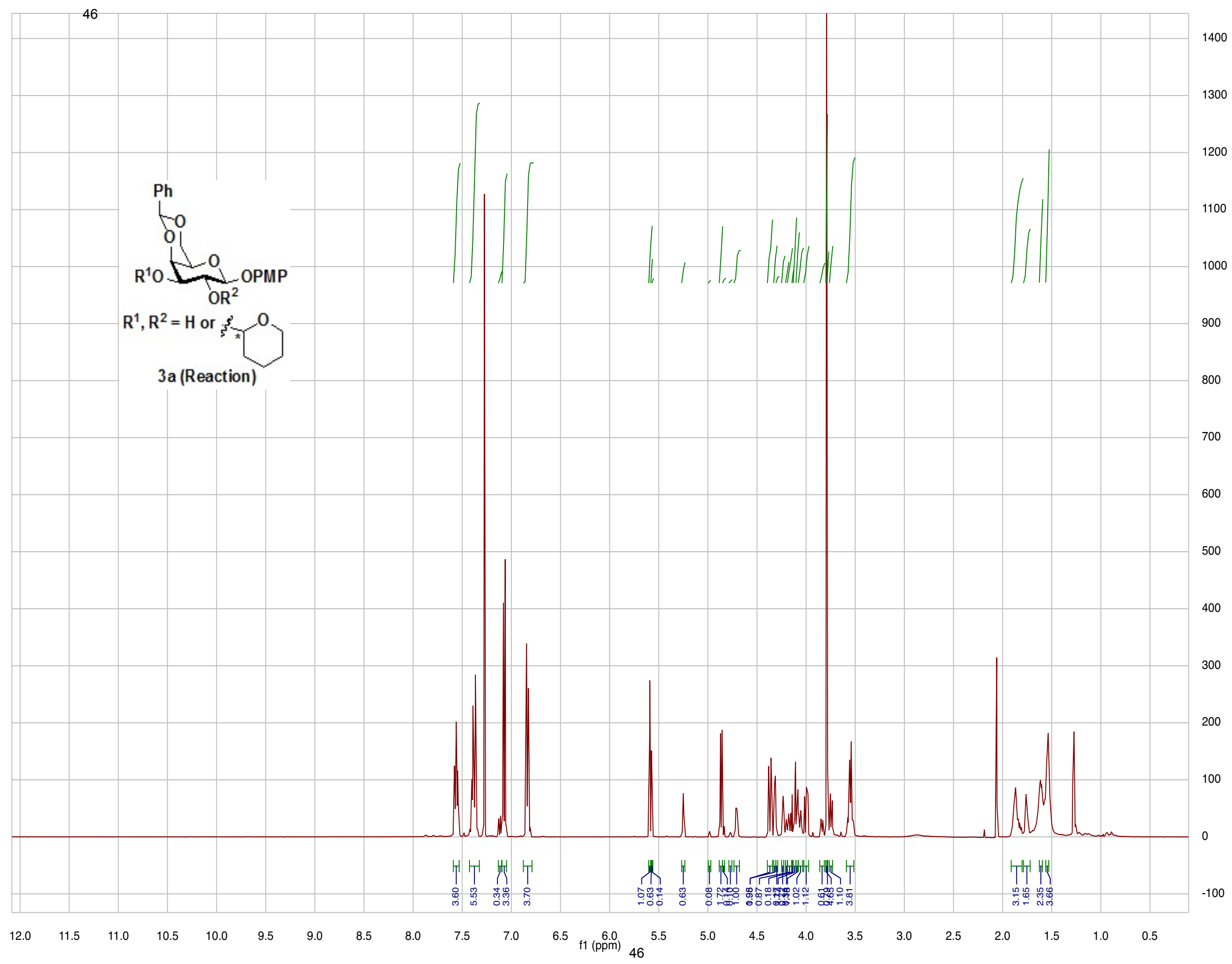




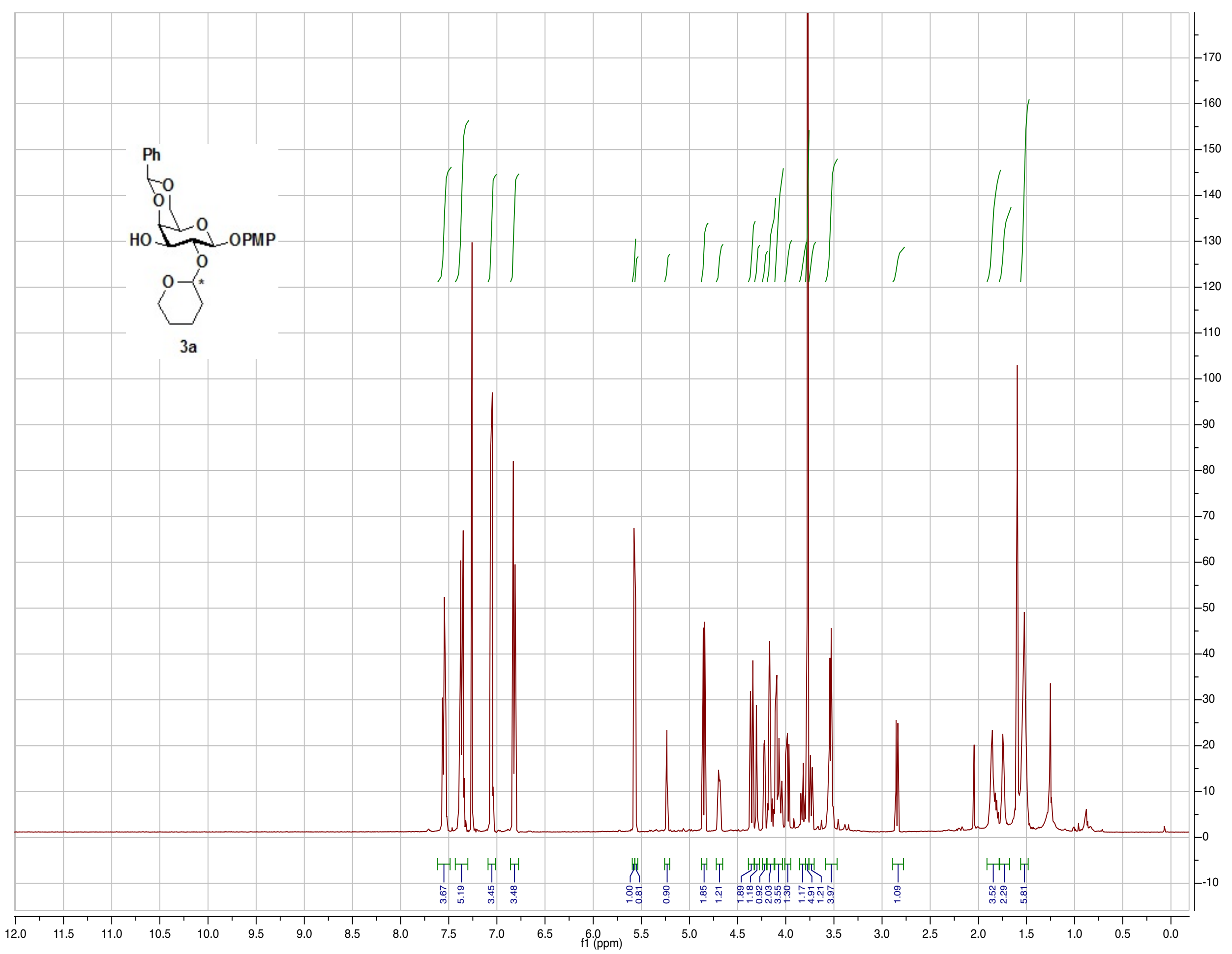




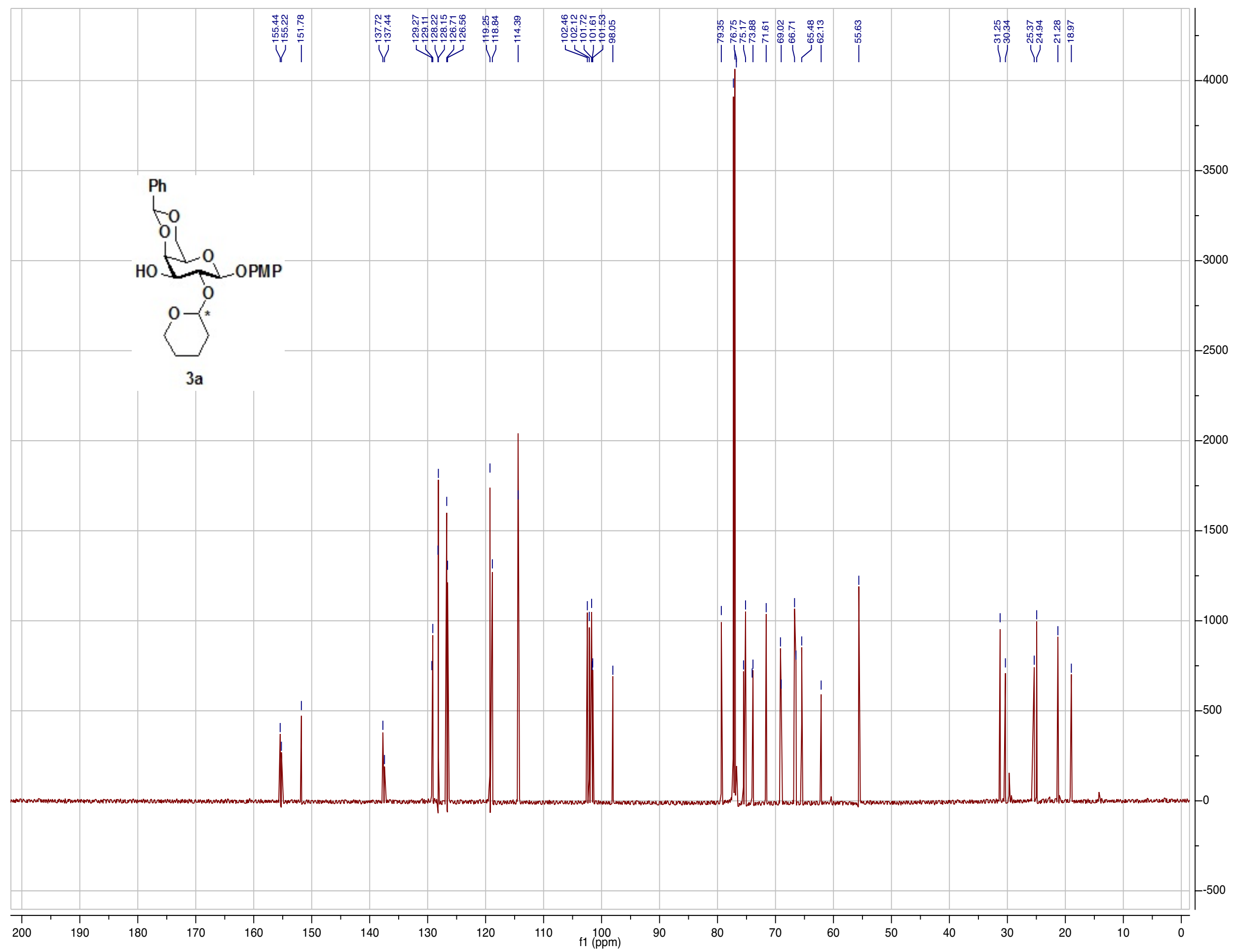




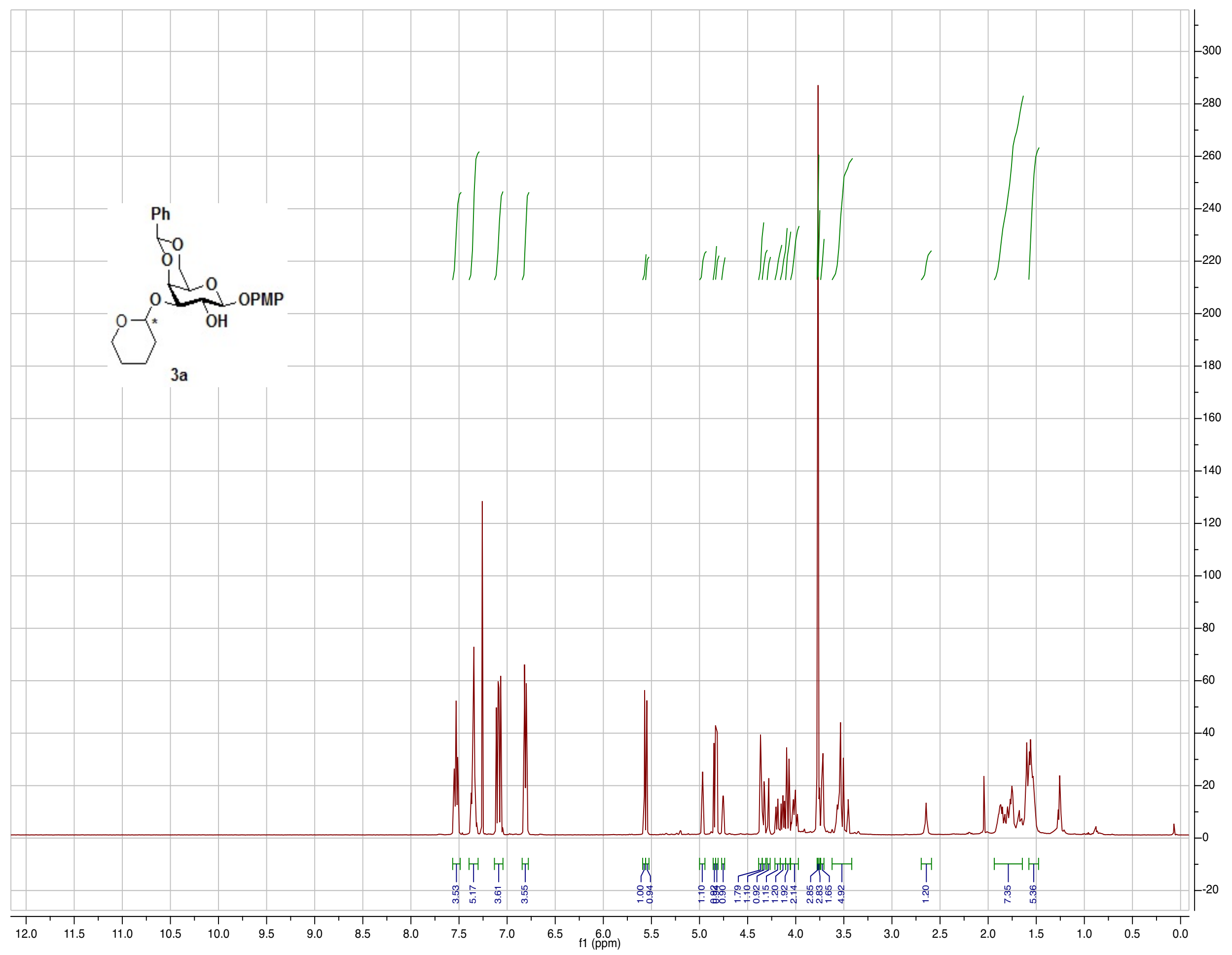




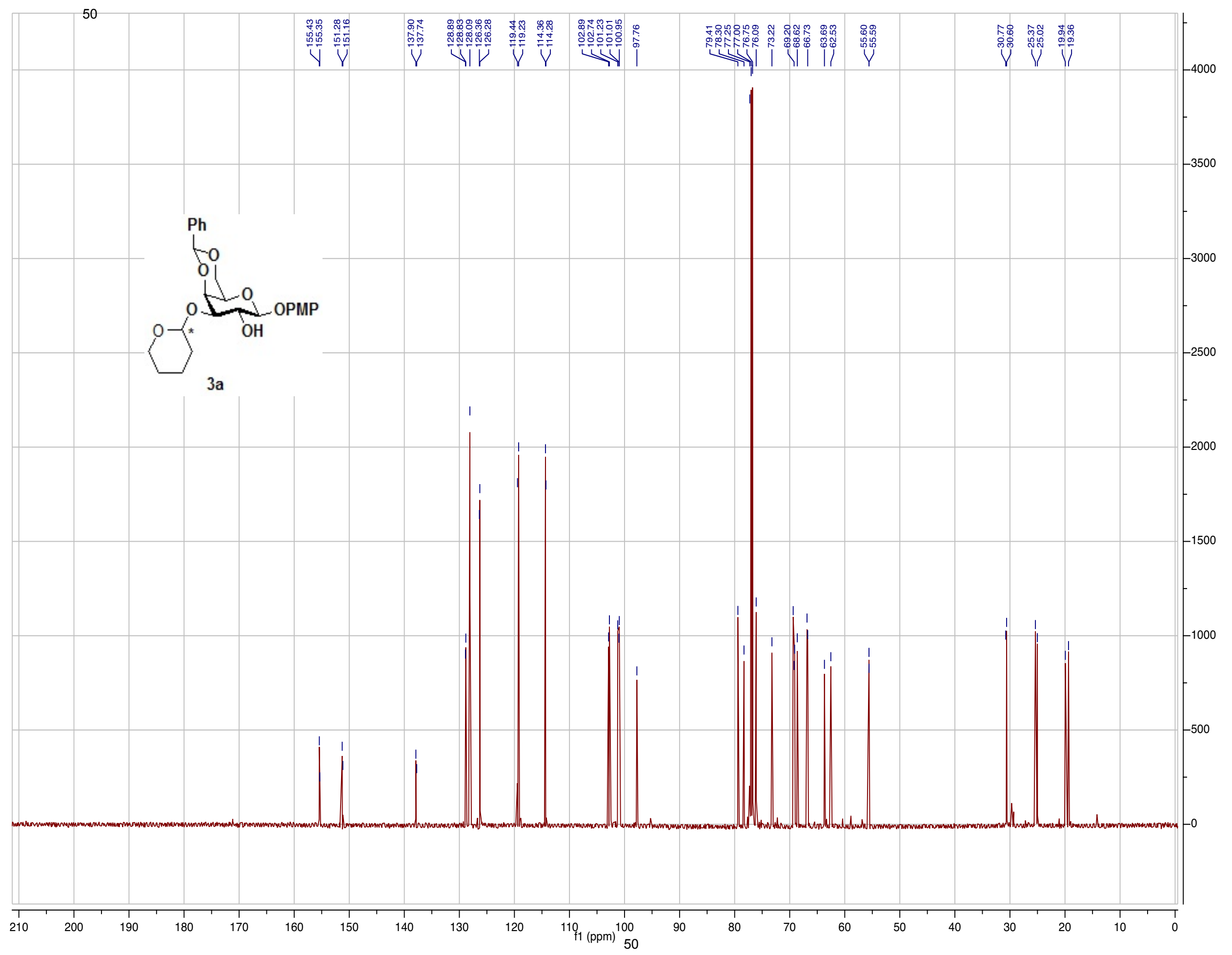




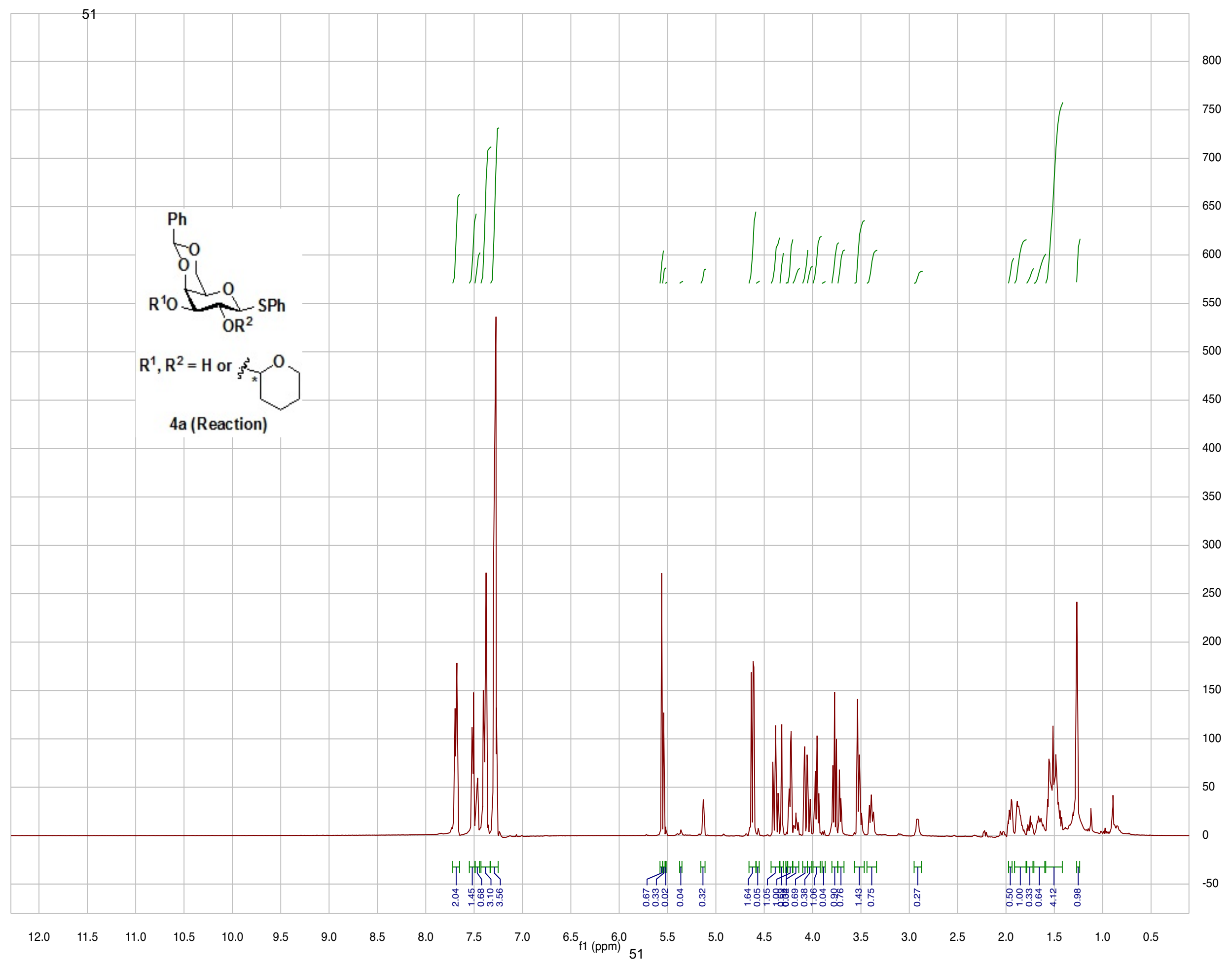




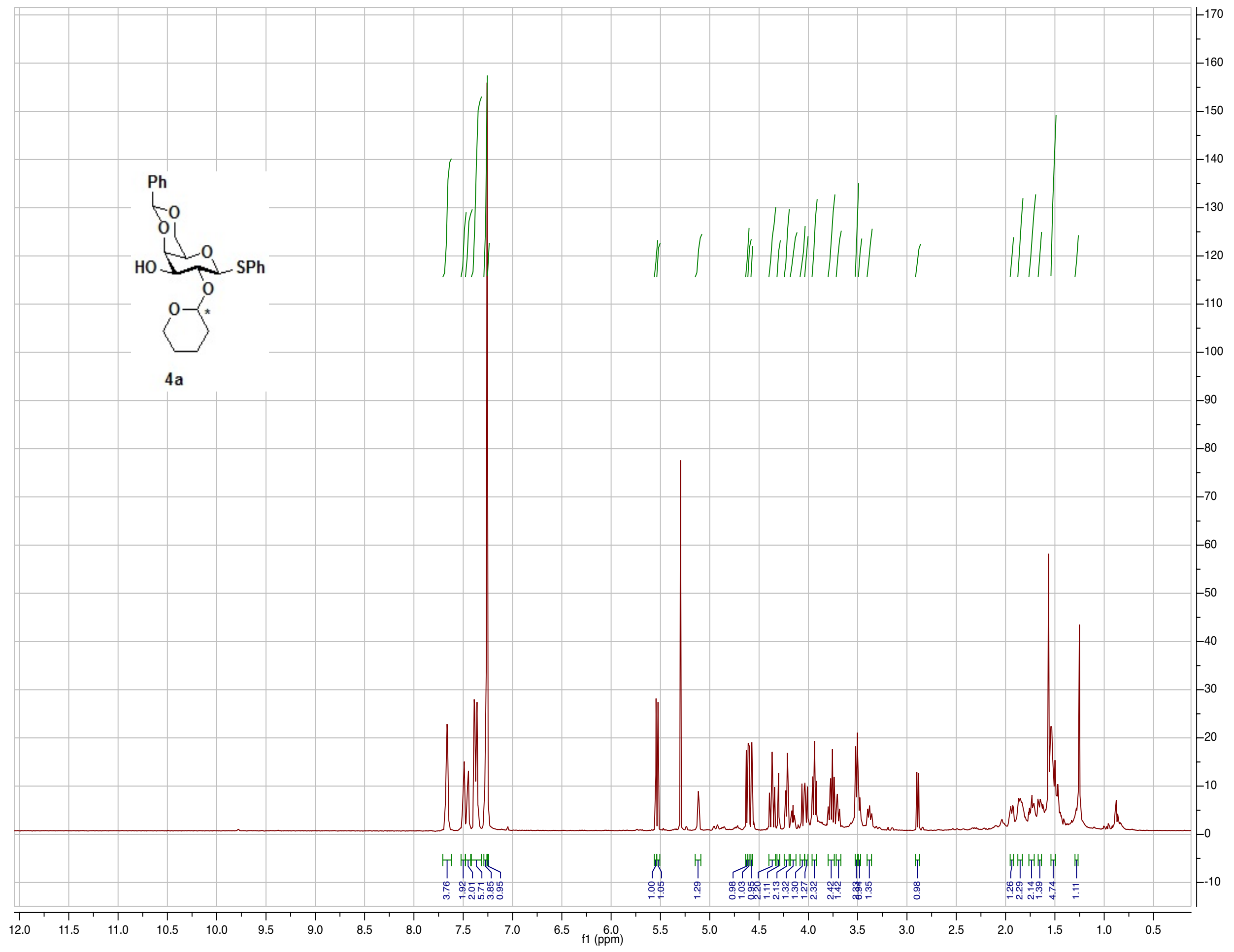




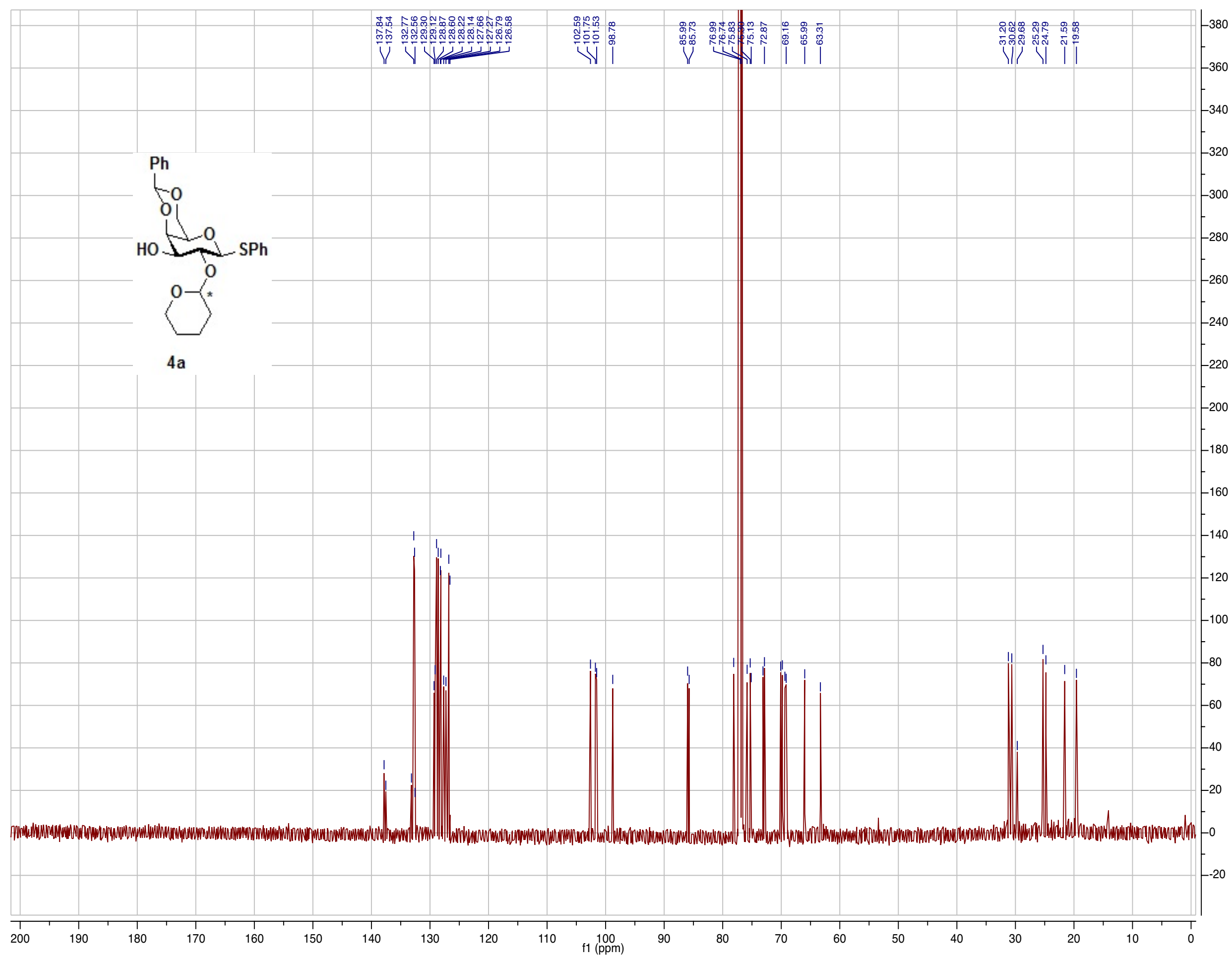




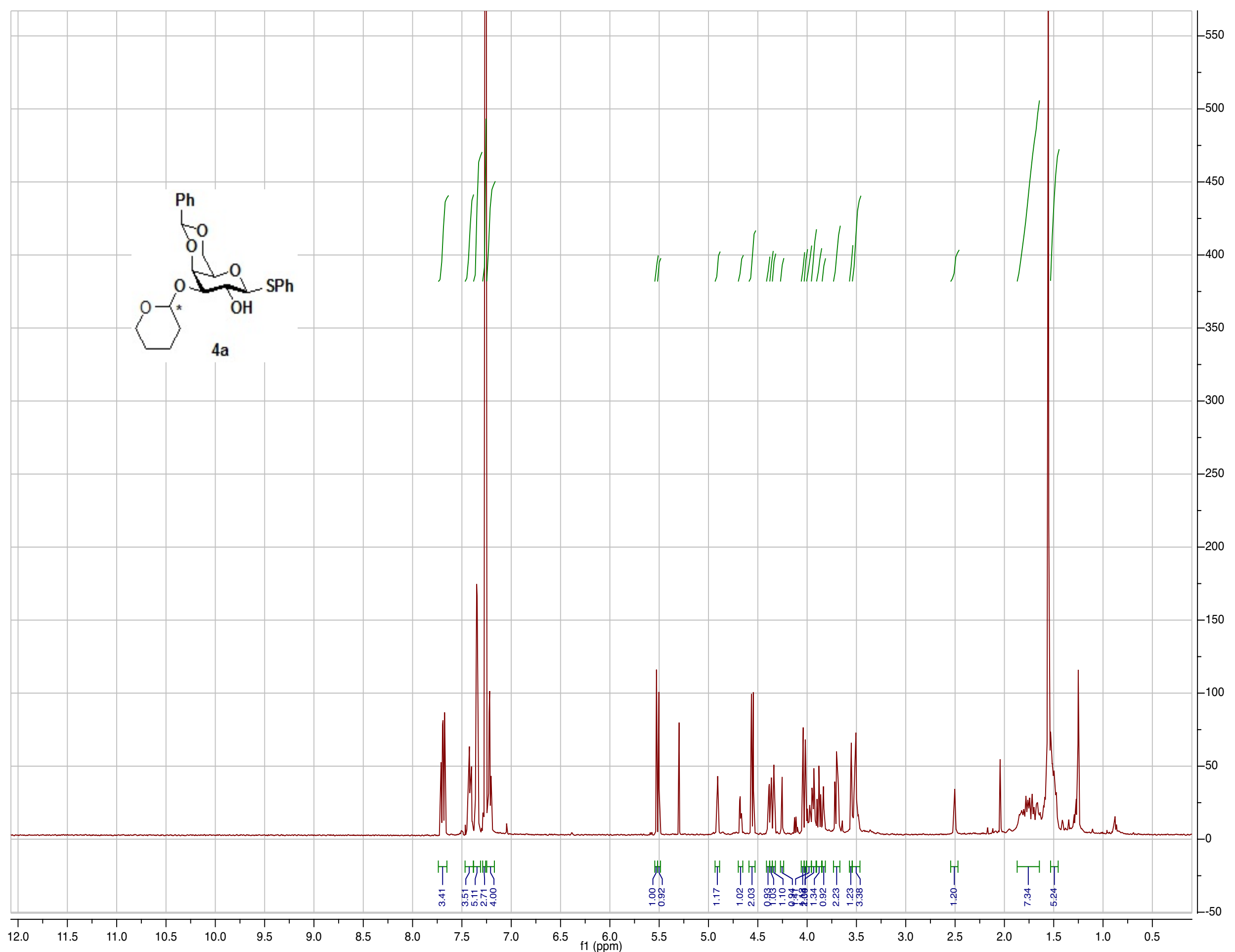




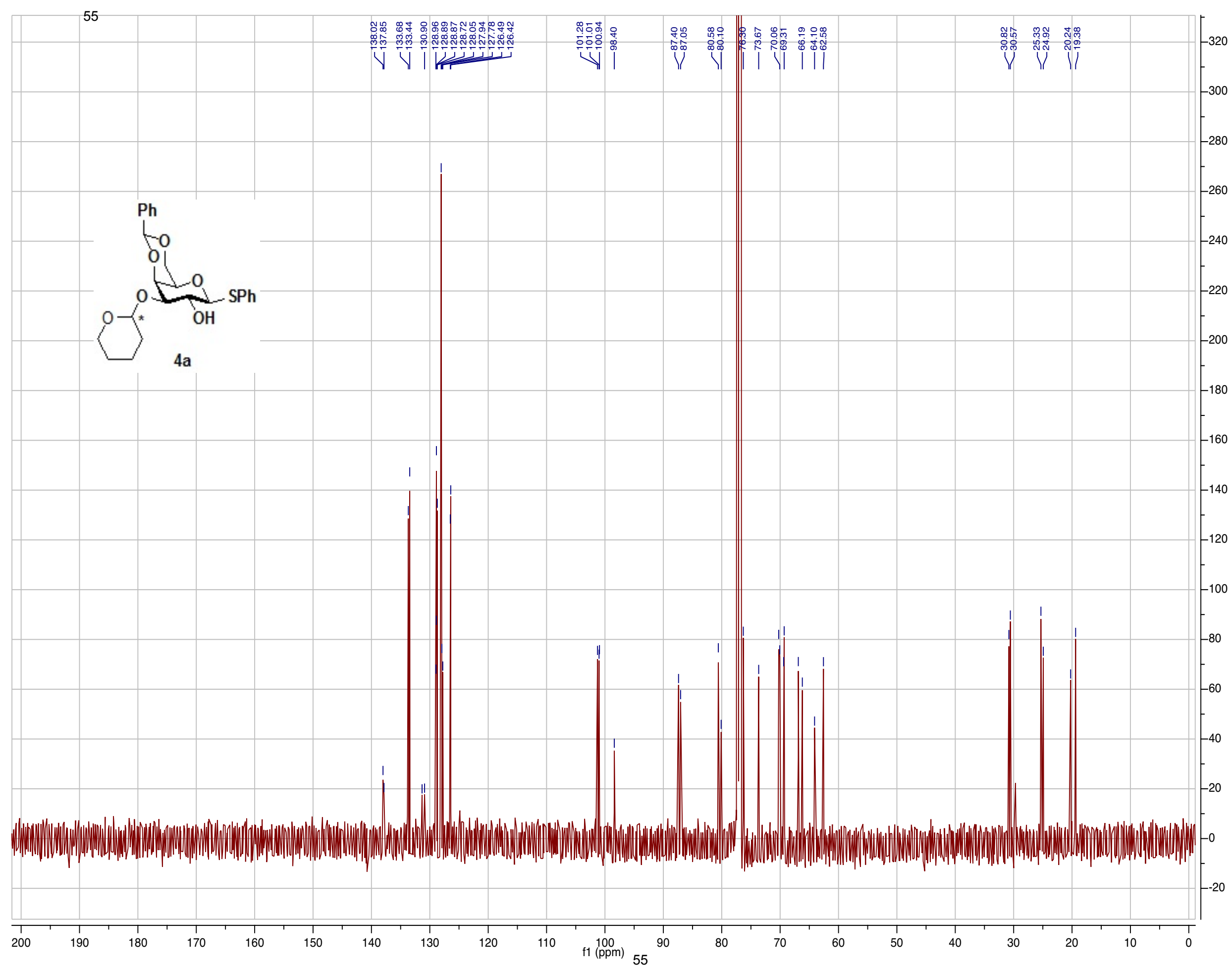




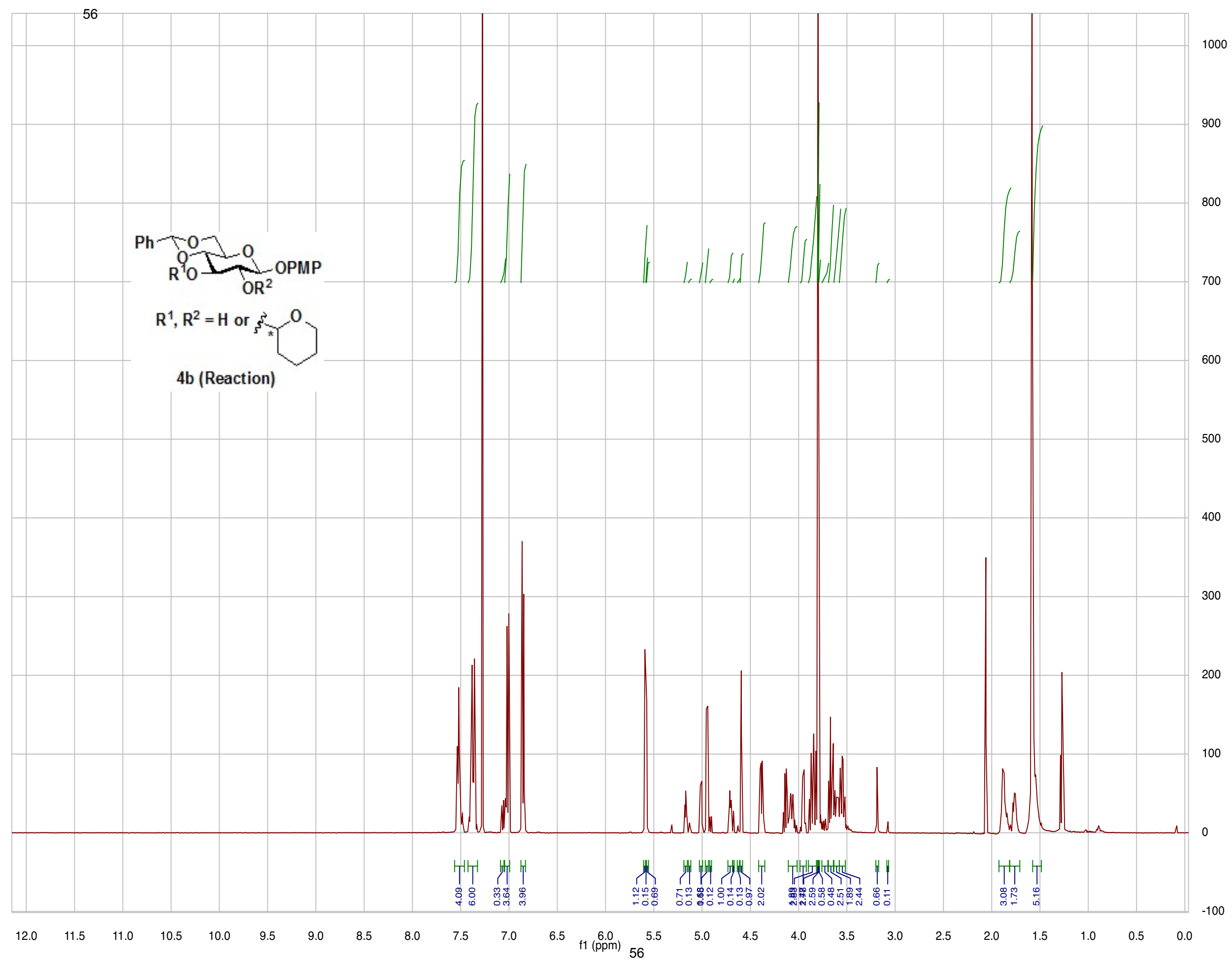




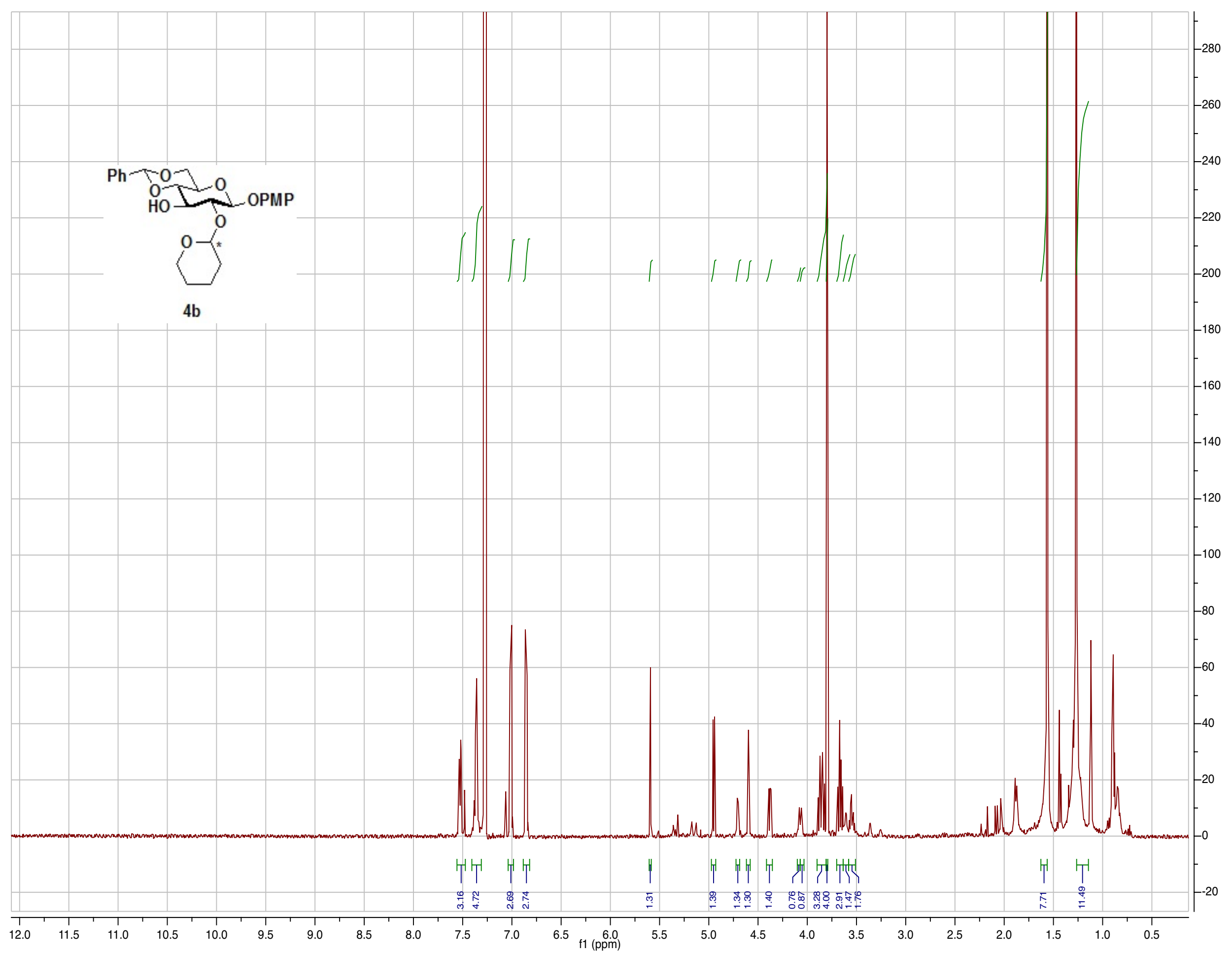




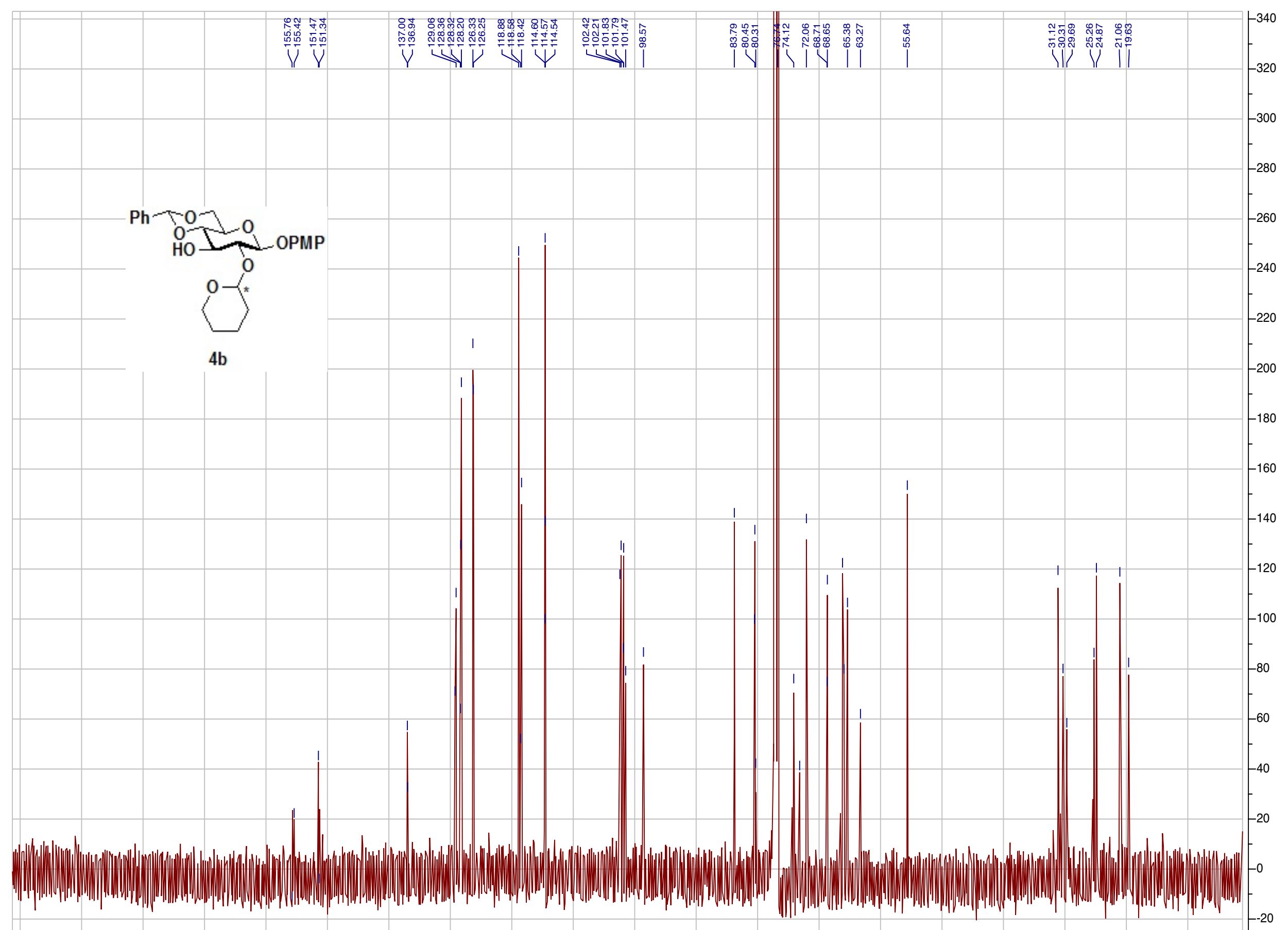




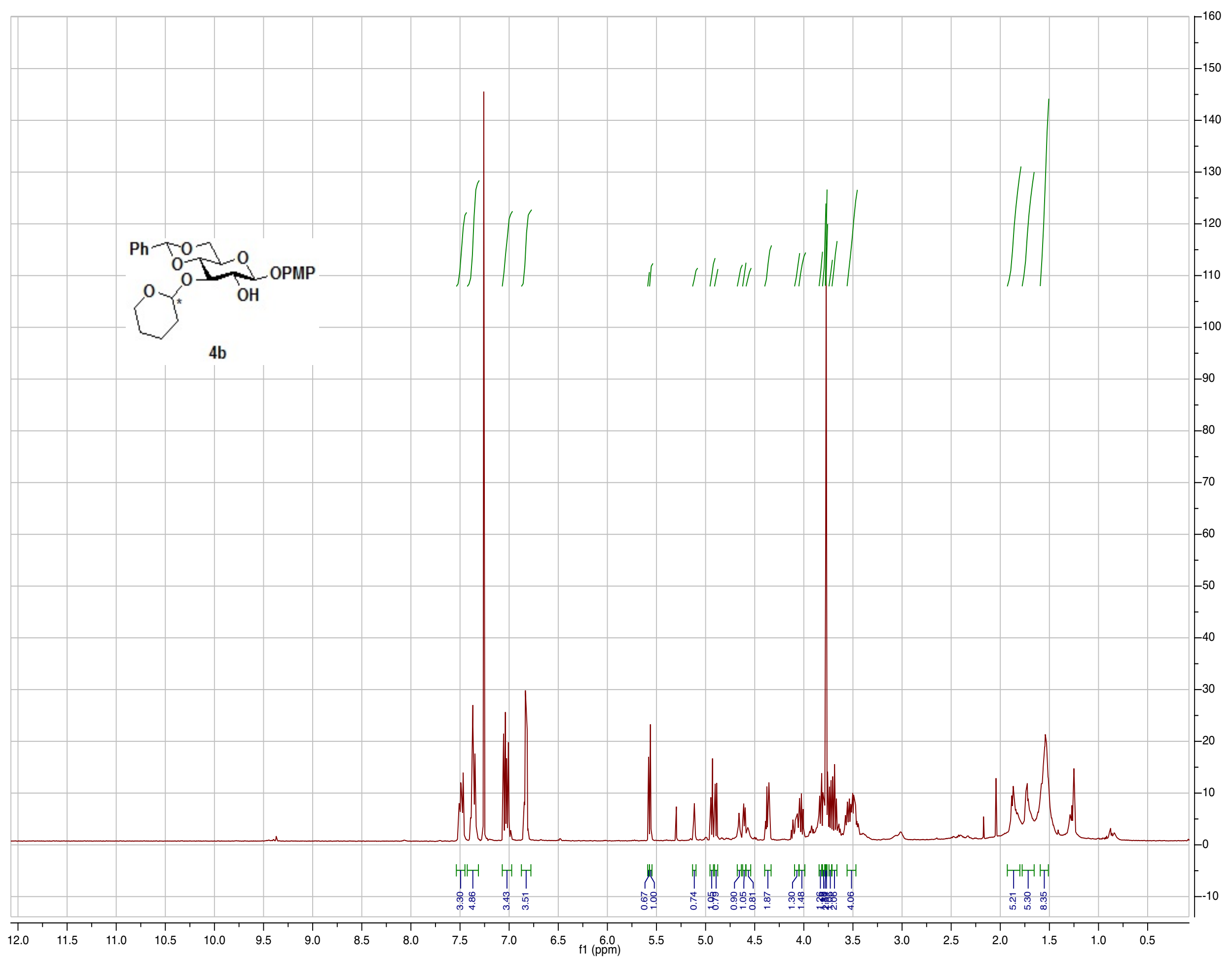



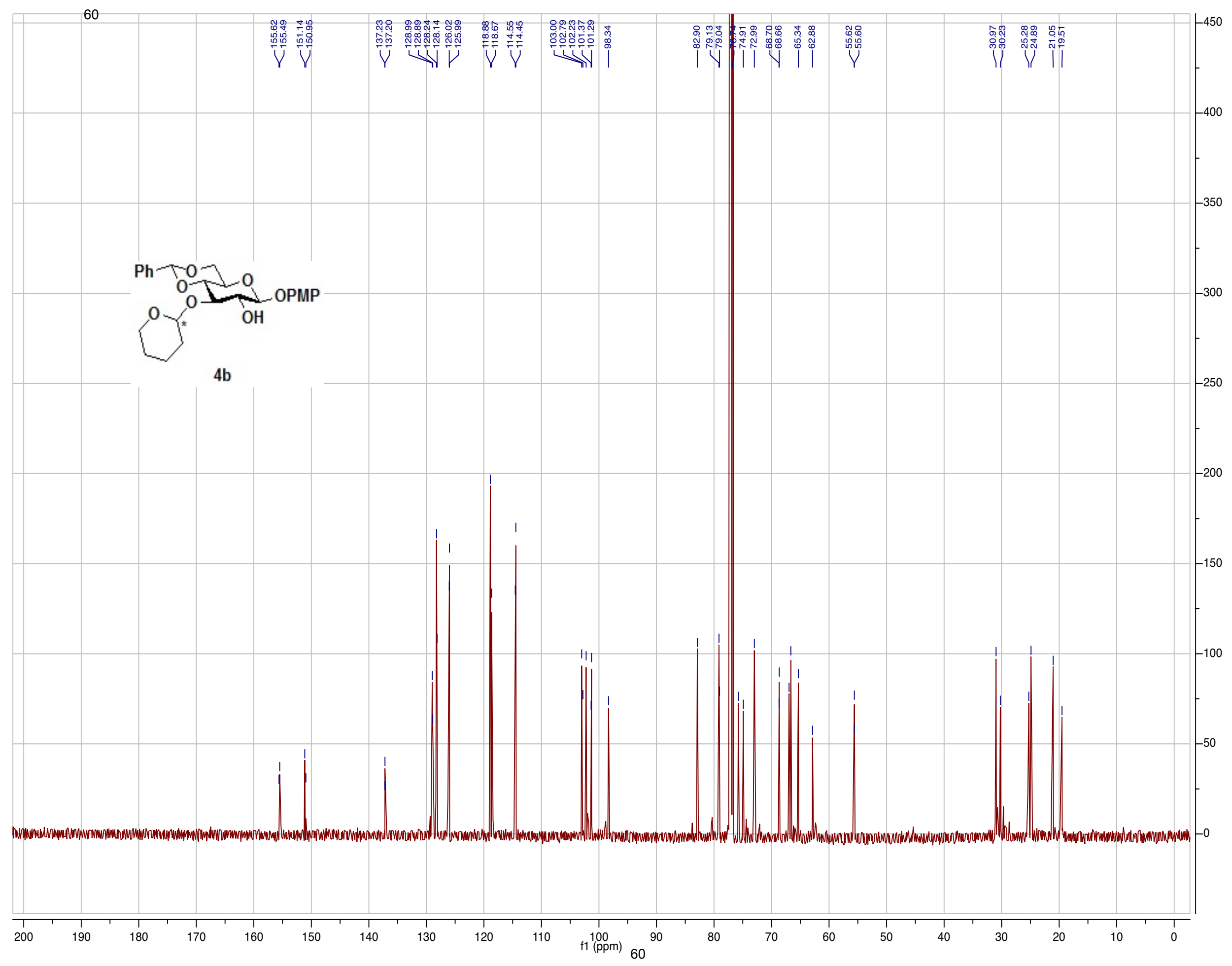


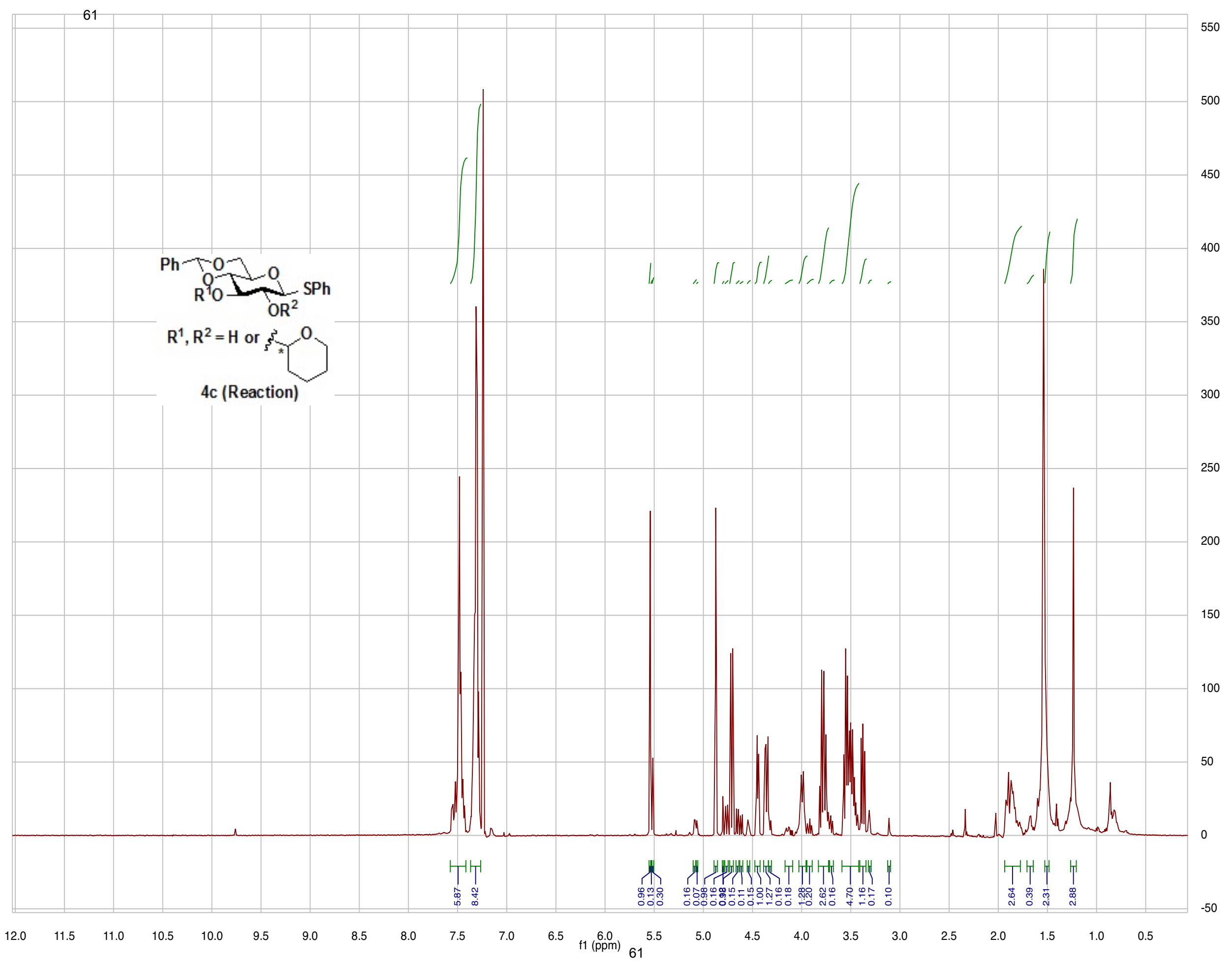




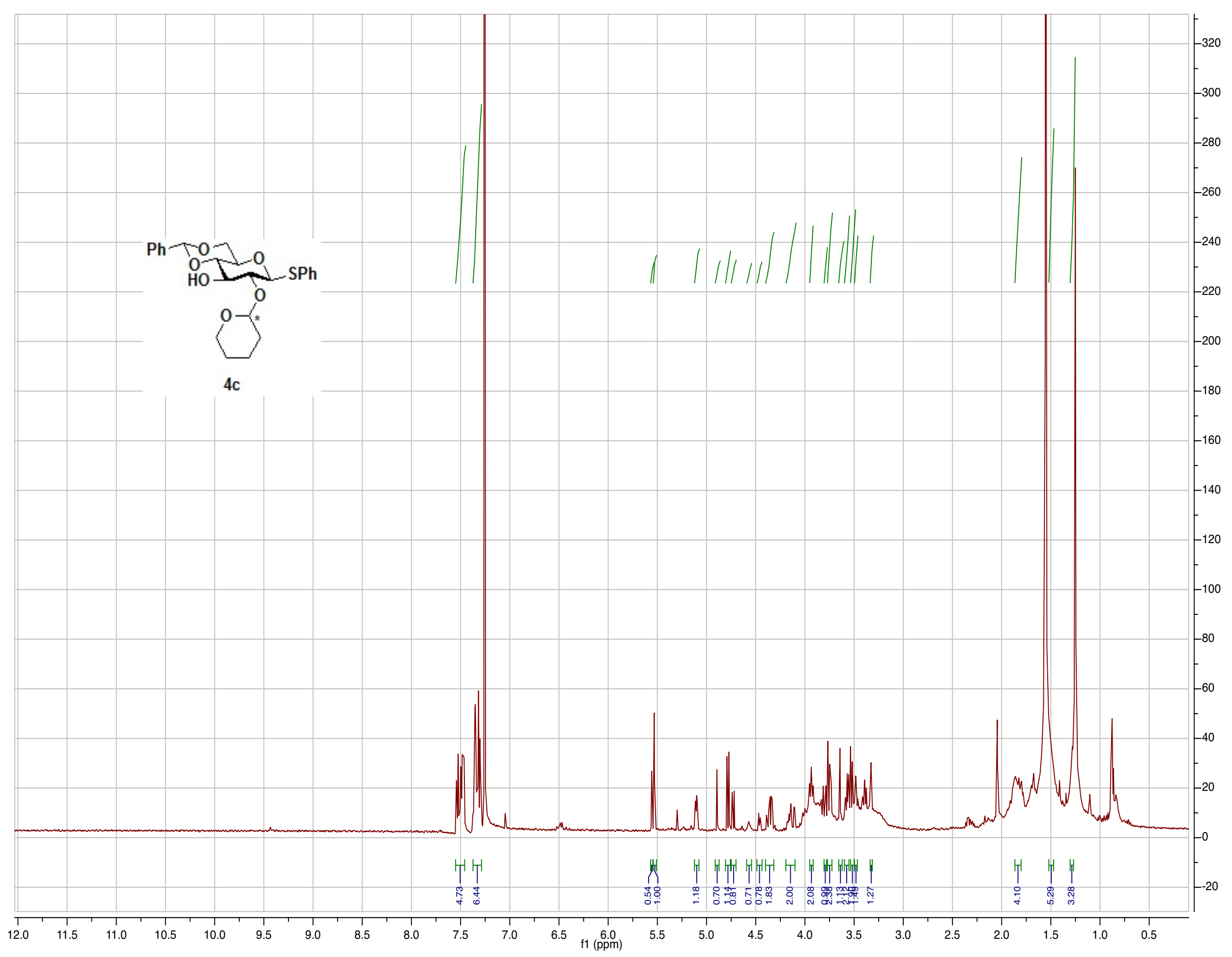




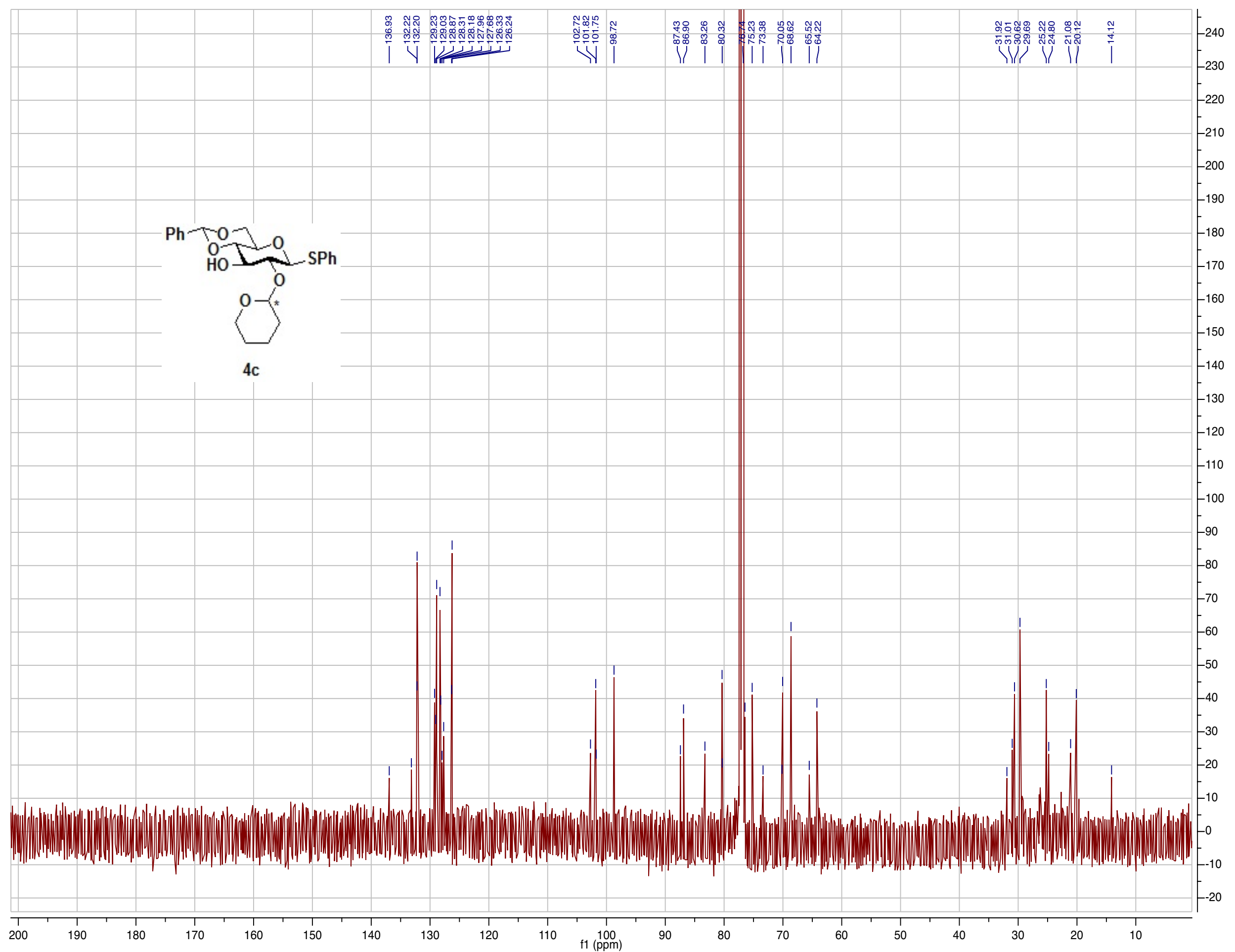




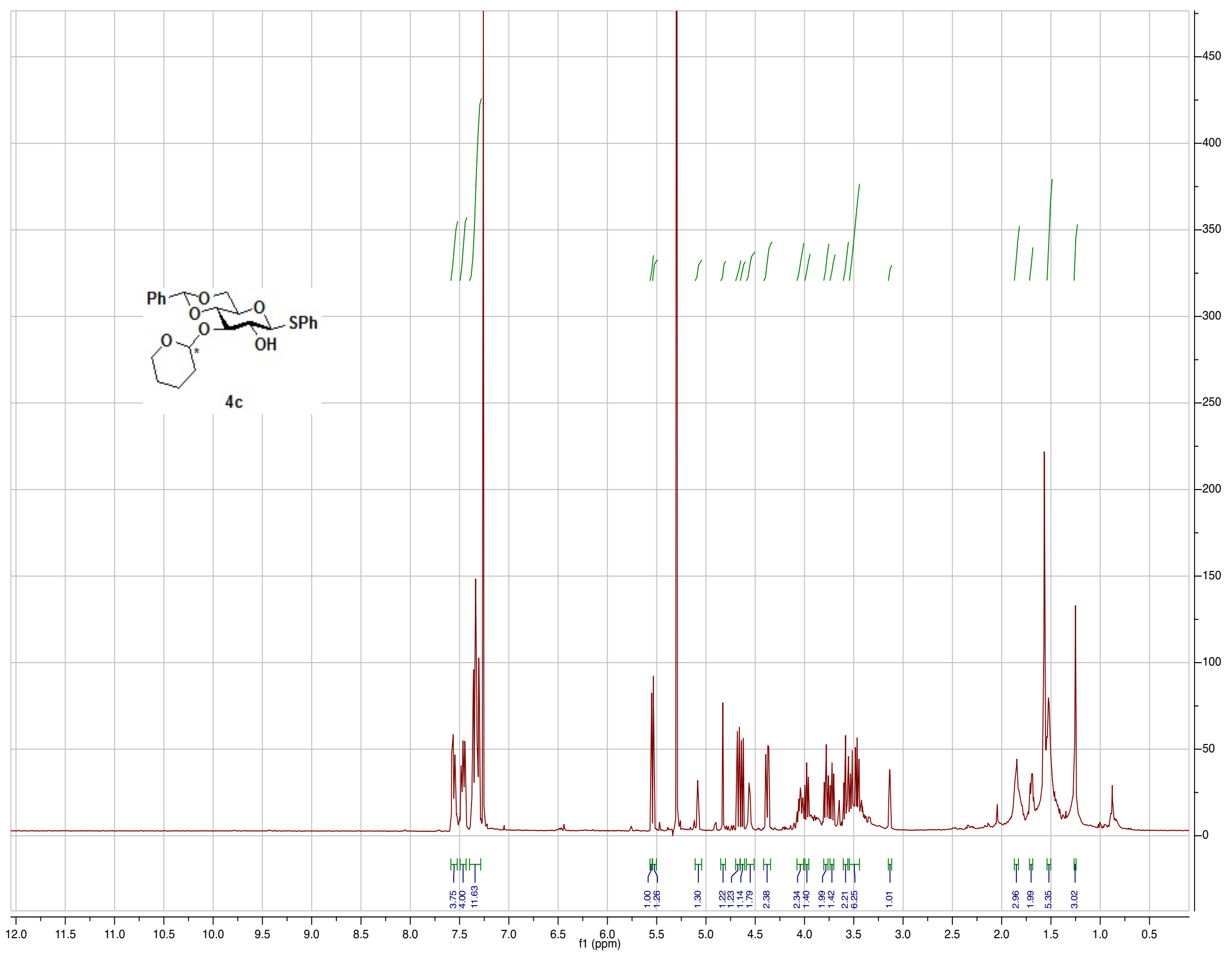




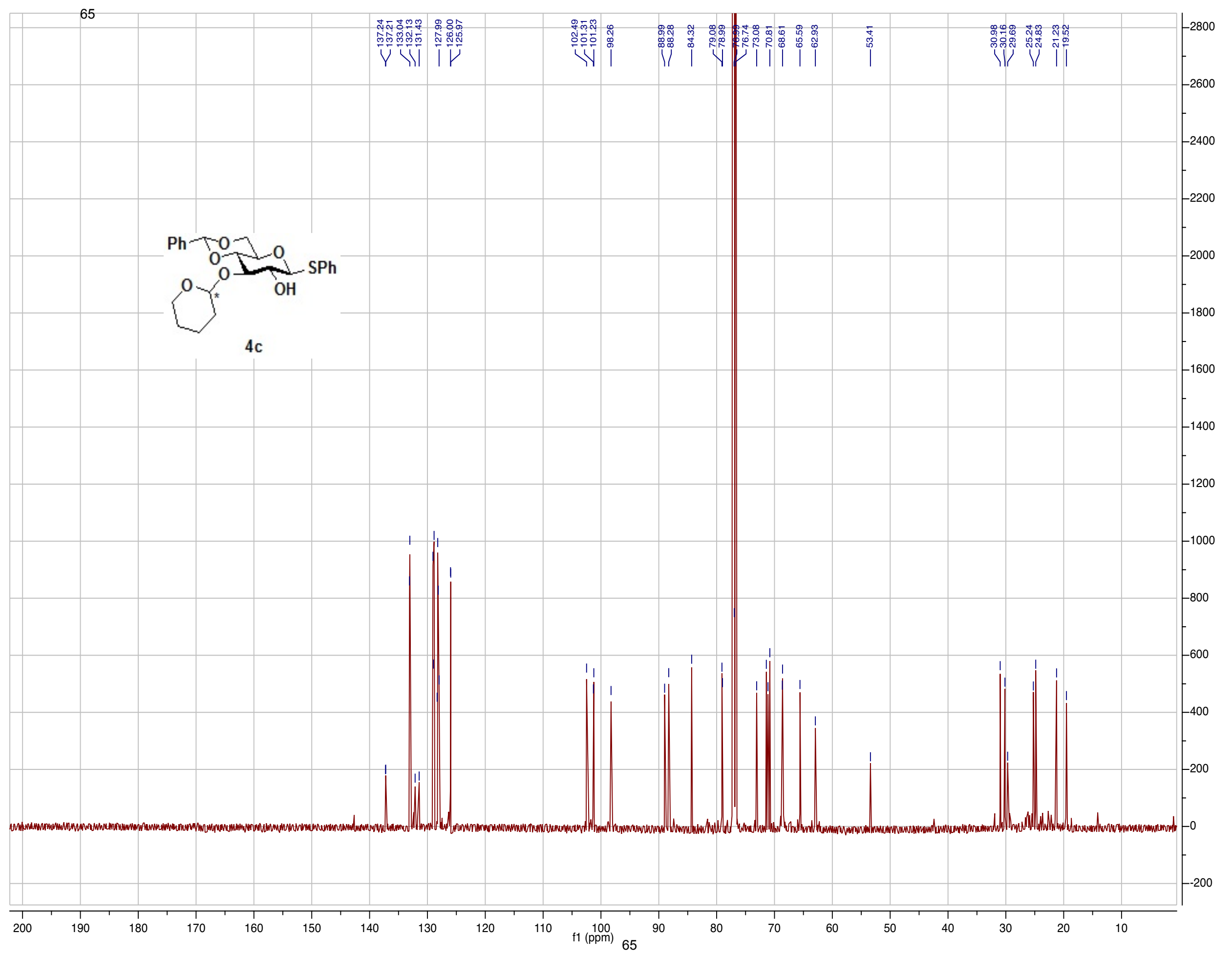




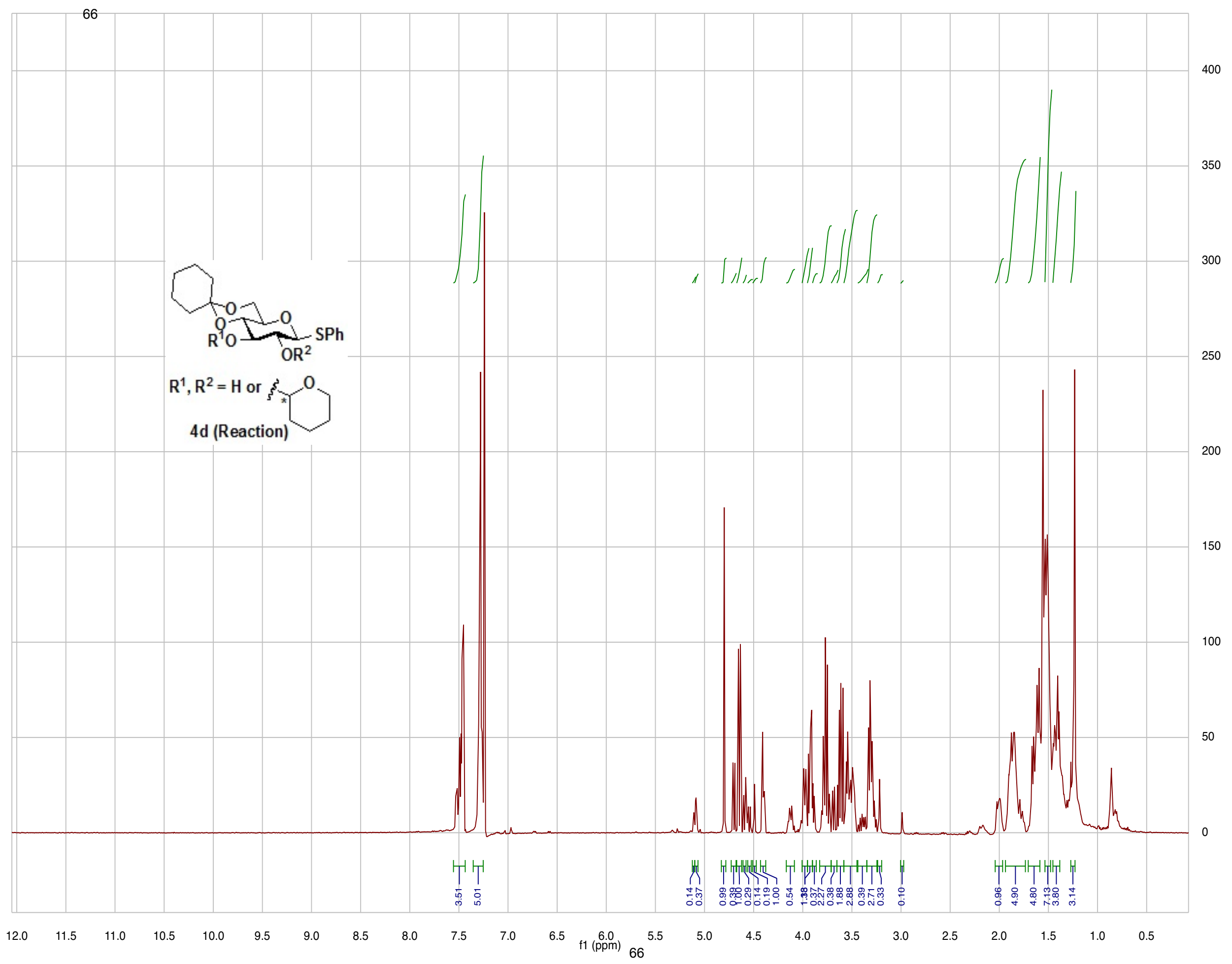




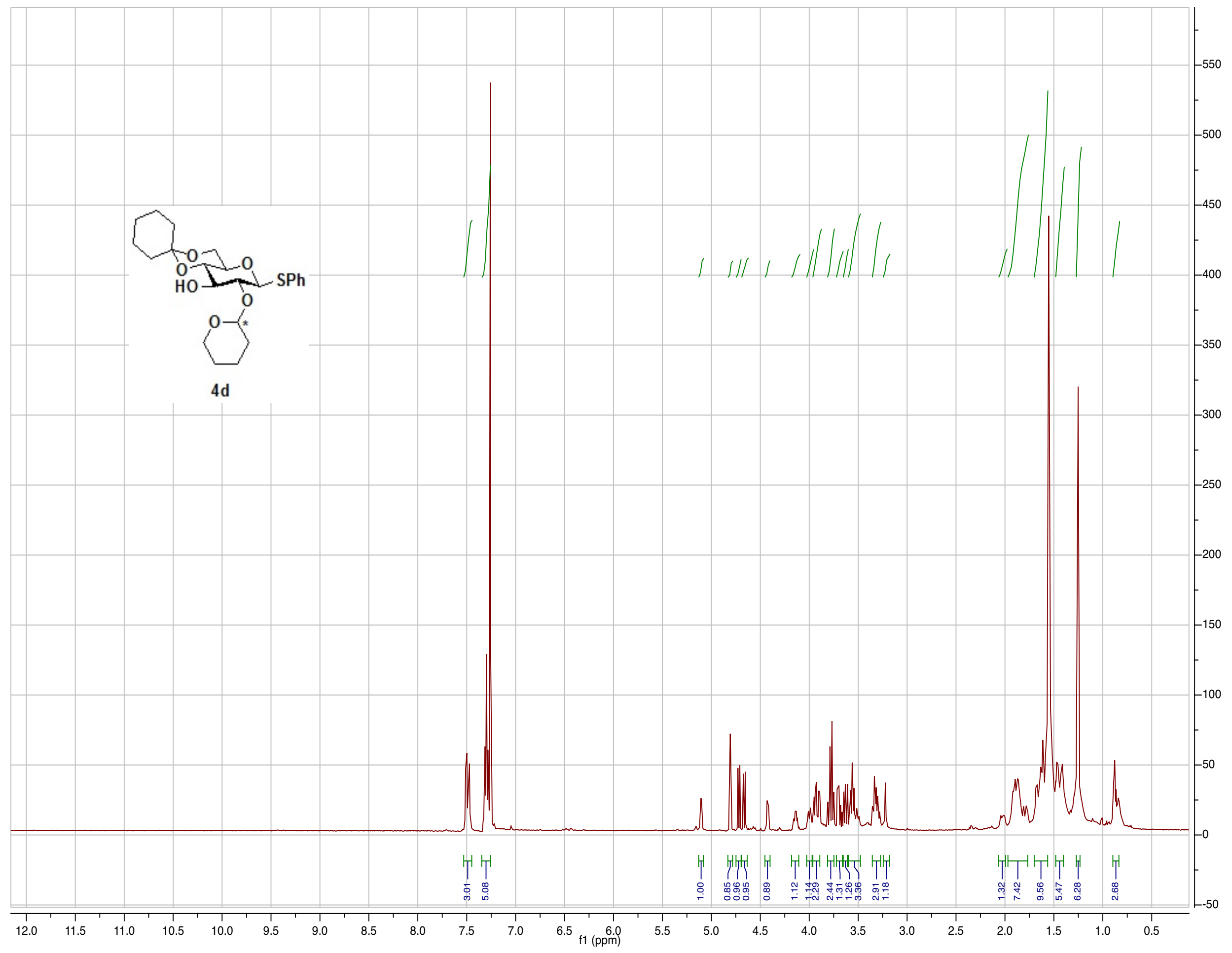




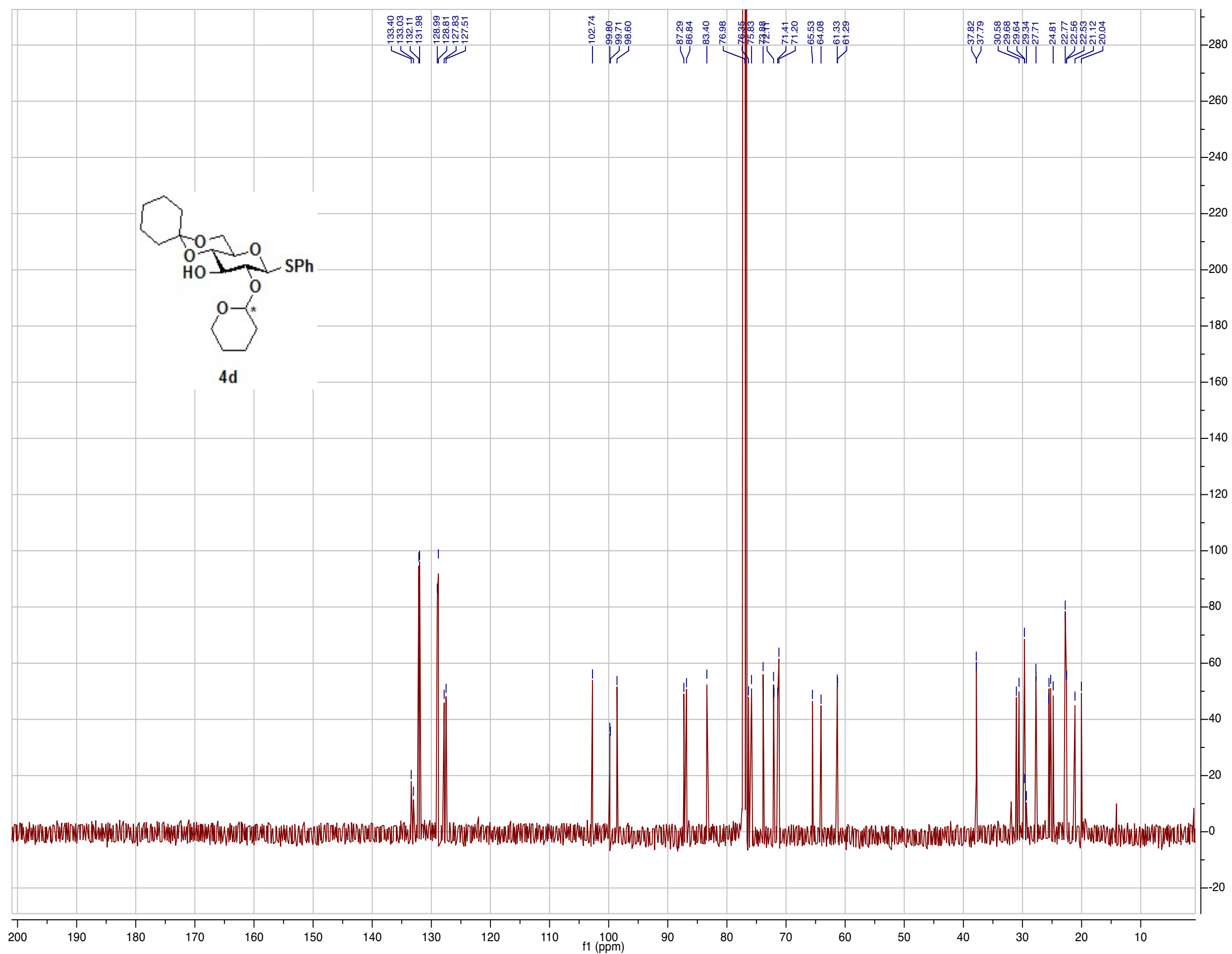




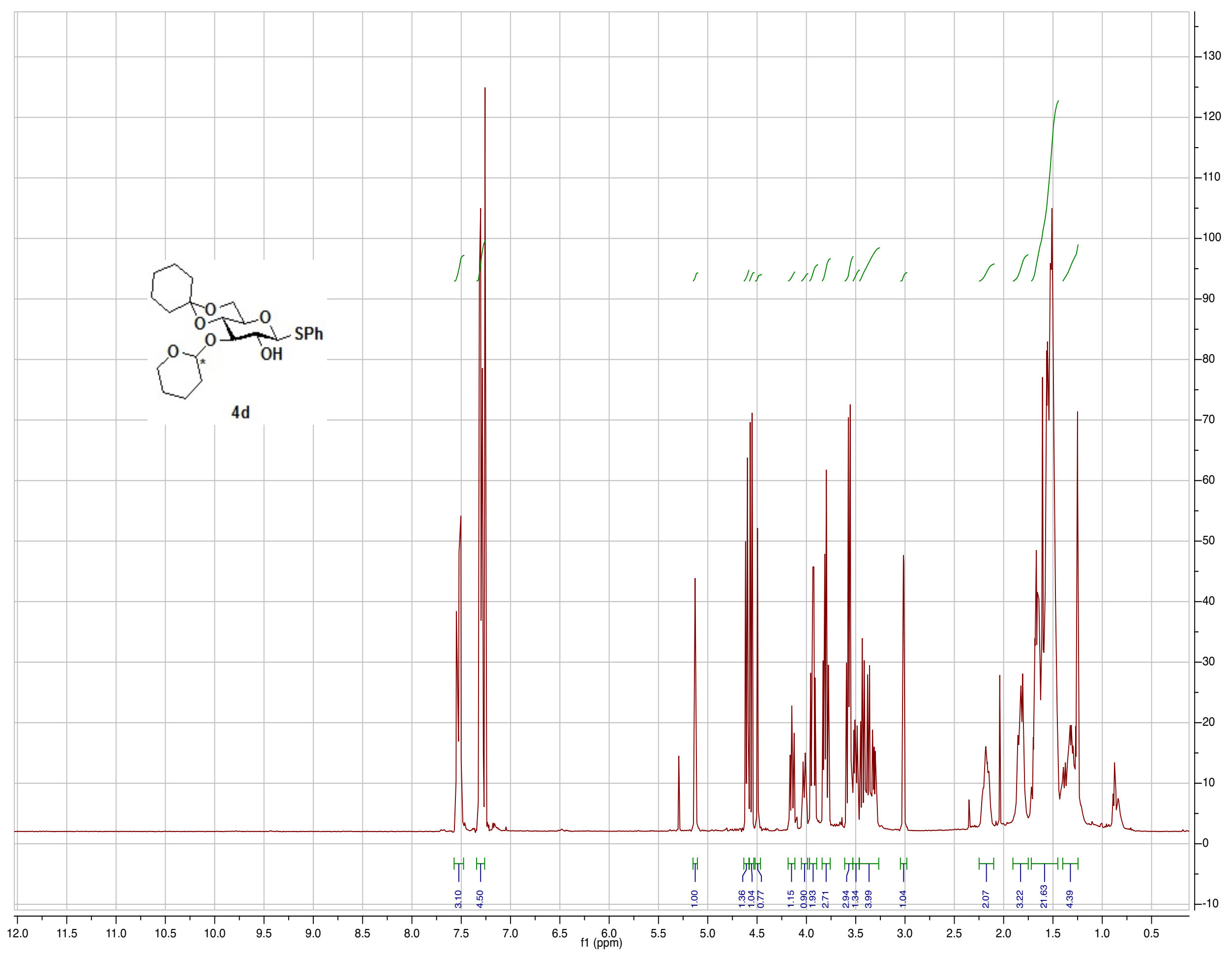




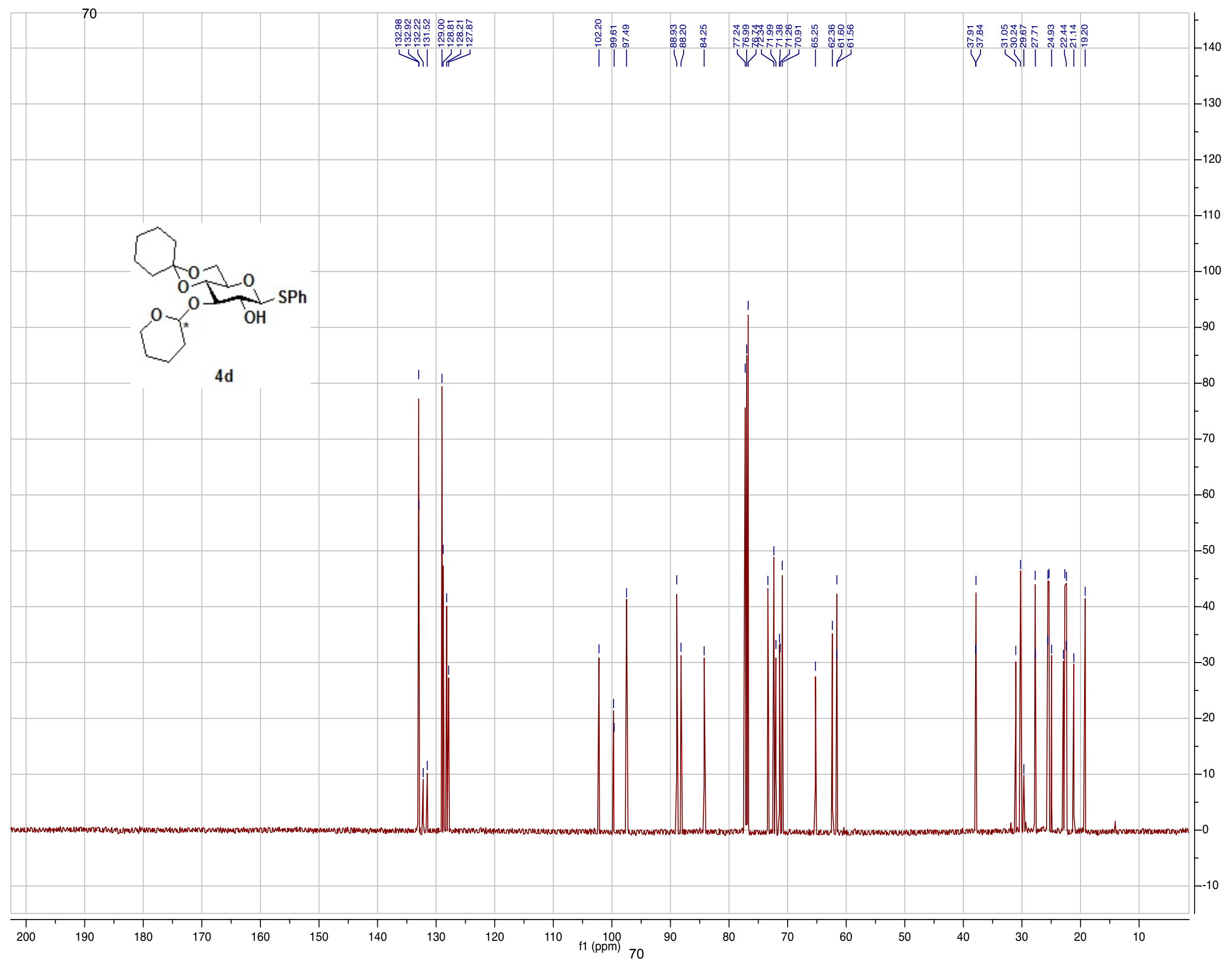




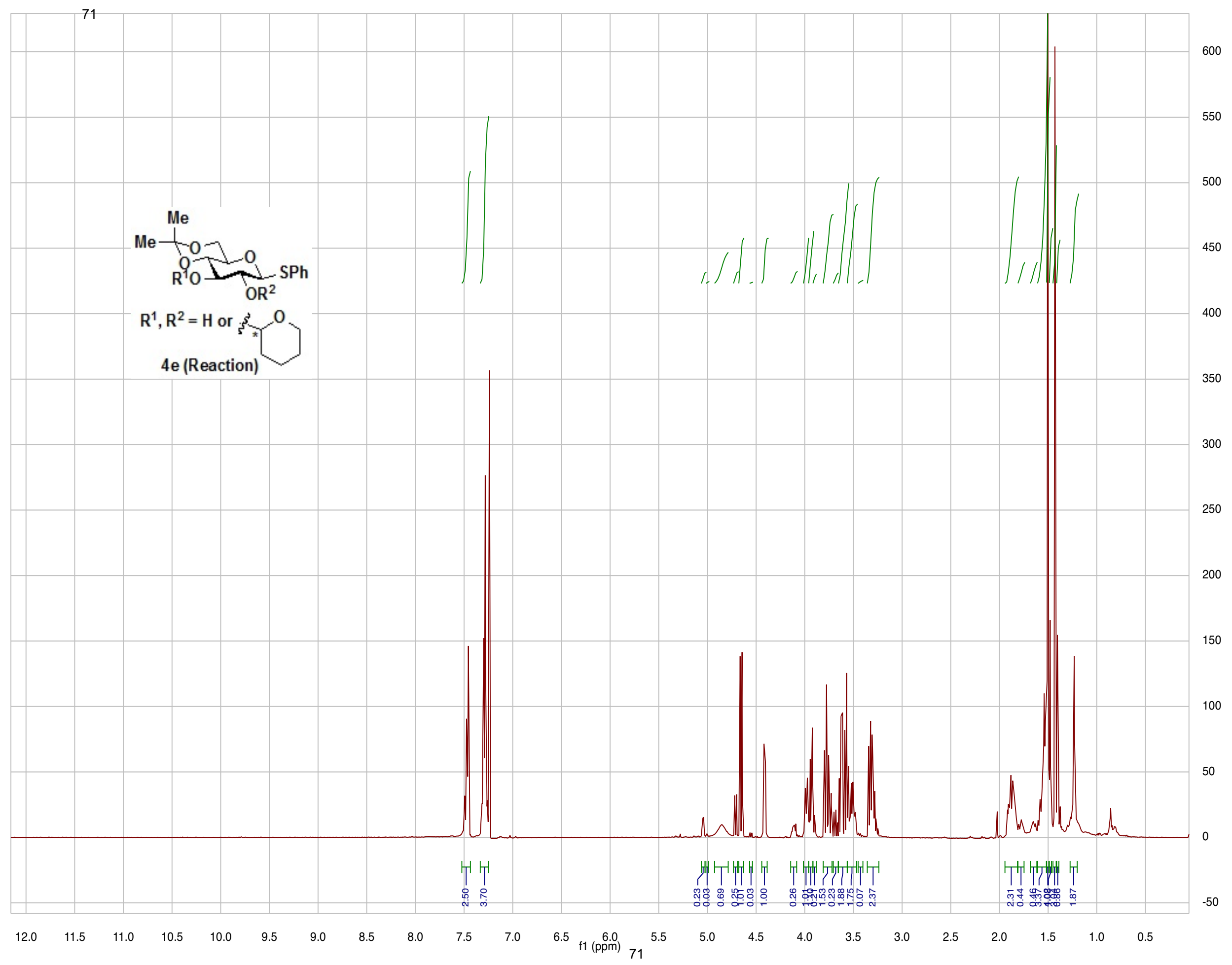




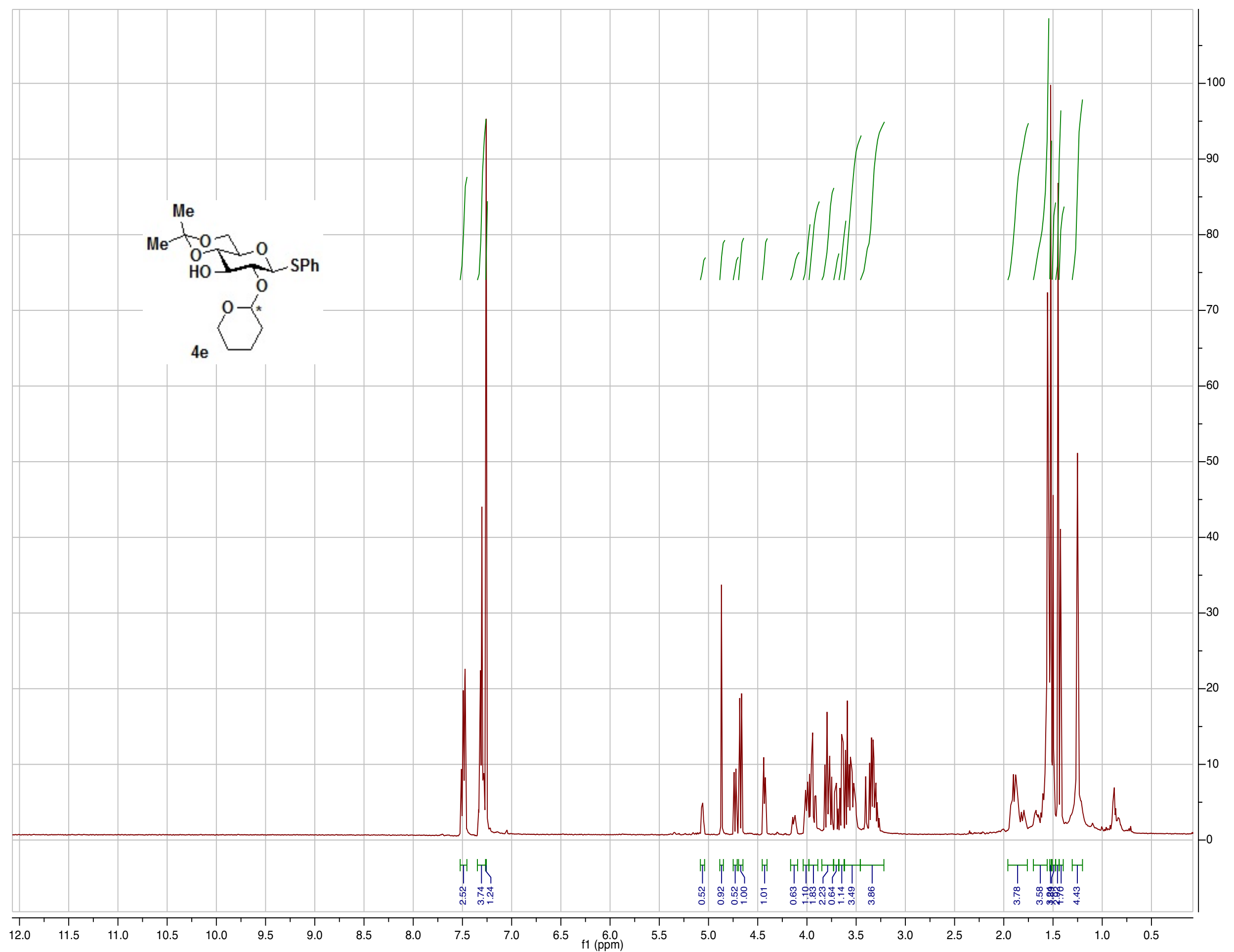




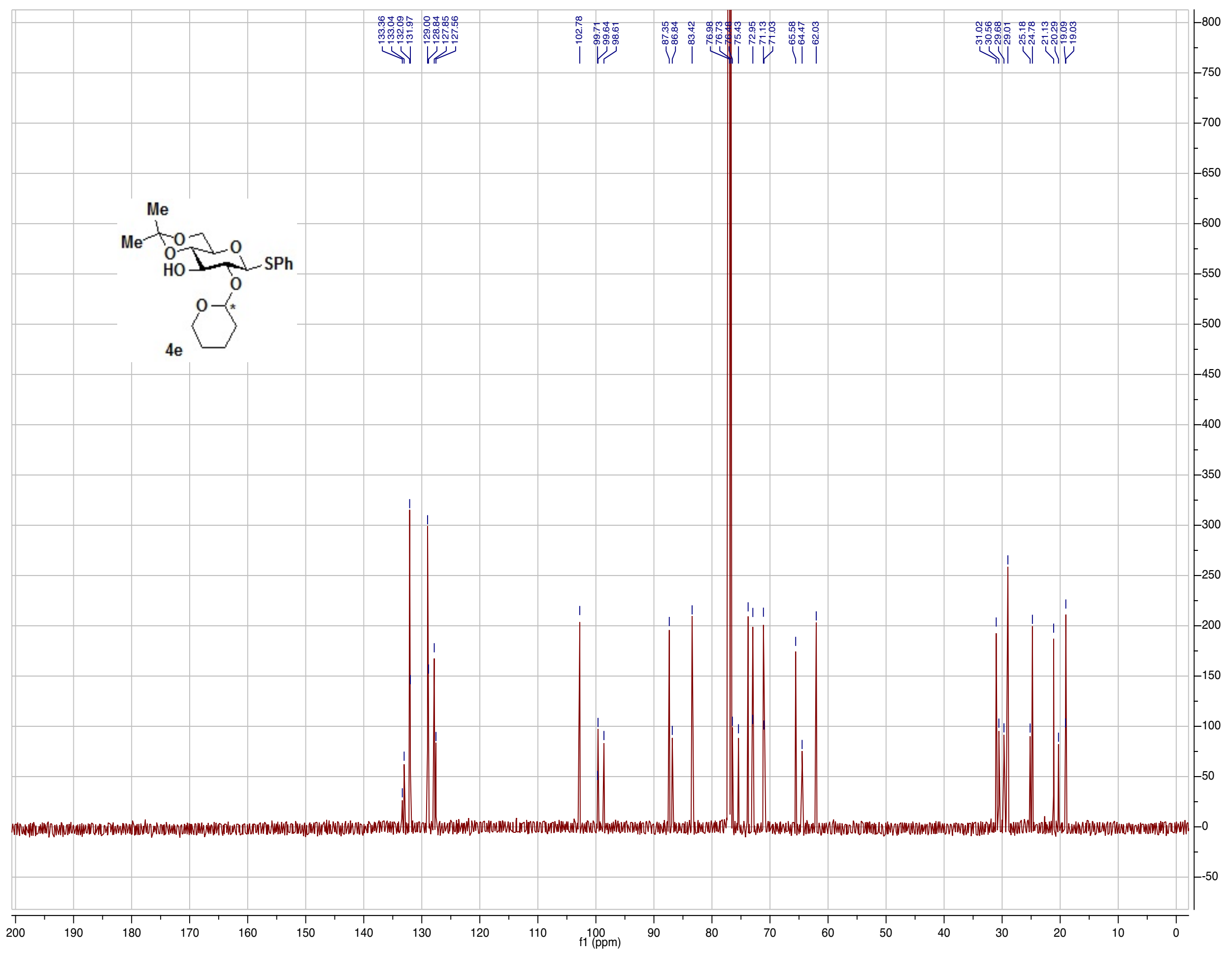




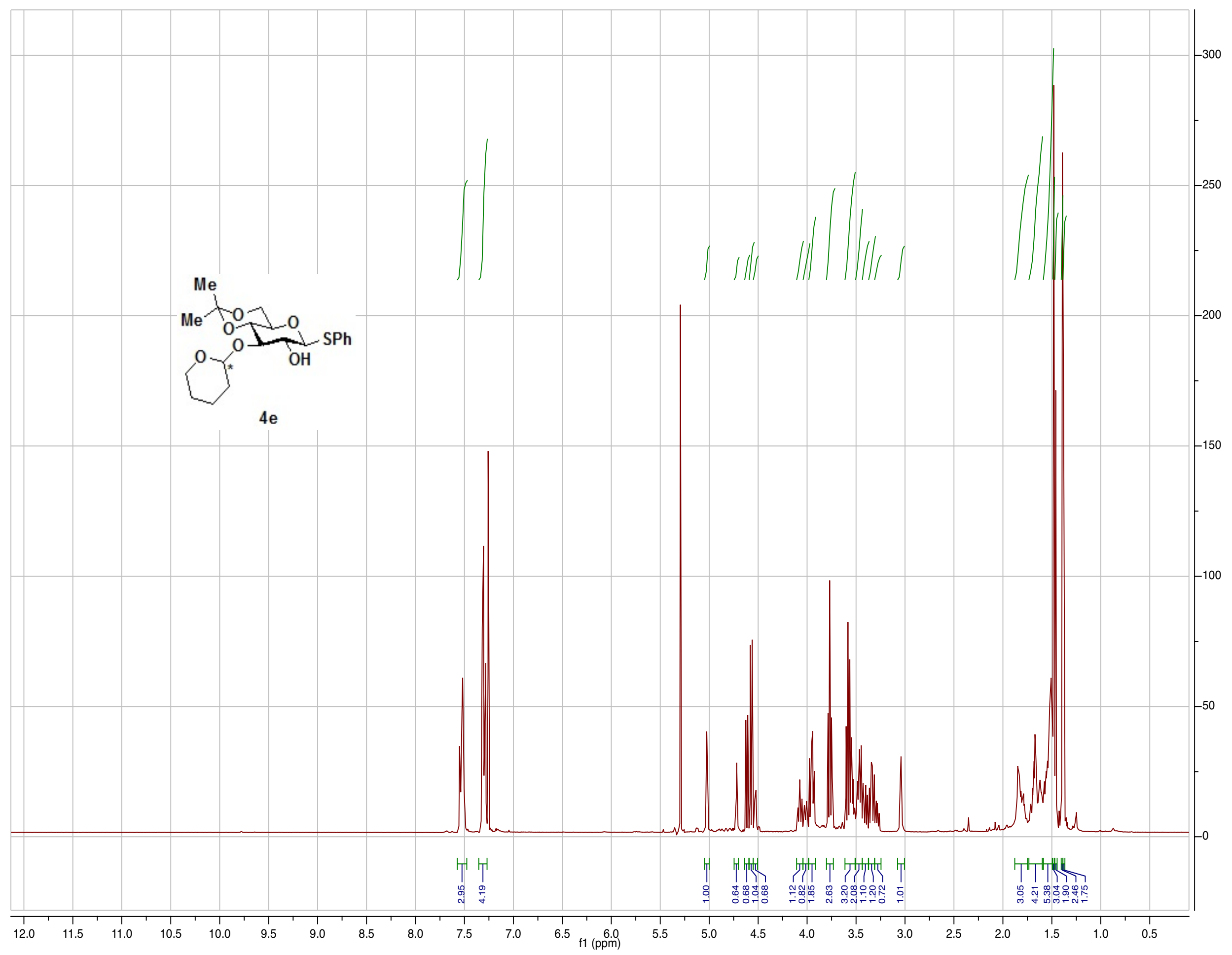




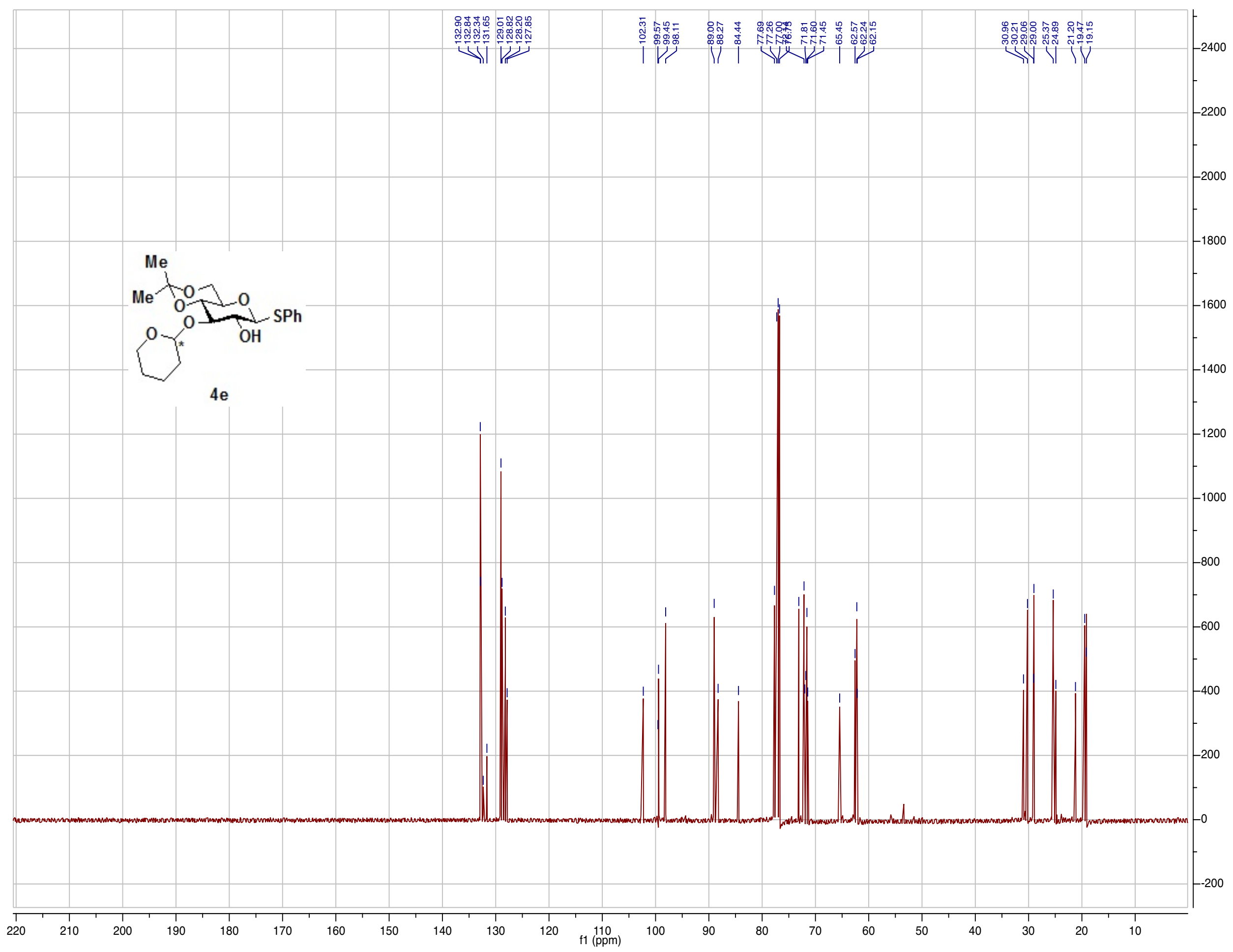




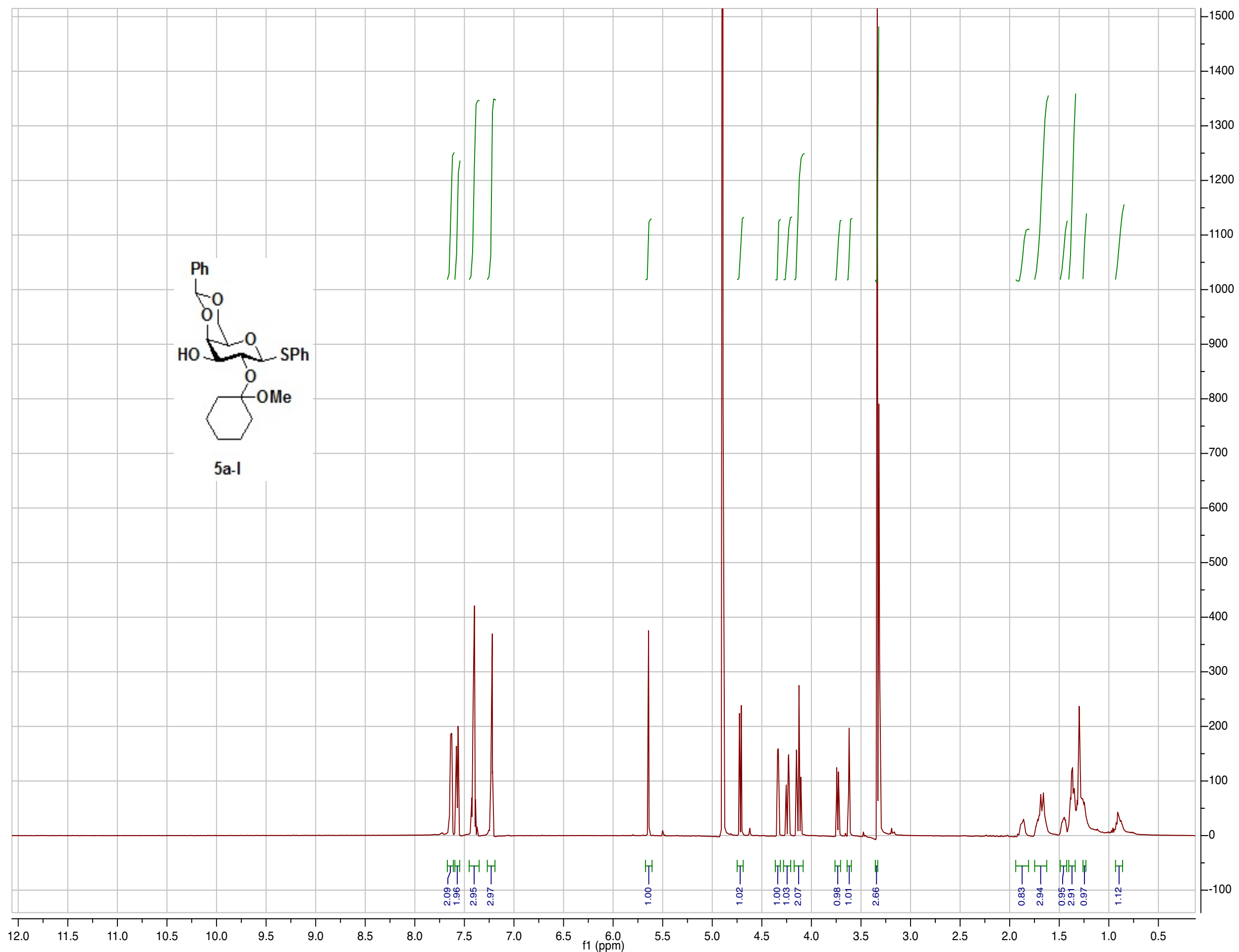




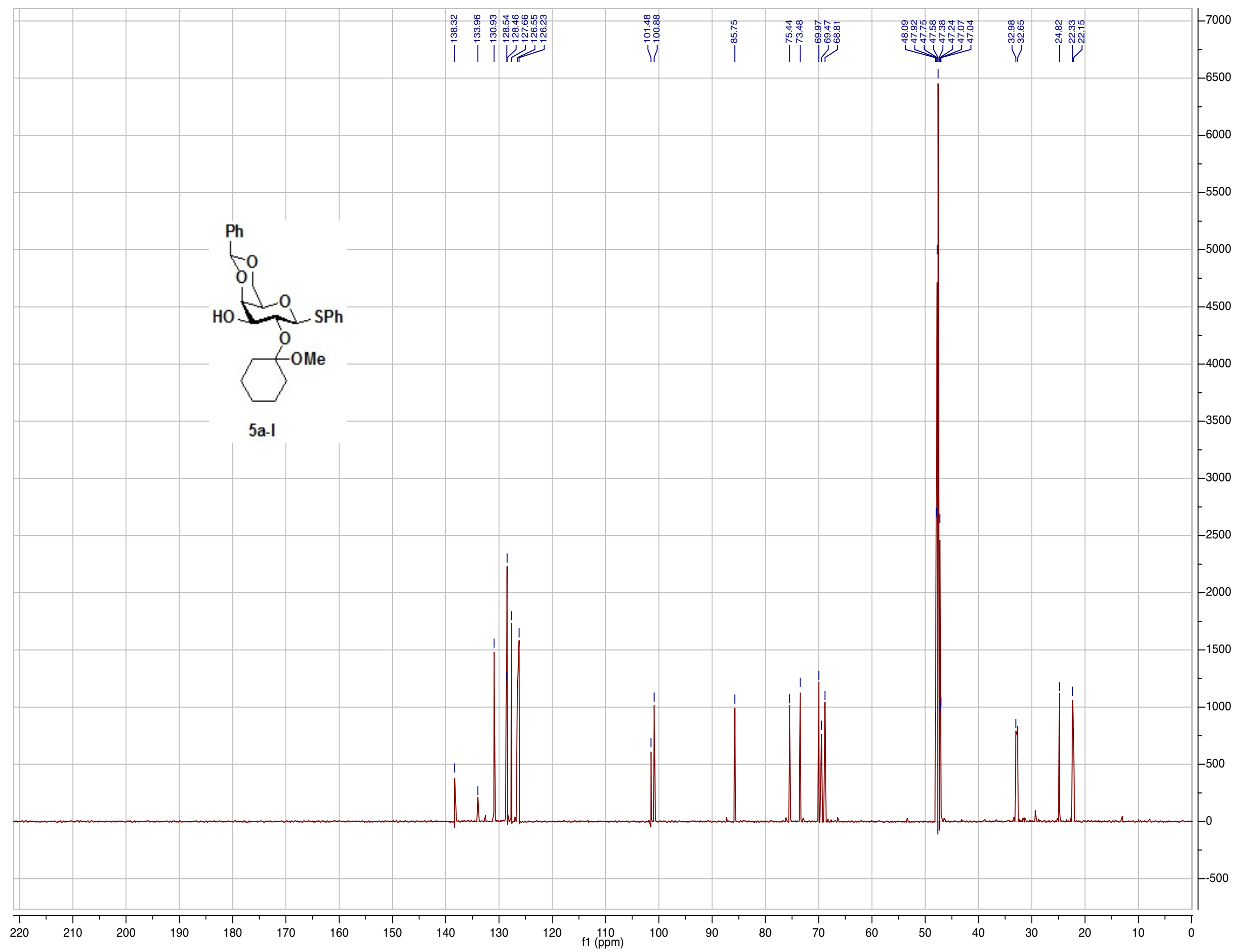




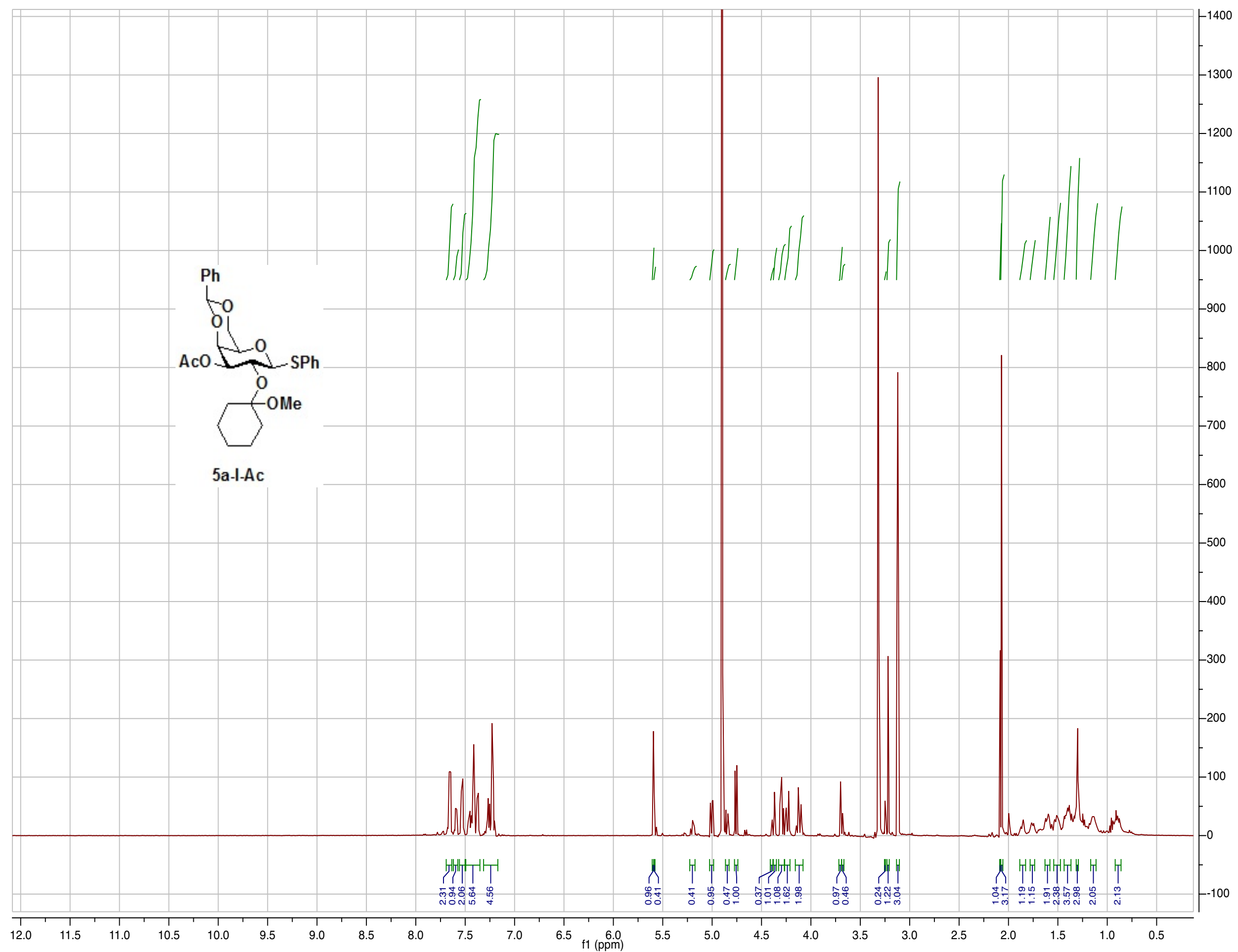




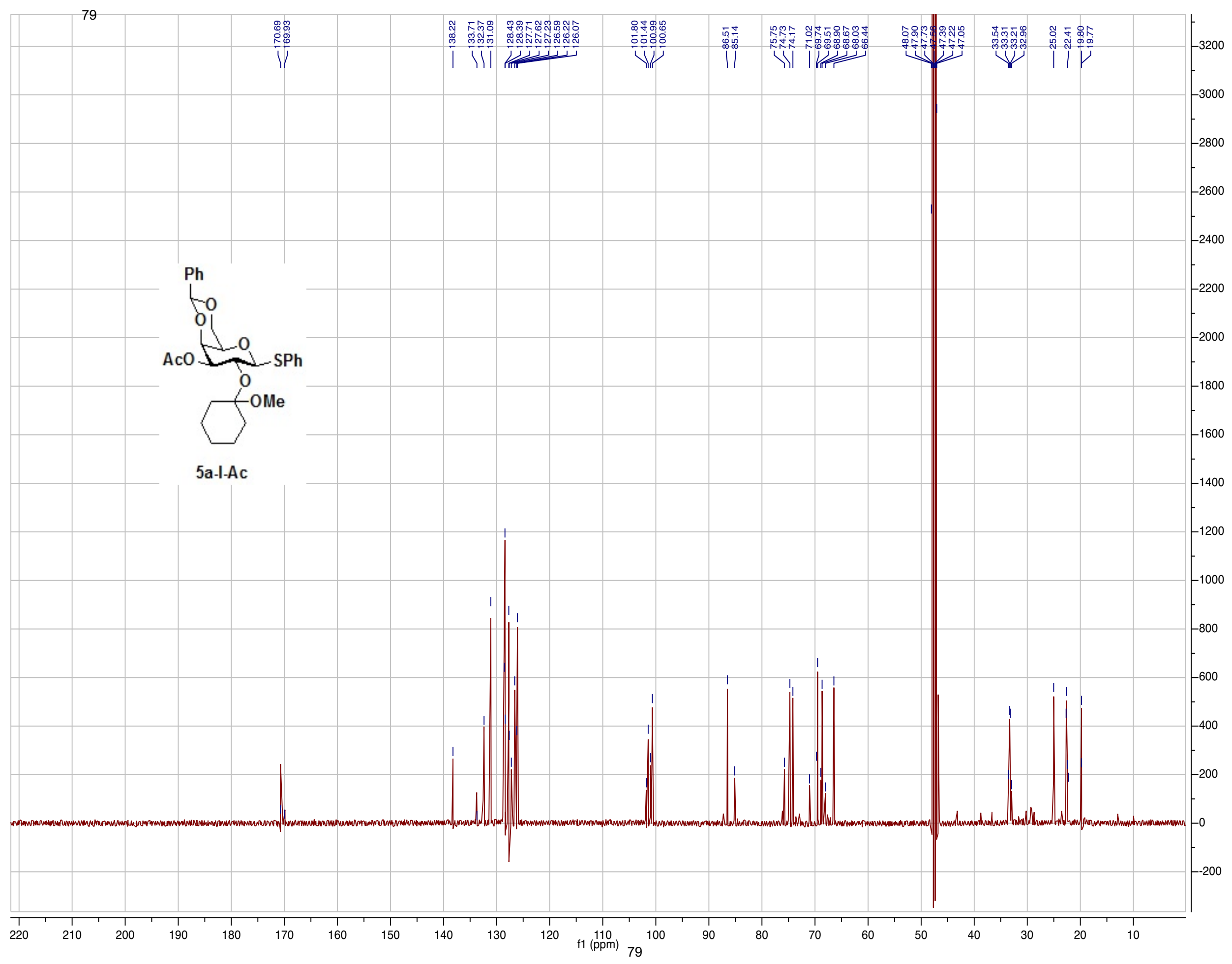




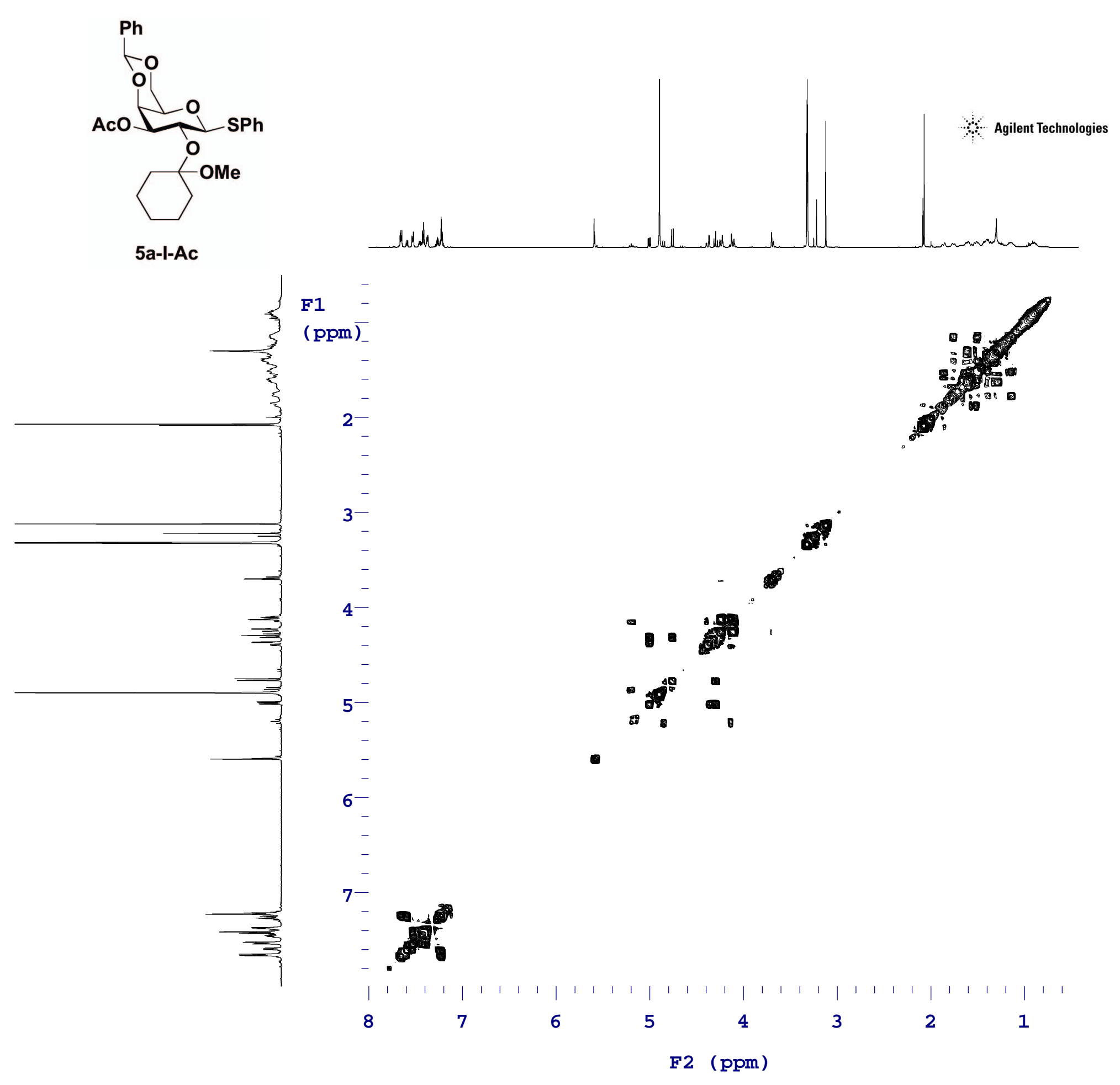




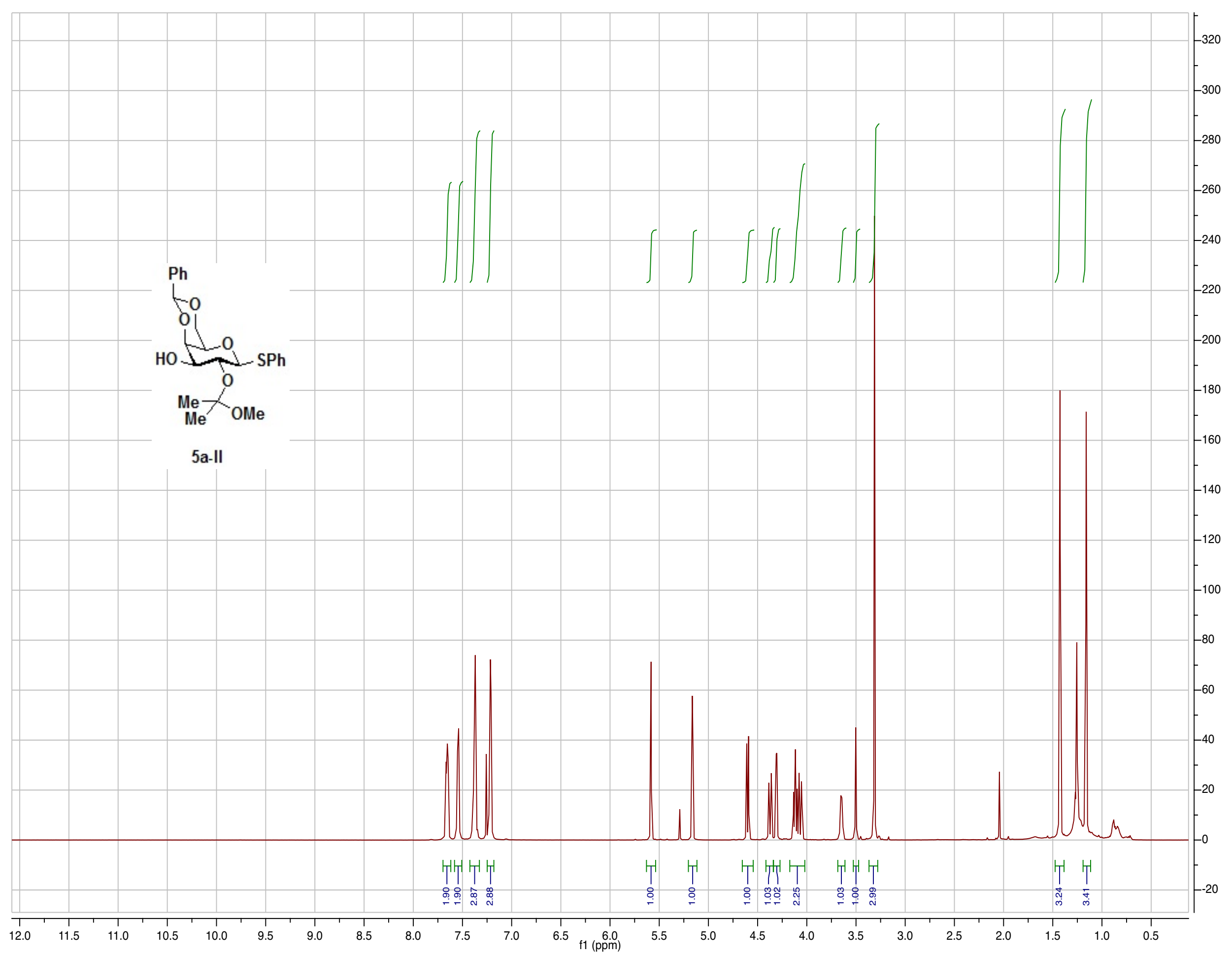




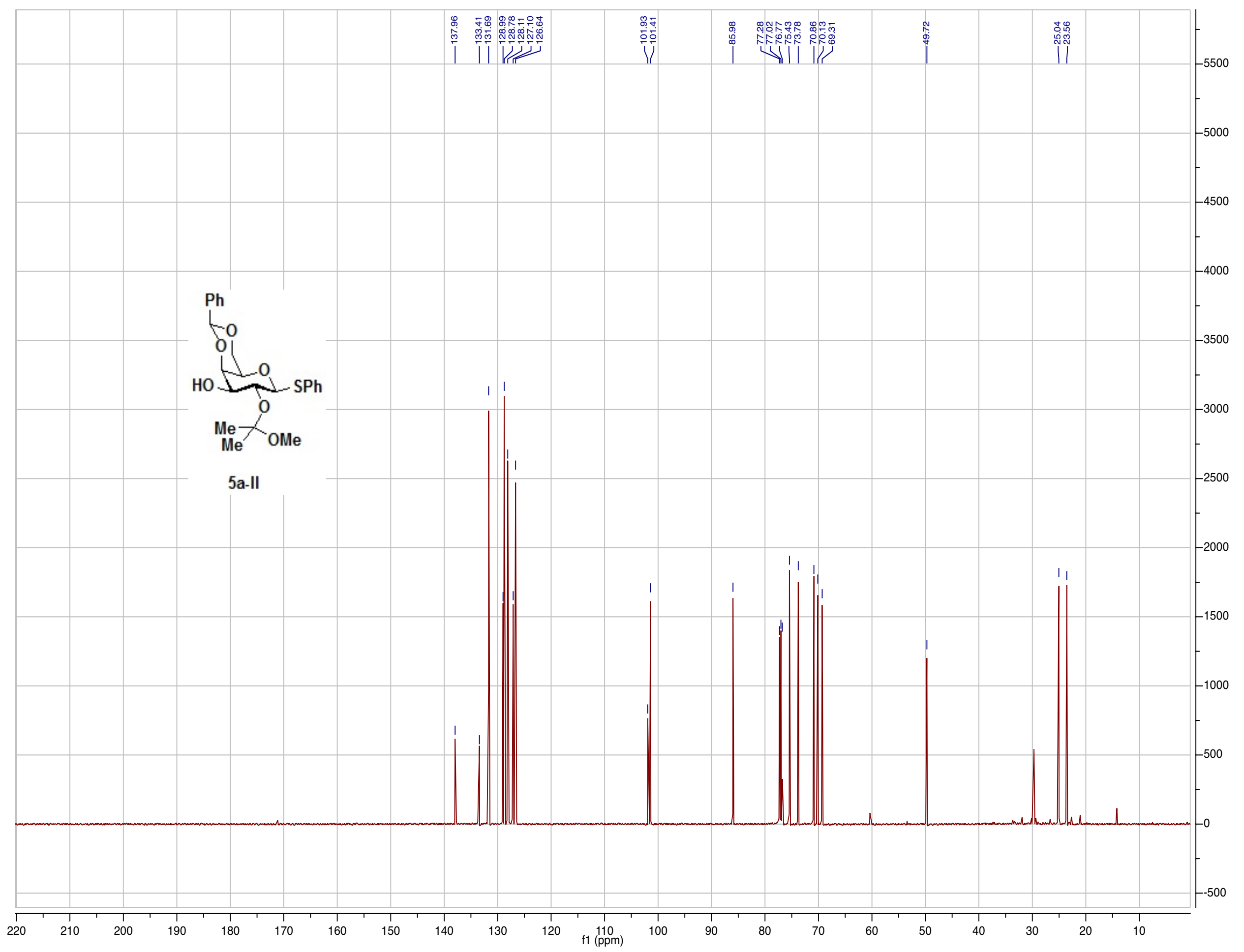




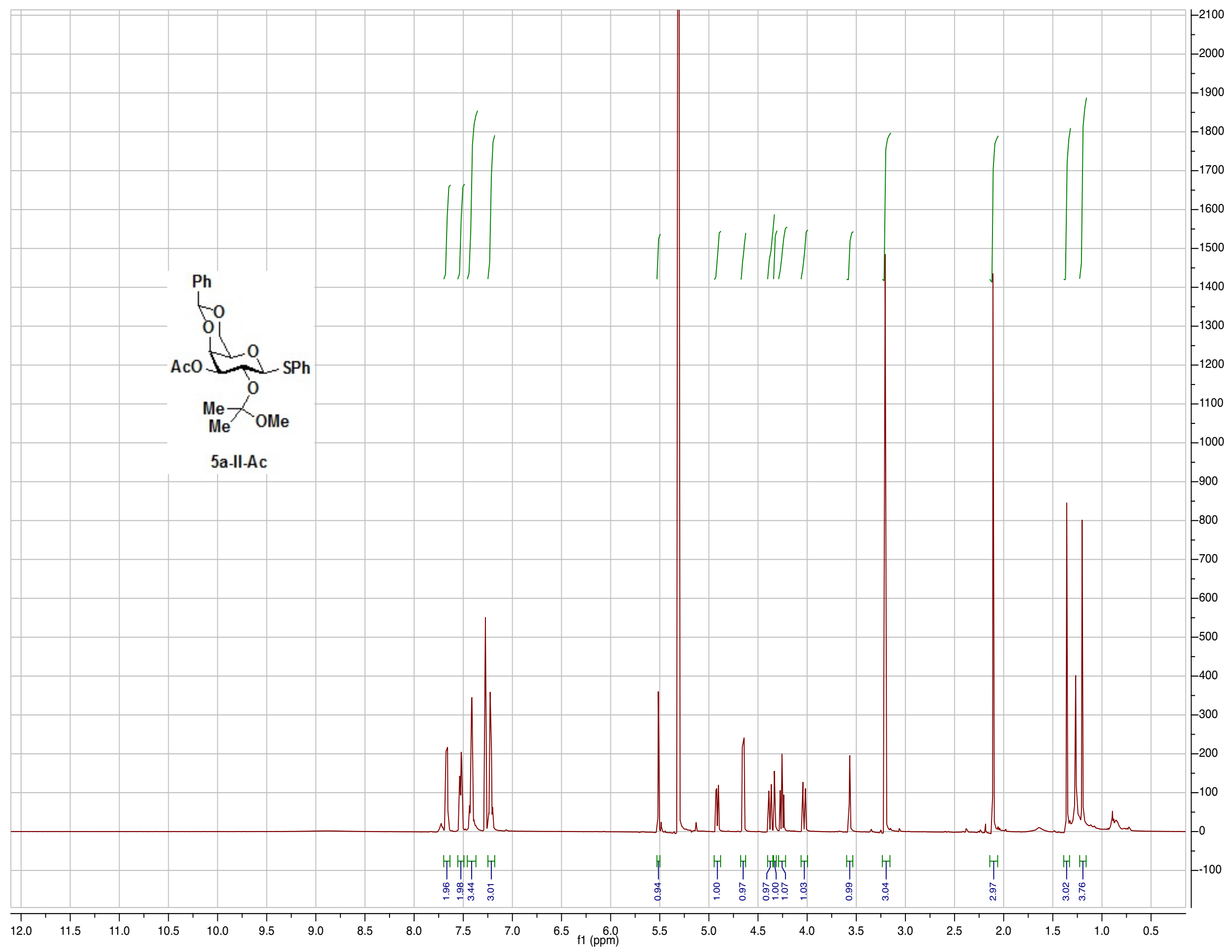




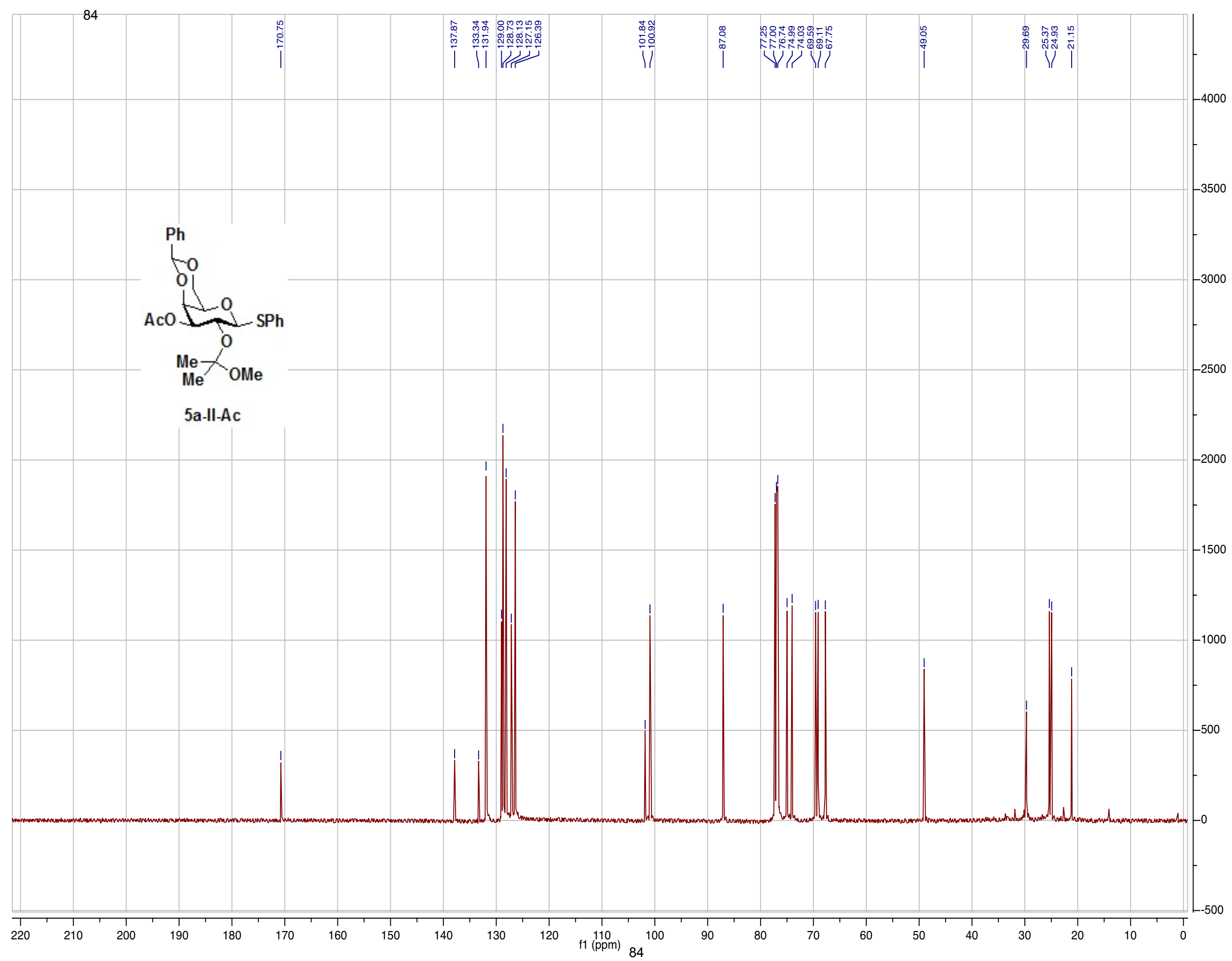




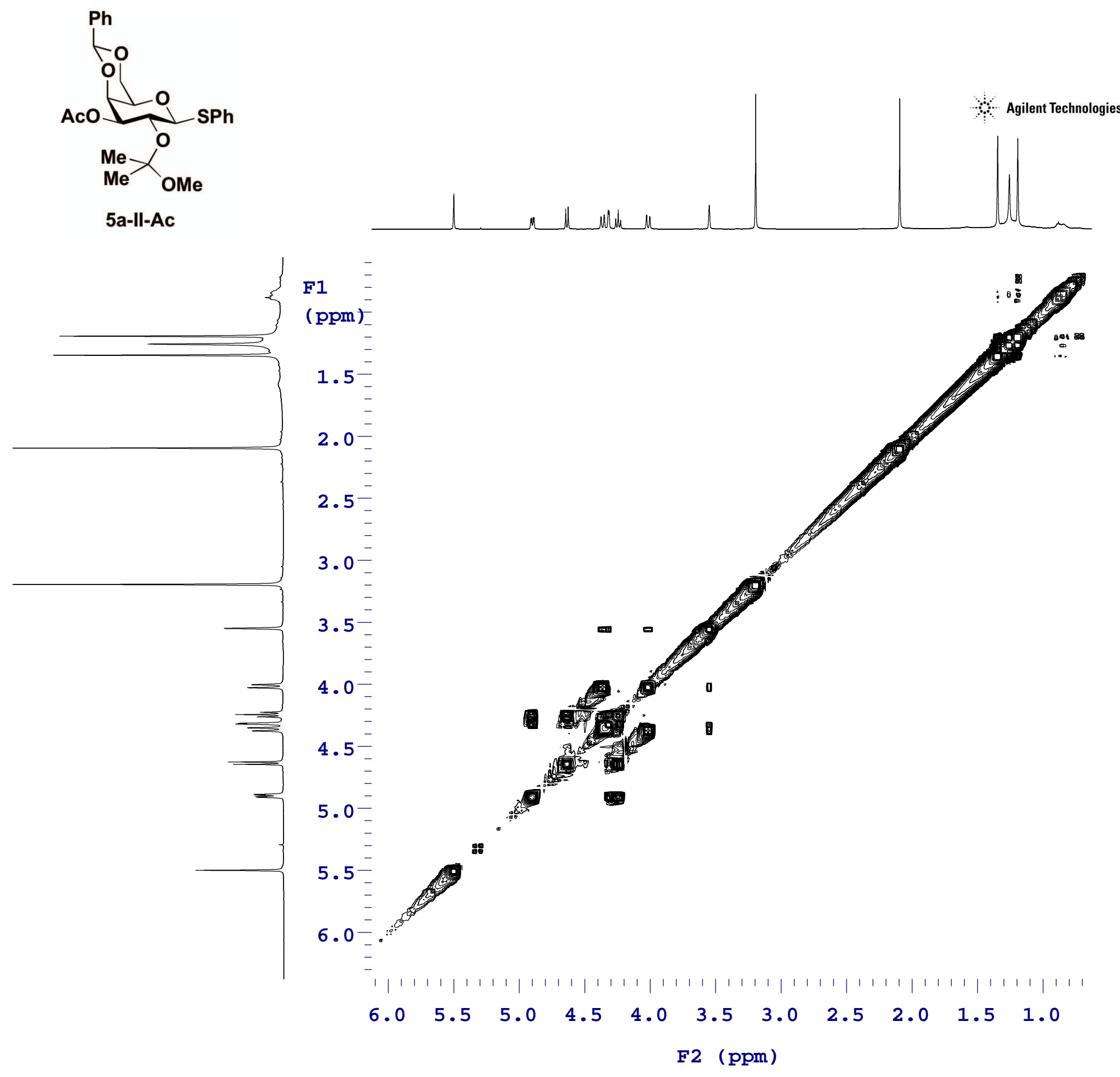




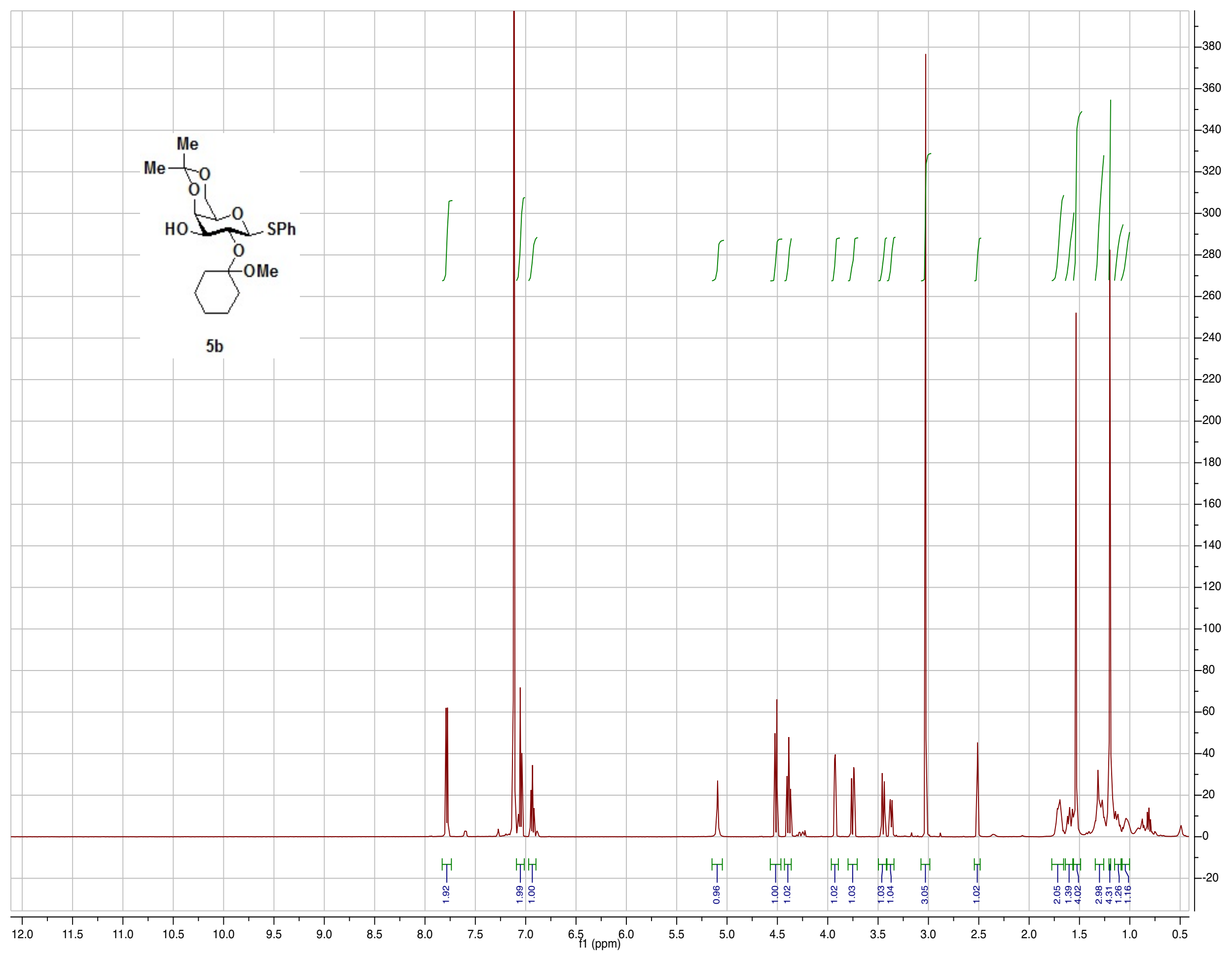




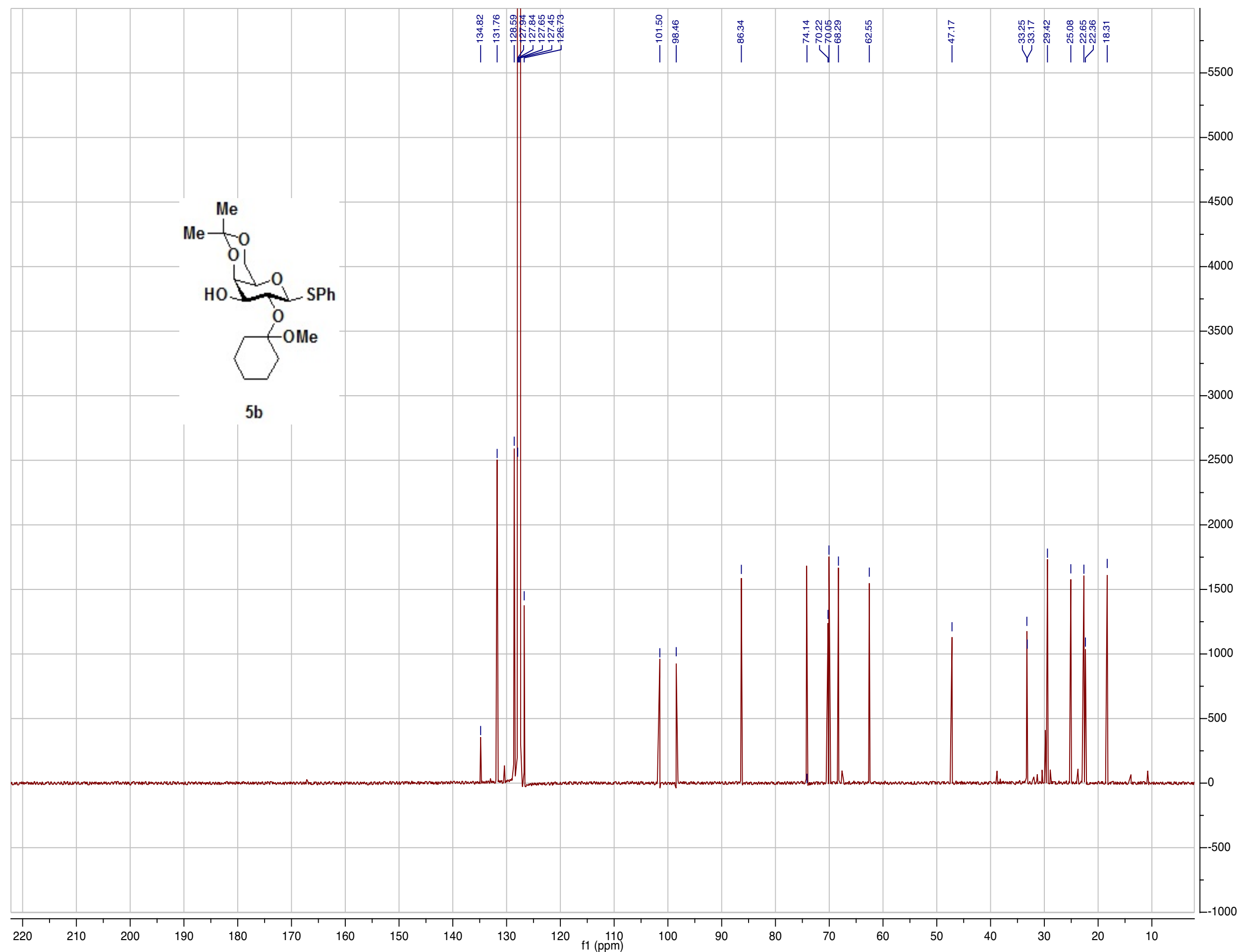




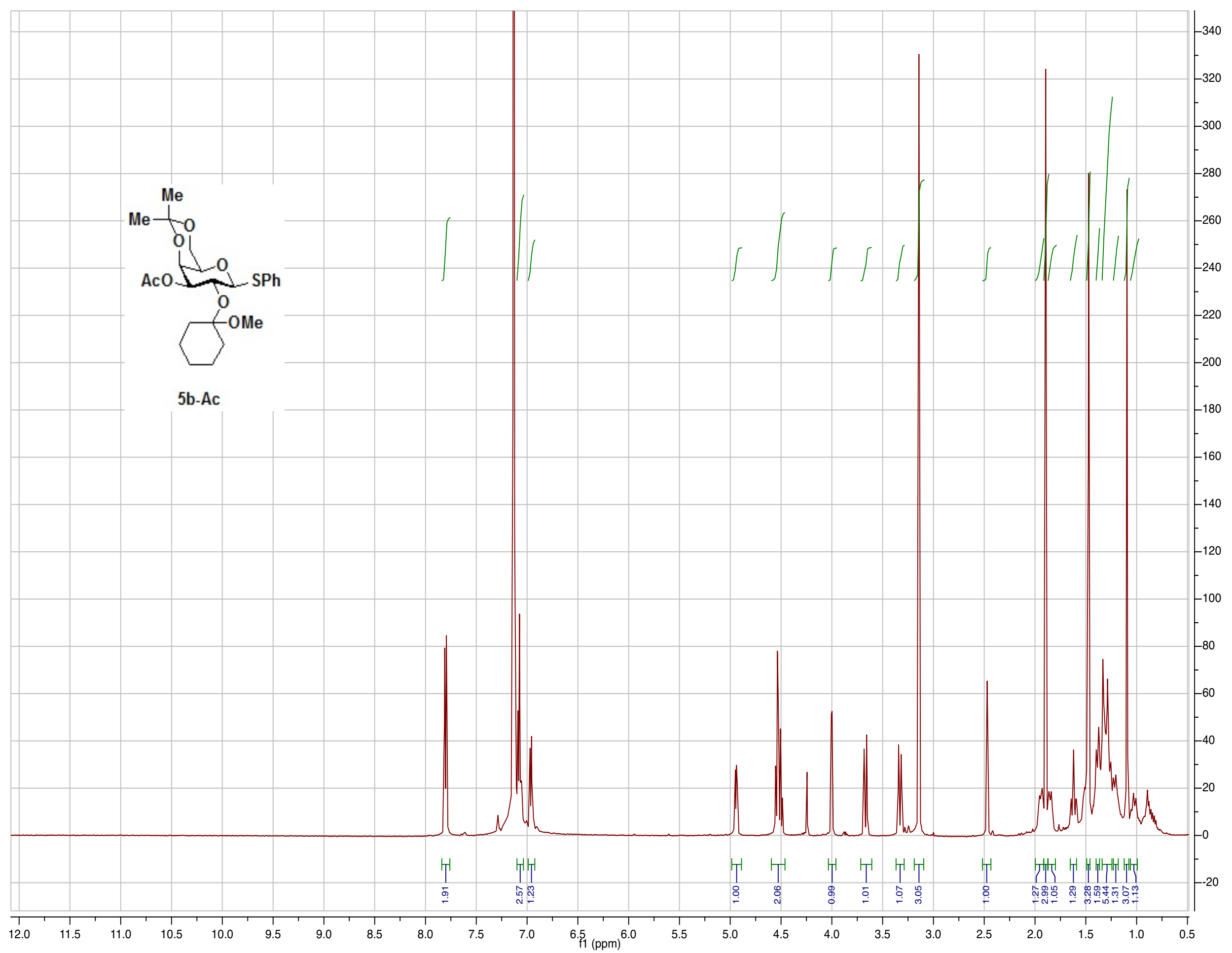




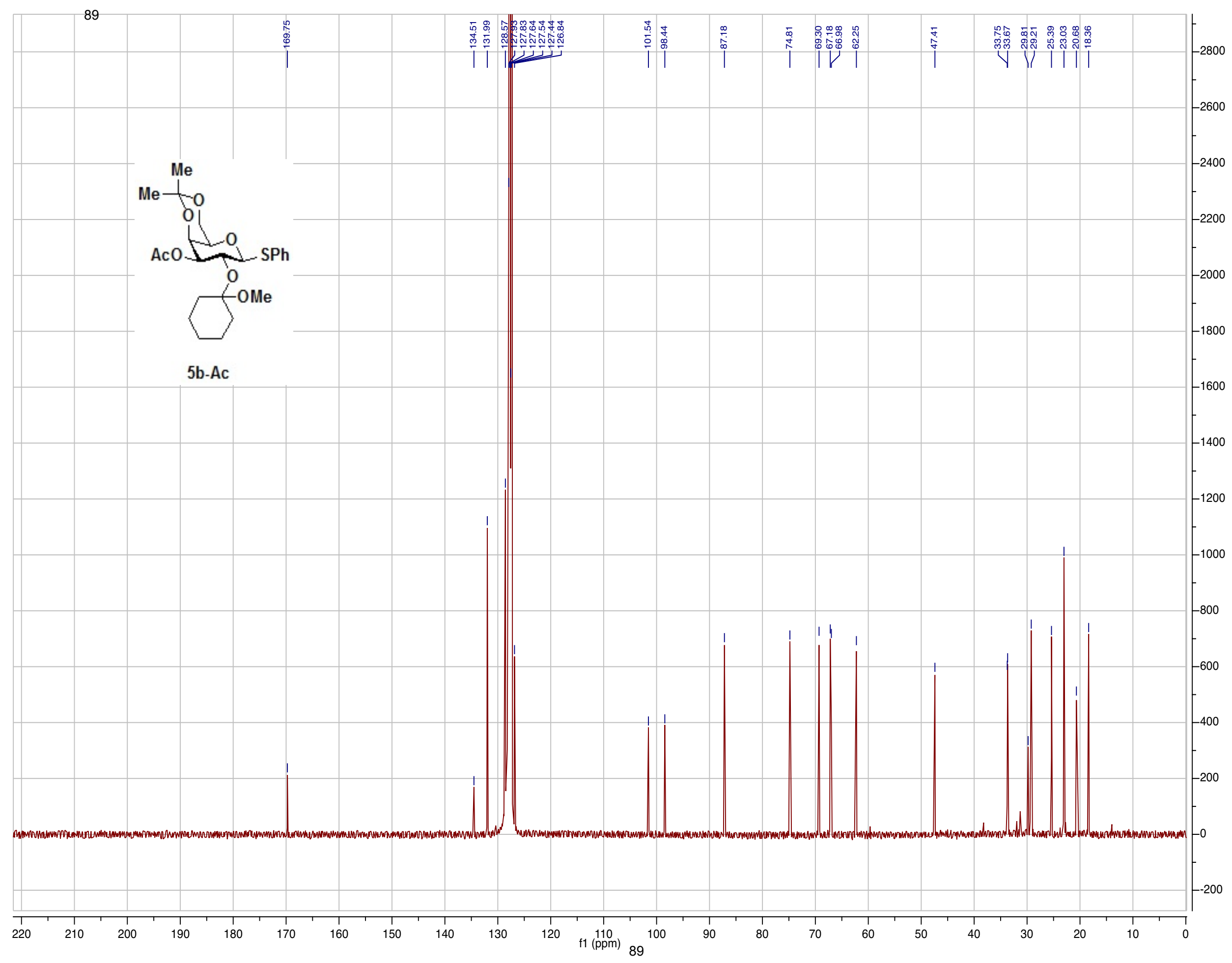




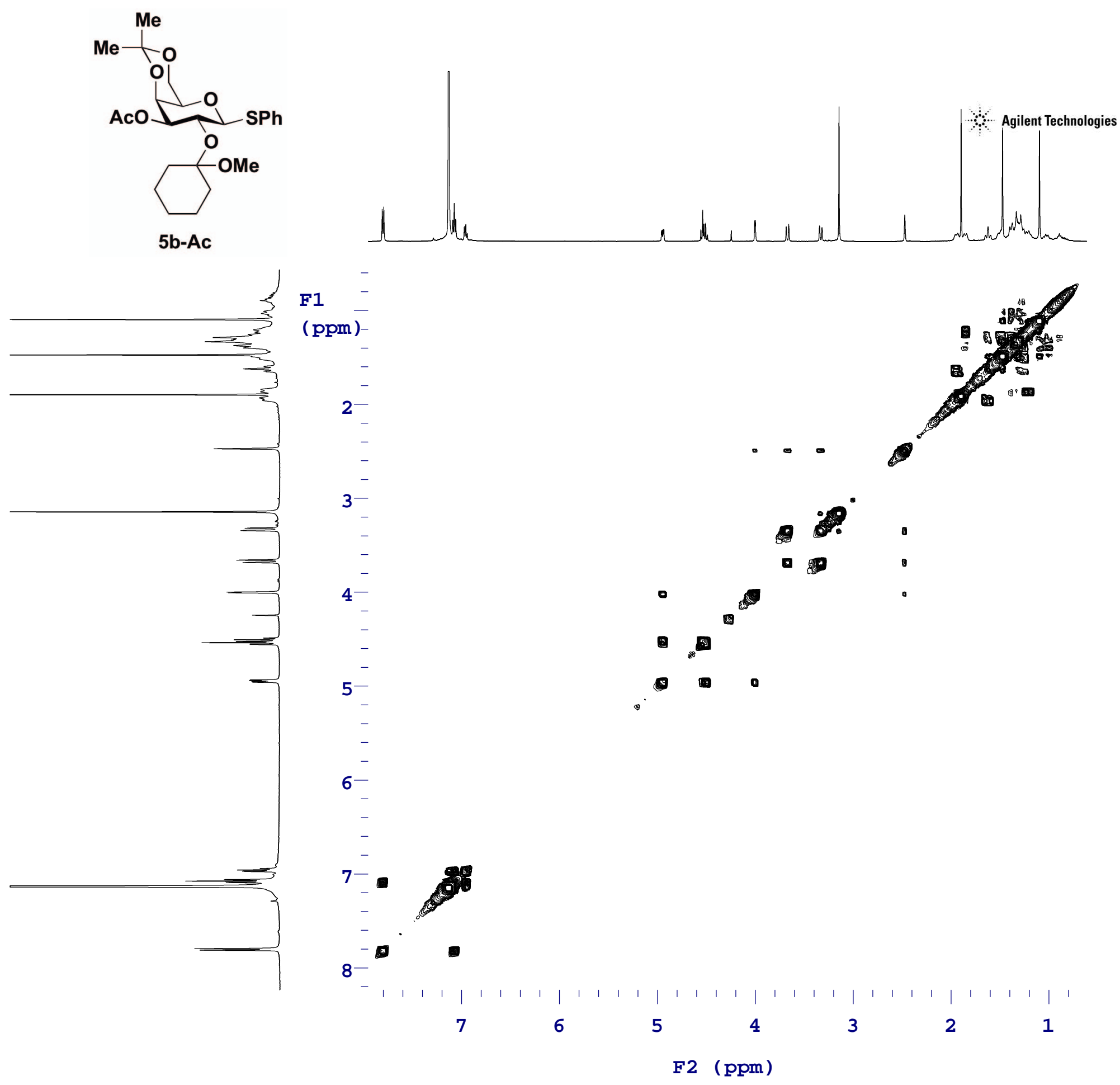




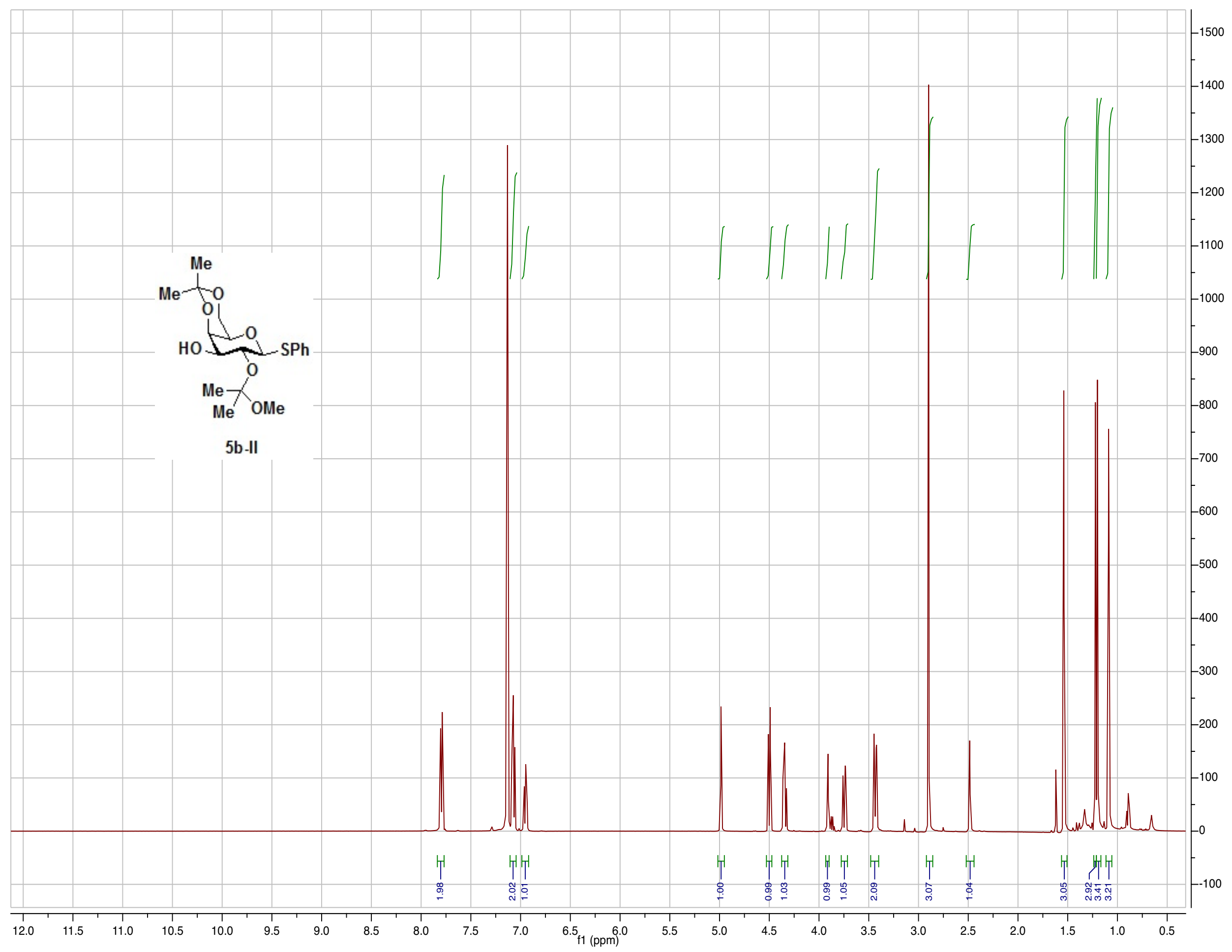




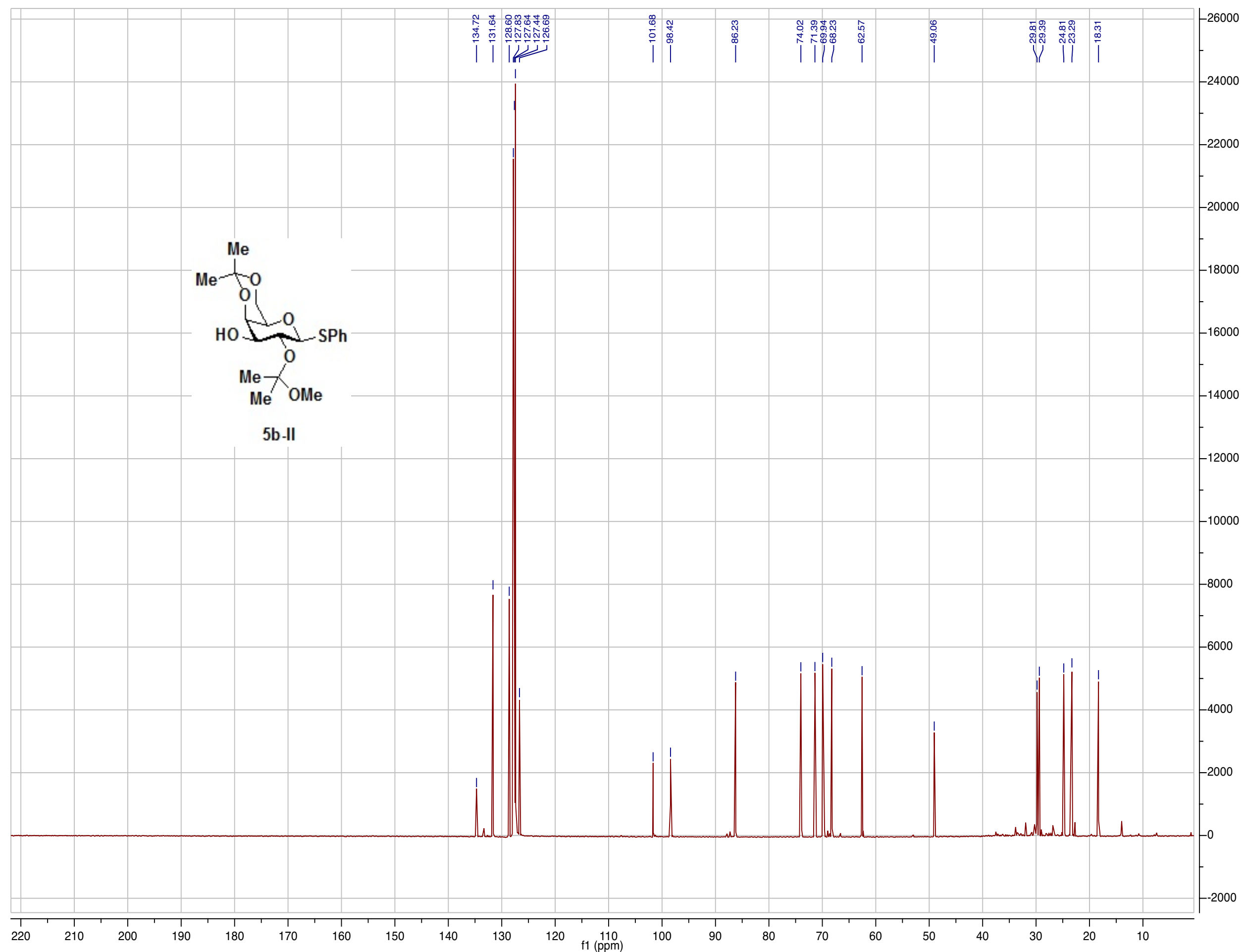




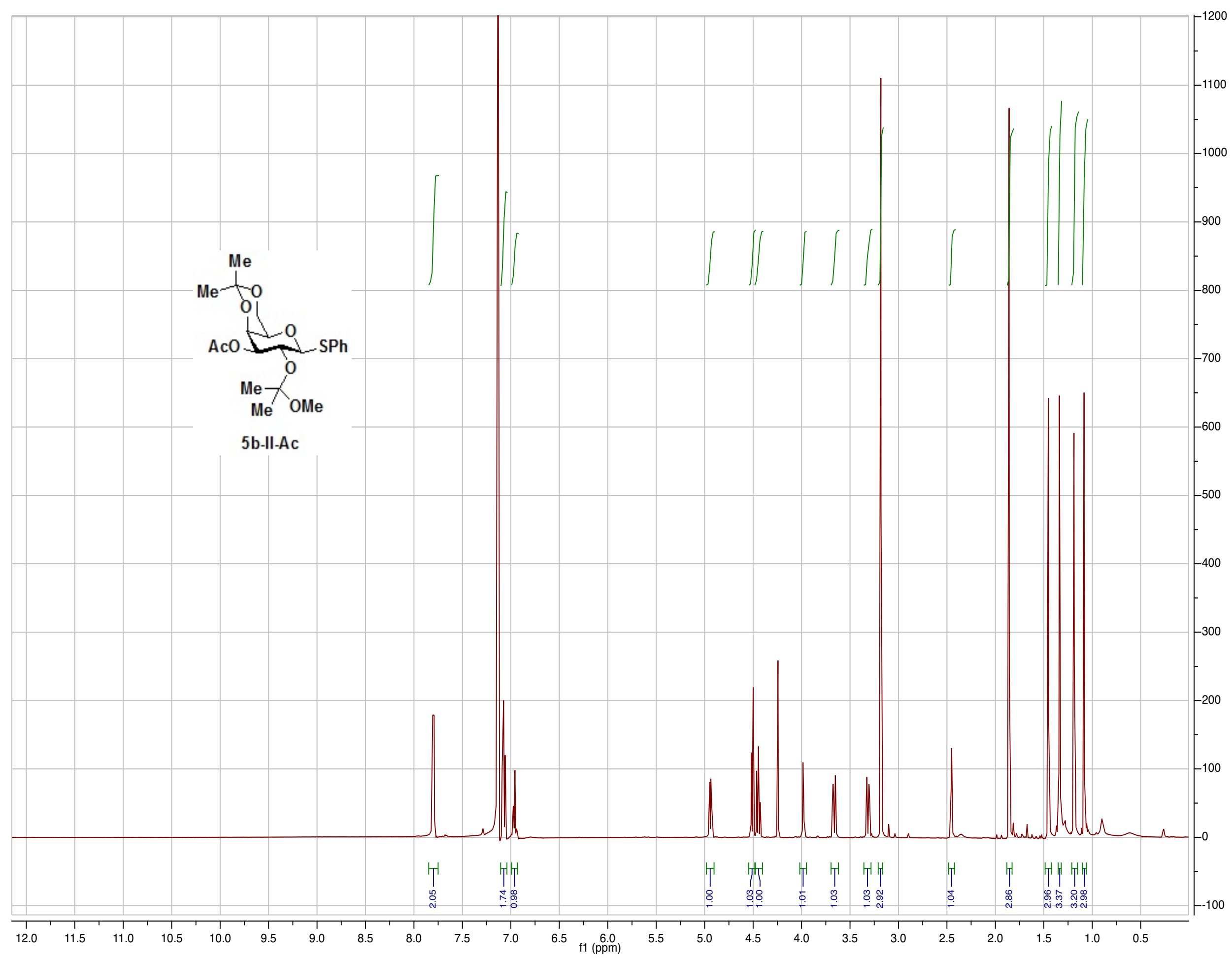




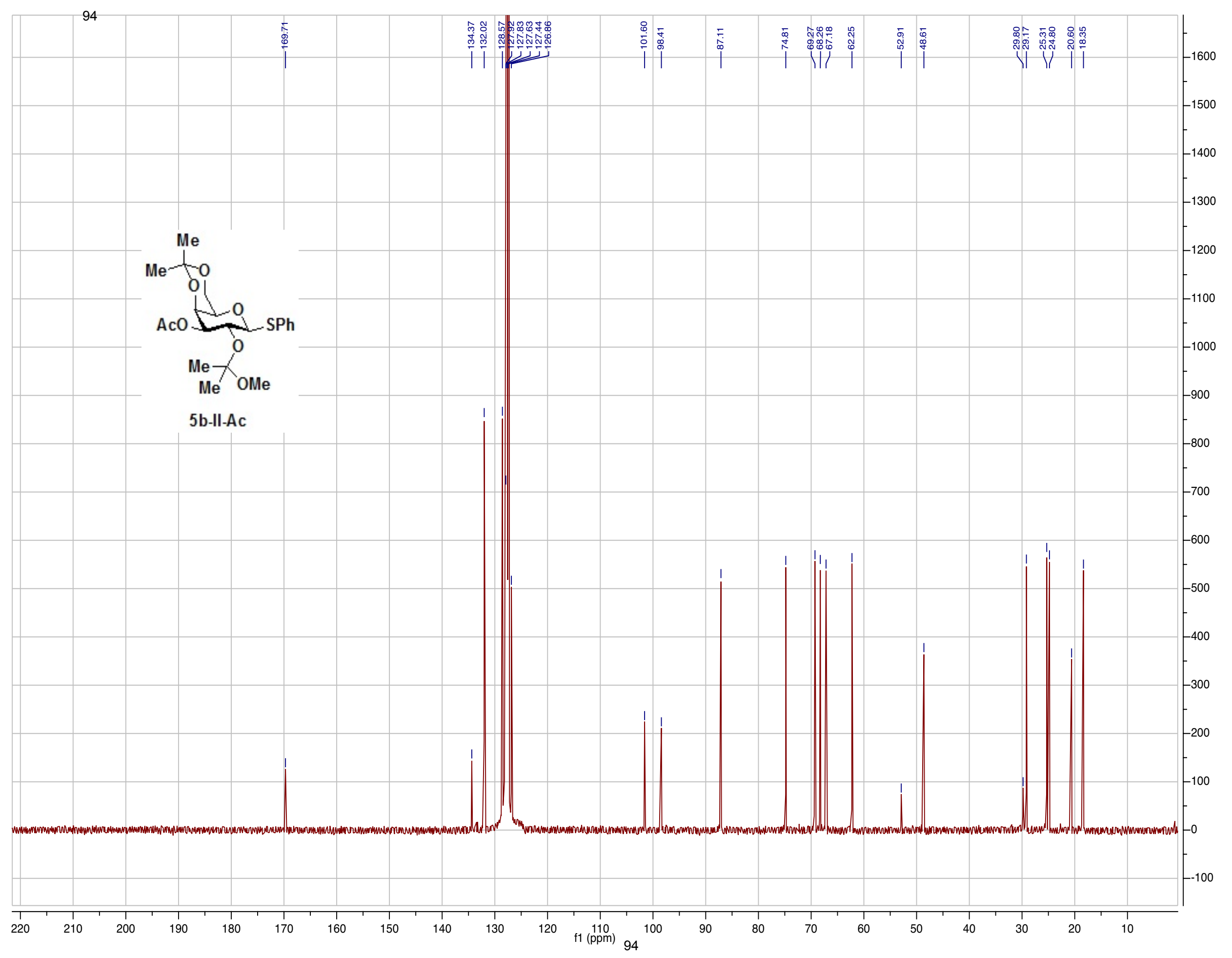




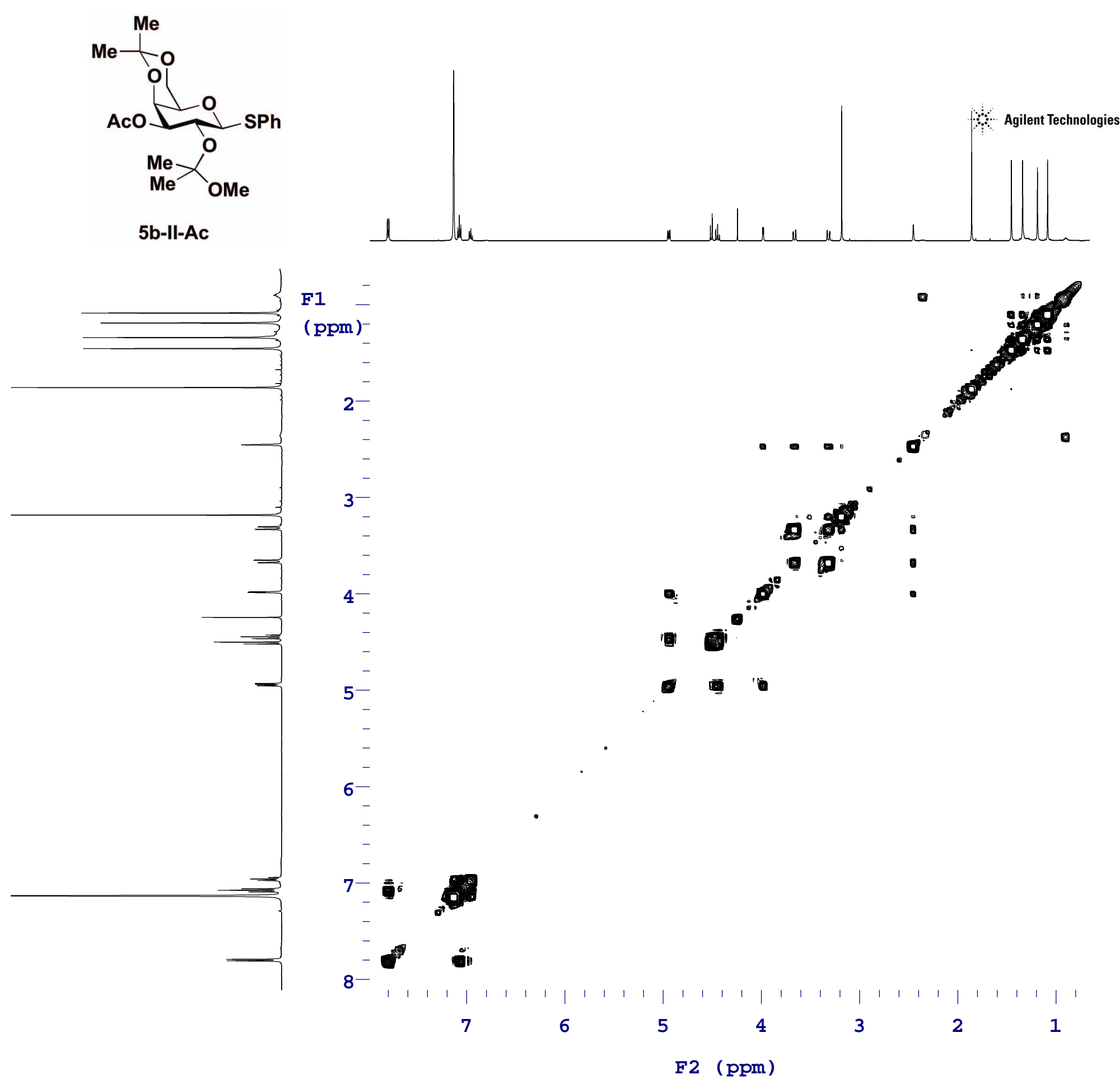




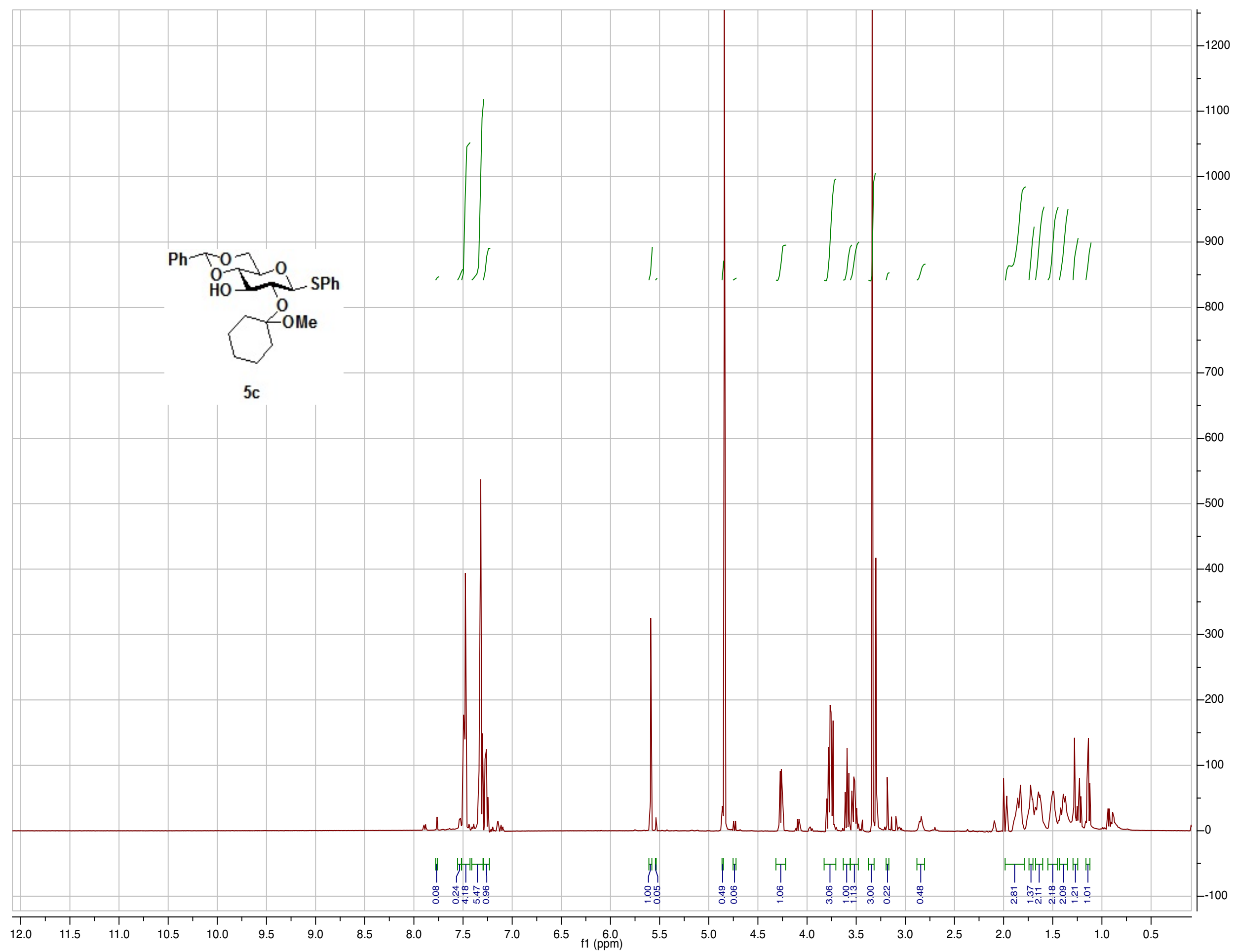




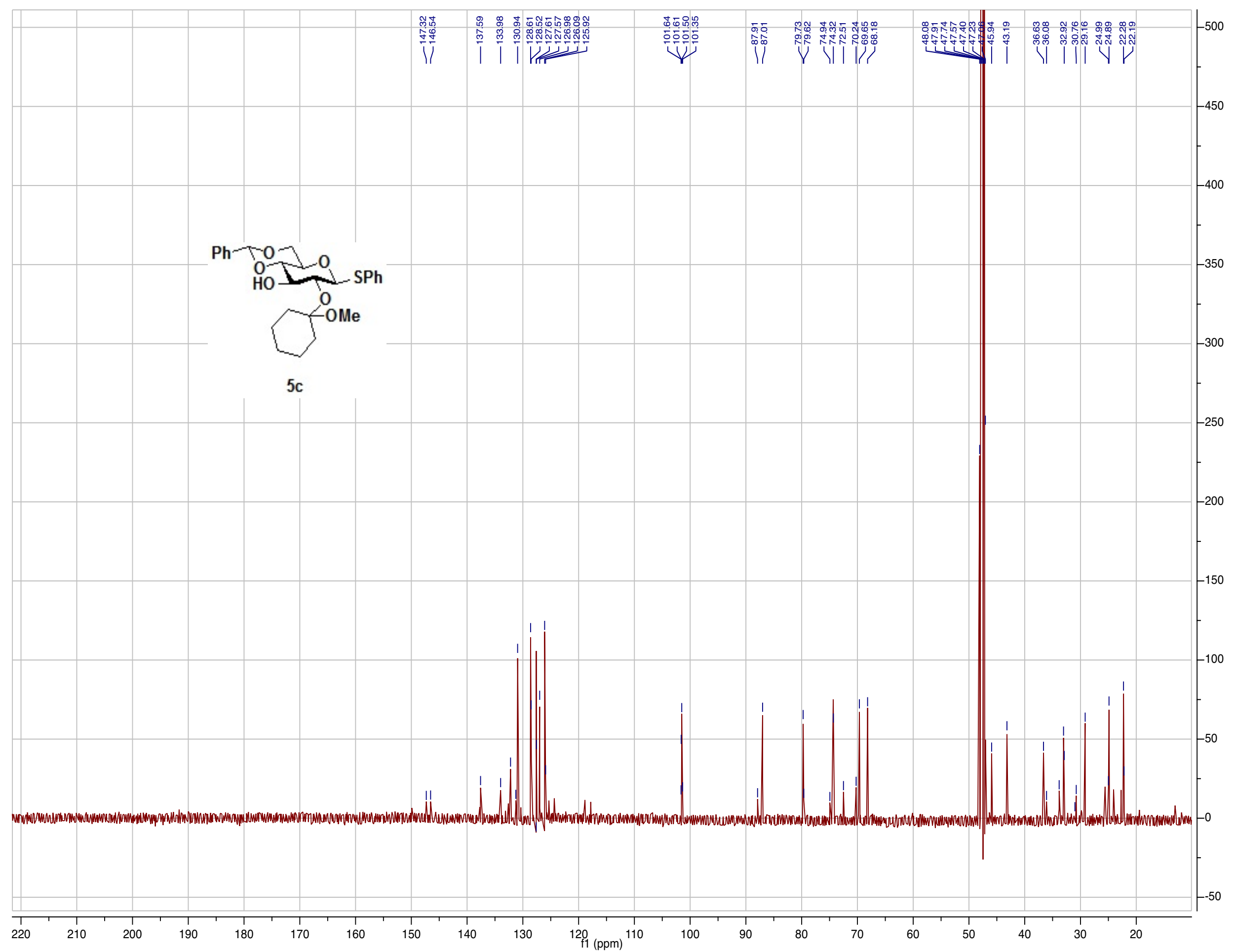




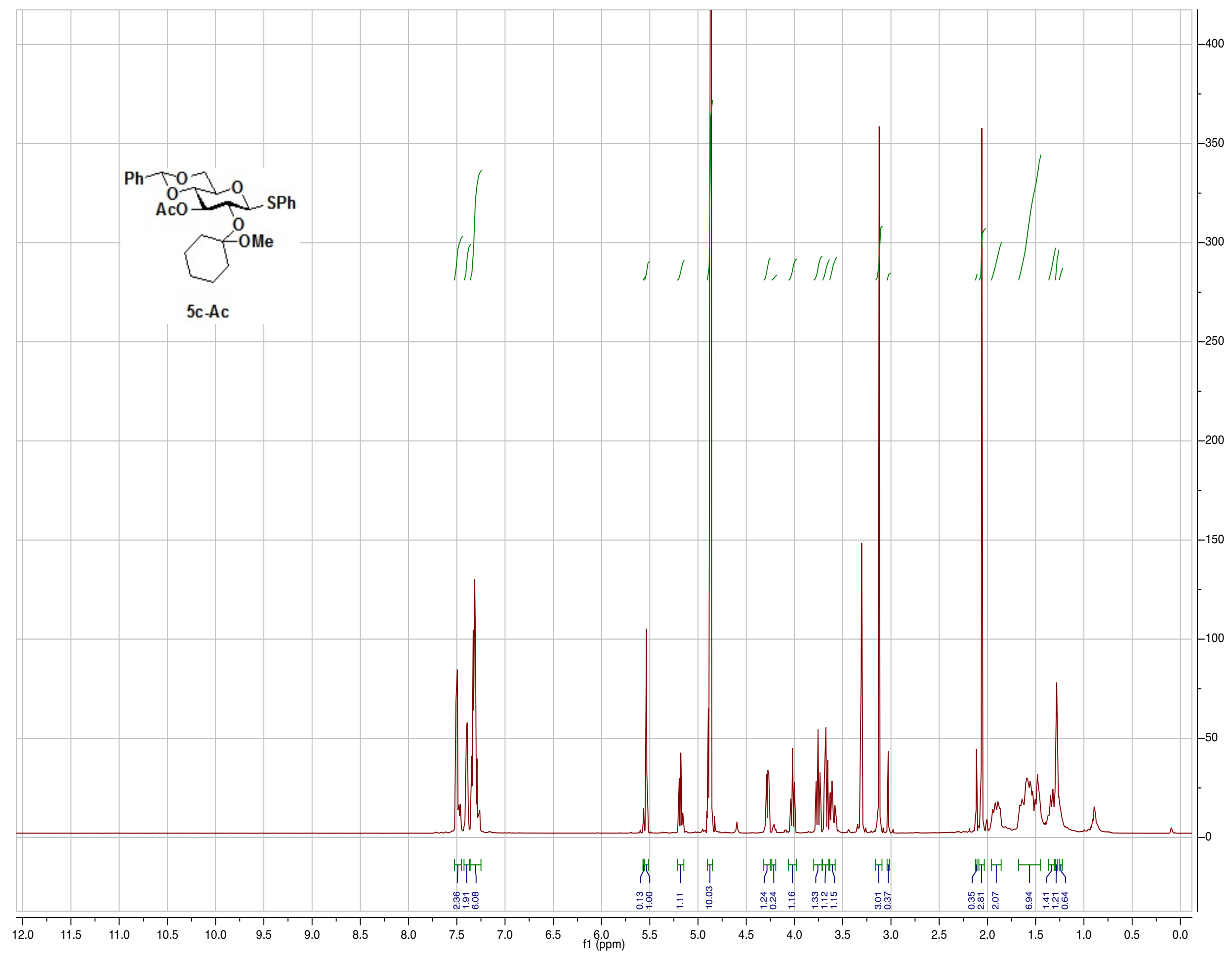




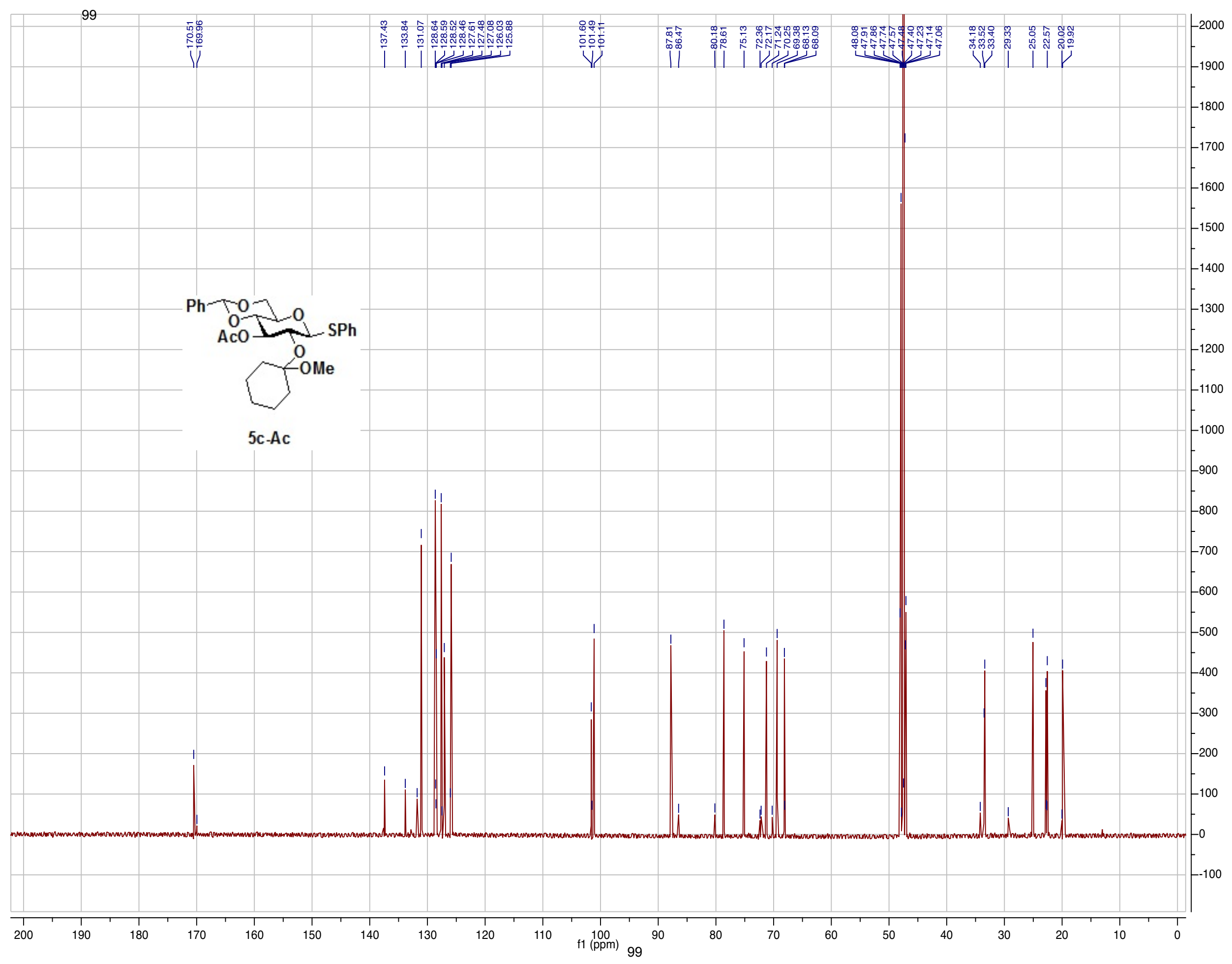


Sample Name:

EM-04-112PURECOSY-METHANOL-D4 Data Collected on:

Sn. Chem. LSA. UMich . edu-inova 500 5c-Ac

Archive directory:

Sample directory:

FidFile: EM-04-112PURECOSY-METHANOL-D4

Pulse Sequence: gCosy

Solvent: cd3od

Data collected on: Sep 142012

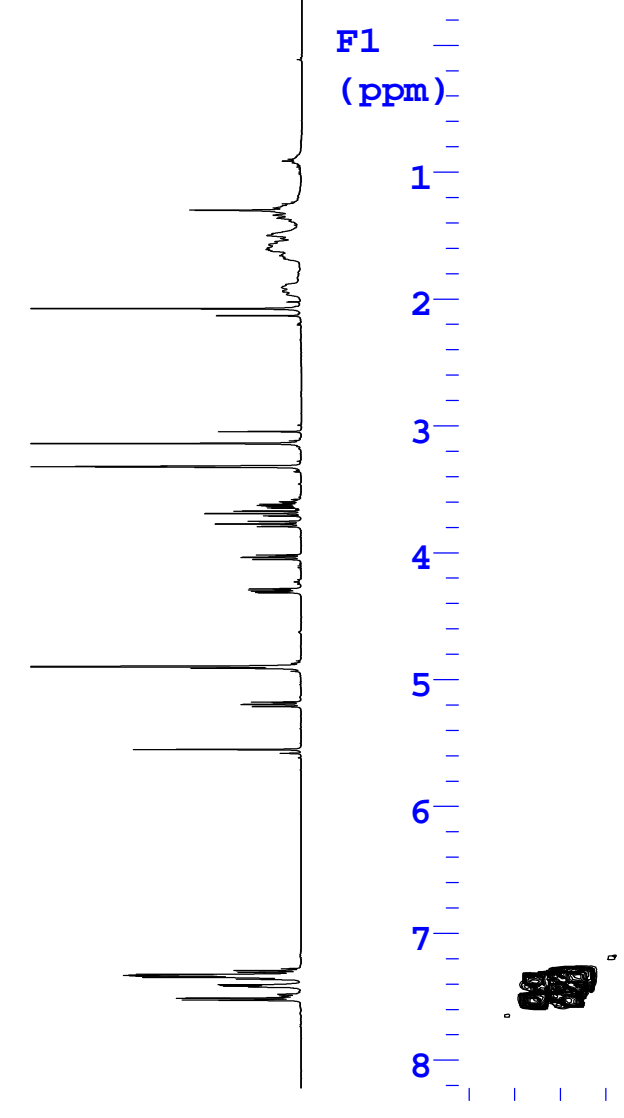

F1

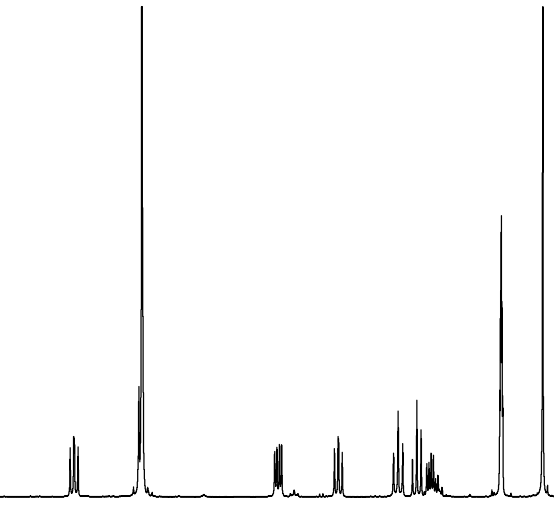

Agilent Technologies

7
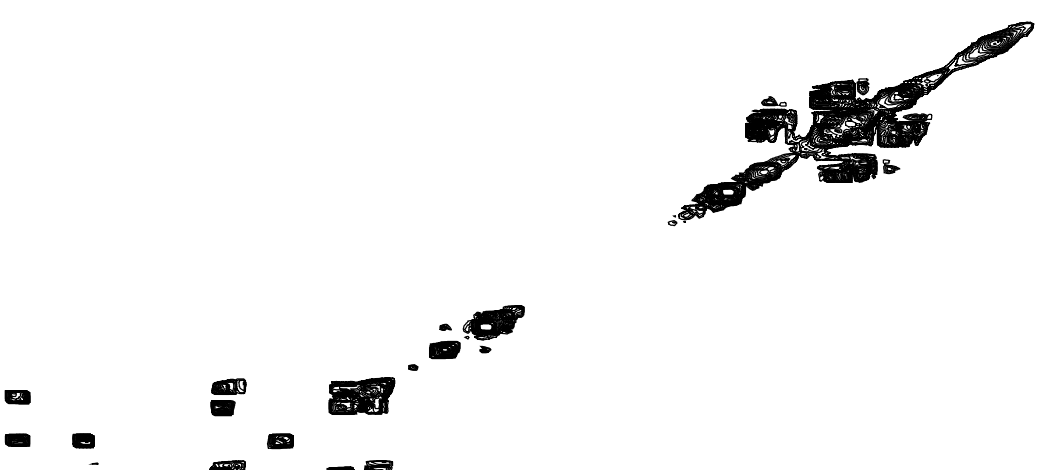

-

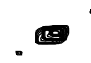




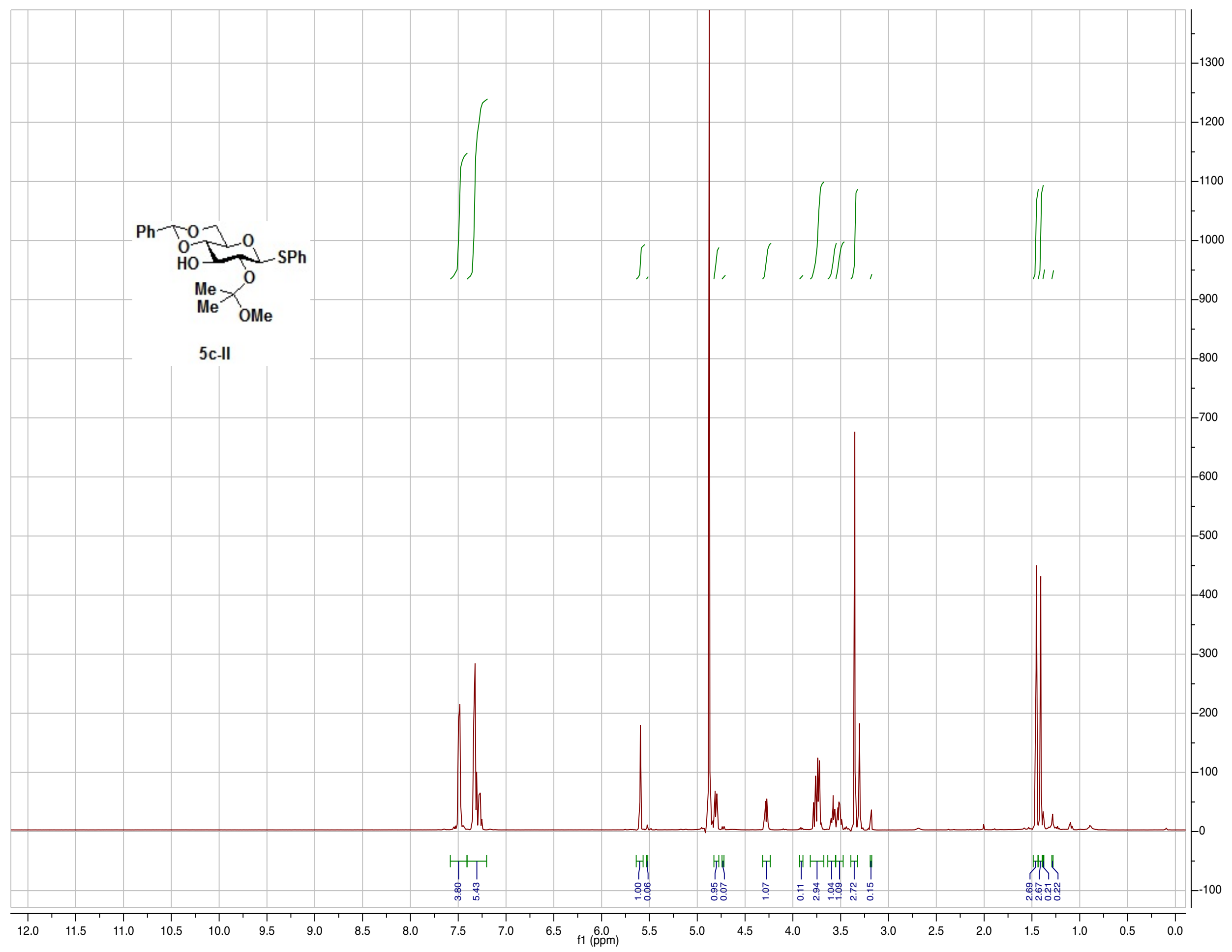




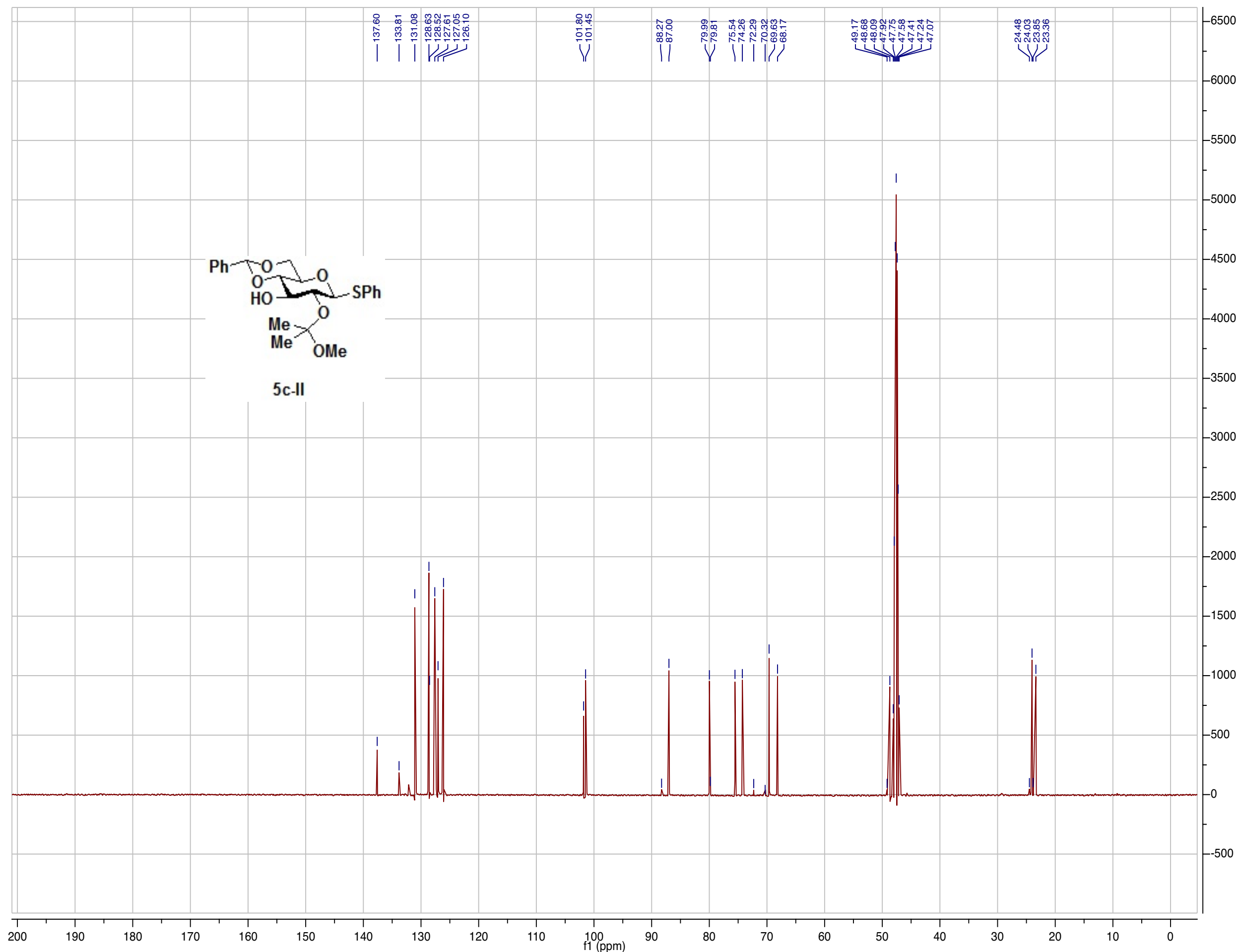




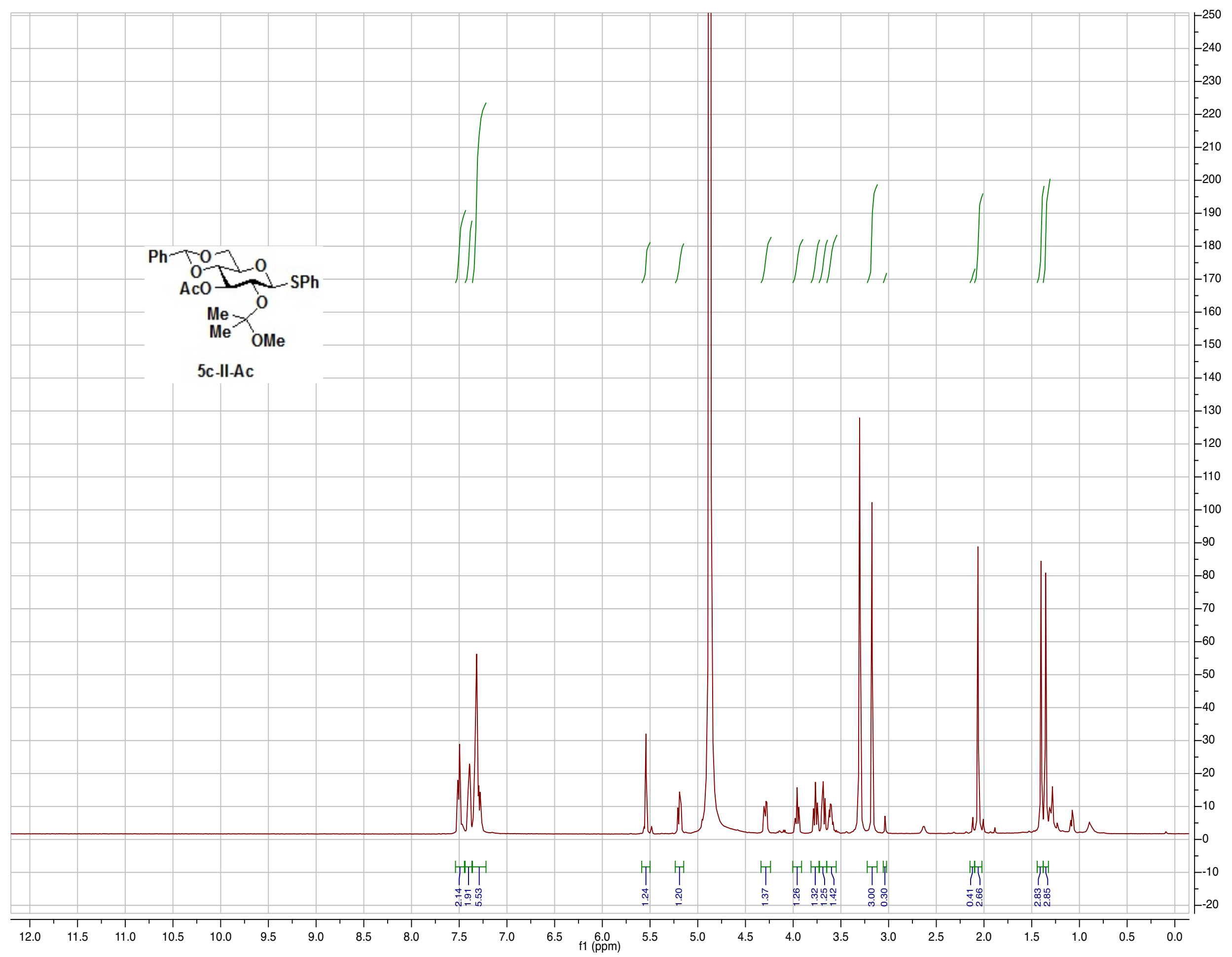




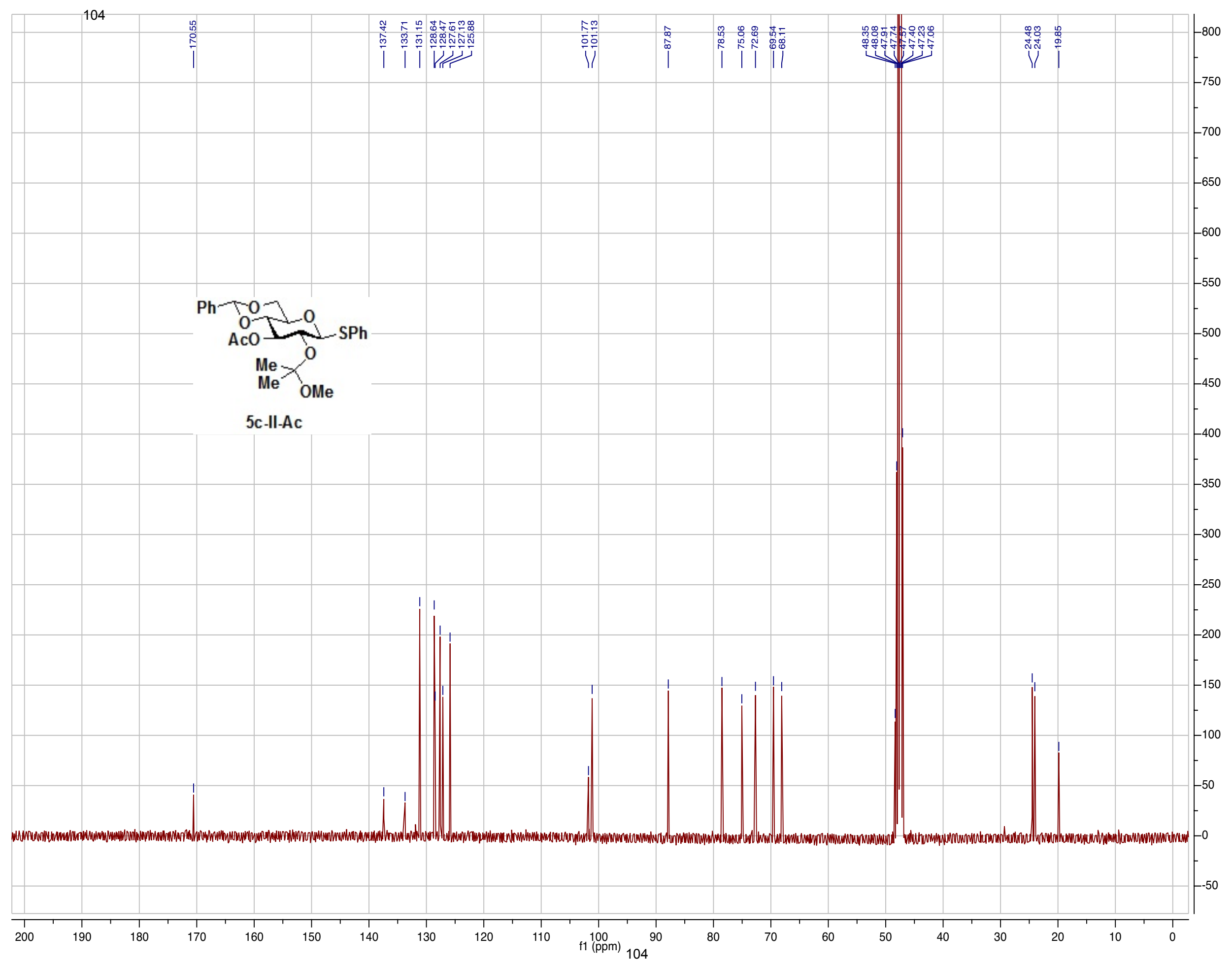


$\mathrm{Ph}$ Tro $\mathrm{O}$ SPh

Sample Name:

EM-04-132PURECOSY-METHANOL-D4 Data Collected on:

Sn. Chem. LSA . UMich . edu-inova500 Archive directory:

Sample directory:

FidFile: EM-04-132PURECOSY-METHANOL-D4

Pulse Sequence: gCOSY

Solvent: cd3od
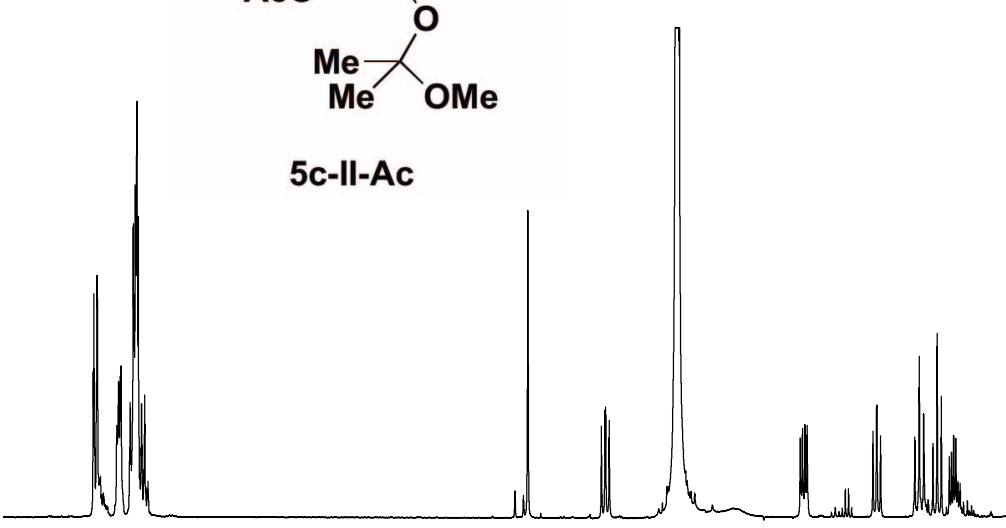

Data collected on: Sep 242012
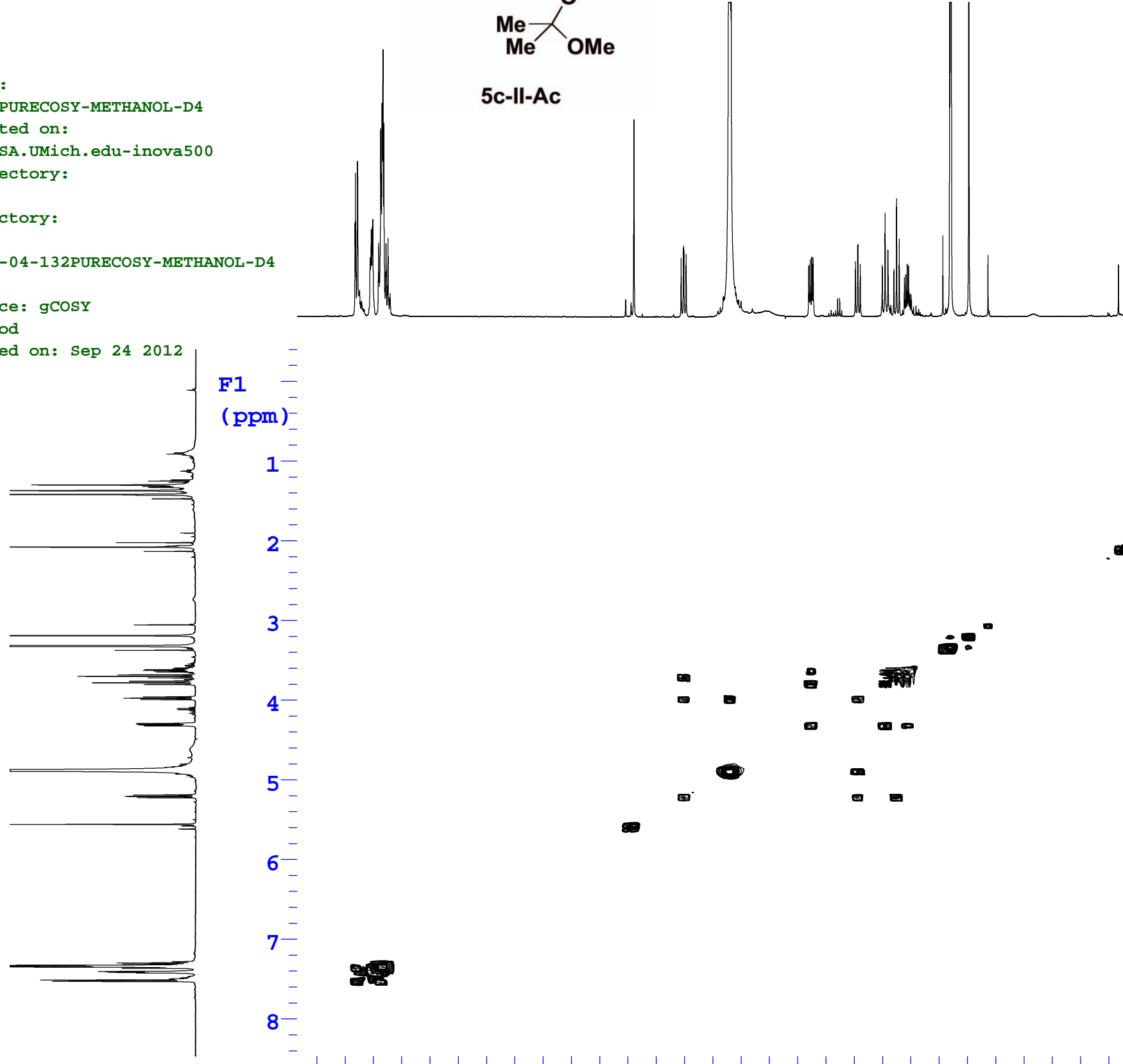


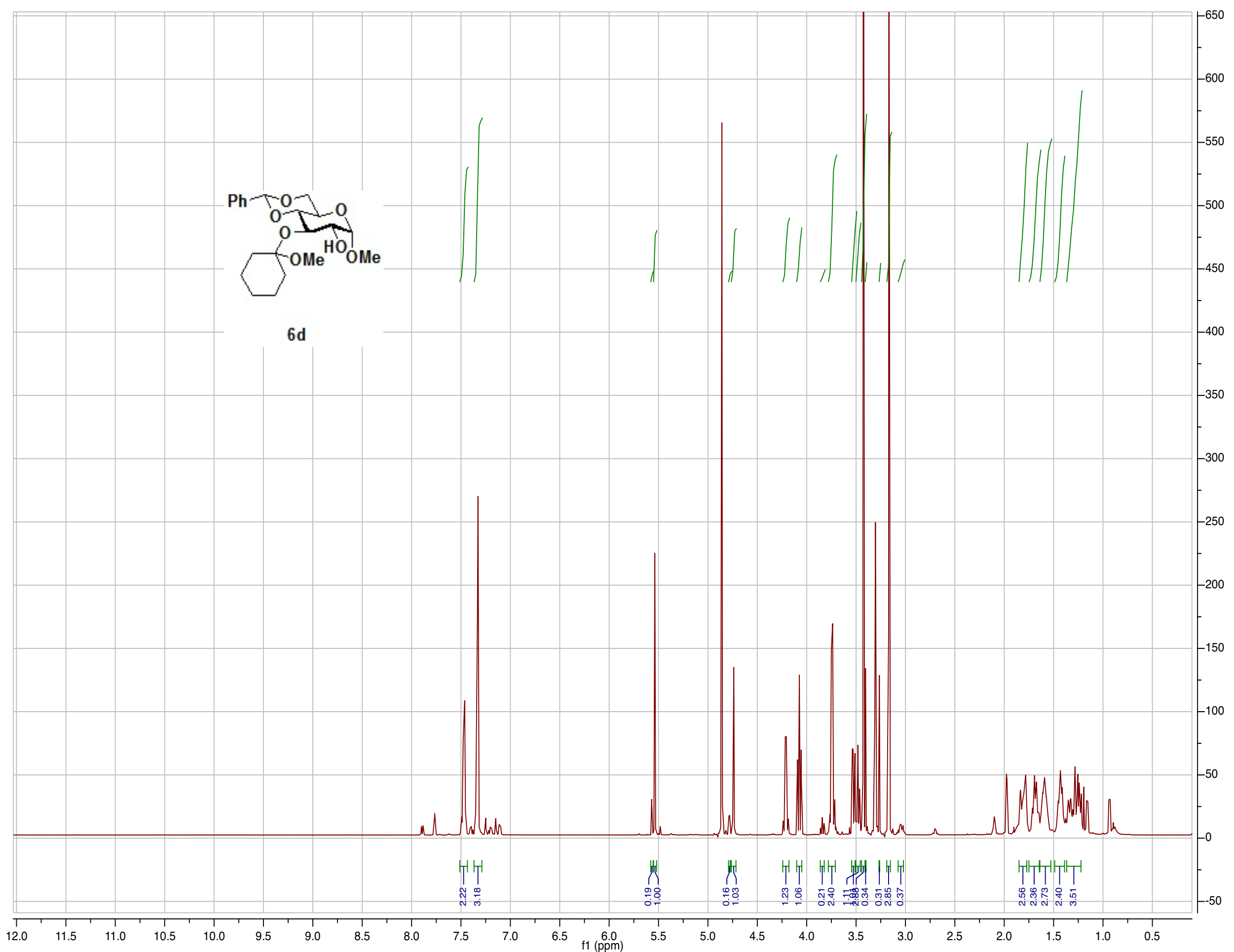




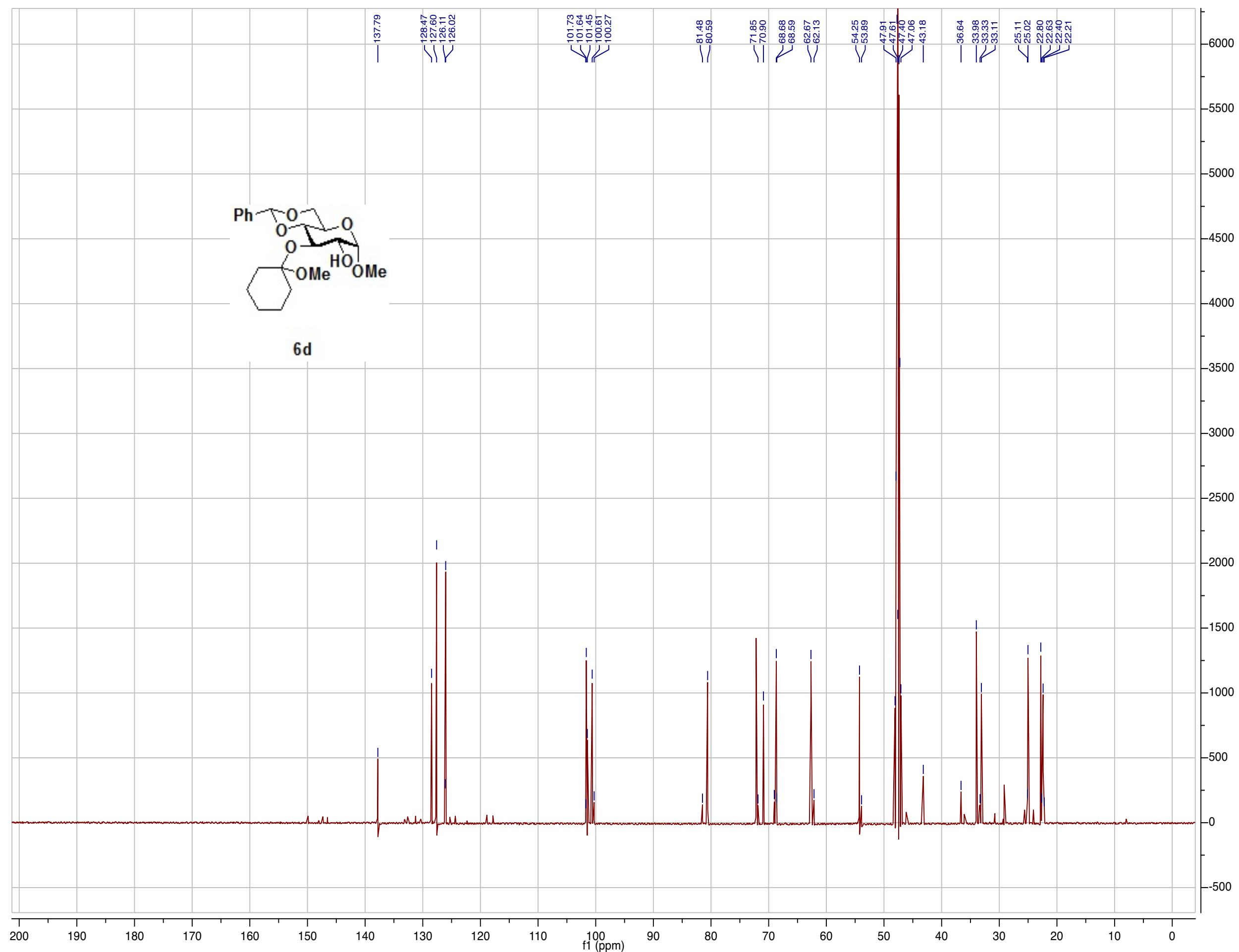




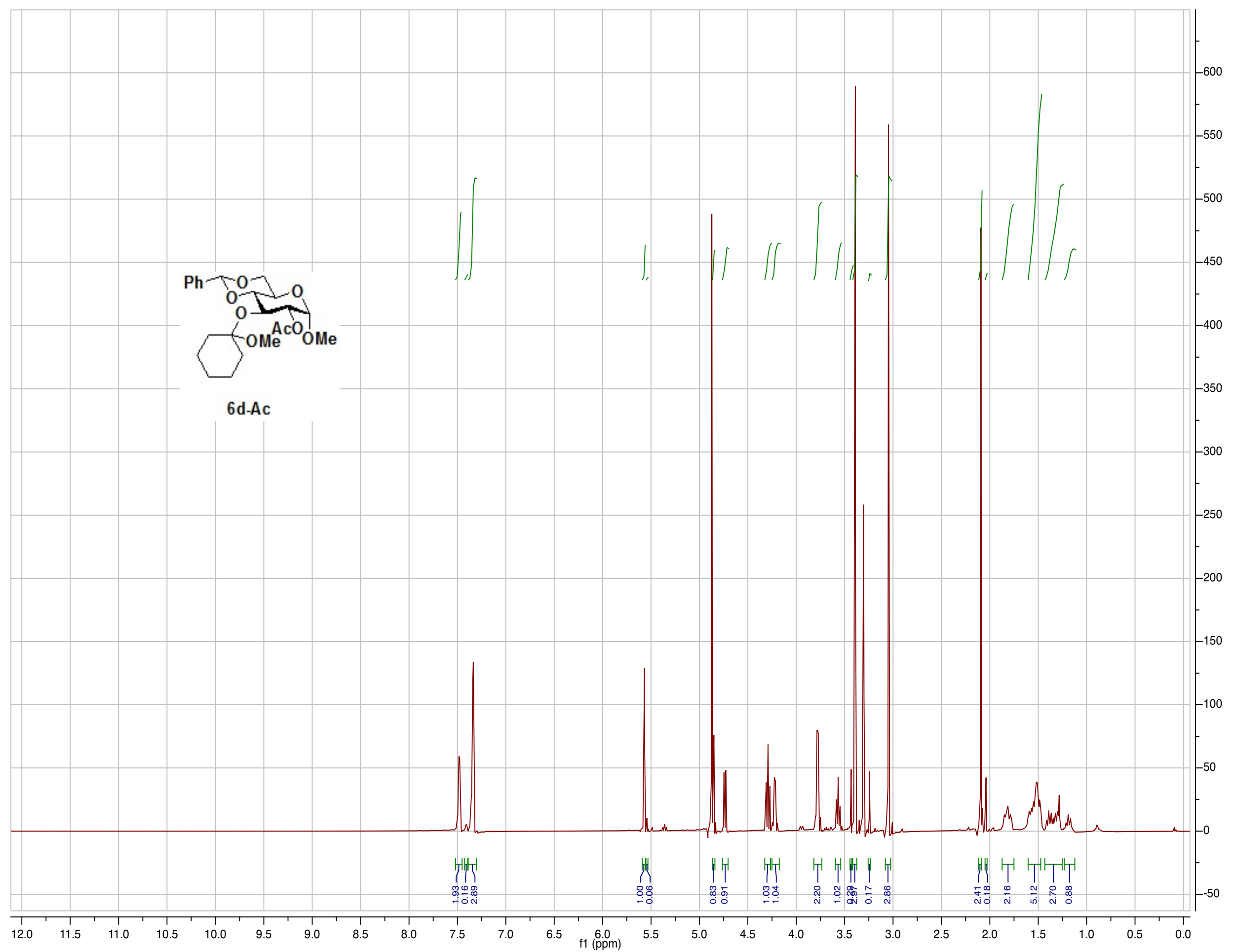




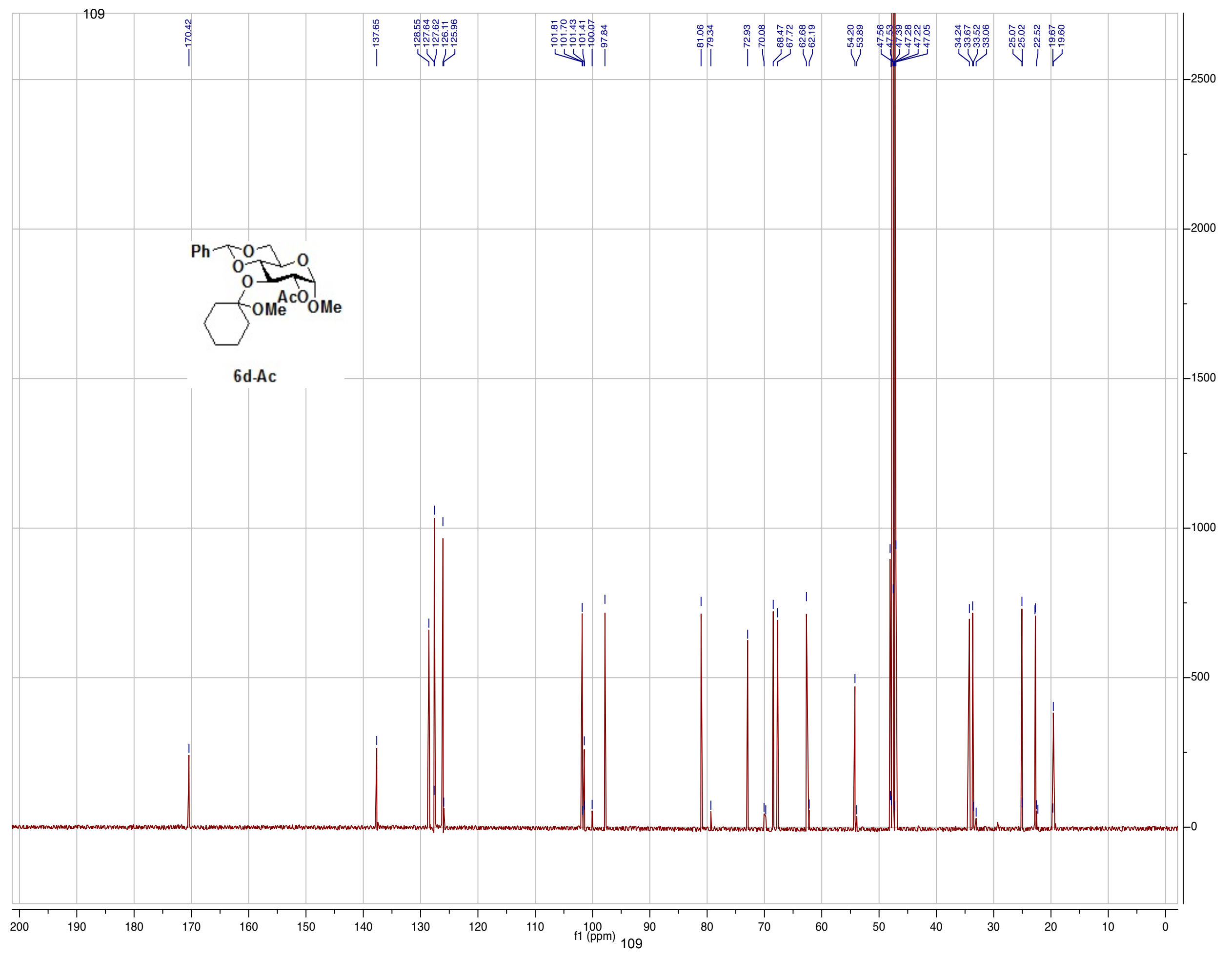


STANDARD PROTON PARAMETERS

Sample Name:

EM-04-172PURECOSY-METHANOL-D4 Data Collected on:

$$
\text { Te-vnmrs500 }
$$

Archive directory:

Sample directory:

FidFile: EM-04-172PURECOSY-METHANOL-D4

Pulse Sequence: gcosy

Solvent: cd3od

$\mathrm{Ph}$

Data collected on: Oct 122012
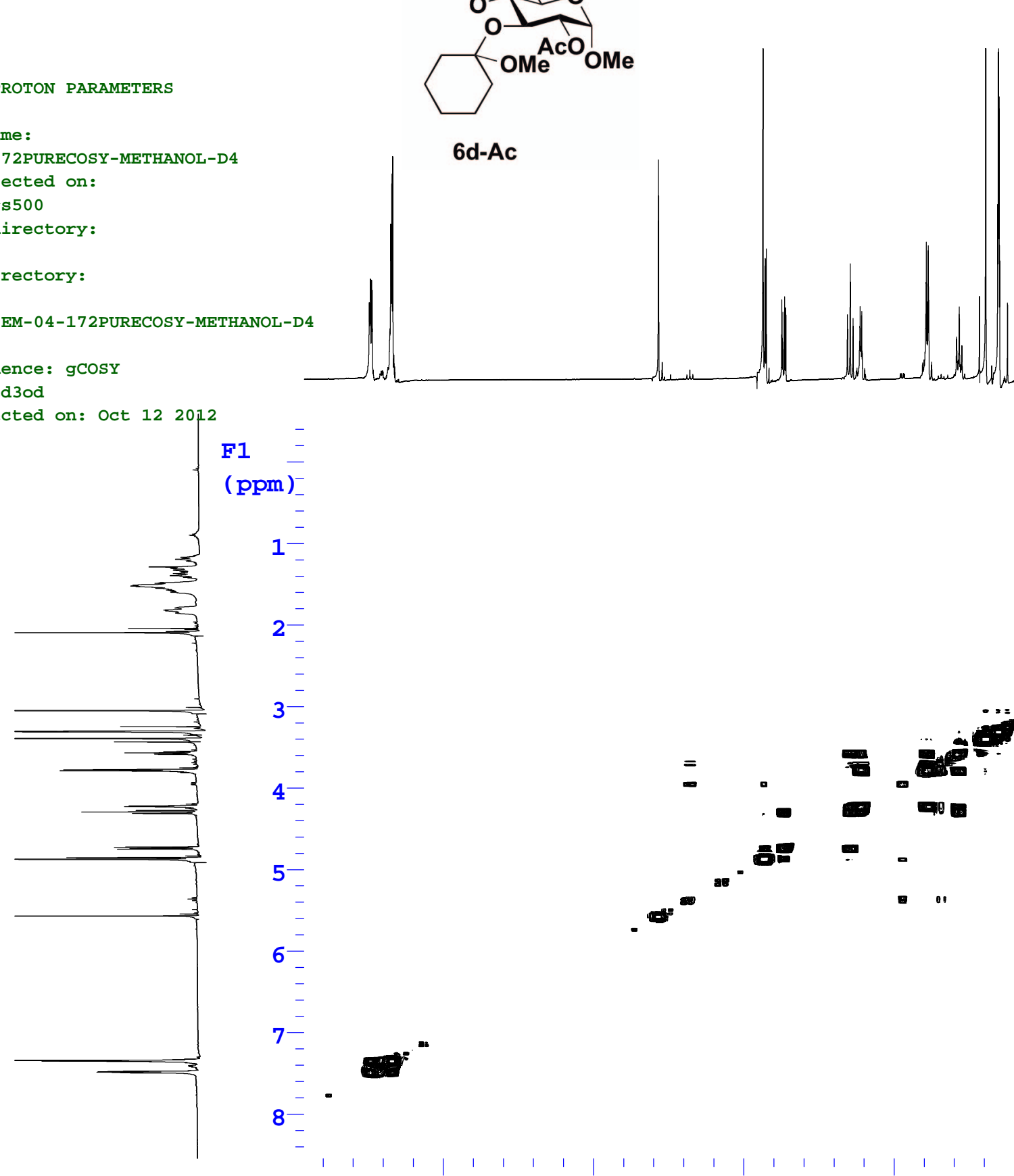

Agilent Technologies

7

6

5

4

3

2

1 


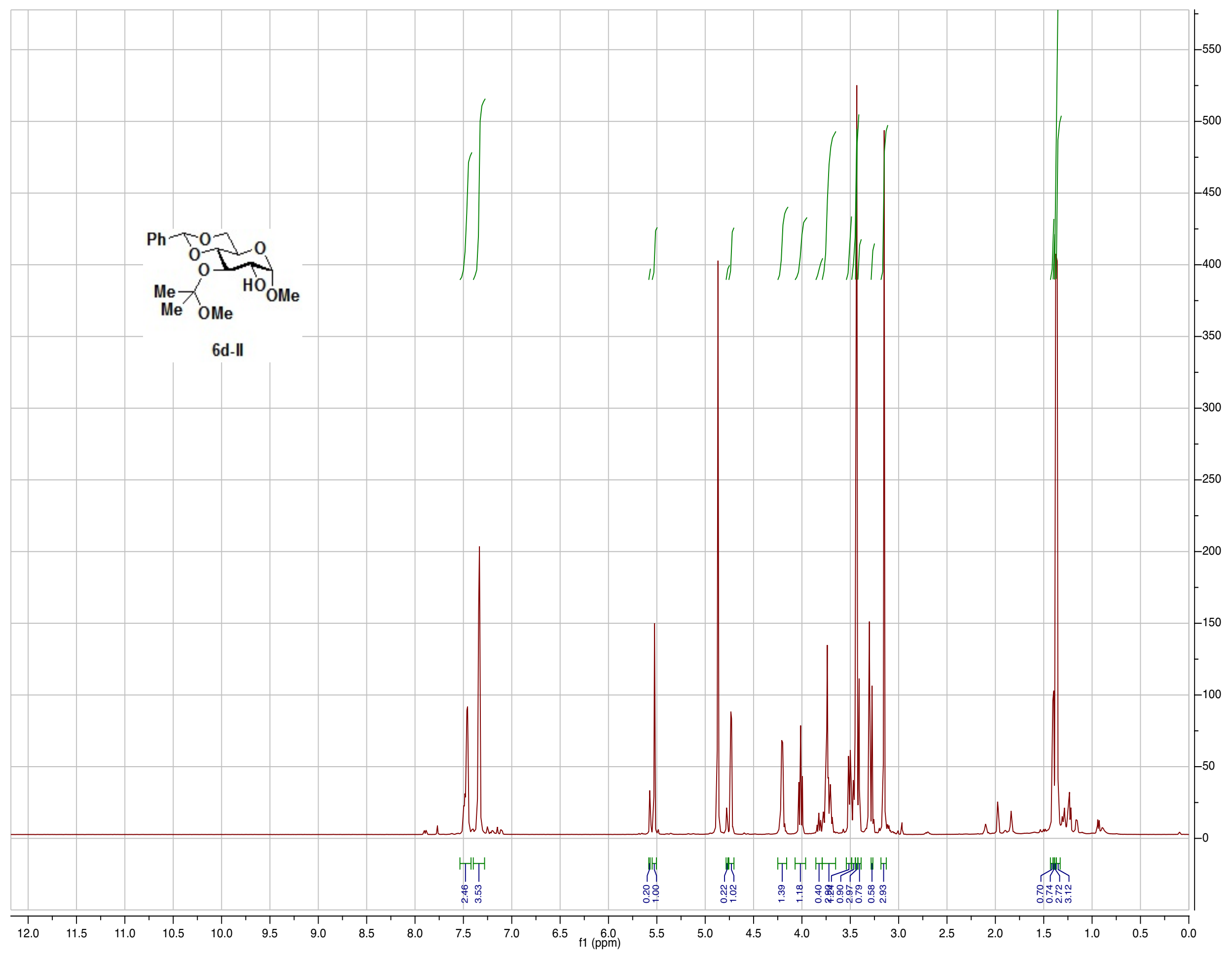




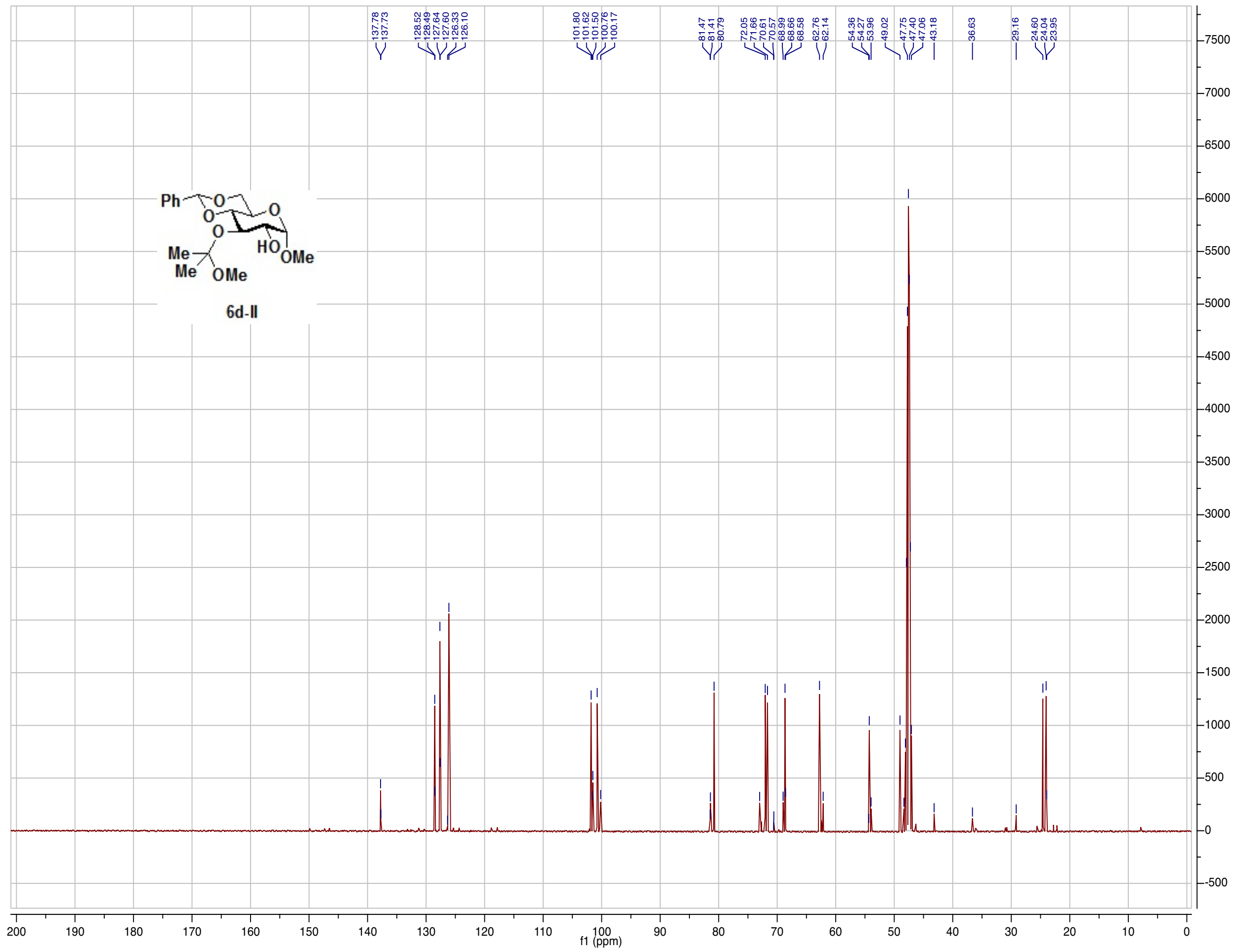




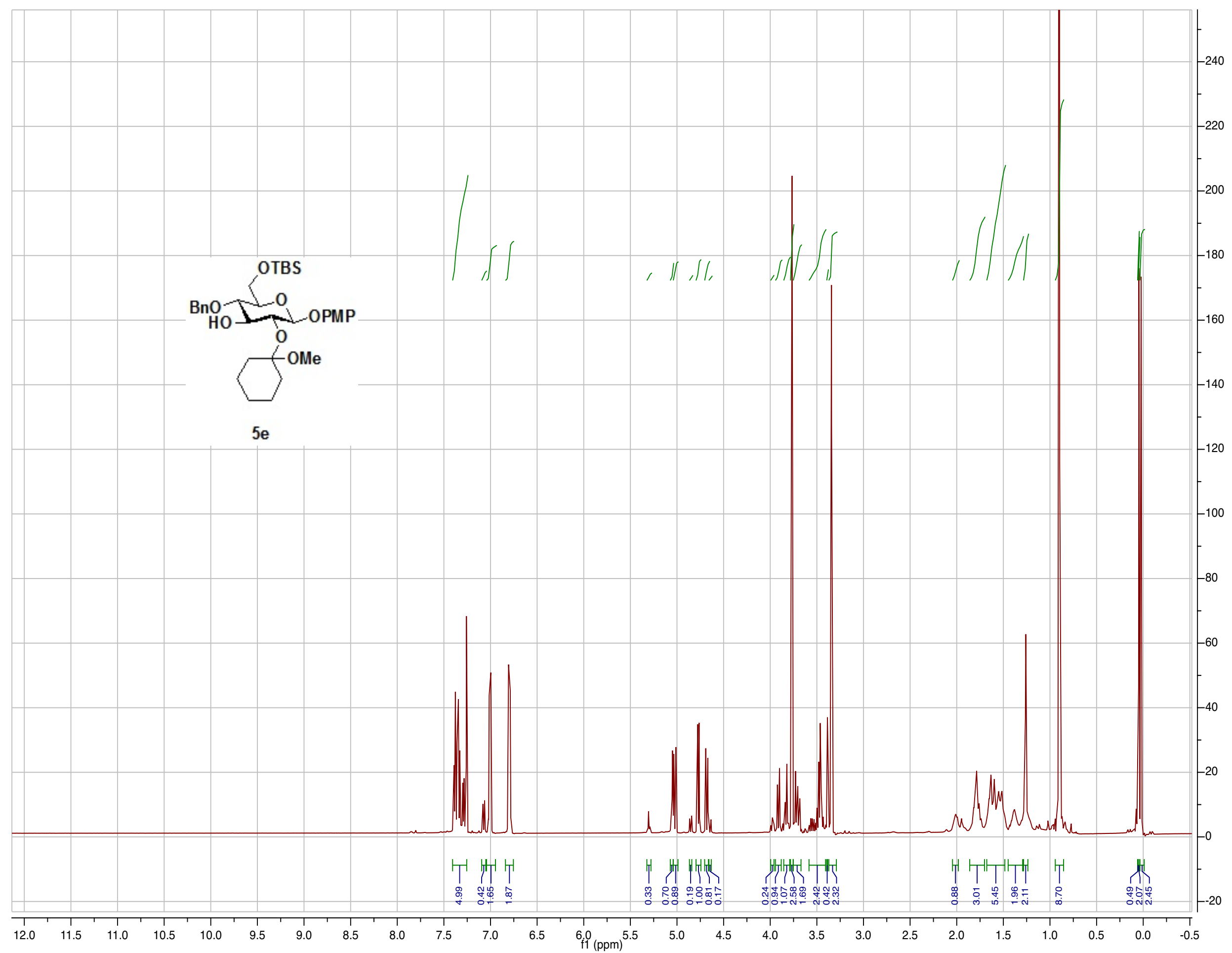




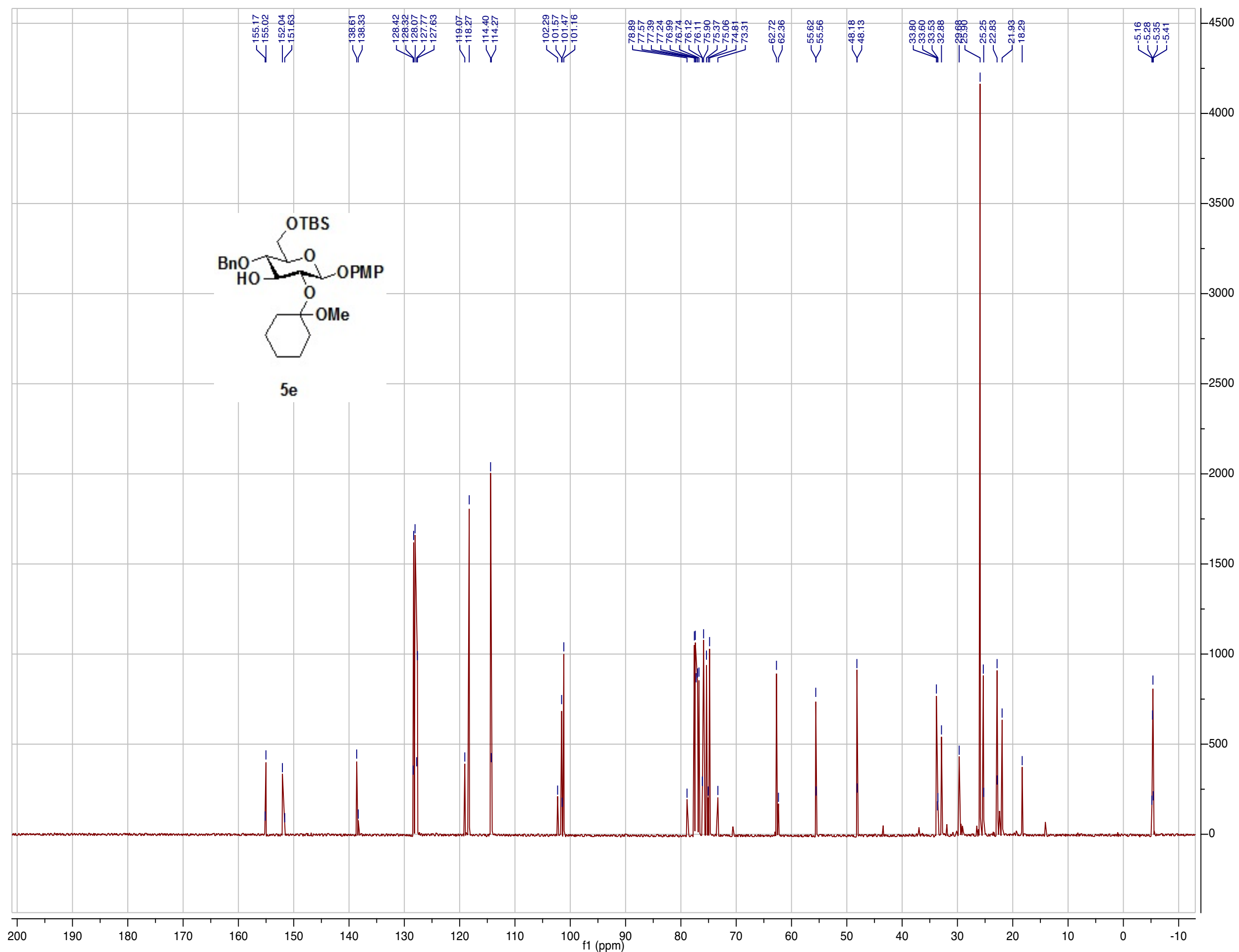




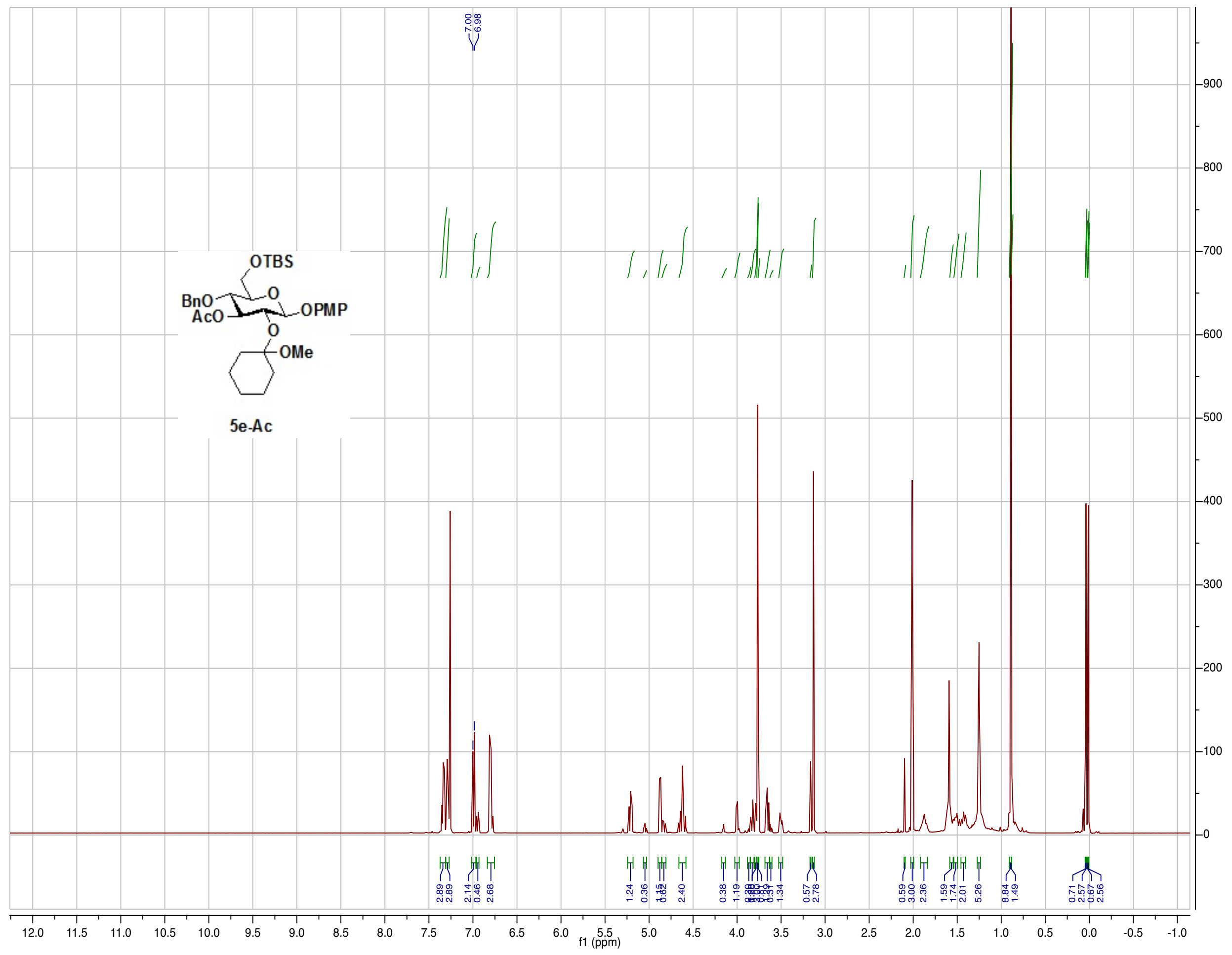




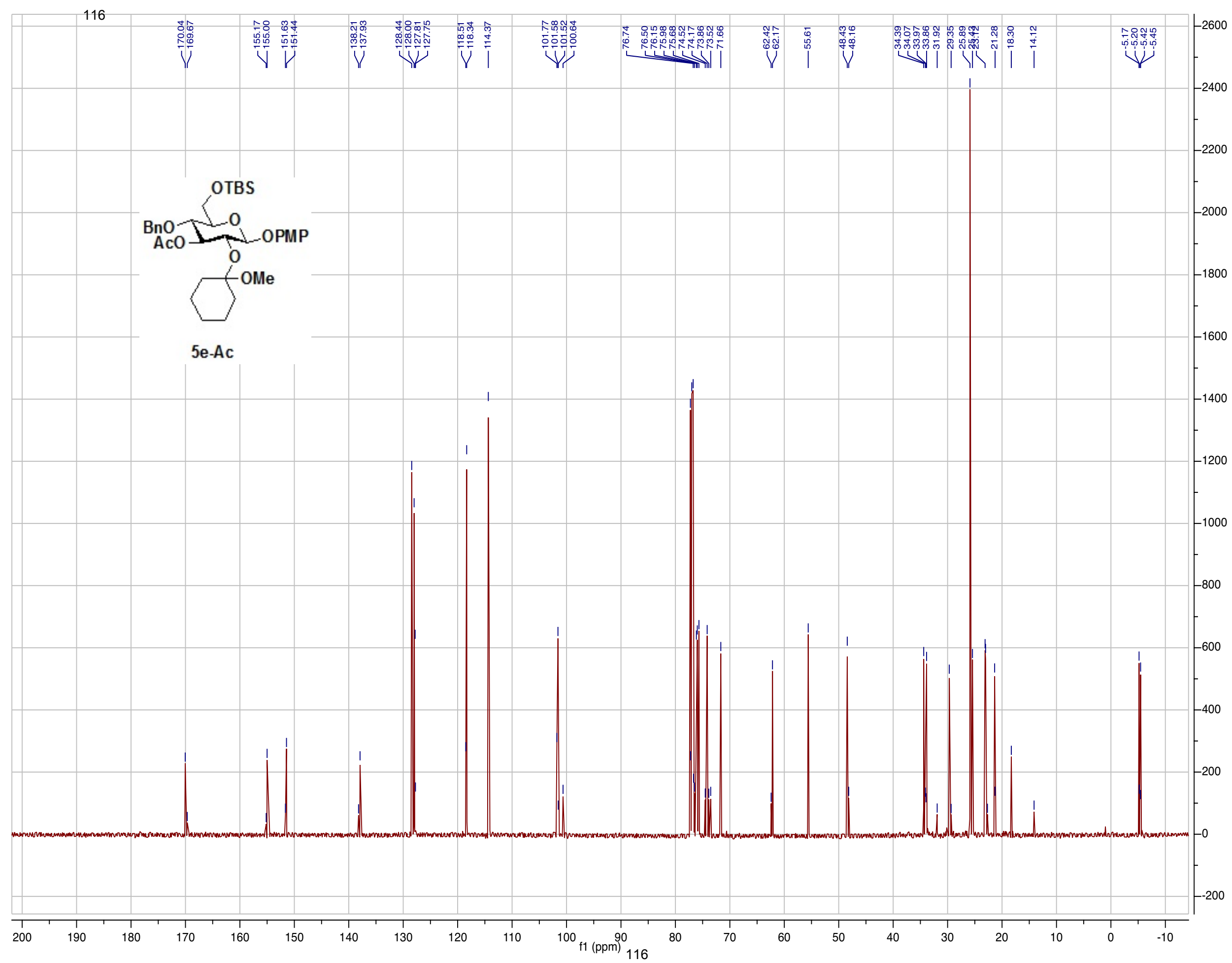


Sample Name:

EM-04-184PURECOSY-CDCL3

Data Collected on:

Sn. Chem. LSA. UMich. edu-inova 500

Archive directory:

Sample directory:

FidFile: EM-04-184PURECOSY-CDCL3

Pulse Sequence: gCOSY

Solvent: cdcl3

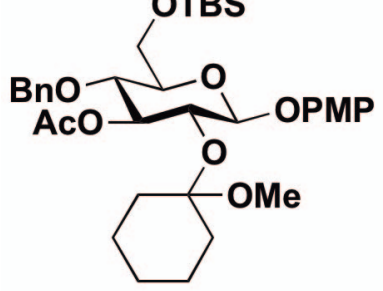

Data collected on: Oct 232012

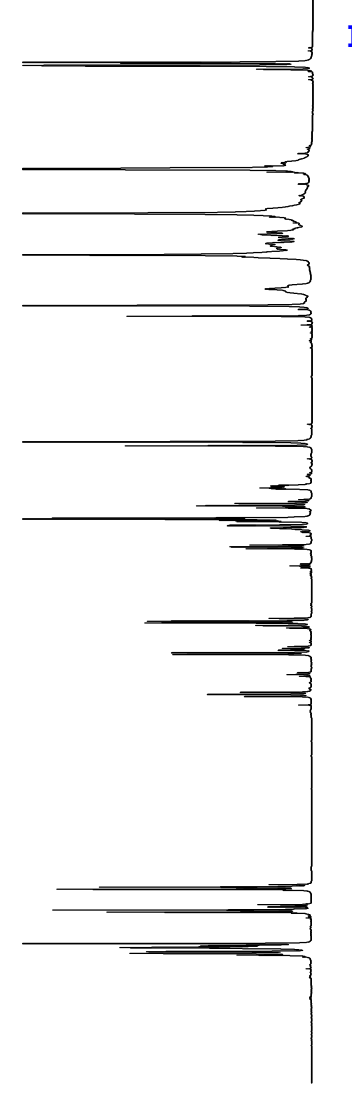

F1

(ppm)-

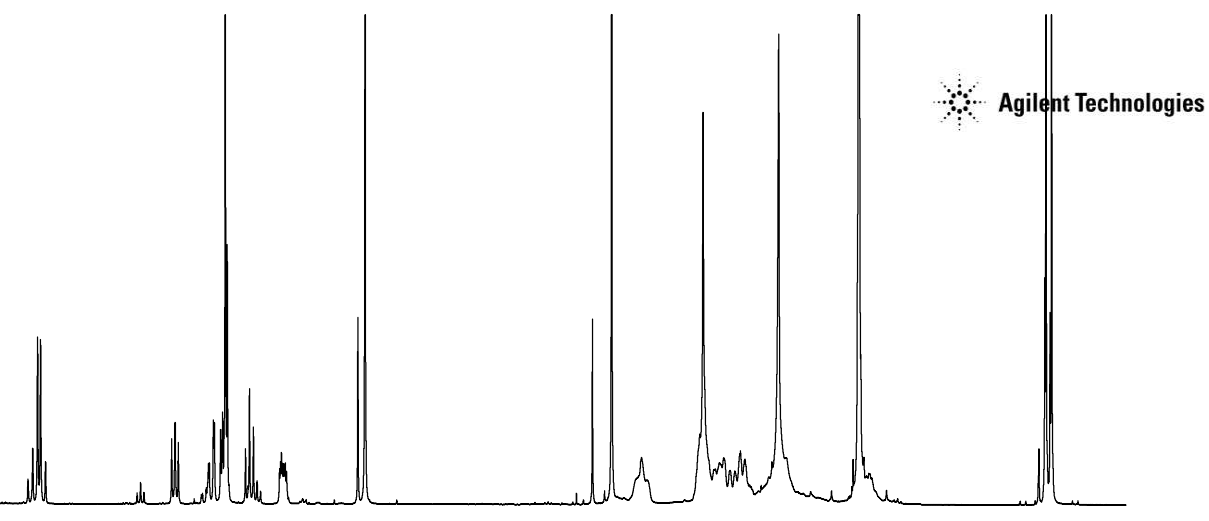

5e-Ac
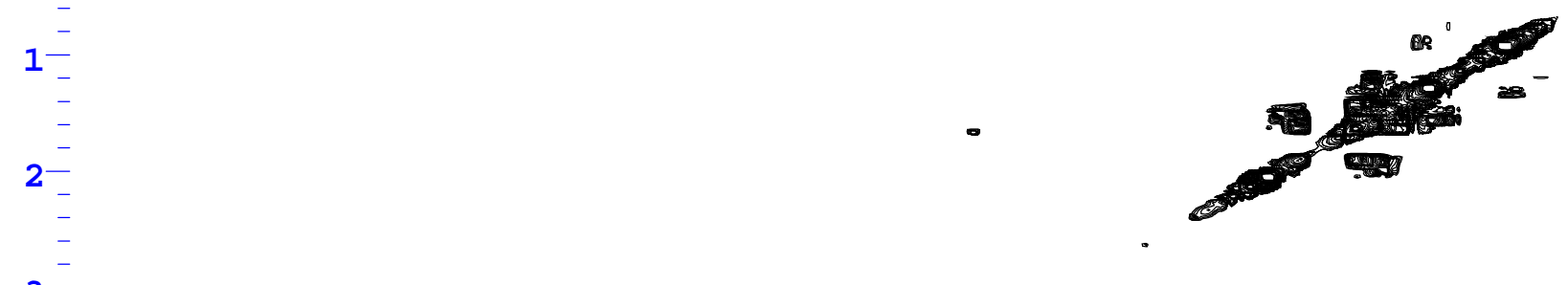

3-

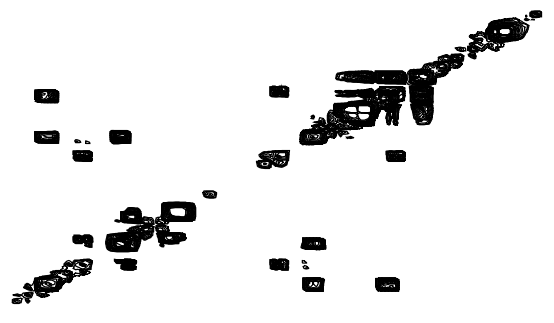

6-

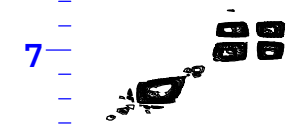

8- 


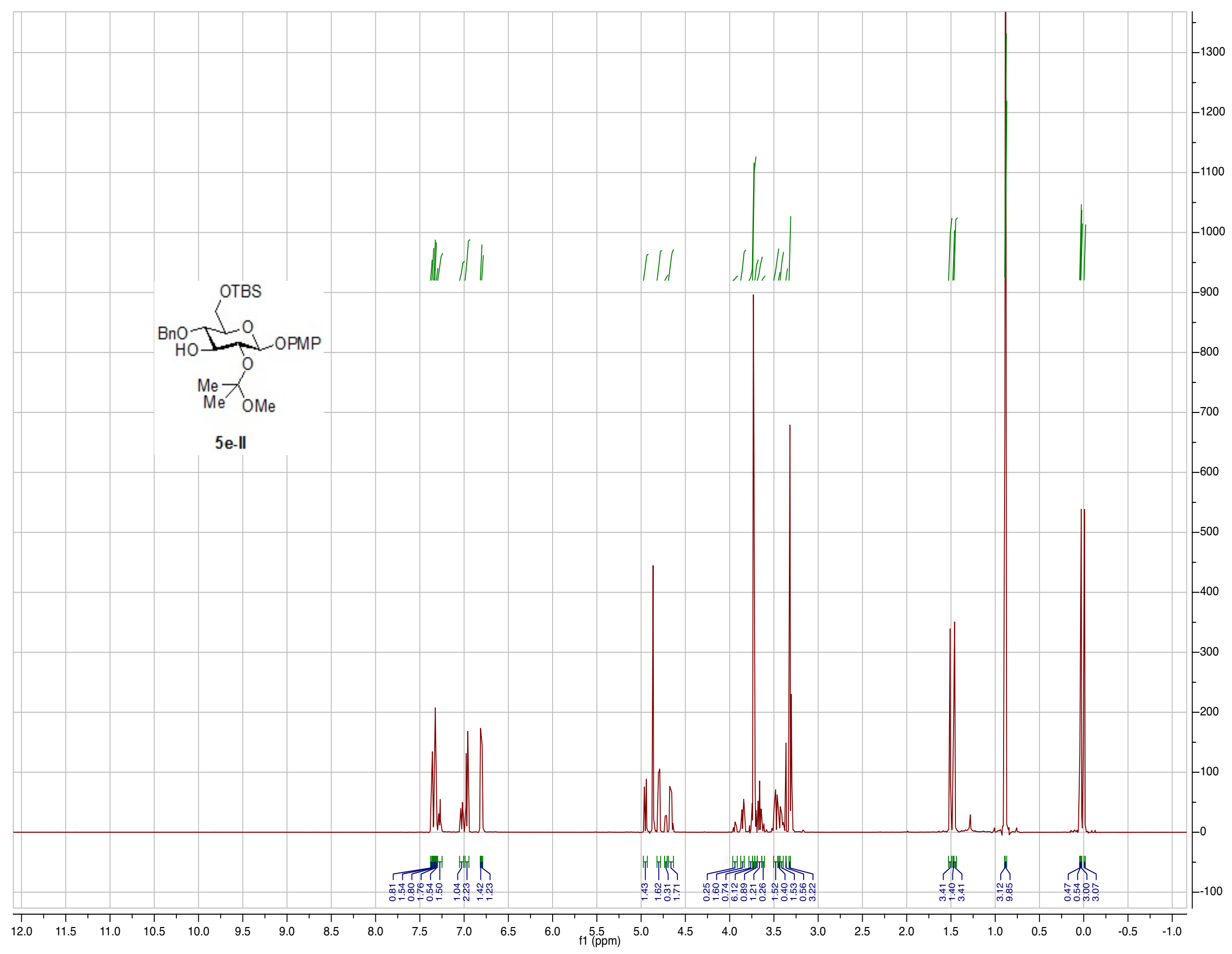




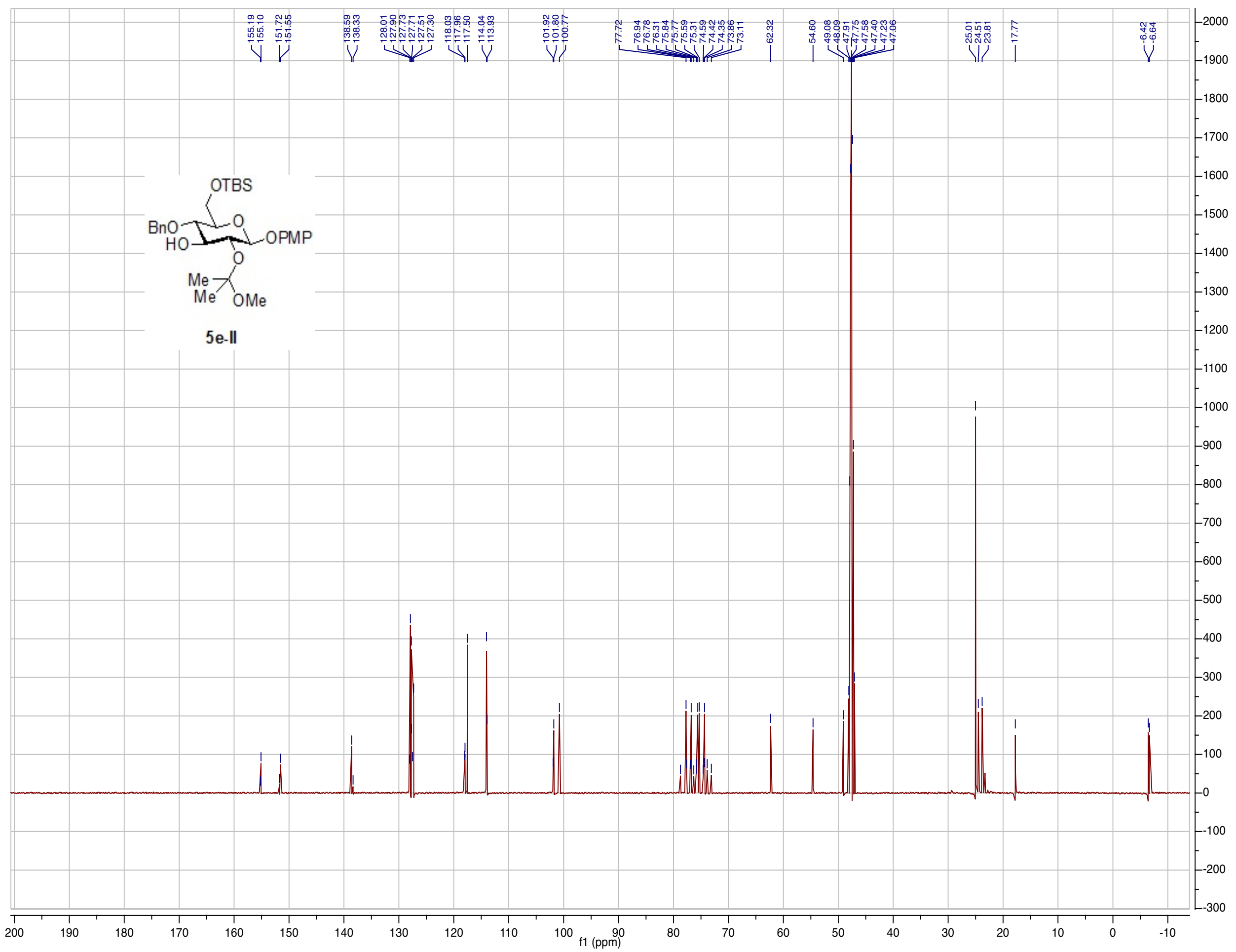




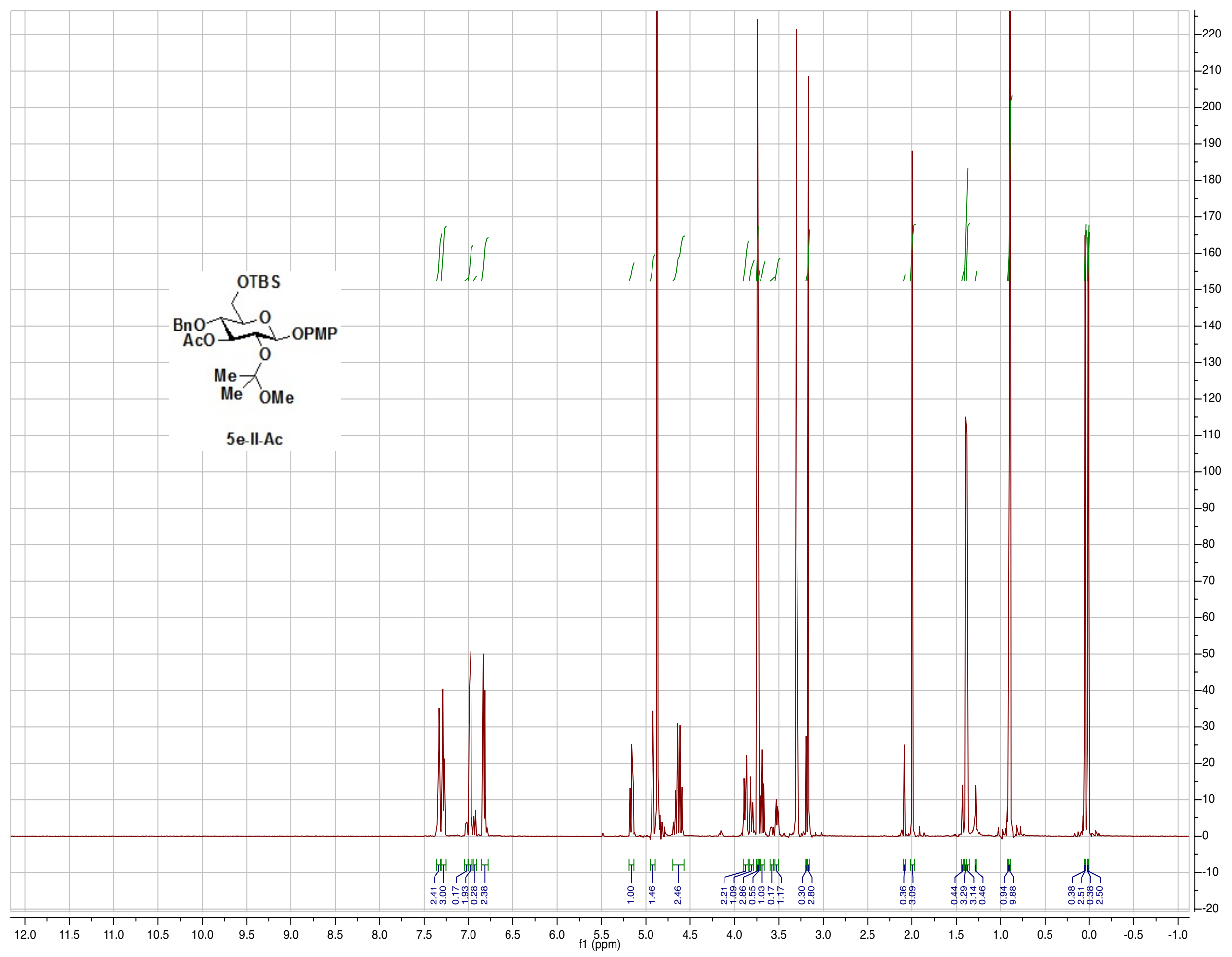




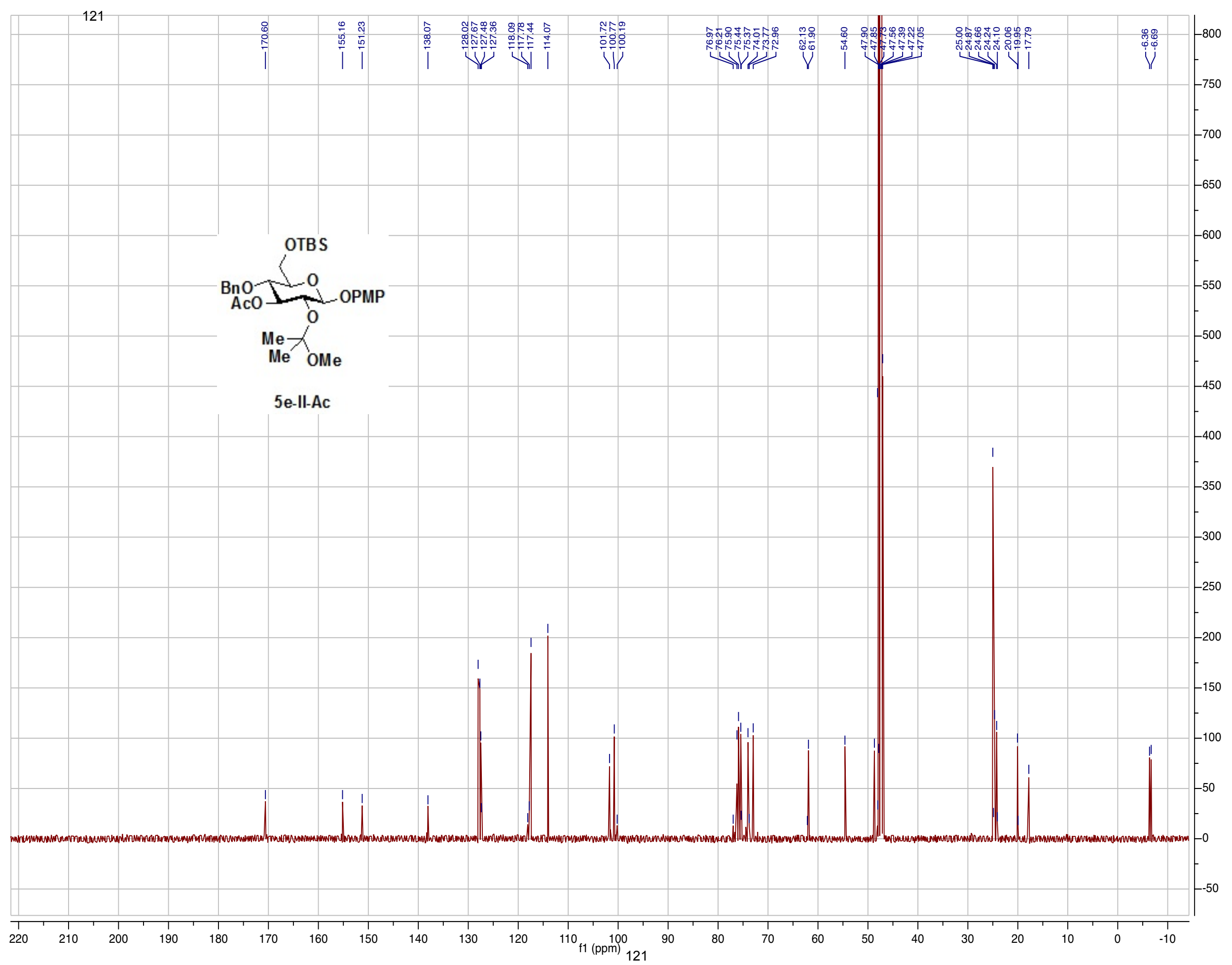


Automated Probe tuning parameter

Sample Name:

EM-05-78PURECOSY-METHANOL-D4 Data Collected on:

Te-vnmrs500

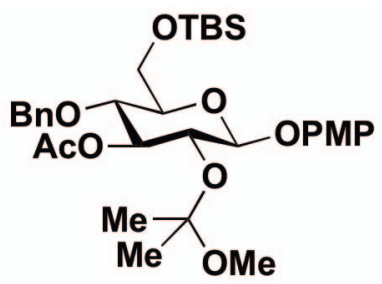

Archive directory:

\section{5e-II-Ac}

Sample directory

FidFile: EM-05-78PURECOSY-METHANOL-D4

Pulse Sequence: gcosy

Solvent: cd3od

Data collected on: Jan 182013
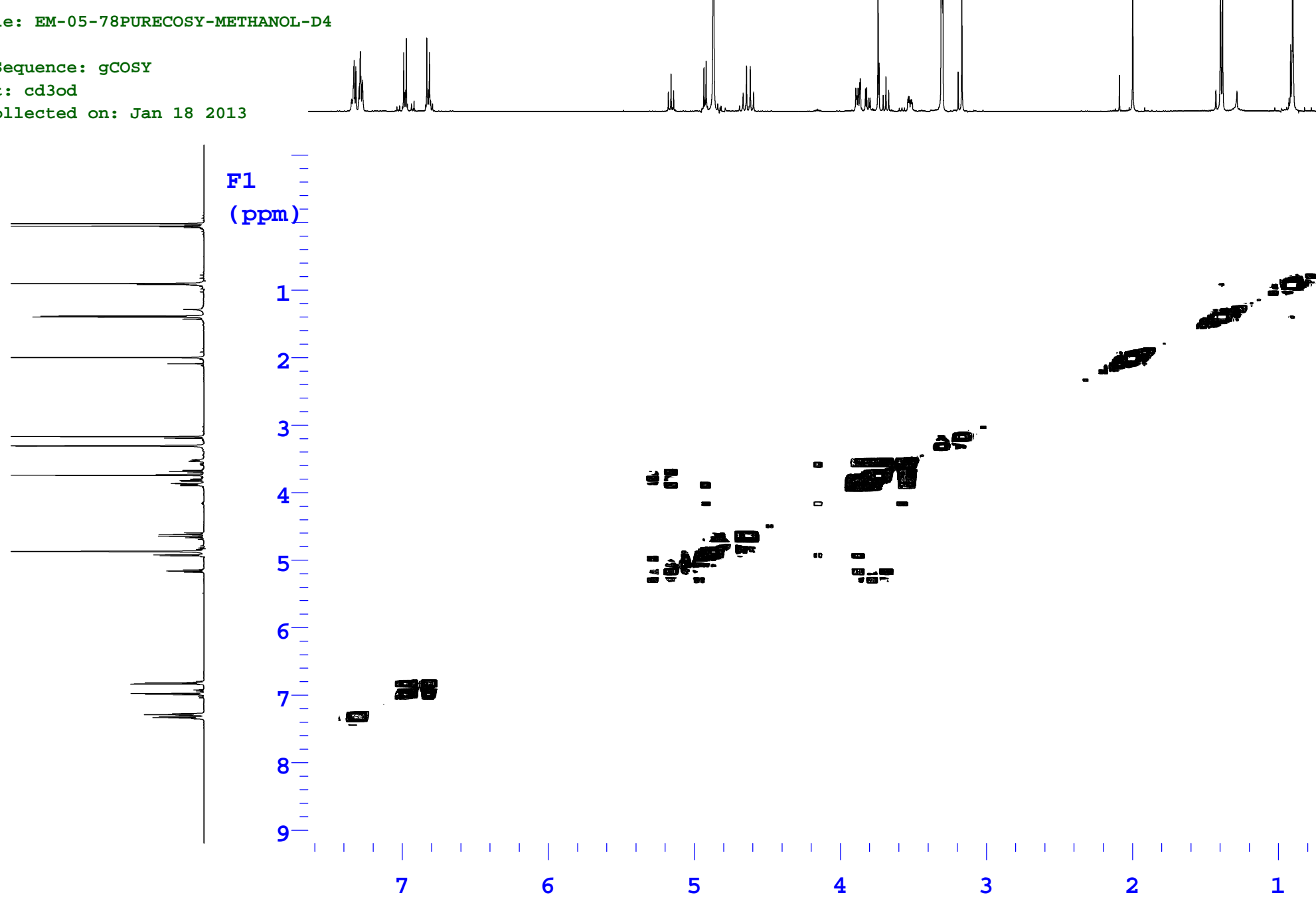

Agilent Technologies

F1 


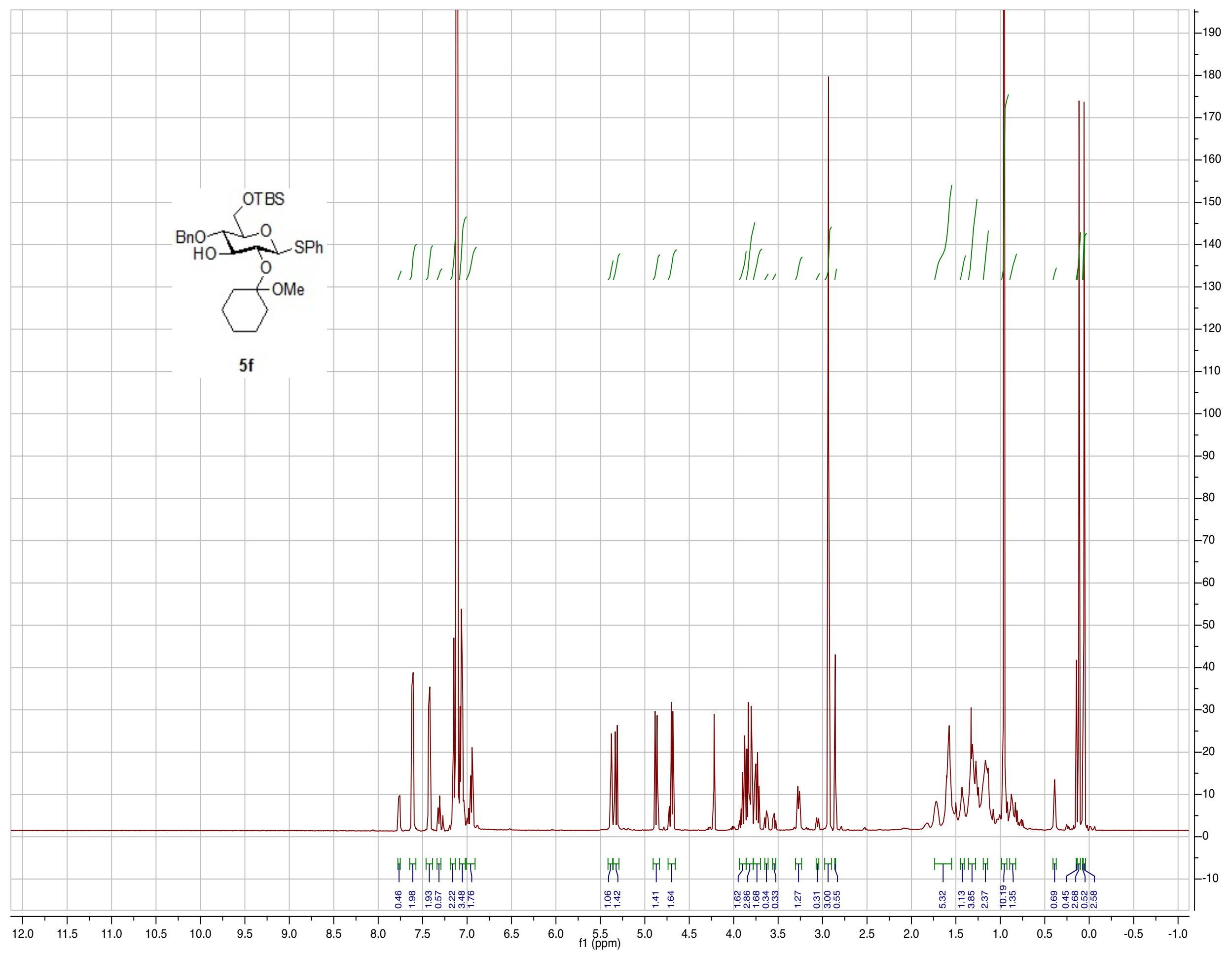




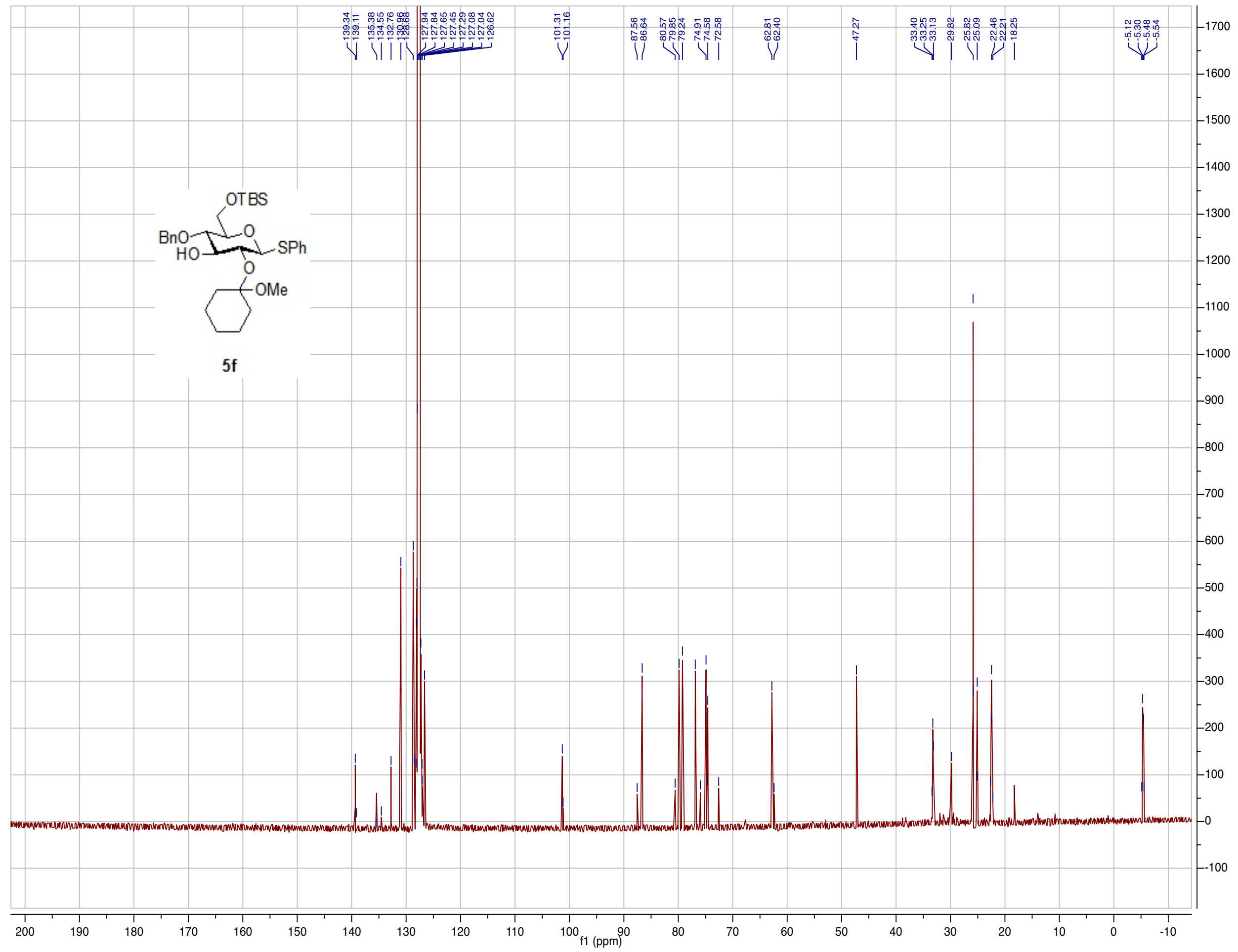




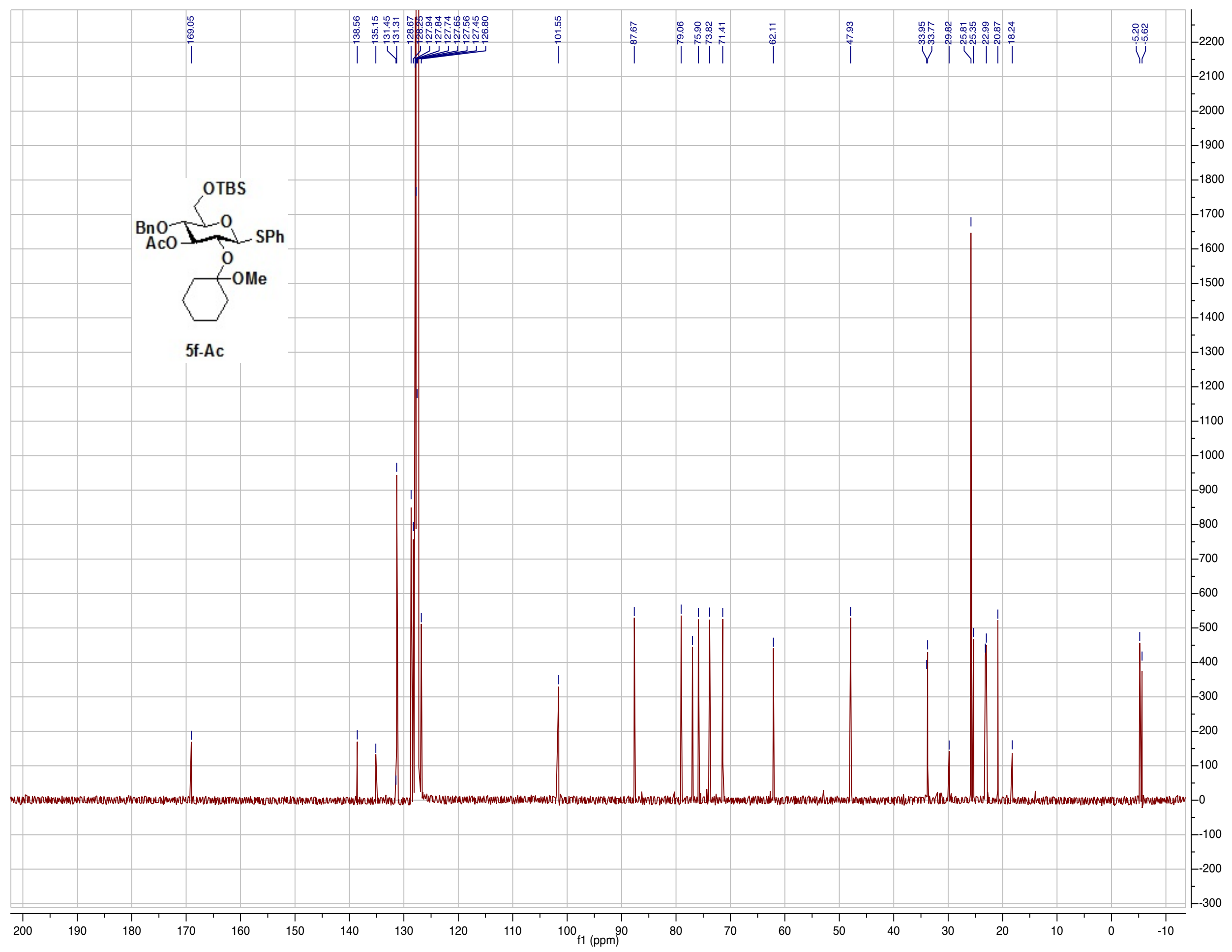




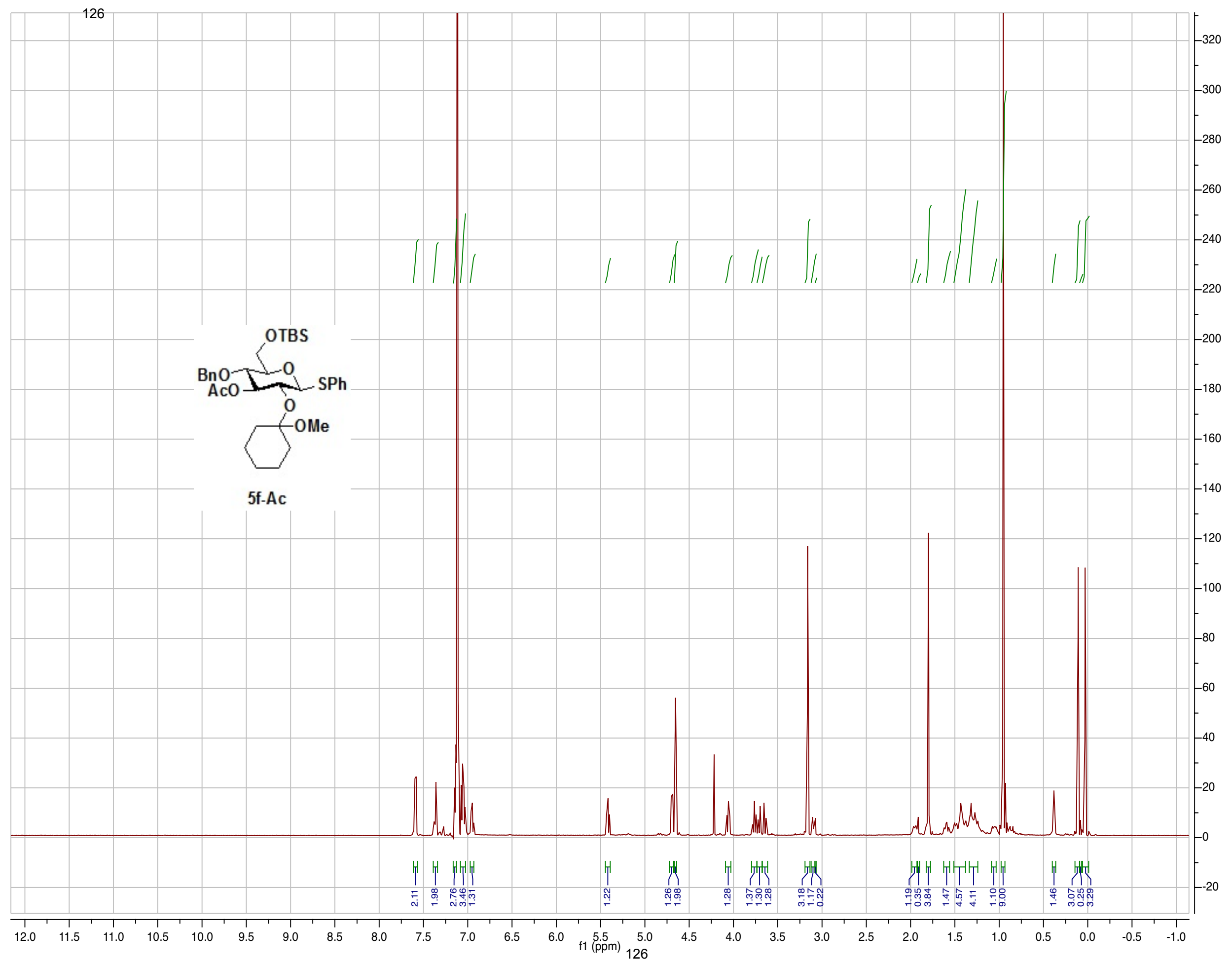


STANDARD PROTON PARAMETERS

Sample Name:

EM-04-268PURECOSY-BENZENE-D6 Data Collected on:

Te-vnmrs 500

Archive directory:

Sample directory

FidFile: EM-04-268COSY-BENZENE-D6

Pulse Sequence: gCosy

Solvent: c6d6

Data collected on: Nov 162012
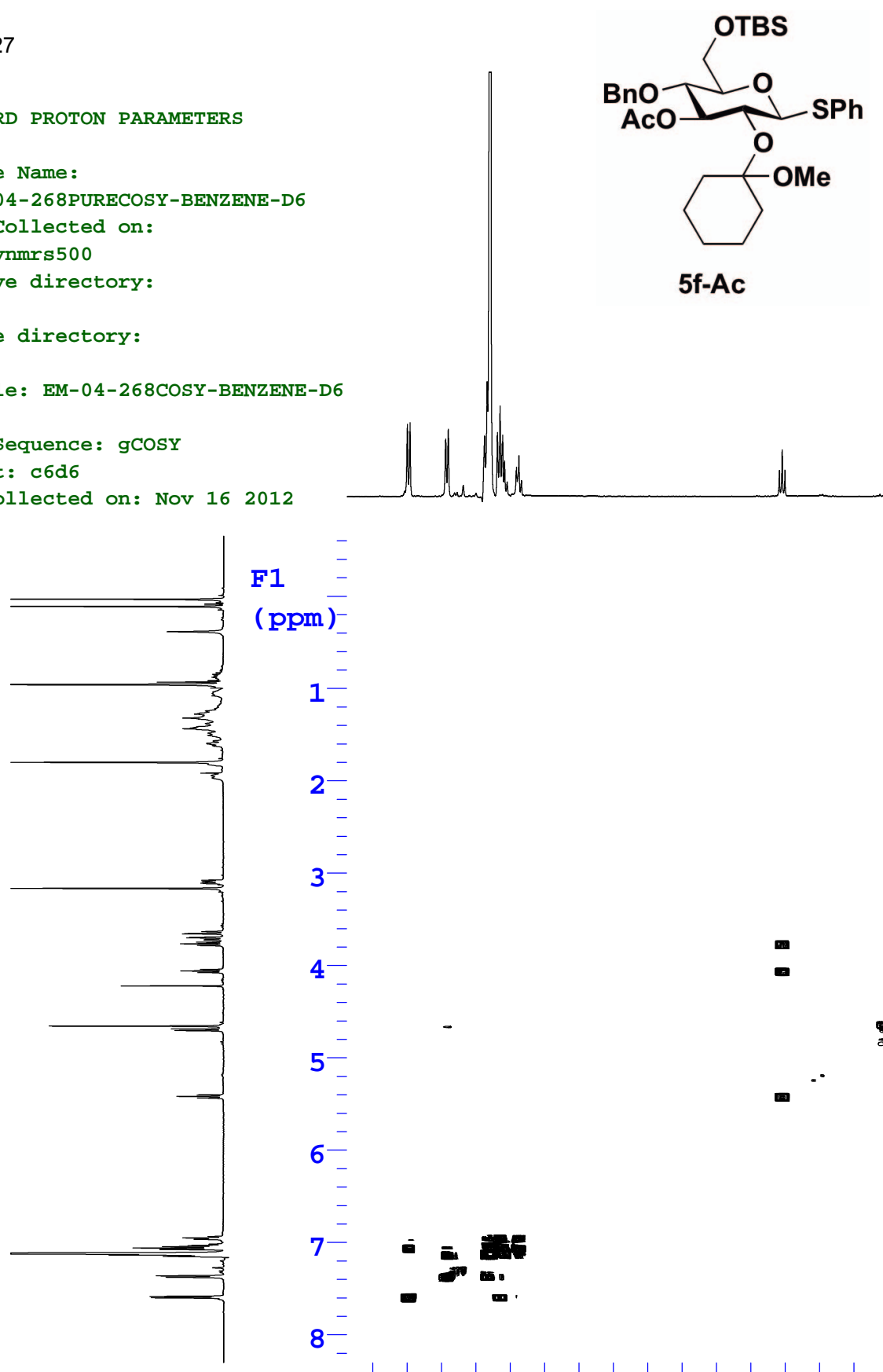

7

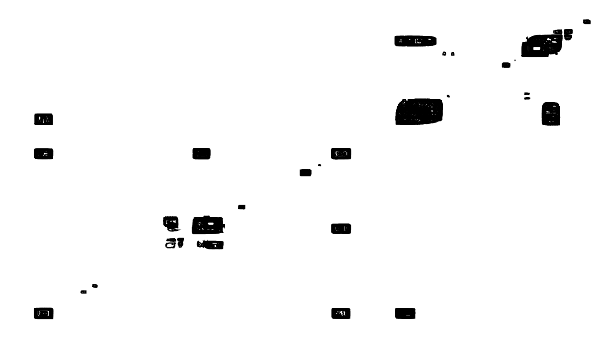




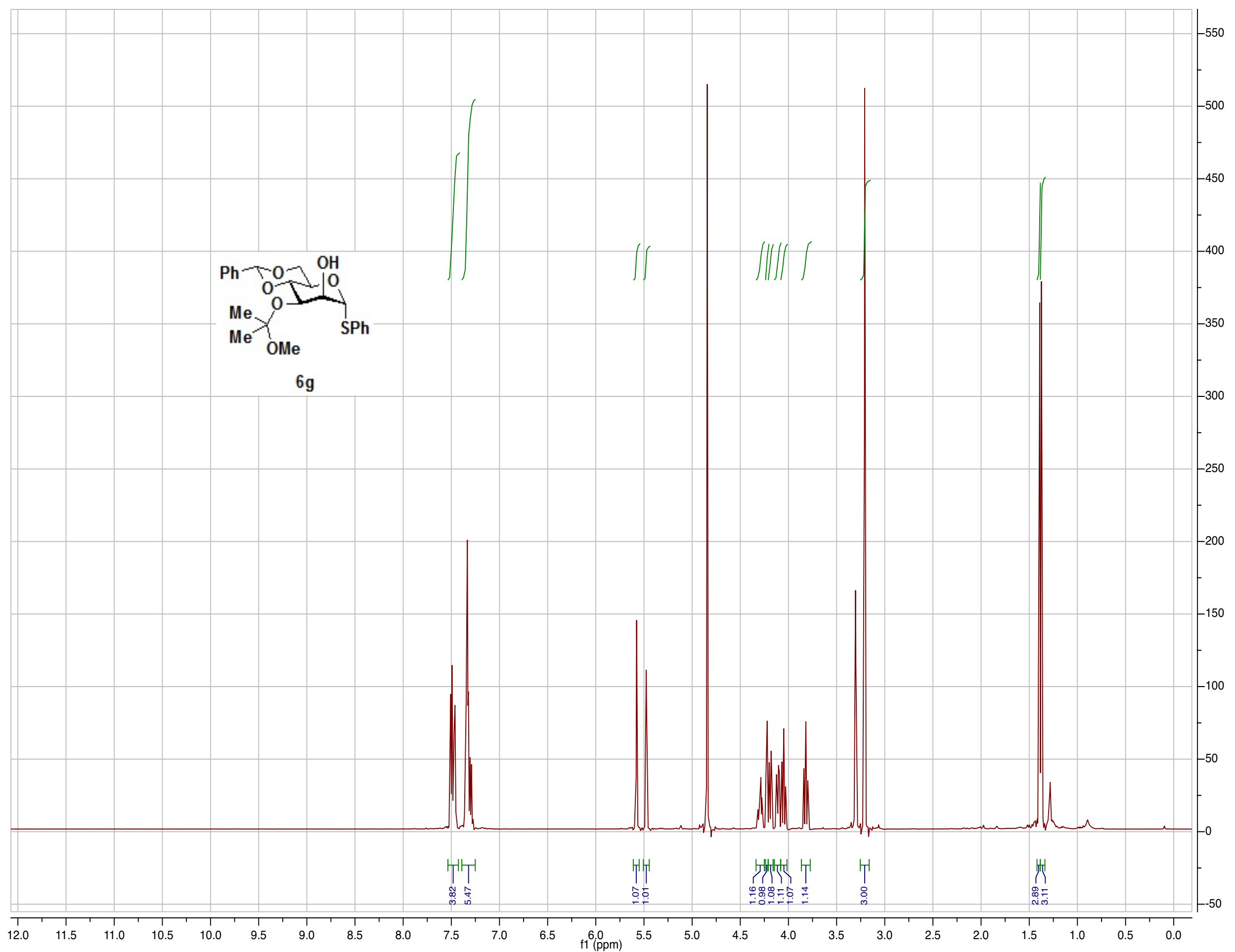




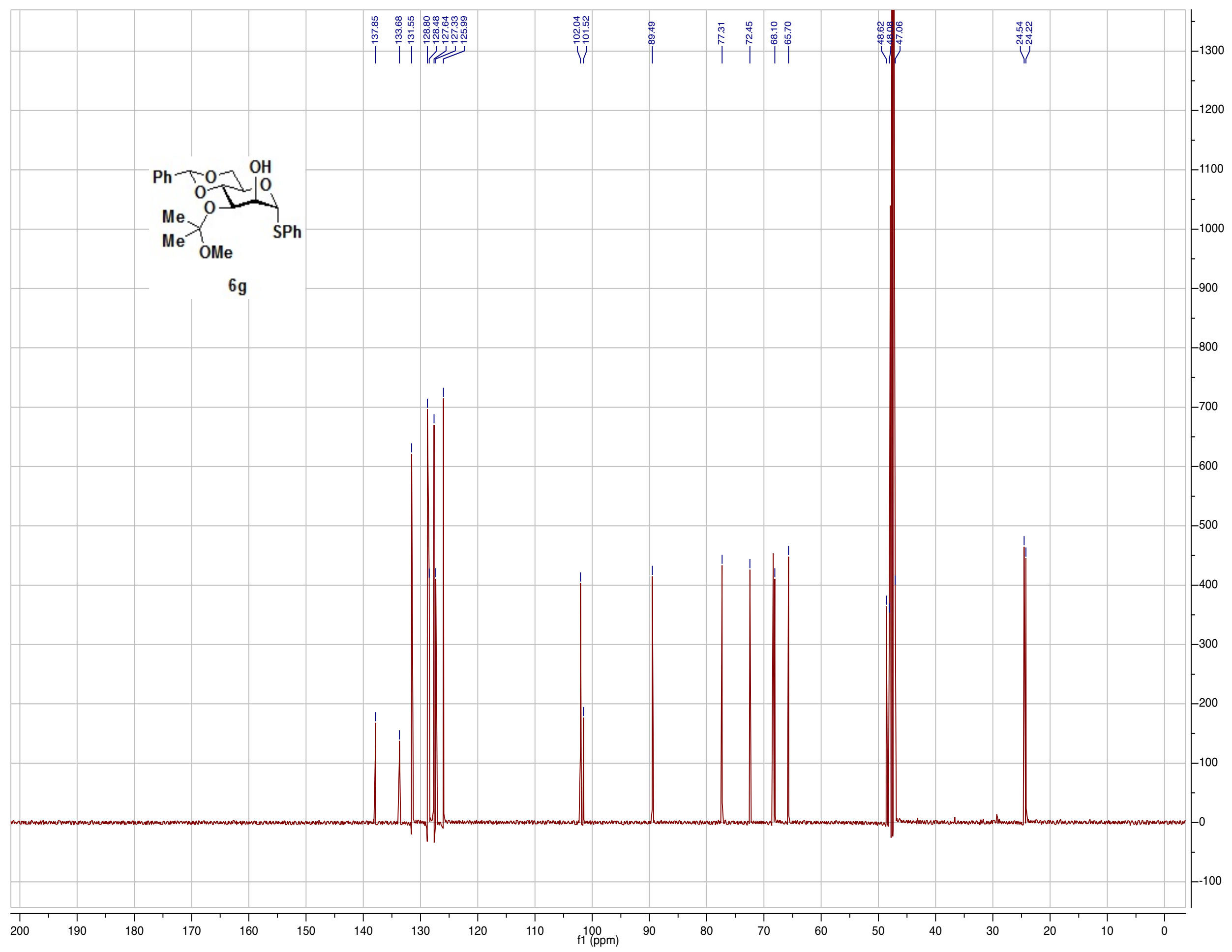




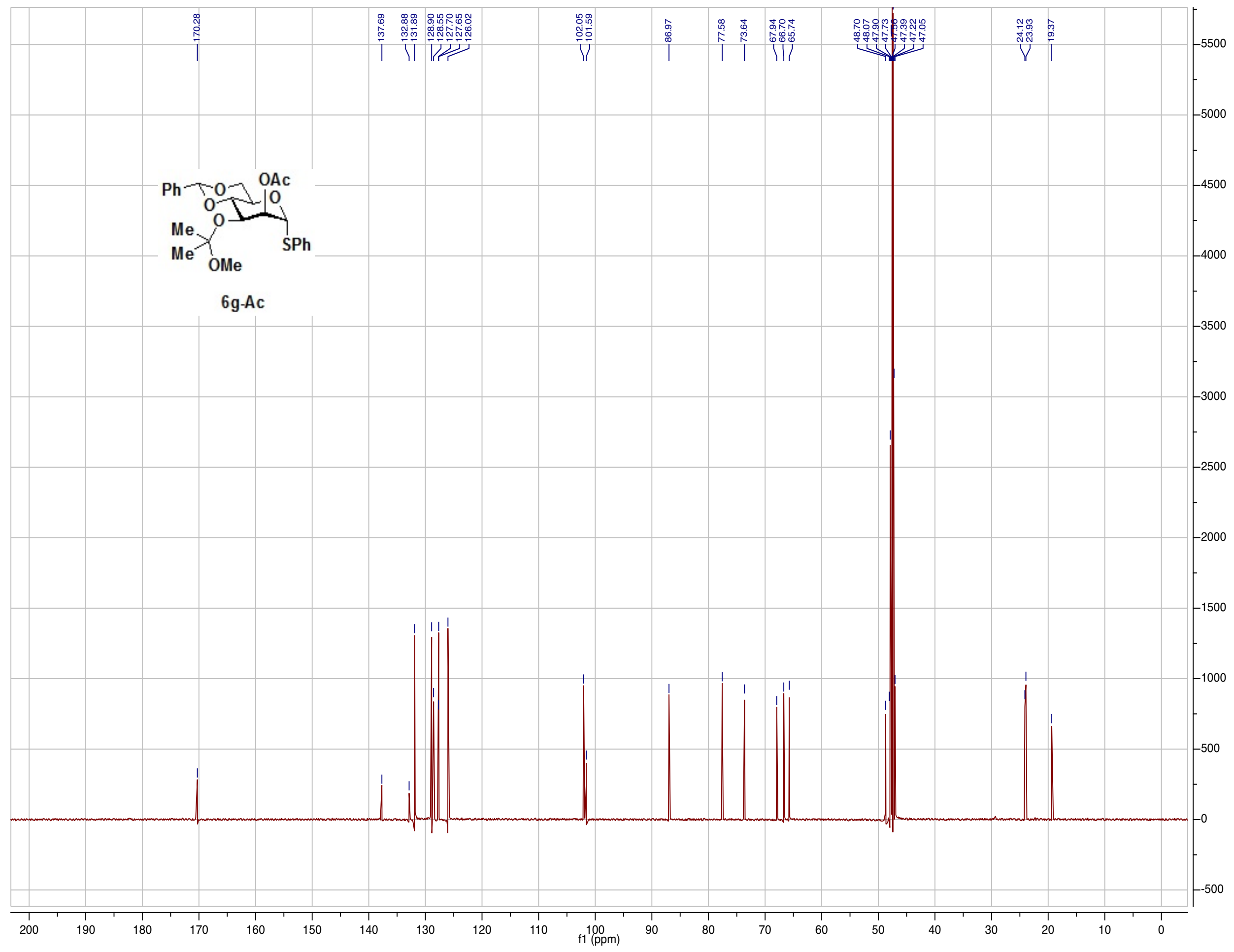




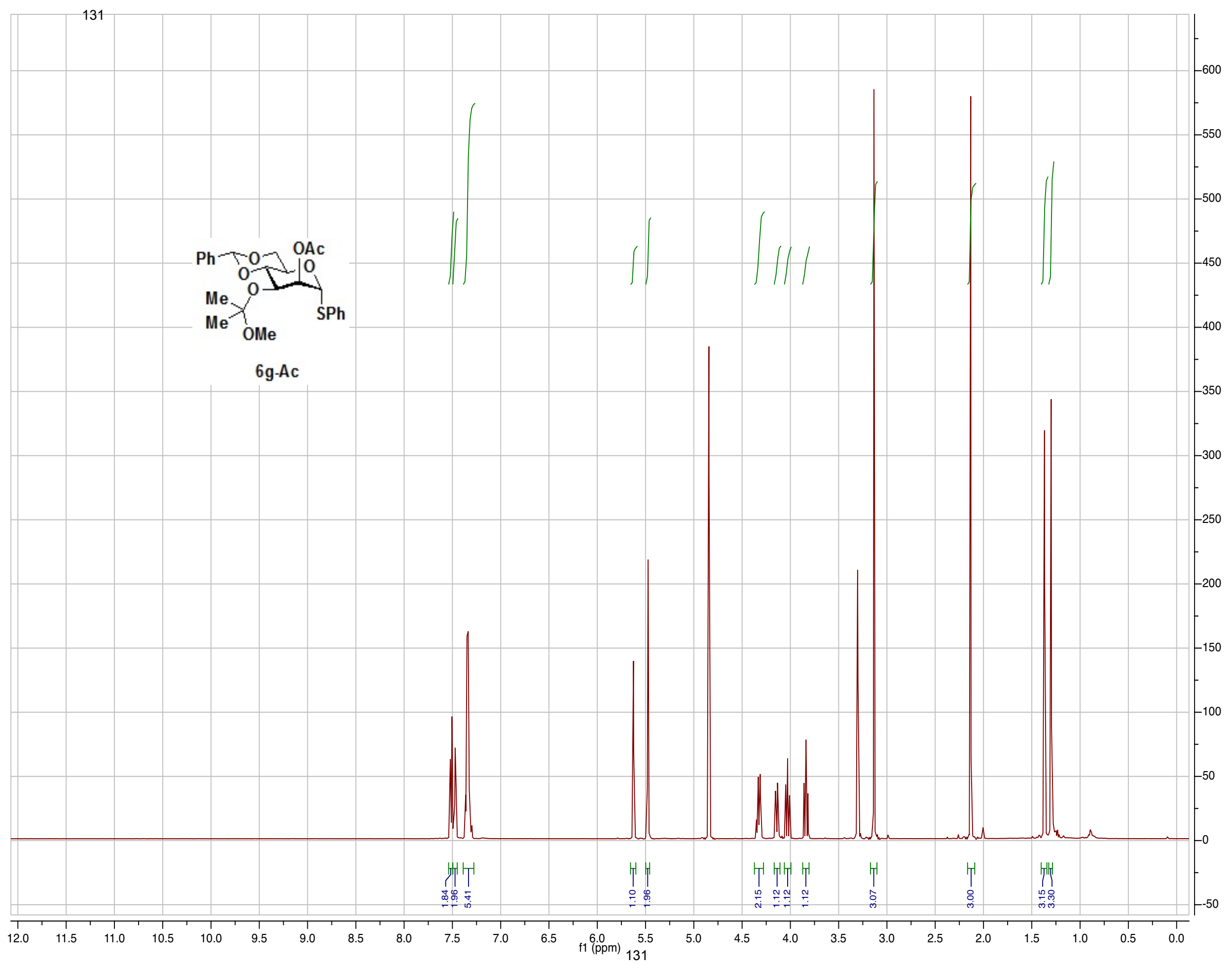


Automated Probe tuning parameter

Sample Name:

EM-05-52PURECOSY-METHANOL-D4

Data Collected on:

Te-vnmrs 500

Archive directory:

Sample directory:

FidFile: EM-05-52PURECOSY-METHANOL-D4

Pulse Sequence: gcosy

Solvent: cd3od

Data collected on: Dec 182012

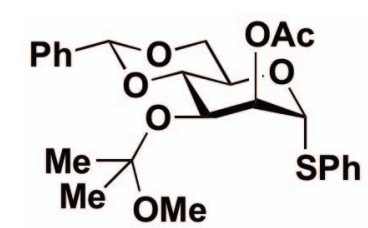

6g-Ac

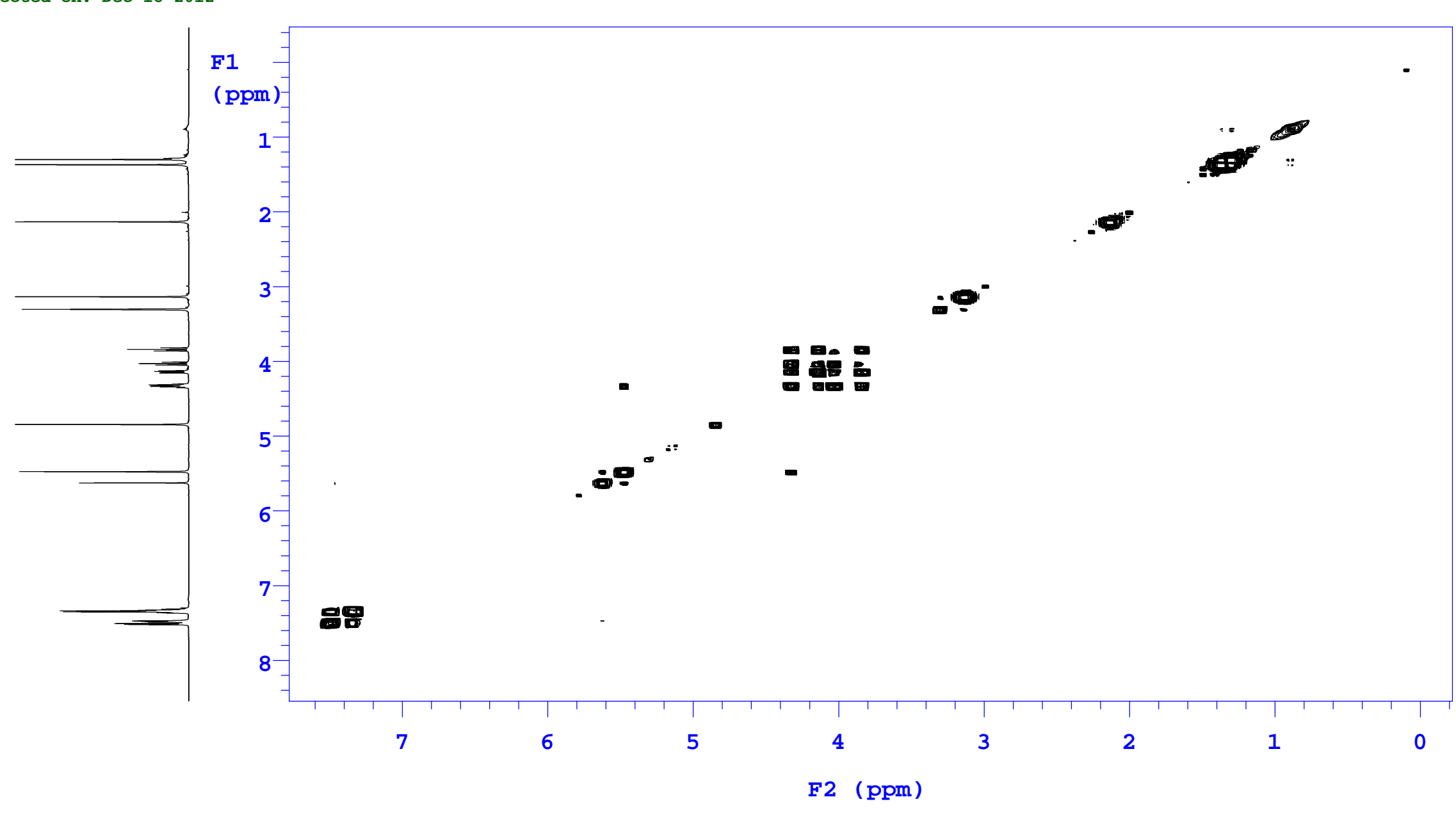




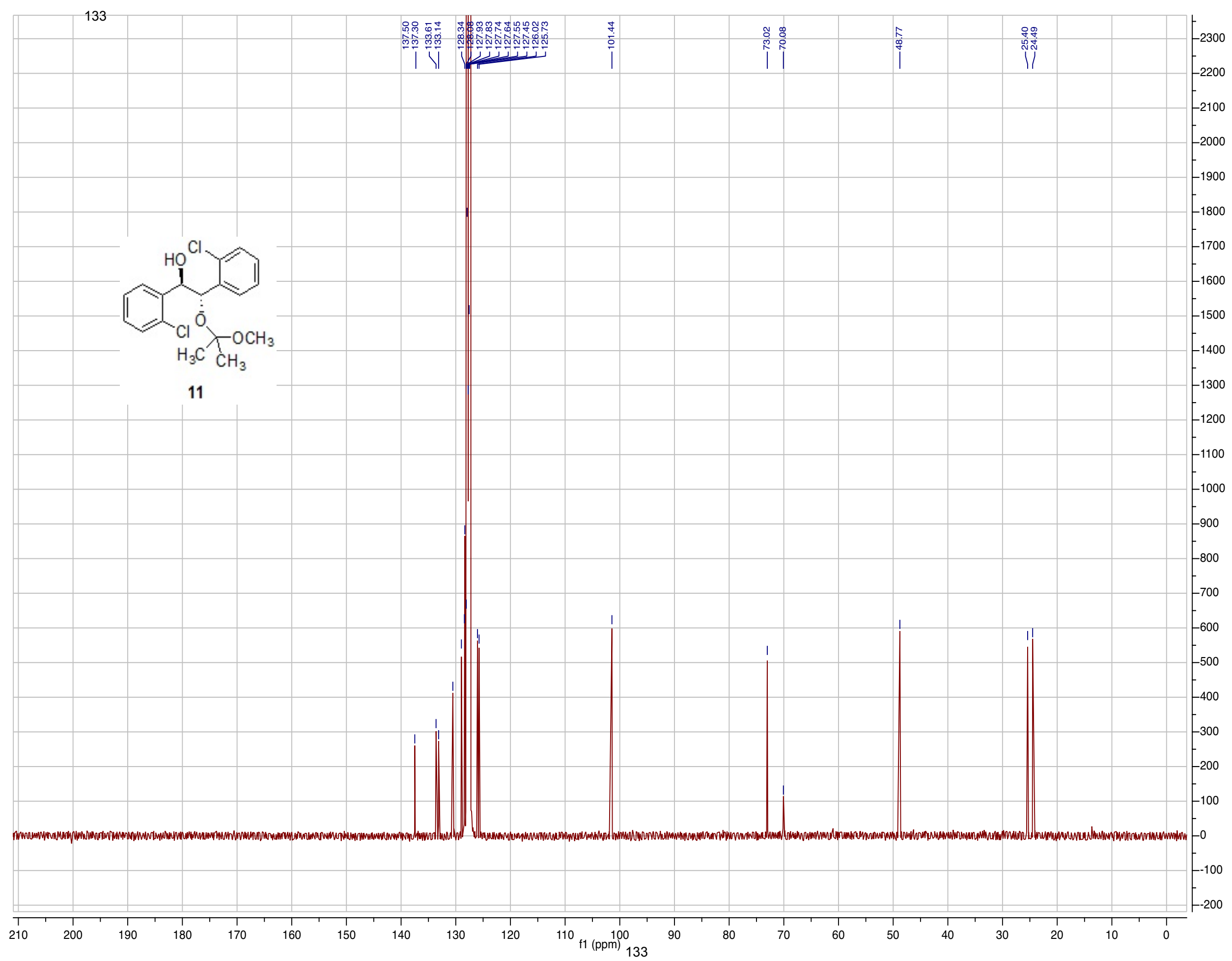




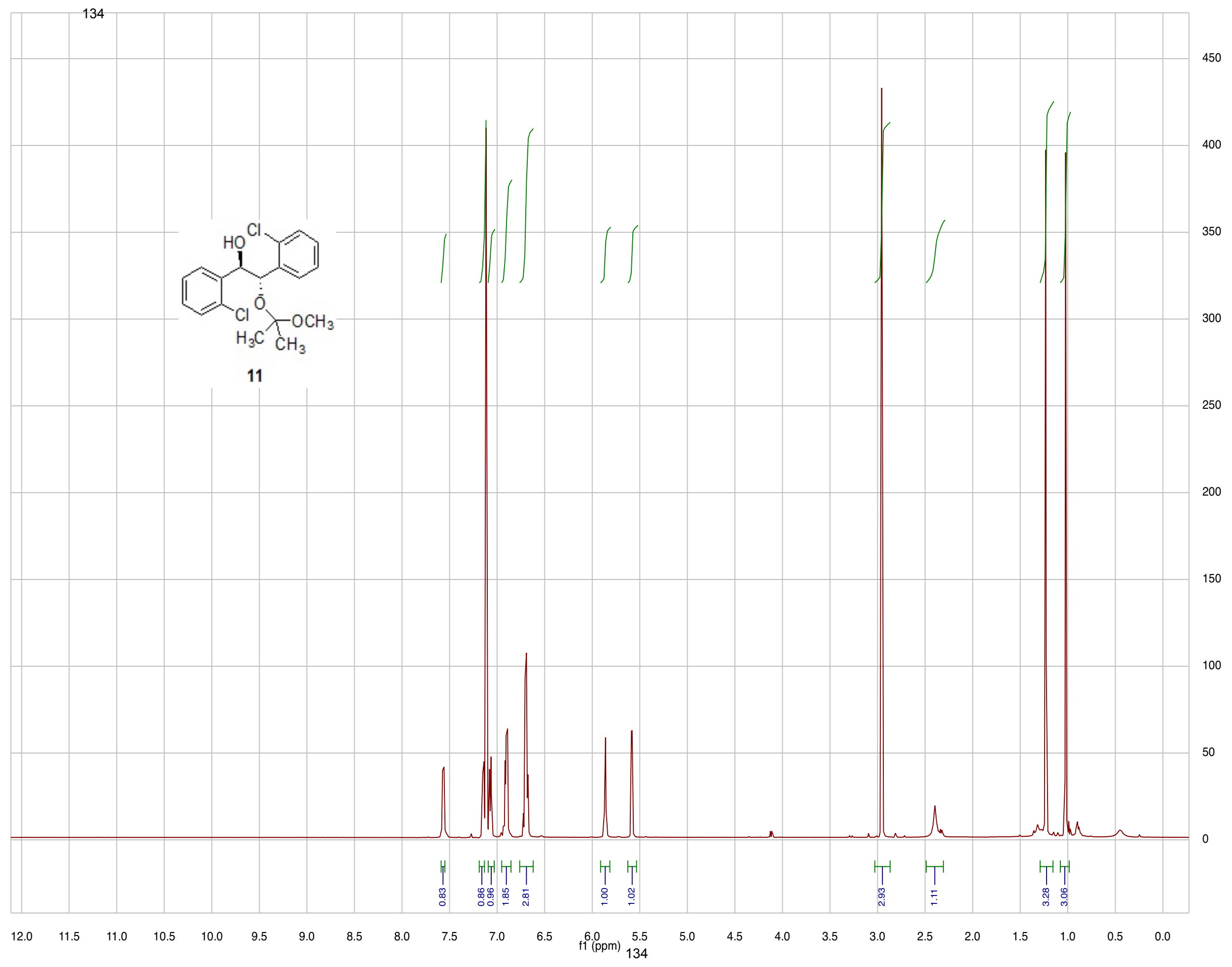




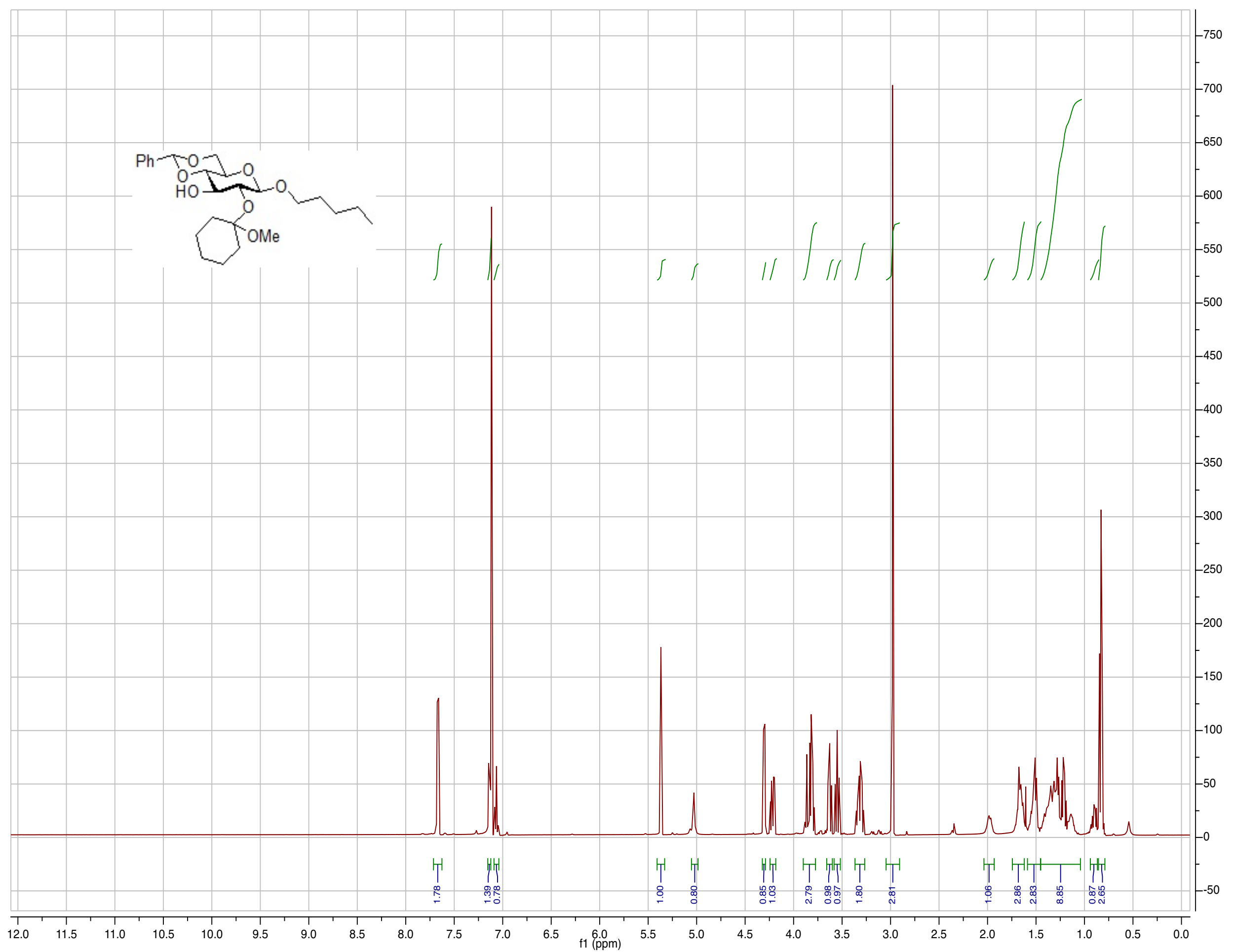




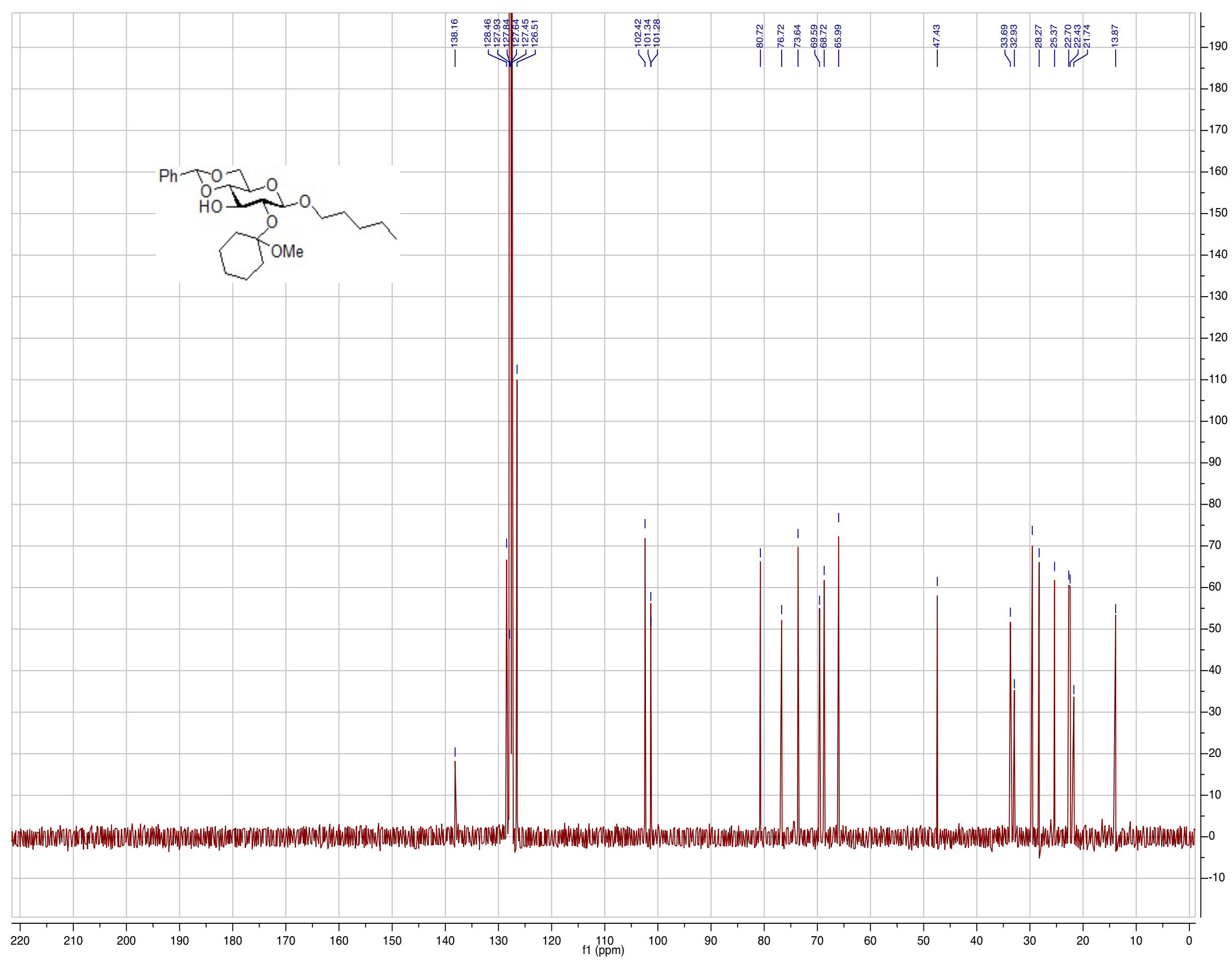




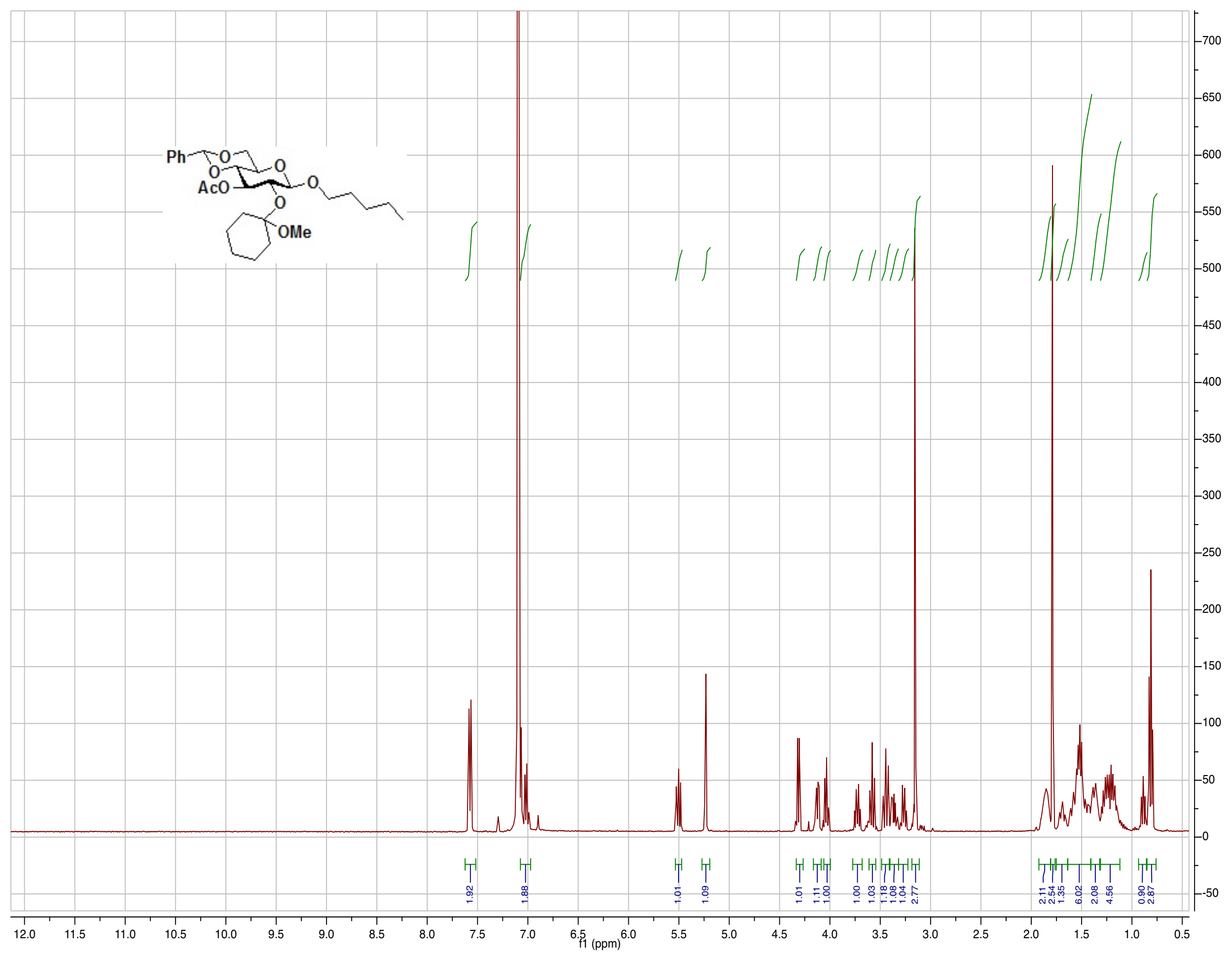




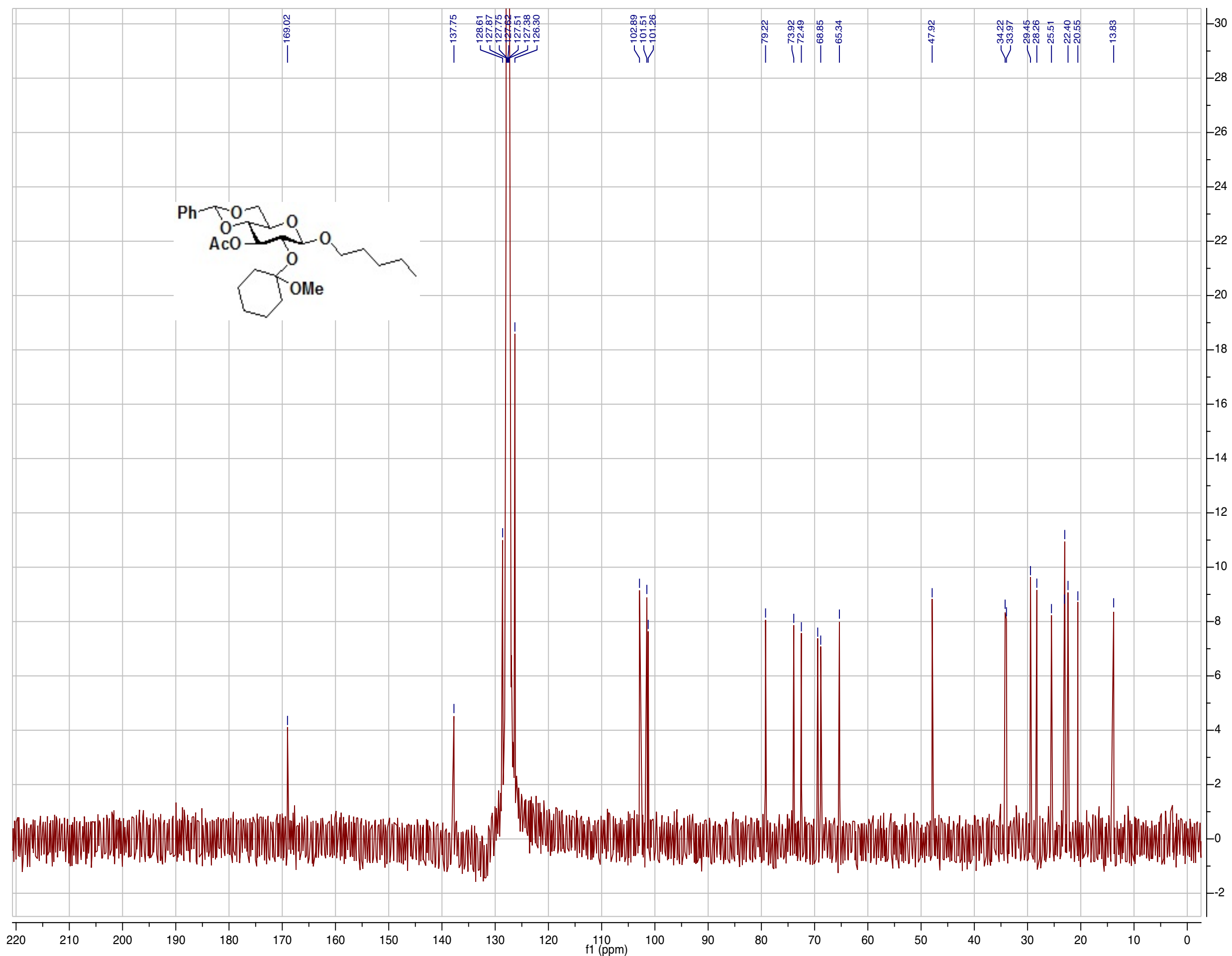




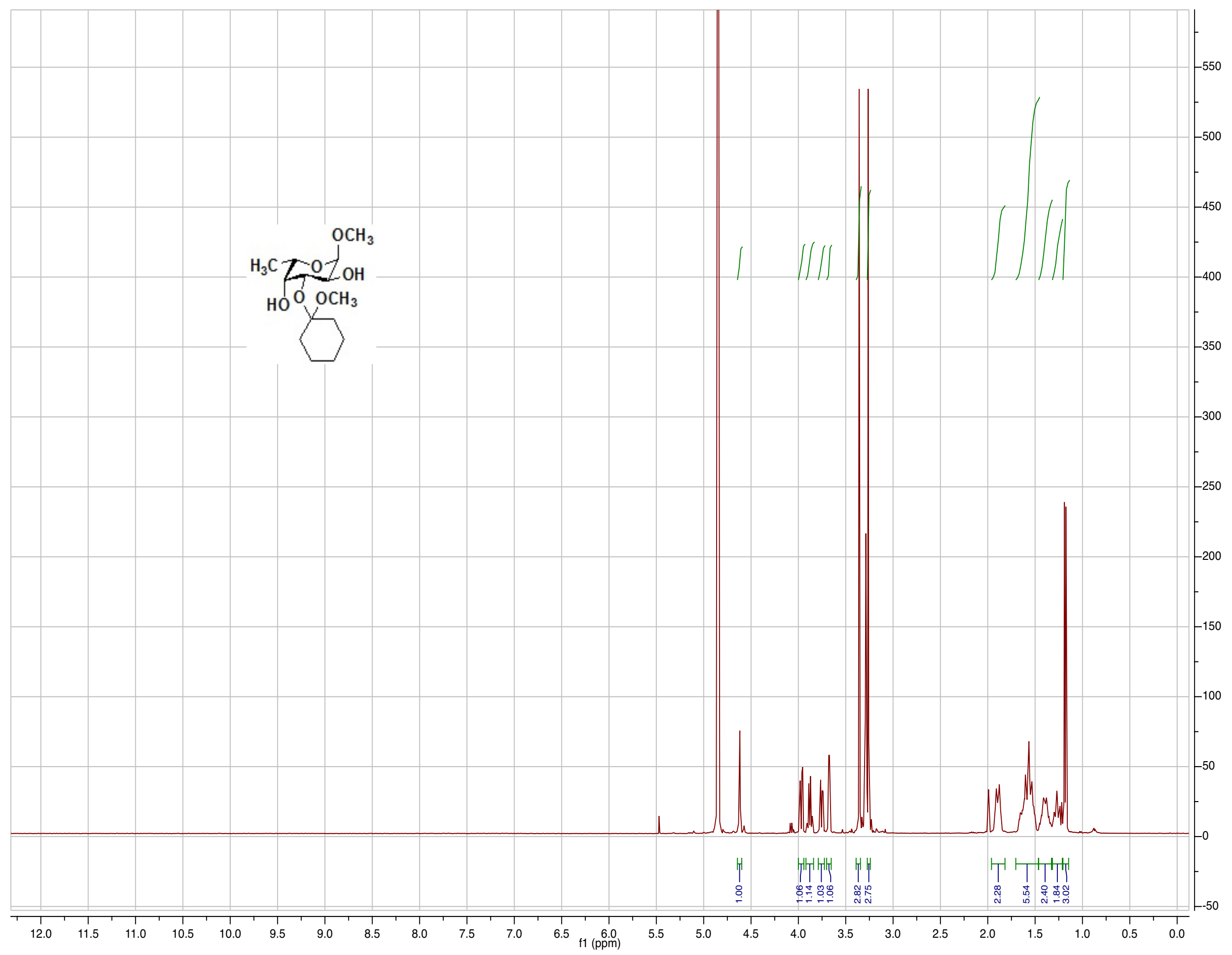




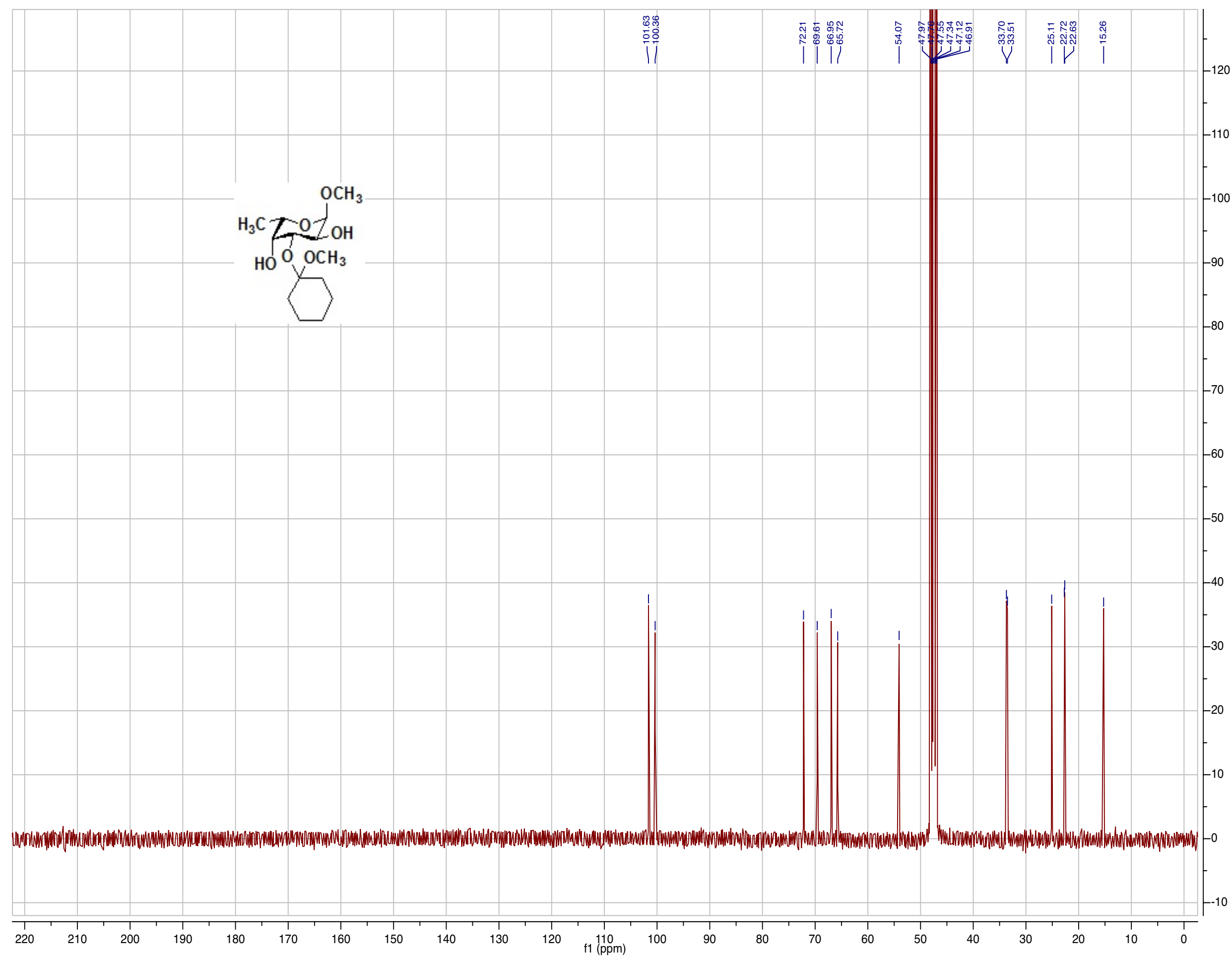




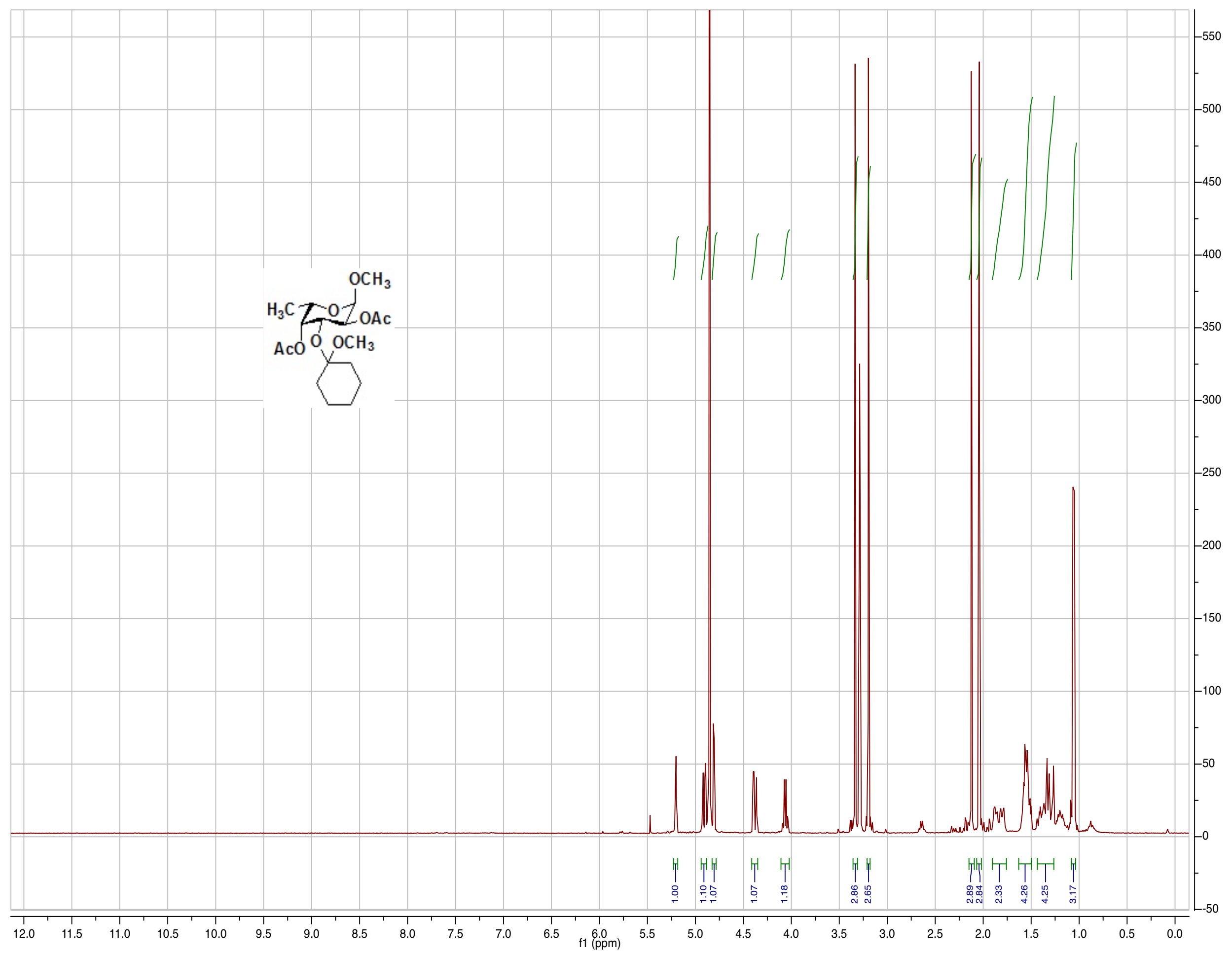




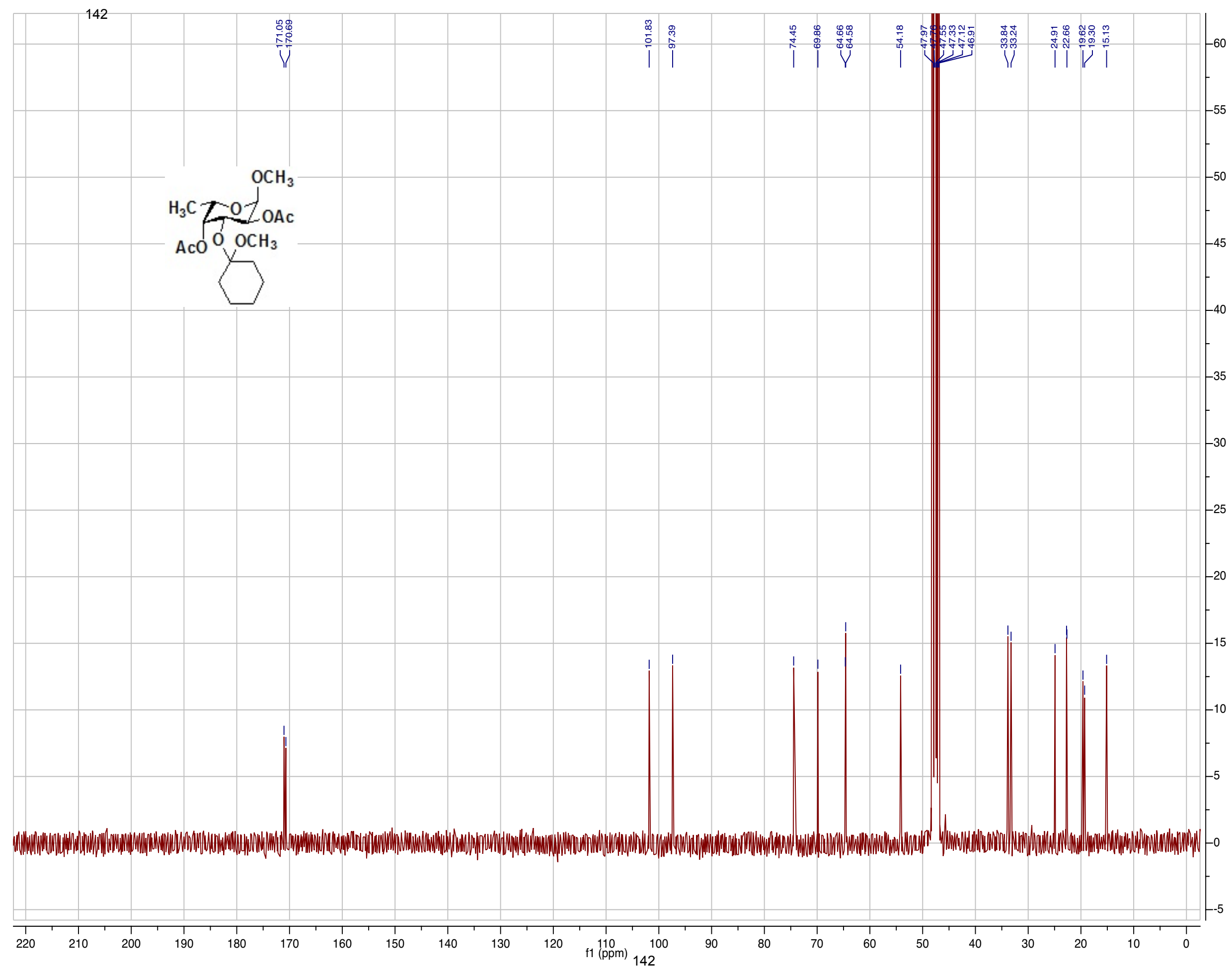




\section{Empower" 3}

\begin{tabular}{|llll|}
\hline & \multicolumn{2}{c|}{ S A M L E IN F O R M A I O N } \\
\hline \hline Sample Name: & EM-05-284CRUDE_99_1_Col & Acquired By: & HPLCuser \\
Sample Type: & Unknown & Sample Set Name & EM05284_99_1_Col 2_40min \\
Vial: & 18 & Acq. Method Set: & 99_1_Col2_40min \\
Injection \#: & 1 & Processing Method & pn_Standard \\
Injection Volume: & $10.00 \mathrm{ul}$ & Channel Name: & 210.2nm@5 \\
Run Time: & 40.0 Minutes & Proc. Chnl. Descr.: & PDA 210.2 nm \\
& & & \\
Date Acquired: & $5 / 14 / 2013$ 8:11:18 PM EDT & & \\
Date Processed: & $5 / 17 / 201311: 47: 25$ AM EDT & & \\
\hline
\end{tabular}

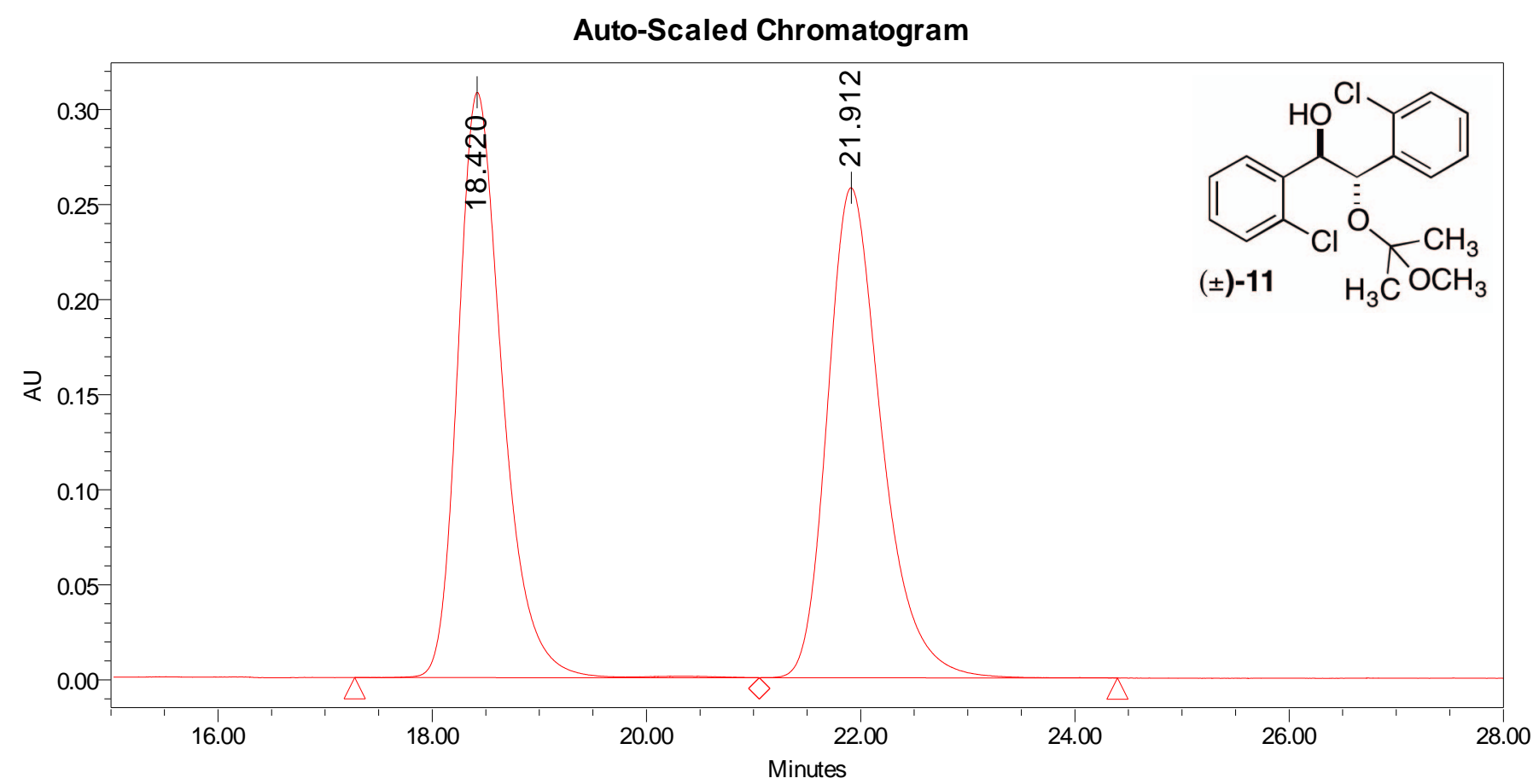

Peak Results

\begin{tabular}{|c|c|c|c|c|}
\hline & Name & RT & Area & Height \\
\hline 1 & & 18.420 & 8884279 & 307862 \\
\hline 2 & & 21.912 & 9036257 & 257709 \\
\hline
\end{tabular}

PDA Result Table

\begin{tabular}{|r|c|c|r|l|}
\hline & Name & RT & $\begin{array}{c}\text { Purity1 } \\
\text { Angle }\end{array}$ & $\begin{array}{c}\text { Purity1 } \\
\text { Threshold }\end{array}$ \\
\hline 1 & & 18.420 & 0.386 & \\
\hline 2 & & 21.912 & 0.402 & \\
\hline
\end{tabular}

Reported by User: HPLCuser

Report Method: 1

Report Method I[ 2072

Page: 1 of 1
Project Name: Enoch Date Printed:

5/17/2013

11:48:35 AM US/Easterr 


\section{Empower" 3}

\begin{tabular}{|llll|}
\hline \multicolumn{2}{c|}{ S A M P E } & IN F O R M A T I O N \\
\hline \hline Sample Name: & EM-05-286CRUDE_99_1_Col & Acquired By: & HPLCuser \\
Sample Type: & Unknown & Sample Set Name & EM05286_99_1_Col 2_40min \\
Vial: & 23 & Acq. Method Set: & 99_1_Col2_40min \\
Injection \#: & 1 & Processing Method & pn_Standard \\
Injection Volume: & $10.00 \mathrm{ul}$ & Channel Name: & $210.2 \mathrm{~nm} @ 5$ \\
Run Time: & 40.0 Minutes & Proc. Chnl. Descr.: & PDA 210.2 nm \\
& & & \\
Date Acquired: & $5 / 15 / 201312: 59: 33$ PM EDT & & \\
Date Processed: & $5 / 17 / 201311: 42: 44$ AM EDT & & \\
\hline
\end{tabular}

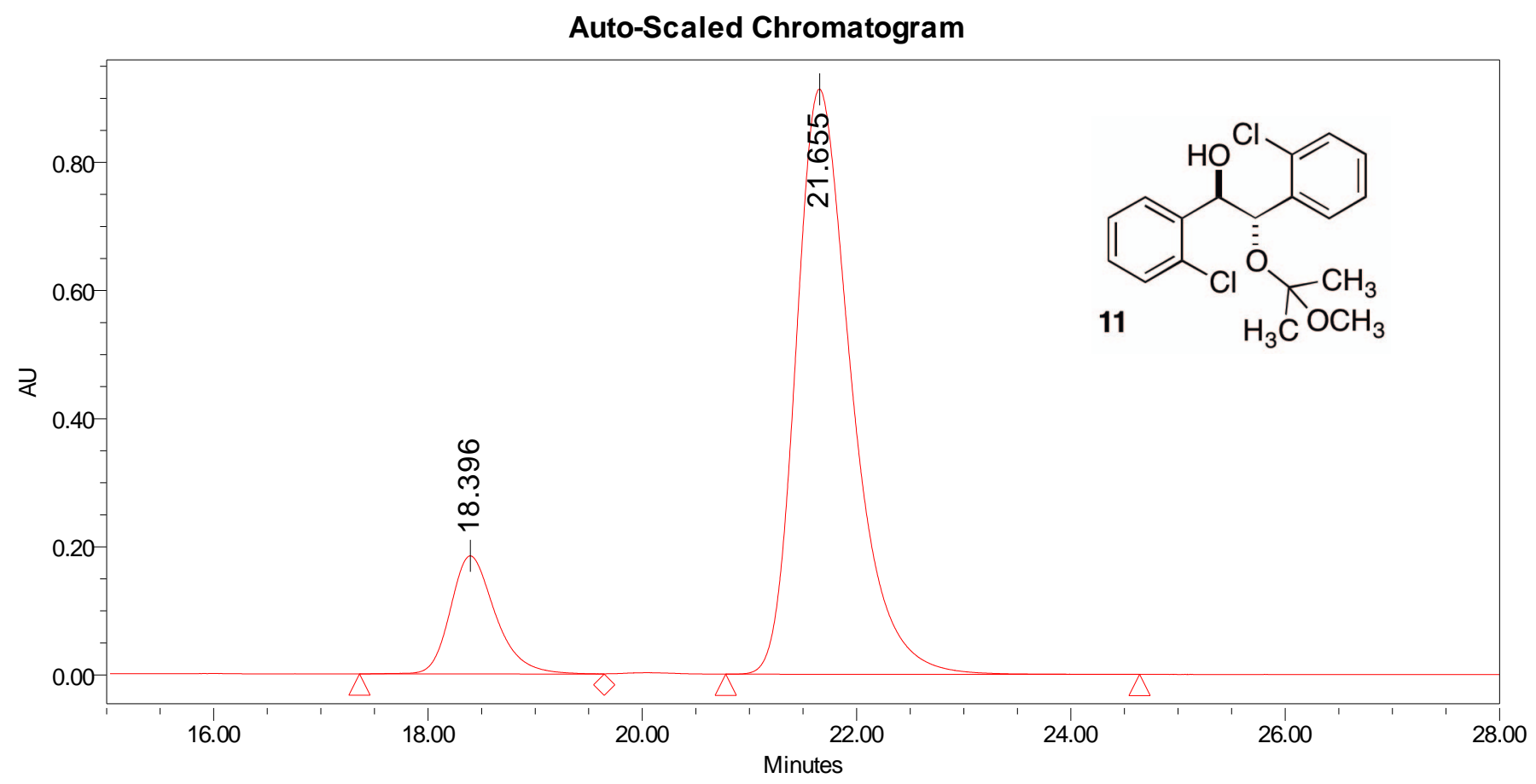

Peak Results

\begin{tabular}{|c|c|c|c|c|}
\hline & Name & RT & Area & Height \\
\hline 1 & & 18.396 & 5389910 & 184313 \\
\hline 2 & & 21.655 & 32834196 & 913054 \\
\hline
\end{tabular}

PDA Result Table

\begin{tabular}{|r|c|c|r|r|}
\hline & Name & RT & $\begin{array}{c}\text { Purity1 } \\
\text { Angle }\end{array}$ & $\begin{array}{c}\text { Purity1 } \\
\text { Threshold }\end{array}$ \\
\hline 1 & & 18.396 & 0.295 & 0.292 \\
\hline 2 & & 21.655 & 0.639 & 0.276 \\
\hline
\end{tabular}

Reported by User: HPLCuser

Report Method: 1

Report Method I[ 2069

Page: 1 of 2
Project Name: Enoch Date Printed:

5/17/2013

11:46:36 AM US/Easterr 